POEF-SH-30

\title{
Nuclear Criticality Safety Calculational Analysis for Small-Diameter Containers
}

\author{
November 1995 \\ by \\ Maria S. LeTellier \\ David J. Smallwood \\ Jeffrey A. Henkel \\ David E. Smith \\ Battelle \\ Under Contract 400183 \\ LOCKHEED MARTIN UTILITY SERVICES, INC. \\ PORTSMOUTH GASEOUS DIFFUSION PLANT \\ P.O. Box 628 Piketon, Ohio 45661 \\ Under Contract USECHQ-93-C-0001 \\ to the \\ U.S. ENRICHMENT CORPORATION
}




\section{DISCLAIMER}

Portions of this document may be illegible in electronic image products. Images are produced from the best available original document. 


\section{NOTICE}

This report was prepared as an account of work sponsored by an agency of the United States Government. Neither the United States Government nor any agency thereof, nor any of their employees makes any warranty. express or implied, or assumes any legal liability or responsibility for the accuracy, completeness, or usefulness of any information, apparatus, product, or process disclosed, or represents that its use would not infringe privately owned rights. Reference herein to any specific commercial product, process, or service by trade name, trademark, manufacturer, or otherwise does not necessarily constitute or imply its endorsement, recommendation, or favoring by the United States Government or any agency thereof. The views and opinions of authors expressed herein do not necessarily state or reflect those of the United States Government or any agency thereof.

Available to DOE and DOE Contractors from the Office of Scientific and Technical Information, P.0. Box 62, Oak Ridge, TN 37831; prices available from (615) 576-8401, FTS/626-8401.

Available to the public from the National Technical Information Service, U.S. Department of Commerce, 5285 Port Royal Road, Springfield, VA 22161. 
POEF-SH-30

\title{
DISTRIBUTION
}

\section{LOCKHEED MARTIN UTILITY SERVICES, INC.}

\author{
PORTSMOUTH
}

R. Oxenham

R. Lemming

D. D'Aquila (10)
Central Files (2)

X-710 Technical Library (2)

Technical Review (2)
R. Tayloe
D. Smallwood
J. Henkel
D. Smith
B. Lee
V. Pasupathi
M. LeTellier
D. Lindenschmidt
C. Haught
H. Dyer
C. Jordan
S. Love
K. Shaffer 


\section{TABLE OF CONTENTS}

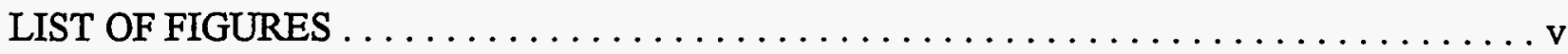

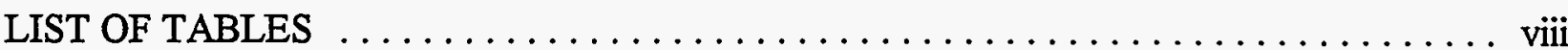

1.0 INTRODUCTION $\ldots \ldots \ldots \ldots \ldots \ldots \ldots \ldots \ldots \ldots \ldots \ldots \ldots \ldots \ldots \ldots \ldots \ldots \ldots \ldots$

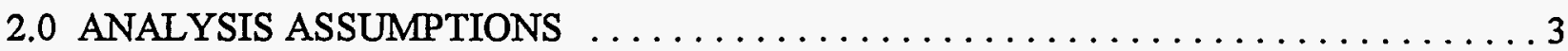

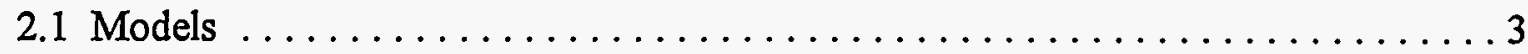

2.1.1 Description of Standard Geometry Models $\ldots \ldots \ldots \ldots \ldots \ldots \ldots 4$

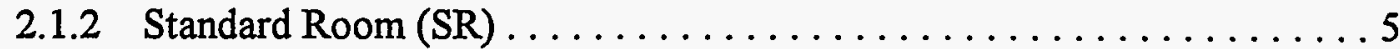

2.1.3 Single Container Standard Reflection Models $\ldots \ldots \ldots \ldots \ldots \ldots 5$

2.1.4 Two Containers Touching Reflection Models $\ldots \ldots \ldots \ldots \ldots \ldots 7$

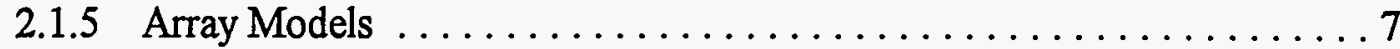

2.2 Uranium Compounds Analyzed $\ldots \ldots \ldots \ldots \ldots \ldots \ldots \ldots \ldots \ldots \ldots \ldots \ldots$

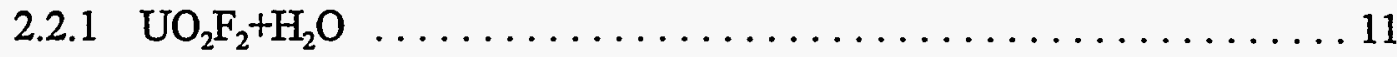

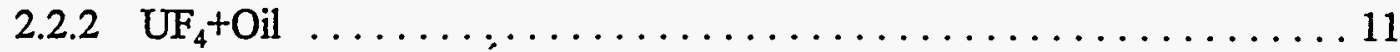

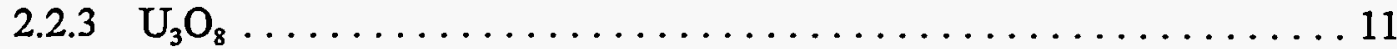

3.0 DETERMINATION OF NUMBER DENSITIES $\ldots \ldots \ldots \ldots \ldots \ldots \ldots \ldots \ldots \ldots$

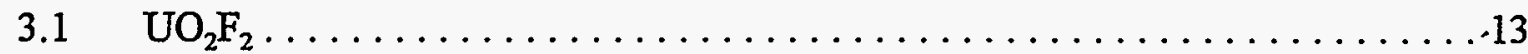

3.1.1 Determination of Number Density . ................... 13

3.1.2 Justification for Using a Uranium Density of $3.2 \mathrm{~g} / \mathrm{cc}$ for $\mathrm{H} / \mathrm{U}$ Less

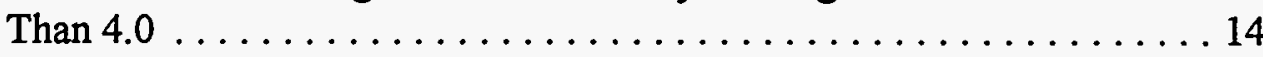

$3.2 \mathrm{UF}_{4}+$ Oil Number Density Determination $\ldots \ldots \ldots \ldots \ldots \ldots \ldots \ldots \ldots$

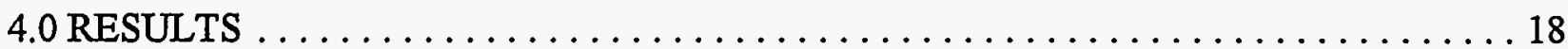

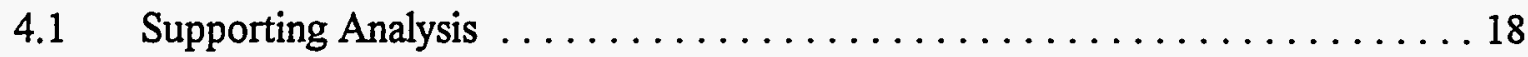

4.1.1 Use of Barber Formula for $\mathrm{H} / \mathrm{U}$ Less Than $4.0 \ldots \ldots \ldots \ldots \ldots$

4.1.2 Container Thickness Study ........................ 19

4.1.3 Random Number Generation ..................... 20

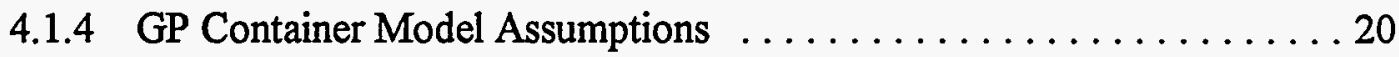

4.2 General Discussion of Plotted $\mathrm{k}_{\mathrm{eff}}$ versus $\mathrm{H} / \mathrm{U}$ Results $\ldots \ldots \ldots \ldots \ldots \ldots 25$

$4.3 \quad 4.32$-inch-Diameter by 50 -inch Polyethylene Bottle $\ldots \ldots \ldots \ldots \ldots \ldots 25$

$4.3 .1 \quad 100 \%$ Enriched $\mathrm{UO}_{2} \mathrm{~F}_{2}+\mathrm{H}_{2} \mathrm{O}$ and $\mathrm{UF}_{4}+\mathrm{Oil} \ldots \ldots \ldots \ldots \ldots \ldots \ldots \ldots \ldots \ldots \ldots \ldots \ldots \ldots$

4.3.2 $10 \%$ Enriched $\mathrm{UO}_{2} \mathrm{~F}_{2}+\mathrm{H}_{2} \mathrm{O}$ and $\mathrm{UF}_{4}+\mathrm{Oil} \ldots \ldots \ldots \ldots \ldots \ldots \ldots$

4.45 .0 -inch-Diameter by 24 -inch-Tall Polyethylene Container $\ldots \ldots \ldots \ldots \ldots 52$

4.4.1 $100 \%$ Enriched $\mathrm{UO}_{2} \mathrm{~F}_{2}+\mathrm{H}_{2} \mathrm{O}$ and $\mathrm{UF}_{4}+\mathrm{Oil} \ldots \ldots \ldots \ldots \ldots \ldots \ldots \ldots \ldots \ldots \ldots \ldots$

4.4.2 $10 \%$ Enriched $\mathrm{UO}_{2} \mathrm{~F}_{2}+\mathrm{H}_{2} \mathrm{O}$ and $\mathrm{UF}_{4}+\mathrm{Oil} \ldots \ldots \ldots \ldots \ldots \ldots \ldots \ldots \ldots \ldots \ldots \ldots$

4.4.3 5.0-inch-Diameter Case $\left(\mathrm{UF}_{4}+\mathrm{Oil}\right) \ldots \ldots \ldots \ldots \ldots \ldots \ldots \ldots \ldots \ldots \ldots \ldots \ldots$ 
POEF-SH-30

4.5 5.25-inch-Diameter by 24-inch-Tall Tin-Plated Steel Container . . . . . . . 76

$4.5 .1100 \%$ Enriched $\mathrm{UO}_{2} \mathrm{~F}_{2}+\mathrm{H}_{2} \mathrm{O}$ and $\mathrm{UF}_{4}+\mathrm{Oil} \ldots \ldots \ldots \ldots \ldots \ldots$

$4.5 .210 \%$ Enriched $\mathrm{UO}_{2} \mathrm{~F}_{2}+\mathrm{H}_{2} \mathrm{O}$ and $\mathrm{UF}_{4}+\mathrm{Oil} \ldots \ldots \ldots \ldots$

$4.6 \quad 5.25$-inch-Diameter by 15 -inch Tin Can $\ldots \ldots \ldots \ldots \ldots \ldots \ldots \ldots \ldots$

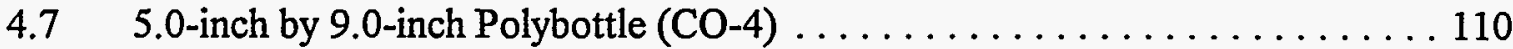

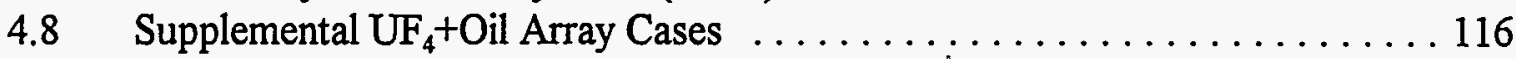

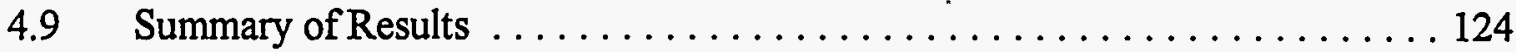

5.0 REFERENCES ............................... 127

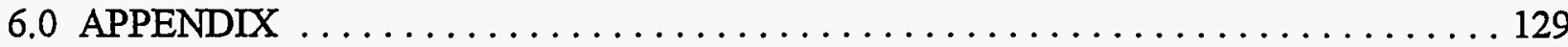




\section{LIST OF FIGURES}

Figure 2.1.3-1. Single Container in a Corner with Water Reflector Simulating a Person . . . . 6

Figure 2.1.4-1. Two Containers Next to a Wall with Water Reflector Simulating a Person . . 8

Figure 2.1.4-2. Two Containers 1-Foot from a Wall with Water Reflector Simulating

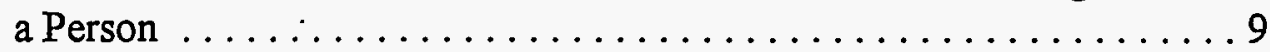

Figure 2.1.5-1. Standard Array Configuration with Concrete Reflector ............ 10

Figure 4.1.2-1. Thickness Study for a Single Container of $\mathrm{UO}_{2} \mathrm{~F}_{2} \ldots \ldots \ldots \ldots \ldots \ldots 21$

Figure 4.1.2-2. Thickness Study for Two 5.00" ID x 24" Tall Cylinders of $\mathrm{UO}_{2} \mathrm{~F}_{2} \ldots \ldots \ldots 22$

Figure 4.1.2-3. Thickness Study for a Single Container of $\mathrm{UF}_{4}$ and $\mathrm{Oil} \ldots \ldots \ldots \ldots \ldots 23$

Figure 4.1.2-4. Thickness Study for Two 5.00" $\mathrm{DD}$ x 24" Tall Cylinders of $\mathrm{UF}_{4}$ and Oil . . . 24

Figure 4.3.1-1. Single Fully Reflected 4.32" ID x 50" Tall Cylinder of $\mathrm{UO}_{2} \mathrm{~F}_{2} \ldots \ldots \ldots \ldots 27$

Figure 4.3.1-2. Single 4.32" $\mathrm{ID} \times 50^{\prime \prime}$ Tall Cylinder of $\mathrm{UO}_{2} \mathrm{~F}_{2}$ in a Concrete Corner ...... 28

Figure 4.3.1-3. Two 4.32" $\mathrm{ID} \times 50$ " Tall Cylinders of $\mathrm{UO}_{2} \mathrm{~F}_{2}$ Next to a Wall .........29

Figure 4.3.1-4. Two 4.32" ID x 50" Tall Cylinders of $\mathrm{UO}_{2} \mathrm{~F}_{2}$ One Foot from a Wall ...... 30

Figure 4.3.1-5. Single Fully Reflected 4.32" ID x 50" Tall Cylinder of $\mathrm{UF}_{4}$ and Oil ..... 31

Figure 4.3.1-6. Single 4.32" $\mathrm{ID} \times 50^{\prime \prime}$ Tall Cylinder of $\mathrm{UF}_{4}$ and Oil in a Concrete Corner . . 32

Figure 4.3.1-7. Two 4.32" $\mathrm{ID} \times 50^{\prime \prime}$ Tall Cylinders of $\mathrm{UF}_{4}$ and Oil Next to a Wall ...... 33

Figure 4.3.1-8. Two 4.32" ID x 50" Tall Cylinders of $\mathrm{UF}_{4}$ and Oil One Foot from a Wall . . . 34

Figure 4.3.1-9. -An Array of 4.32" ID x 50" Tall Cylinders of $\mathrm{UO}_{2} \mathrm{~F}_{2} \ldots \ldots \ldots \ldots \ldots$

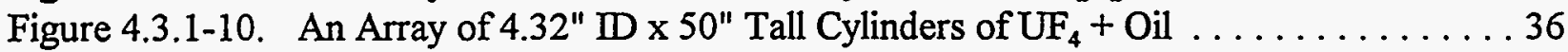

Figure 4.3.1-11. The Effects of Interstitial Water Volume Fraction on $k_{\text {eff }} \ldots \ldots \ldots \ldots . \ldots 37$

Figure 4.3.1-11a. The Effects of Interstitial Water Volume Fraction on $k_{\text {eff }} \ldots \ldots \ldots \ldots . \ldots 38$

Figure 4.3.1-12. The Effects of Interstitial Water Volume Fraction on $k_{\text {eff }} \ldots \ldots \ldots \ldots . \ldots 39$

Figure 4.3.1-12a. The Effects of Interstitial Water Volume Fraction on $k_{\text {eff }} \ldots \ldots \ldots \ldots \ldots 40$

Figure 4.3.1-13. An Array of 4.32" ID x 50" Tall Cylinders of $\mathrm{UO}_{2} \mathrm{~F}_{2} \ldots \ldots \ldots \ldots \ldots 41$

Figure 4.3.1-14. An Array of 4.32" ID x 50" Tall Cylinders of $\mathrm{UF}_{4}+$ Oil . . . . . . . . 42

Figure 4.3.2-1. Single Fully Reflected 4.32" ID x 50" Tall Cylinder of $\mathrm{UO}_{2} \mathrm{~F}_{2}(10 \%) \ldots .44$

Figure 4.3.2-2. Single 4.32" ID x 50" Tall Cylinder of $\mathrm{UO}_{2} \mathrm{~F}_{2}$ in a Concrete Corner (10\%) . 45

Figure 4.3.2-3. Two 4.32" $\mathrm{I} \times$ x 50" Tall Cylinders of $\mathrm{UO}_{2} \mathrm{~F}_{2}$ Next to a Wall $(10 \%) \ldots \ldots 46$

Figure 4.3.2-4. Two 4.32" ID x 50" Tall Cylinders of $\mathrm{UO}_{2} \mathrm{~F}_{2}$ One Foot from a Wall (10\%) . 47

Figure 4.3.2-5. Single Fully Reflected 4.32" ID x 50" Tall Cylinder of $\mathrm{UF}_{4}$ and Oil (10\%) . . 48 .

Figure 4.3.2-6. Single 4.32" ID x 50" Tall Cylinder of $\mathrm{UF}_{4}$ and Oil in a

Concrete Corner $(10 \%) \ldots \ldots \ldots \ldots \ldots \ldots \ldots \ldots . \ldots \ldots$

Figure 4.3.2-7. Two 4.32" $\mathrm{ID} \times 50^{\prime \prime}$ Tall Cylinders of $\mathrm{UF}_{4}$ and Oil Next to a Wall (10\%) . . 50

Figure 4.3.2-8. Two 4.32" $\mathrm{ID} \times$ 50" Tall Cylinders of $\mathrm{UF}_{4}$ and Oil One Foot

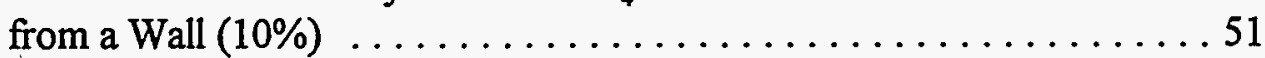

Figure 4.4.1-1. Single Fully Reflected 5.00" $\mathrm{D}$ x 24" Tall Cylinder of $\mathrm{UO}_{2} \mathrm{~F}_{2} \ldots \ldots \ldots 54$

Figure 4.4.1-2. Single 5.00" $\mathrm{D} \times 24$ " Tall Cylinder of $\mathrm{UO}_{2} \mathrm{~F}_{2}$ in a Concrete Corner ...... 55

Figure 4.4.1-3. Two 5.00" ID x 24" Tall Cylinders of $\mathrm{UO}_{2} \mathrm{~F}_{2}$ Next to a Wall ......... 56

Figure 4.4.1-4. Two 5.00" ID x 24" Tall Cylinders of $\mathrm{UO}_{2} \mathrm{~F}_{2}$ One Foot from a Wall ...... 57 


\section{LIST OF FIGURES (continued)}

Figure 4.4.1-5. Single Fully Reflected 5.00" $\mathrm{DD}$ x 24" Tall Cylinder of $\mathrm{UF}_{4}$ and Oil . ....58 Figure 4.4.1-6. Single 5.00" $\mathrm{ID} \times 24^{\prime \prime}$ Tall Cylinder of $\mathrm{UF}_{4}$ and Oil in a Concrete Corner . . 59 Figure 4.4.1-7. Two 5.00" $\mathrm{D}$ x 24" Tall Cylinders of $\mathrm{UF}_{4}$ and Oil Next to a Wall ......6 60 Figure 4.4.1-8. Two 5.00" $\mathrm{D}$ x 24" Tall Cylinders of $\mathrm{UF}_{4}$ and Oil One Foot from a Wall . . 61 Figure 4.4.1-9. An Array of 5.00" $\mathrm{ID} \mathrm{x} 24^{\prime \prime}$ Tall Cylinders of $\mathrm{UO}_{2} \mathrm{~F}_{2} \ldots \ldots \ldots \ldots \ldots 6$ Figure 4.4.1-10. An Array of 5.00" $\mathrm{ID} \times 24$ " Tall Cylinders of $\mathrm{UF}_{4}+$ Oil . . . . . . . . 63 Figure 4.4.1-11. The Effects of Interstitial Water Volume Fraction on $\mathrm{k}_{\mathrm{eff}} 5.00^{\prime \prime} \mathrm{UO}_{2} \mathrm{~F}_{2} \ldots .64$ Figure 4.4.1-11a. The Effects of Interstitial Water Volume Fraction on $\mathrm{k}_{\text {eff }} 5.00^{\prime \prime} \mathrm{UO}_{2} \mathrm{~F}_{2} \ldots \ldots 65$ Figure 4.4.1-12. An Array of 5.00" $\mathrm{D} \mathrm{x} 24$ " Tall Cylinders of $\mathrm{UO}_{2} \mathrm{~F}_{2} \ldots \ldots \ldots \ldots 66$ Figure 4.4.2-1. Single Fully Reflected 5.00" ID x 24" Tall Cylinder of $\mathrm{UO}_{2} \mathrm{~F}_{2}(10 \%) \ldots \ldots 68$ Figure 4.4.2-2. Single 5.00" $\mathrm{ID} \times 24$ " Tall Cylinder of $\mathrm{UO}_{2} \mathrm{~F}_{2}$ in a Concrete Corner (10\%) . 69

Figure 4.4.2-3. Two 5.00" ID x 24" Tall Cylinders of $\mathrm{UO}_{2} \mathrm{~F}_{2}$ Next to a Wall $(10 \%) \ldots . .70$ Figure 4.4.2-4. Figure 4.4.2-5. Figure 4.4.2-6.

Figure 4.4.2-7. Figure 4.4.2-8.

Figure 4.4.3-1. Figure 4.5.1-1. Figure 4.5.1-2. Figure 4.5.1-3. Figure 4.5.1-4. Figure 4.5.1-5. Figure 4.5.1-6. Figure 4.5.1-7. Figure 4.5.1-8. Figure 4.5.1-9. Figure 4.5.2-1. Figure 4.5.2-2. Figure 4.5.2-3. Figure 4.5.2-4. Figure 4.5.2-5. Figure 4.5.2-6.

Figure 4.5.2-7. Figure 4.5.2-8. Single Fully Reflected 5.00" ID $\times 24^{\prime \prime}$ Tall Cylinder of

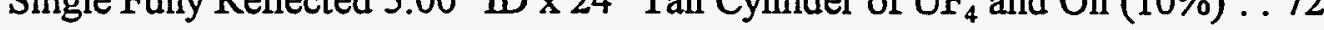

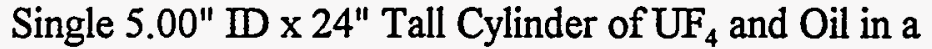
Concrete Corner $(10 \%) \ldots \ldots \ldots \ldots \ldots \ldots \ldots \ldots \ldots \ldots \ldots \ldots$ Two 5.00" ID x 24" Tall Cylinders of $\mathrm{UF}_{4}$ and Oil Next to a Wall (10\%) . 74 "Two 5.00" ID x 24" Tall Cylinders of $\mathrm{UF}_{4}$ and Oil One Foot

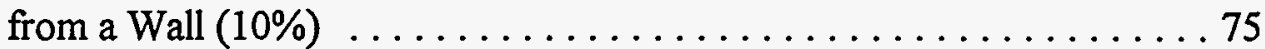
Single 5.00" ID x 24" Tall Cylinder of $\mathrm{UF}_{4}$ and Oil ............ 77 Single Fully Reflected 5.25" ID x 24" Tall Cylinder of $\mathrm{UO}_{2} \mathrm{~F}_{2} \ldots \ldots \ldots .79$ Single 5.25" ID x 24" Tall Cylinder of $\mathrm{UO}_{2} \mathrm{~F}_{2}$ in a Concrete Corner . . . . . 80 Two 5.25" ID x 24" Tall Cylinders of $\mathrm{UO}_{2} \mathrm{~F}_{2}$ Next to a Wall ........ 81 Two 5.25" ID x 24" Tall Cylinders of $\mathrm{UO}_{2} \mathrm{~F}_{2}$ One Foot from a Wall .... 82 Single Fully Reflected 5.25" I x 24" Tall Cylinder of $\mathrm{UF}_{4}$ and Oil ..... 83 Single 5.25" $\mathrm{DD} \times 24^{\prime \prime}$ Tall Cylinder of $\mathrm{UF}_{4}$ and Oil in a Concrete Corner . . 84 Two 5.25" ID x 24" Tall Cylinders of $\mathrm{UF}_{4}$ and Oil Next to a Wall ..... 85 Two 5.25" ID x 24" Tall Cylinders of $\mathrm{UF}_{4}$ and Oil One Foot from a Wall . . 86 Single 5.25" $\mathrm{ID} \times 24$ " Tall Cylinder of $\mathrm{UF}_{4}$ and Oil ............ 87 Single Fully Reflected 5.25" ID x 24" Tall Cylinder of $\mathrm{UO}_{2} \mathrm{~F}_{2}(10 \%) \ldots .89$ Single 5.25" ID x 24" Tall Cylinder of $\mathrm{UO}_{2} \mathrm{~F}_{2}$ in a Concrete Corner (10\%) . 90 Two 5.25" ID x 24" Tall Cylinders of $\mathrm{UO}_{2} \mathrm{~F}_{2}$ Next to a Wall (10\%) .... 91 Two 5.25" ID x 24" Tall Cylinders of $\mathrm{UO}_{2} \mathrm{~F}_{2}$ One Foot from a Wall (10\%) . 92 Single Fully Reflected 5.25" ID x 24" Tall Cylinder of $\mathrm{UF}_{4}$ and Oil (10\%) . 93 Single 5.25" ID x 24" Tall Cylinder of $\mathrm{UF}_{4}$ and Oil in a Concrete Corner $(10 \%) \ldots \ldots \ldots \ldots \ldots \ldots \ldots \ldots . \ldots \ldots$ Two 5.25" ID x 24" Tall Cylinders of $\mathrm{UF}_{4}$ and Oil Next to a Wall (10\%) . . 95 Two 5.25" ID x 24" Tall Cylinders of $\mathrm{UF}_{4}$ and Oil One Foot from a Wall $(10 \%) \ldots \ldots \ldots \ldots \ldots \ldots \ldots \ldots . . . \ldots 6$ 


\section{LIST OF FIGURES (continued)}

Figure 4.6-1. Single Fully Reflected 5.25" $\mathrm{ID} \mathrm{x} \mathrm{15"} \mathrm{Tall} \mathrm{Cylinder} \mathrm{of} \mathrm{UO}_{2} \mathrm{~F}_{2} \ldots \ldots \ldots 98$

Figure 4.6-2. Single Fully Reflected 5.25" $\mathrm{ID} \mathrm{x} \mathrm{15"} \mathrm{Tall} \mathrm{Cylinder} \mathrm{of} \mathrm{UF}_{4}$ and Oil . . . . . . 99

Figure 4.6-3. Two 5.25" ID x 15" Tall Cylinders of $\mathrm{UO}_{2} \mathrm{~F}_{2}$ One Foot from a Wall ... 100

Figure 4.6-4. Single 5.25" ID x 15" Tall Cylinder of $\mathrm{UF}_{4}$ and Oil ............. 101

Figure 4.6-5.

Figure 4.6-6.

Figure 4.6-7. Single Fully Reflected 5.25" ID x 15" Tall Cylinder of $\mathrm{U}_{3} \mathrm{O}_{8} \ldots \ldots \ldots 102$

Figure 4.6-8. Single 5.25" ID x 15" Tall Cylinder of $\mathrm{U}_{3} \mathrm{O}_{8}$ in a Concrete Corner . . . . . . 103

Figure 4.6-9.

Figure 4.6-10. Two 5.25" ID x 15" Tall Cylinders of $\mathrm{U}_{3} \mathrm{O}_{8}$ Next to a Wall .......... 104 Two 5.25" ID x 15" Tall Cylinders of $\mathrm{U}_{3} \mathrm{O}_{8}$ One Foot from a Wall ..... 105 Figure 4.6-10a. Figure 4.6-11.

Figure 4.7-1.

Figure 4.7-2. An Array of 5.25" I x 15" Tall Cylinders of $\mathrm{U}_{3} \mathrm{O}_{8} \ldots \ldots \ldots \ldots \ldots 106$ The Effects of Interstitial Water Volume Fraction on $\mathrm{k}_{\text {eff }} 5.25^{\prime \prime} \mathrm{U}_{3} \mathrm{O}_{8} \ldots \ldots 107$

Figure 4.7-3. The Effects of Interstitial Water Volume Fraction on $\mathrm{k}_{\mathrm{eff}} 5.25^{\prime \prime} \mathrm{U}_{3} \mathrm{O}_{8} \ldots 108$ An Array of 5.25" ID x 15" Tall Cylinders of $\mathrm{U}_{3} \mathrm{O}_{8}$. . . . . . . . . . . 109 Single Fully Reflected 5.00" ID x 9" Poly. Container of $\mathrm{U}_{3} \mathrm{O}_{8} \ldots \ldots \ldots 111$

Figure 4.7-4. Single 5.00" ID x 9" Tall Cylinder of $\mathrm{U}_{3} \mathrm{O}_{8}$ in a Concrete Corner ...... 112 Two 5.00" $\mathrm{ID} \times 9^{\prime \prime}$ Tall Cylinders of $\mathrm{U}_{3} \mathrm{O}_{8}$ Next to a Wall ..........113

Figure 4.7-5. Two 5.00" ID x 9" Tall Cylinders of $\mathrm{U}_{3} \mathrm{O}_{8}$ One Foot from a Wall ..... 114

Figure 4.8-1. Single Fully Reflected $5.00^{\prime \prime} \mathrm{ID} \times 24$ " Tall Cylinder of $\mathrm{U}_{3} \mathrm{O}_{8} \ldots \ldots \ldots 115$

Figure 4.8-2. - An Array of 4.32" ID x 50" Tall Cylinders of $\mathrm{UF}_{4}+\mathrm{Oil}(10 \%) \ldots \ldots \ldots 118$

Figure 4.8-3. An Array of 5.25" ID x 24" Tall Cylinders of $\mathrm{UF}_{4}+\mathrm{Oil}(10 \%) \ldots \ldots \ldots 119$

Figure 4.8-4.

Figure 4.8-5. An Array of 5.00" ID x 24" Tall Cylinders of $\mathrm{UF}_{4}+\mathrm{Oil}(10 \%) \ldots \ldots \ldots 120$ Two 5.25" ID x 24" Tall Cylinders of $\mathrm{UF}_{4}+$ Oil Side by Side Fully Reflected (10\%)

Figure 4.8-6. An Array of 5.00" ID x 24" Cylinders of $\mathrm{UF}_{4}+$ Oil 1' Spacing . . . . . . . 122 An Array of 5.25" ID x 24" Tall Cylinder of $\mathrm{UF}_{4}$ and Oil Adjacent to Concrete Walls ............................ 123 


\section{LIST OF TABLES}

Table 1.0-1. Small-Diameter Uranium-Bearing Containers . . . . . . . . . . . . . 2

Table 3.2-1. Number Densities (atoms/barn-cm) for $\mathrm{UF}_{4}+$ Oil for $100 \%$ Enriched Uranium ............................. 16

Table 3.2-2. Number Densities (atoms/barn-cm) for $\mathrm{UF}_{4}+$ Oil for $10 \%$ Enriched Uranium ............................ 17

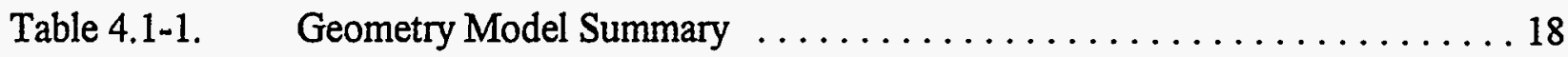

Table 4.1.2-0. Container Thickness Result Summary ................... 20

Table 4.1.4-1. GP Container Model Assumptions Summary Table ............ 25

Table 4.3.1-0. Summary of 4.32-inch-Diameter Container Results for 100\% Enrichment . . 26

Table 4.3.2-0. Summary of 4.32-inch Container Results for $10 \%$ Enrichment . . . . . . . 43

Table 4.4.1-0. Summary of 5.0-inch References for $100 \%$ Enrichment . . . . . . . . . . 53

Table 4.4.2-0. Summary of 5.0-inch Bottle References for $10 \%$ Enrichment . . . . . . . 67

Table 4.5.1-0. Summary of References for F-Can Results for $100 \%$ Enrichment . . . . . 78

Table 4.5.2-0. Summary of References for F-Can Results for $10 \%$ Enrichment $\ldots \ldots \ldots 88$

Table 4.6-0. Summary of References for $Z$-Can Results $\ldots \ldots \ldots \ldots \ldots \ldots$

Table 4.7-0. Summary of References for CO-4 Polybottles . . . . . . . . . . . . . . 110

Table 4.9-1. Summary of References for $100 \%$ Enriched Material . . . . . . . . . . . . 124 
POEF-SH-30

\subsection{INTRODUCTION}

This report documents calculations performed to establish a technical basis for the nuclear criticality safety of favorable geometry containers, sometimes referred to as 5-inch containers, in use at the Portsmouth Gaseous Diffusion Plant. A list of containers currently used in the plant is shown in Table 1.0-1. These containers are currently used throughout the plant with no mass limits. The use of containers with geometries or material types other than those addressed in this evaluation must be bounded by this analysis or have an additional analysis performed. The following five basic container geometries were modeled and bound all container geometries in Table 1.0-1:

1. 4.32-inch-diameter by 50 -inch-high polyethylene bottle

2. 5.0-inch-diameter by 24-inch-high polyethylene bottle

3. 5.25-inch-diameter by 24 -inch-high steel can ("F-can")

4. 5.25-inch-diameter by 15 -inch-high steel can ("Z-can")

5. $\quad 5.0$-inch-diameter by 9 -inch-high polybottle ("CO-4")

Each container type is evaluated using five basic reflection and interaction models that include single containers and multiple containers in normal and in credible abnormal conditions. The uranium materials evaluated are $\mathrm{UO}_{2} \mathrm{~F}_{2}+\mathrm{H}_{2} \mathrm{O}$ and $\mathrm{UF}_{4}+$ oil materials at $100 \%$ and $10 \%$ enrichments and $\mathrm{U}_{3} \mathrm{O}_{8}$ and $\mathrm{H}_{2} \mathrm{O}$ at $100 \%$ enrichment. The design basis safe criticality limit for the Portsmouth facility is $k_{\text {eff }}+2 \sigma<0.95$. The KENO study results may be used as the basis for evaluating general use of these containers in the plant. 
POEF-SH-30

Table 1.0-1. Small-Diameter Uranium-Bearing Containers

\begin{tabular}{|c|c|c|c|c|}
\hline Container Type & References & $\begin{array}{c}\text { Inner Diameter } \\
\text { (inches) }\end{array}$ & $\begin{array}{l}\text { Height } \\
\text { (inches) }\end{array}$ & $\begin{array}{l}\text { Wall Thickmess } \\
\text { (inches) }\end{array}$ \\
\hline "744G" Polyethylene Bottle & (DWG. DX-761-2340-M, Rev.0) & $\begin{array}{c}4.25 \\
(+/-0.07-\mathrm{in})\end{array}$ & 50.373 & $\begin{array}{c}0.125 \\
(+.07,-.000)\end{array}$ \\
\hline "24-in Polybottle" Polyethylerie Bottle & (DWG. BX-761-M373 Rev 0) & 5.0 & 24.0 & Min. 0.1875 \\
\hline $\begin{array}{l}\text { "OG Container" Polyethylene } \\
\text { Container (Conceptual) }\end{array}$ & Not Available & 4.25 & 24.0 & Not Available \\
\hline $\begin{array}{c}\text { "CO-4 Polybottles" Polyethylene } \\
\text { Bottle (DWG Not Available) }\end{array}$ & Not Available & 5.0 & 9.0 & Not Available \\
\hline "F-Can" Tin Plated Steel Container & (DWG. DX-761-M-981) & 5.25 & 24.0 & Min. 0.015 \\
\hline "Z-Can" Tin Plated Steel Container & (DWG. DX-761-2331-M) & 5.25 & 15.0 & Min. 0.015 \\
\hline
\end{tabular}


POEF-SH-30

\subsection{ANALYSIS ASSUMPTIONS}

\subsection{Models}

All calculations were performed on the Battelle Process Safety and Risk Management IBM RS/6000 workstation implementation of SCALE-4.2. The validation performed on this software/hardware combination and its area of applicability are discussed in Validation of the CSAS25 Calculation Sequence in Scale-4.2 and the 27 Energy Group ENDF/B-IV Cross Sections on the Battelle Process Safety and Risk Management IBM RS/6000 Workstation (Ref. 6) and Conditional Validation of CSAS25 on Battelle Process Safety and Risk Management RS/6000 (Ref. 7). Configuration control of this software/hardware combination is documented in Configuration Control Plan for Process Safety and Risk Management Nuclear Safety Software \& Hardware Systems (IBM/RS6000) (Ref. 8). The SCALE system of computer codes is a large collection of modularized computer codes useful for performing a variety of nuclear safety calculations, including criticality calculations. The results presented in the following sections were all obtained using the CSAS25 calculational sequence. The CSAS25 modules consist of BONAMI, NITAWL, and KENO Va. The BONAMI and NITAWL adjust the cross-sections data for the specific problem at hand (e.g., performance of resonance self-shielding corrections). The 27 energy group cross-sections available in SCALE were used for all calculations. The critical experiment that validates the code for $\mathrm{UF}_{4}$ +oil is CAS14 (TM-238) (Ref. 6).

Validation Argument: The validaton report (Ref . 7) states that for the vast majority of fissile systems found at Portsmouth Gaseous Diffusion Plant, it is appropriate to use the CSAS25 sequence of SCALE-4.2 to analyze them. The different types of low-enriched systems for which critical experiments were preformed include uranium metal at enrichments of $3.85 \%$ and $4.89 \%$, moderated and reflected by $\mathrm{H}_{2} \mathrm{O} ; \mathrm{U}_{3} \mathrm{O}_{8}$ sterotex blocks at $4.89 \%$, both reflected and 
unreflected by $\mathrm{H}_{2} \mathrm{O} ; \mathrm{UO}_{2} \mathrm{~F}_{2}$ solution at $4.89 \%$ enrichment, and $\mathrm{U}^{235}$ densities ranging from approximately 22.1 to $42.5 \mathrm{gU}^{235} / 1$; and $\mathrm{UF}_{4}$ (paraffin) systems at enrichments of $1.4 \%$ to $3 \%, \mathrm{H} / \mathrm{X}$ ratios from 133 to 971 , reflected and unreflected, to simulate $\mathrm{UF}_{6}$. The highly enriched systems include $\mathrm{UO}_{2} \mathrm{~F}_{2}$ solutions at approximately $93 \%$, with solutions at enrichments of $93.2 \%$ with uranium densities ranging from 55 to $364 \mathrm{gU} / 1$, reflected and unreflected; and uranium metal systems between $93 \%$ and $97 \%$, enrichment unreflected. The validation covers all the materials analyzed in this report.

The enrichment of $\mathrm{UF}_{4}+$ oil analyzed in this report is $100 \%$ while the validation of a similar material $\mathrm{UF}_{4}$ (paraffin) was performed at a maximum of $3 \%$ (Ref. 6.0).

Highly enriched uranium metal, and $\mathrm{UO}_{2} \mathrm{~F}_{2}$ systems have been validated; therefore, the confidence of the highly enriched $\mathrm{UF}_{4}+$ oil cases remains within the $\mathrm{k}_{\text {eff }}+2 \sigma<0.95$ calculational acceptance criteria.

Each of the geometry options used to model the systems analyzed in this report has been validiated in Reference 7 .

\subsubsection{Description of Standard Geometry Models}

Each of the containers discussed in this document is analyzed in accordance with Standards for Analysis of Small Containers at the Portsmouth Plant (Ref. 9), which defines the standard reflection models to be used when evaluating smallcontainer nuclear criticality safety. Each of the geometry models from this reference is described in detail in the following sections and is assigned an acronym for identification purposes. The Appendix contains sample input files. 


\subsubsection{Standard Room (SR)}

This model applies to all cases with the exception of the full water reflection case, which does not require the addition of any external reflector. The basic conditions include

Floor: 24-inch-thick regular concrete floor

Ceiling: $\quad$ 12-inch-thick regular concrete ceiling (10 feet high)

Walls: 12-inch-thick concrete walls

When any of the above described surfaces is in contact with the container being analyzed, the concrete surface is extended away from the container at least a distance equal to the height of the fuel/container from that surface.

\subsubsection{Single Container Standard Reflection Models}

Full Reflection (SFR). Each of the containers is analyzed as a single container under conditions of full water reflection. The container is modeled as sitting on a standard room floor with 12 inches of close-fitting, full-density water on all other surfaces (around container and top).

In a Concrete Corner (SCC). A single container is placed in a concrete corner in contact with two walls and the floor. The model includes contact by the person handling the container. The person is modeled as $\sim 100 \mathrm{~kg}$ of water that is L-shaped around the container as shown in Figure 2.1.3-1. The height of the water reflector is 50 inches for the 4.32 -inch containers and 48 inches for 5.0-inch and 5.25-inch containers. 

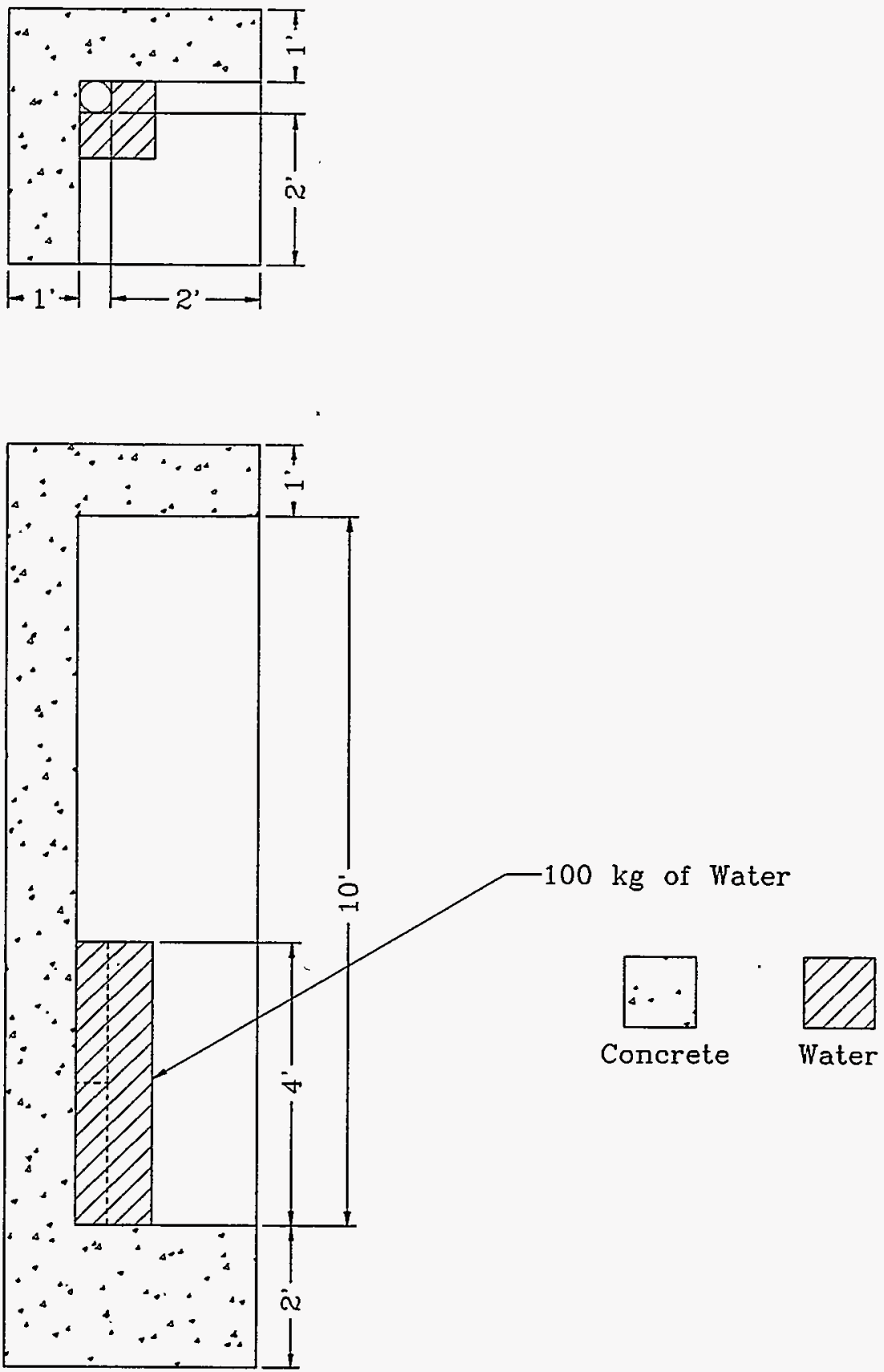

Figure 2.1.3-1. Single Container in a Corner with Water Reflector Simulating a Person 
More Reactive Contents (SMRC). The standard material is replaced with a more reactive material present in the facility. The container is modeled assuming 24 inches of concrete on the bottom and 1 inch of close-fitting water reflection on the other surfaces.

\subsubsection{Two Containers Touching Reflection Models}

Adjacent to a Concrete Wall (TACW). The model simulates two containers touching in a position adjacent to a concrete wall and reflected by a person. The person is modeled as a $\sim 100 \mathrm{~kg}, 18$-inch-wide by 7-inch-deep by 4 -foot-high block with hands modeled as 1-inch-thick slabs against the outside of the containers as shown in Figure 2.1.4-1. This analysis assumes no storage locations close to the containers. The model also incorporates the standard room reflection conditions.

One Foot From a Concrete Wall (TSCW). This model is the same as the previous model with the exception that the two containers are moved a distance of one foot from the concrete wall. This configuration is represented in

Figure 2.1.4-2.

\subsubsection{Array Models}

Arrays in a Concrete Enclosure (ACE). This standard configuration models four double rows of containers located in a concrete enclosure (the concrete floor, ceiling, and wall thickness are described in Section 2.1.2). A double row consists of 2 rows of containers separated by 23 inches center-to-center. The model includes the maximum polyethylene bottle thickness of 0.195 inches for the bottles but does not include the steel container thickness for the 5.25-inch diameter $\mathrm{F}$ and $Z$ Cans. The rows are 40 units long. Each double row is separated by 6 -foot aisles, and the array is a minimum of 2 feet from the concrete walls. A sketch of the standard array model is shown in Figure 2.1.5-1. 

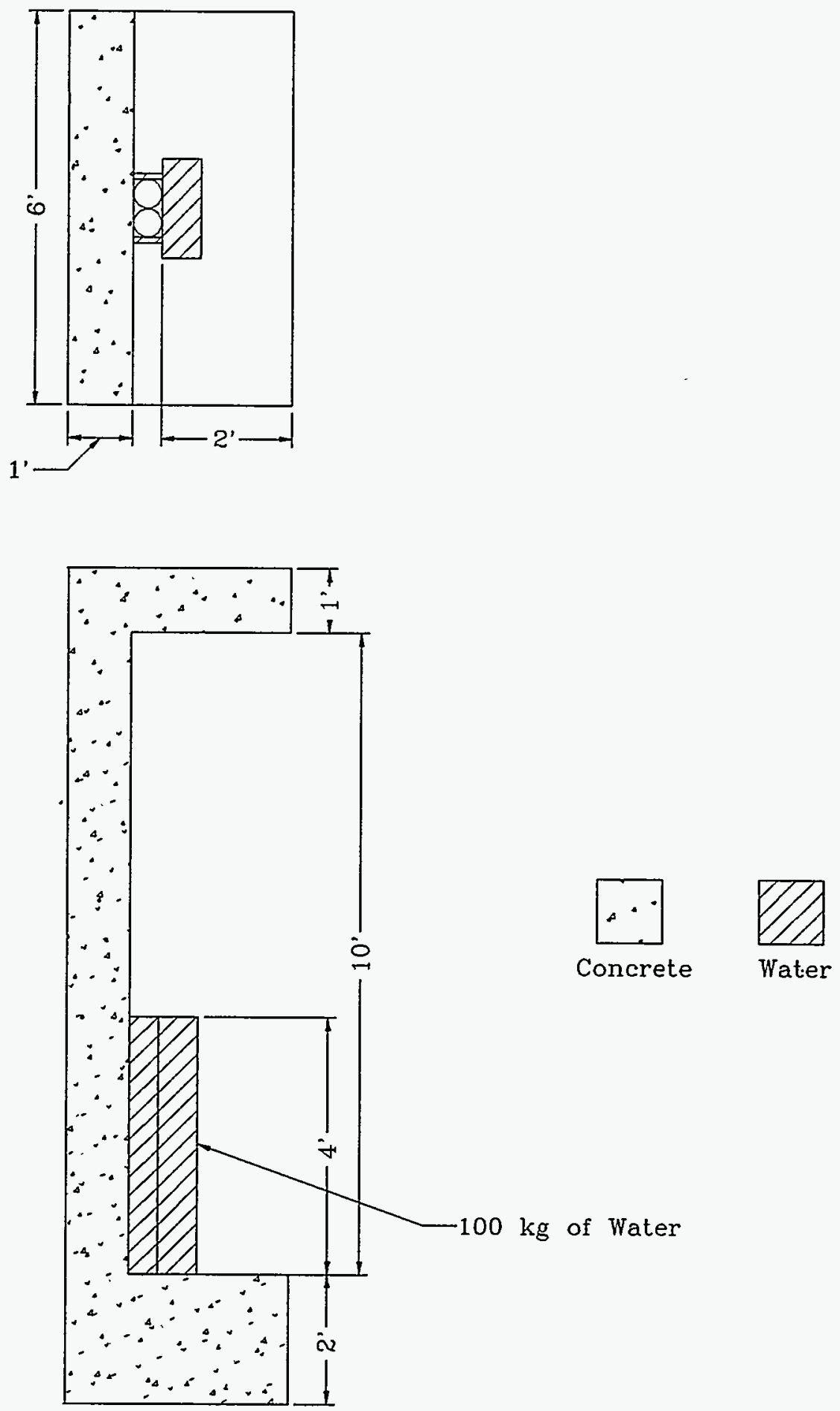

Figure 2.1.4-1 Two Containers Next to a Wall with Water Reflector Simulating a Person 


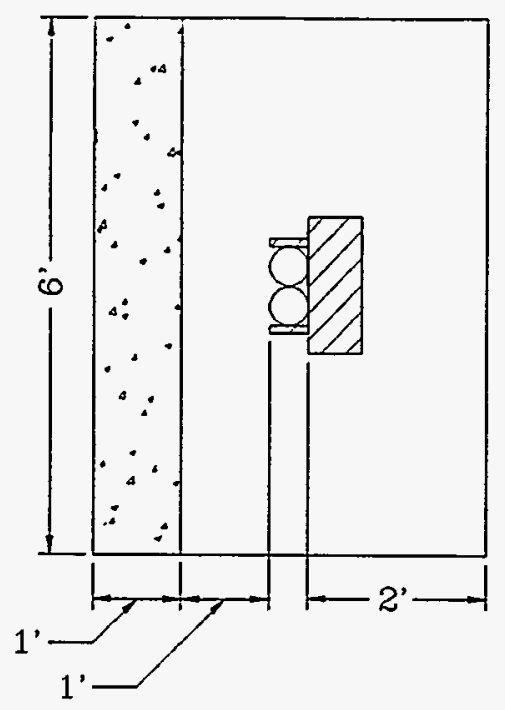

POEF-SH-30

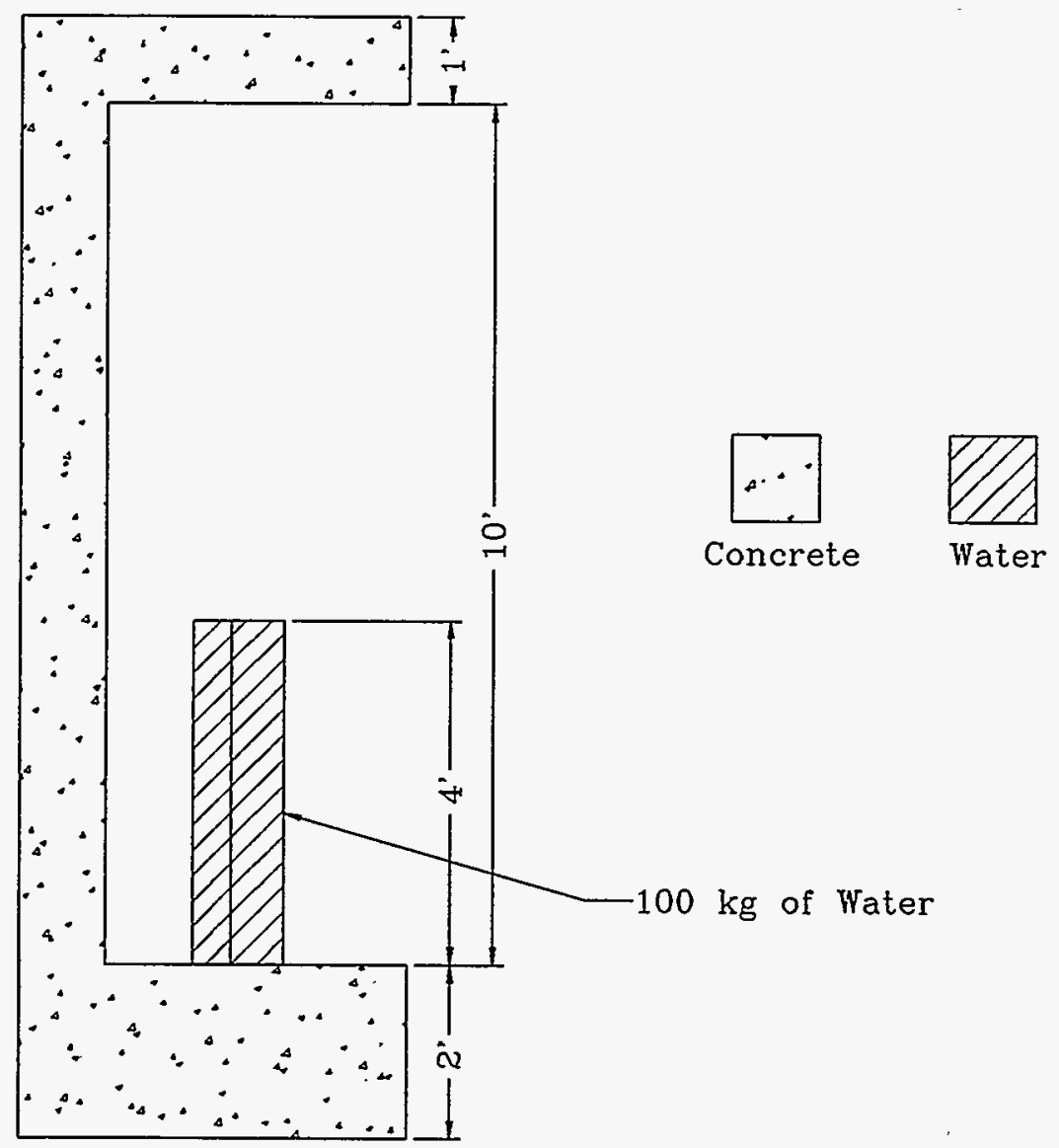

Figure 2.1.4-2. Two Containers 1-Foot from a Wall with Water Reflector Simulating a Person 


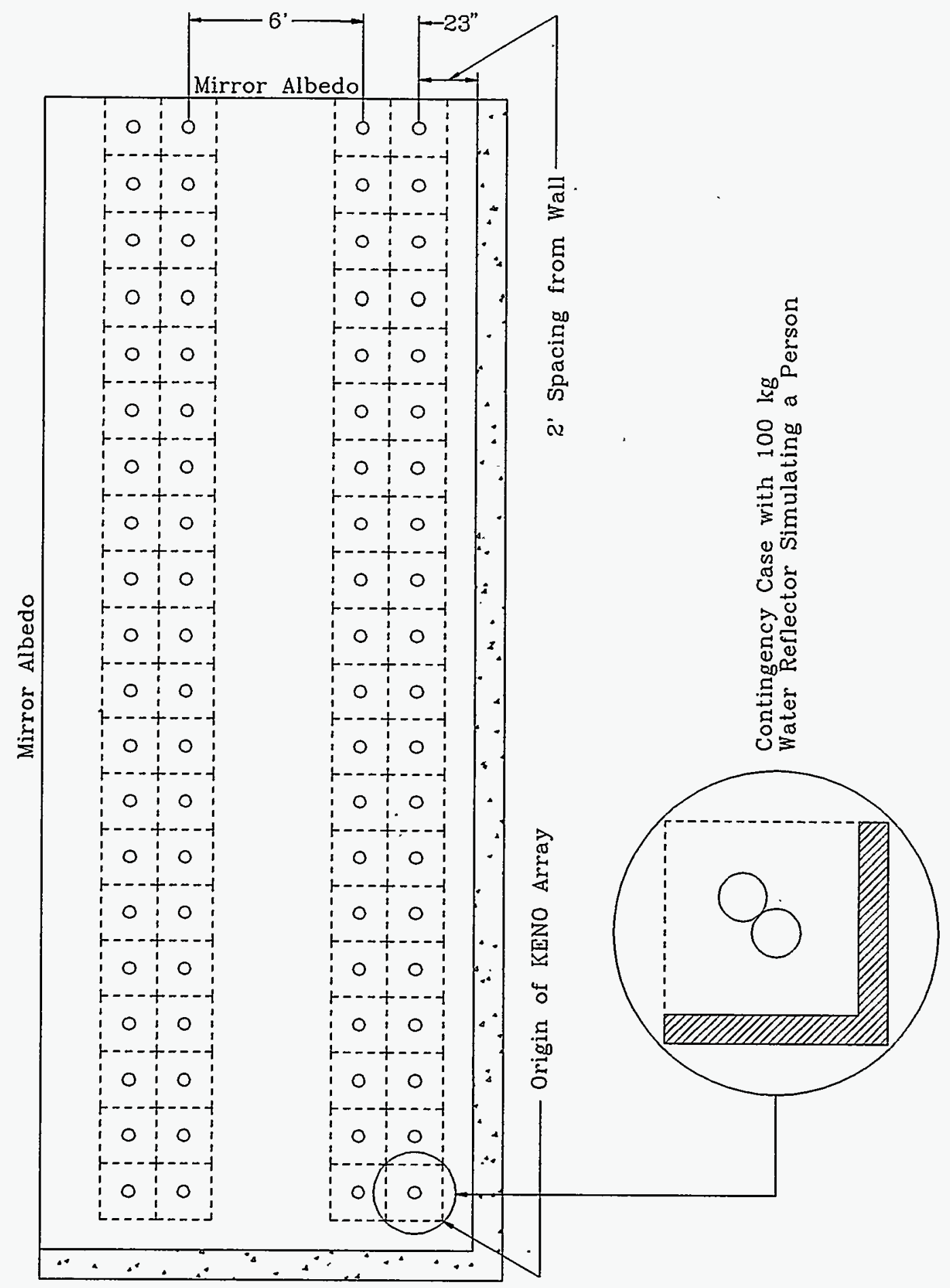

Figure 2.1.5-1. Standard Array Configuration with Concrete Reflector 
POEF-SH-30

Interstitial Moderation (AD). This model analyzes the effect of adding interstitial moderation to the spaces separating the units in the standard array model (ACE). The optimum $\mathrm{H} / \mathrm{U}$ ratio from the standard model and $\mathrm{H} / \mathrm{U}$ values above and below this peak are evaluated for a range of water densities. The water density that produces the peak $\mathrm{k}_{\text {eff }}$ is compared with the physical limits of the sprinkler system or other sources of moderation. Each of the container types is evaluated using this model.

\subsection{Uranium Compounds Analyzed}

Uranium is present in many chemical forms at the Portsmouth Plant. The scope of this analysis is limited to the following three material types: $\mathrm{UO}_{2} \mathrm{~F}_{2} /$ water, $\mathrm{UF}_{4} /$ oil, and $\mathrm{U}_{3} \mathrm{O}_{8}$.

\subsection{1 $\mathrm{UO}_{2} \mathrm{~F}_{2}+\mathrm{H}_{2} \mathrm{O}$}

Of the chemical forms present, $\mathrm{UO}_{2} \mathrm{~F}_{2}+\mathrm{H}_{2} \mathrm{O}$ is of primary concern because of its high uranium solubility and it is the most common material handled.

\subsection{2 $\mathrm{UF}_{4}+\mathrm{Oil}$}

The $\mathrm{UF}_{4}$ +oil primarily is present in vacuum pump lubricating oil that has been in contact with $\mathrm{UF}_{6}$ in the gaseous diffusion process and is analyzed because the reactivity is potentially higher than $\mathrm{UO}_{2} \mathrm{~F}_{2}+\mathrm{H}_{2} \mathrm{O}$. The composition of oil used in the analysis was assumed to be $\mathrm{CH}_{2}$ with an assumed density of $0.87 \mathrm{~g} / \mathrm{cc}$ (Ref. 10). The $\mathrm{H} / \mathrm{C}$ ratio varies with oil type with typical oil having an $\mathrm{H} / \mathrm{C}$ ratio of approximately 2.0. The reaction is discussed in Reference 10 .

\subsection{3 $\quad \mathrm{U}_{3} \mathrm{O}_{8}$}

The $\mathrm{U}_{3} \mathrm{O}_{8}$ is produced in the calciner by exposing uranyl nitrate solutions to high-temperature air, which results in the removal of water and the subsequent conversion to $\mathrm{U}_{3} \mathrm{O}_{8}$. Calciner operations are located in Building $\mathrm{X}-705$. The theoretical density of $\mathrm{U}_{3} \mathrm{O}_{8}$ is $8.21 \mathrm{gU} / \mathrm{cc}$ (Ref. 16). The $\mathrm{UO}_{2}$ is not considered a product from the calciners, because the physical conditions required to convert 
uranyl nitrate to $\mathrm{UO}_{2}$ are not achieved under normal operating conditions.

Conversion to $\mathrm{UO}_{2}$ requires a reducing agent such as $\mathrm{H}_{2}$, which is not present in the calciner design (Ref. 18). Therefore, producing $\mathrm{UO}_{2}$ and storing it in a Z-Can or CO-4 polybottle is considered a credible contingency. 


\subsection{DETERMINATION OF NUMBER DENSITIES}

\section{$3.1 \quad \mathrm{UO}_{2} \mathrm{~F}_{2}$}

\subsubsection{Determination of Number Density}

Uranyl fluoride $\left(\mathrm{UO}_{2} \mathrm{~F}_{2}\right)$ is a hygroscopic material. In high-moisture environments and at moderate temperatures, $\mathrm{UO}_{2} \mathrm{~F}_{2}$ can absorb moisture and form an aqueous solution. The $\mathrm{H} / \mathrm{U}$ ratios of over 30 can be reached when initially dry material is exposed to humid air (Ref. 11). For this reason, all $\mathrm{UO}_{2} \mathrm{~F}_{2}$ cases assume the presence of $\mathrm{H}_{2} \mathrm{O}$. The number densities determined in this analysis of the various $\mathrm{UO}_{2} \mathrm{~F}_{2}$ and water mixtures were calculated through the utilization of the existing program NUMDEN (Ref. 12). NUMDEN is a FORTRAN code capable of both computing densities for a host of mixtures of uranium and moderating materials as well as atomic number density data. This information then can be used as input parameters in the KENO Va code for the determination of $k_{\text {eff }}$ NUMDEN can perform number density calculations using a variety of correlations. This analysis uses the Barber method to calculate the uranium densities for the $\mathrm{UO}_{2} \mathrm{~F}_{2}$ solutions investigated in this report. The Barber method is composed of the following two equations:

$\rho_{u}=4.96-0.32(H / U)$

For $\mathrm{H} / \mathrm{U}<4$

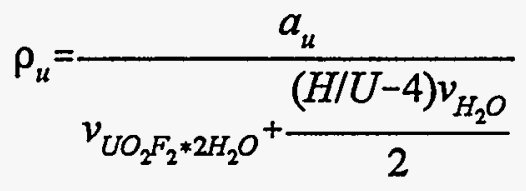

For $\mathrm{H} / \mathrm{U} \geq 4$ 


\subsubsection{Justification for Using a Uranium Density of $3.2 \mathrm{~g} / \mathrm{cc}$ for $\mathrm{H} / \mathrm{U}$ Less Than 4.0} The application of the Barber relationship (Ref. 12) for $\mathrm{H} / \mathrm{U}$ values less than 4 produces a highly conservative uranium density. The high uranium density at an $\mathrm{H} / \mathrm{U}$ of 4 results in a discontinuous maximum peak in $\mathrm{k}_{\text {eff }}$ for several of the cases tested. The peak is the only $\mathrm{H} / \mathrm{U}$ point that exceeds the limiting condition in several of the contingencies tested.

The theoretical density of $\mathrm{UO}_{2} \mathrm{~F}_{2}$ is $6.37 \mathrm{~g} / \mathrm{cc}(\operatorname{Ref} 13)$, which produces a uranium density of $4.97 \mathrm{~g} / \mathrm{cc}$. In an unsaturated atmosphere and at moderate temperatures, . uranyl fluoride $\left(\mathrm{UO}_{2} \mathrm{~F}_{2}\right)$ will absorb moisture and form hydrates. The stable hydrate $\mathrm{UO}_{2} \mathrm{~F}_{2}{ }^{*} 2 \mathrm{H}_{2} \mathrm{O}$ is the most likely to be formed. Historically, the gaseous diffusion plants have used a maximum uranium density of $3.2 \mathrm{gU} / \mathrm{cc}$ (Ref. 17). Undisturbed cascade deposits may approach theoretical density. However, once disturbed by mechanical recovery or exposure to ambient air, $\mathrm{UO}_{2} \mathrm{~F}_{2}$ behaves as a bulk powder even if dried back to the anhydrous state. The use of $3.2 \mathrm{gU} / \mathrm{cc}$ as a maximum uranium density for deposits removed from cascade equipment is considered to conservatively bound the actual bulk density of $\mathrm{UO}_{2} \mathrm{~F}_{2}$.

\section{2 $\quad \mathrm{UF}_{4}+$ Oil Number Density Determination}

The $\mathrm{UF}_{4}+$ oil is present at the Portsmouth Plant in contaminated equipment. The volume-additive method was used to calculate number density (Ref. 10). The following information is required to perform this calculation:

$$
\begin{aligned}
& \left.\mathrm{UF}_{4} \text { Density }=6.70 \mathrm{~g} / \mathrm{cm}^{3} \text { (Ref. } 15\right) \\
& \text { Oil Density }=0.87 \mathrm{~g} / \mathrm{cm}^{3}(\text { Ref. } 10) \\
& \text { MW Fluorine }=18.99 \mathrm{~g} / \text { mole (Ref. } 14) \\
& \text { MW Uranium }=235 \mathrm{~g} / \text { mole (Ref. } 14, \text { assumed to be } 100 \% \text { enriched) } \\
& \text { MW Carbon }=12.011 \mathrm{~g} / \text { mole (Ref. } 14) \\
& \text { MW Hydrogen }=1.00 \text { (Ref. } 14)
\end{aligned}
$$


Avogadro's Number $=0.602214 \mathrm{E}+24$ atom $/$ mole (Ref. 14) $\mathrm{cm}^{2}$ to barns conversion multiply by $1 \mathrm{E}-24$

The equation for calculating number density is

$$
\mathrm{ND}=[(\mathrm{MD})(\mathrm{AV})] / \mathrm{MW}
$$

where

$\mathrm{ND}=\quad$ Number Density (atoms/barn-cm)

$\mathrm{MD}=\quad$ Element Density in the mixture $\left(\mathrm{g} / \mathrm{cm}^{3}\right)$

$\mathrm{AV}=\quad$ Avogadro's Number multiplied by the conversion factor (atoms$\mathrm{cm}^{2} /$ barn-mole)

$M W=\quad$ Molecular Weight of the Element $(\mathrm{g} / \mathrm{mole})$

To calculate the density of each element in the compound, the ratio of the molecular weight of the element and the molecular weight of the compound is multiplied by the product of the compound density and the volume fraction. Once the number density for each element is determined, the $\mathrm{H} / \mathrm{U}$ ratio can be determined. The $\mathrm{H} / \mathrm{U}$ ratio is simply the ratio of the hydrogen number density to the uranium number density. Table 3.2-1 shows the computed values for the number densities of the $\mathrm{UF}_{4}+$ oil case for $100 \%$ uranium enrichment, and Table 3.2-2 lists the number densities for the $10 \%$ uranium enrichment case. 
POEF-SH-30

Table 3.2-1. Number Densities (atoms/barn-cm) for $\mathrm{UF}_{4}+$ Oil for $100 \%$ Enriched Uranium

\begin{tabular}{|c|c|c|c|c||}
\hline H/U Ratio & Carbon & Hydrogen & Uranium - 235 & Fluorine \\
\hline 570.6 & 0.03702 & 0.07404 & 0.00013 & 0.00052 \\
\hline 186.4 & 0.03627 & 0.07254 & 0.00039 & 0.00156 \\
\hline 109.5 & 0.03552 & 0.07105 & 0.00065 & 0.0026 \\
\hline 76.6 & 0.03478 & 0.06955 & 0.00091 & 0.00363 \\
\hline 58.3 & 0.03403 & 0.06806 & 0.00117 & 0.00467 \\
\hline 51.9 & 0.03366 & 0.06731 & 0.0013 & 0.00519 \\
\hline 46.6 & 0.03328 & 0.06656 & 0.00143 & 0.00571 \\
\hline 23.1 & 0.02992 & 0.05983 & 0.0026 & 0.01038 \\
\hline 13.4 & 0.02618 & 0.05235 & 0.00389 & 0.01557 \\
\hline 8.6 & 0.02244 & 0.04487 & 0.00519 & 0.02076 \\
\hline 5.8 & 0.0187 & 0.03739 & 0.00649 & 0.02595 \\
\hline 3.8 & 0.01496 & 0.02992 & 0.00779 & 0.03114 \\
\hline 2.5 & 0.01122 & 0.02244 & 0.00908 & 0.03633 \\
\hline 1.4 & 0.00748 & 0.01496 & 0.01038 & 0.04152 \\
\hline 0.64 & 0.00374 & 0.00748 & 0.01168 & 0.04671 \\
\hline
\end{tabular}


POEF-SH-30

Table 3.2-2. Number Densities (atoms/barn-cm) for $\mathbf{U F}_{4}+$ Oil for $10 \%$ Enriched Uranium

\begin{tabular}{||c|c|c|c|c|c||}
\hline B/U Ratio & Carbon & Hydrogen & U-235 & U-238 & Fluorine \\
\hline 576.1 & .03702 & .07404 & .0000129 & .000116 & .000514 \\
\hline 188.2 & .036272 & .072544 & .0000386 & .000347 & .001542 \\
\hline 110.6 & .035524 & .071048 & .0000643 & .000578 & .00257 \\
\hline 77.3 & .034776 & .069553 & .00009 & .00081 & .003598 \\
\hline 71.8 & .034589 & .069179 & .0000964 & .000867 & .003855 \\
\hline 66.9 & .034402 & .068805 & .000103 & .000925 & .004112 \\
\hline 62.6 & .034215 & .068431 & .000109 & .000983 & .004369 \\
\hline 58.8 & .034028 & .068057 & .000116 & .001041 & .004627 \\
\hline 52.4 & .033655 & .067309 & .000129 & .001157 & .005141 \\
\hline 47.1 & .033281 & .066561 & .000141 & .001272 & .005655 \\
\hline 23.3 & .029915 & .05983 & .000257 & .002313 & .010281 \\
\hline 13.6 & .026176 & .052351 & .000386 & .00347 & .015422 \\
\hline 8.7 & .022436 & .044873 & .000514 & .004627 & .020562 \\
\hline 5.8 & .018697 & .037394 & .000643 & .005783 & .025703 \\
\hline 3.9 & .014958 & .029915 & .000771 & .00694 & .030843 \\
\hline 2.5 & .011218 & .022436 & .0009 & .008096 & .035984 \\
\hline 1.5 & .007479 & .014958 & .001028 & .009253 & .041125 \\
\hline 0.64 & .003739 & .007479 & .001157 & .01041 & .046265 \\
\hline
\end{tabular}




\subsection{RESULTS}

\subsection{Supporting Analysis}

The file designation system and a summary of the acronyms used to annotate each of the geometry models is summarized in Table 4.1-1. File names, material type, and geometry models are provided for each of the cases presented in Section 4.0. Keno results are presented in tabular form with the following abbreviations: " $\mathrm{K}$ ssig" is used to mean one sigma on the calculated value of $k$-effective and " $2 * \mathrm{~K}_{-}$sig" is used for twice the sigma. A detailed description of each geometry model is found in Section 2.0.

Table 4.1-1. Geometry Model Summary

\begin{tabular}{|c|l|}
\hline $\begin{array}{c}\text { Geometry Model } \\
\text { Designator }\end{array}$ & \multicolumn{1}{|c|}{ Description of the Model } \\
\hline \hline SFR & Single container full reflection \\
\hline SCC & $\begin{array}{l}\text { Single container in a concrete corner reflected by } 100 \mathrm{~kg} \text { of } \\
\text { water }\end{array}$ \\
\hline SMRC & $\begin{array}{l}\text { Single container with more reactive contents and } 1 \text { inch of } \\
\text { reflection }\end{array}$ \\
\hline TACW & $\begin{array}{l}\text { Two containers touching and adjacent to a concrete wall with } \\
100 \text { kg water reflection in contact }\end{array}$ \\
\hline TSCW & $\begin{array}{l}\text { Two containers touching, 1 foot from concrete wall with } \\
100 \text { kg water reflection in contact }\end{array}$ \\
\hline ACE & Standard array case of double rows \\
\hline AI & Standard array with interstitial moderation \\
\hline AOSP & Standard array with one overloaded storage position \\
\hline
\end{tabular}


POEF-SH-30

\subsubsection{Use of Barber Formula for $\mathrm{H} / \mathrm{U}$ Less Than 4.0}

The use of the Barber formula (Ref. 9) for $\mathrm{H} / \mathrm{U}$ values less than 4.0 results in a peak $k_{\text {eff }}$ at an $\mathrm{H} / \mathrm{U}$ of 3.99. These peak values are conservative but are not considered entirely reasonable from a physical standpoint as discussed in Section 3.2.1. More continuous results are obtained by fixing the uranium density to 3.2 $\mathrm{gU} / \mathrm{cc}$ for $\mathrm{H} / \mathrm{U}$ values less than 4.0 .

All standard contingencies were initially evaluated using the Barber formula. Four 5.0-inch-diameter container contingencies were selected to be reevaluated using the constant uranium density of $3.2 \mathrm{gU} / \mathrm{cc}$. The case selection criterion was based on the results of each case using the Barber formula. Cases that produced $k_{\text {eff }}+2 \sigma$ values of $>0.95$ for $\mathrm{H} / \mathrm{U}$ values less than but not greater than 4.0 using the Barber formula make up the 4 cases reevaluated. All 10\% enriched cases were evaluated using the Barber formula and produced $\mathrm{k}_{\text {eff }}$ values within the criterion.

\subsubsection{Container Thickness Study}

A container thickness study was performed to determine the effect of polyethylene bottle thickness on $k_{\text {eff. }}$. The actual maximum thickness is 0.195 inches based on plant drawings (Ref. 3). The SFR and the TSCW models were evaluated for both the $\mathrm{UO}_{2} \mathrm{~F}_{2}+\mathrm{H}_{2} \mathrm{O}$ and the $\mathrm{UF}_{4}+$ oil cases. Containers modeled adjacent to a wall were not used, because the container thickness would not add significantly to the total reflection of the system.

The results indicate that, for the full reflection model, no significant change in reactivity is noted; however, the two-container case shows a statistically significant increase in reactivity as container thickness increases. For this reason, the two standard reflection models that have containers offset from the concrete walls incorporate the maximum bottle thickness of 0.195 inches. Table 4.1.2-0 illustrates the container thickness results summary. 
POEF-SH-30

Table 4.1.2-0. Container Thickness Result Summary

\begin{tabular}{||c|c|c||}
\hline $\begin{array}{c}\text { Geometry } \\
\text { Model }\end{array}$ & Material & Figure/Table Number \\
\hline \hline SFR & $\mathrm{UO}_{2} \mathrm{~F}_{2}+\mathrm{H}_{2} \mathrm{O}$ & $4.1 .2-1$ \\
\hline TSCW & $\mathrm{UO}_{2} \mathrm{~F}_{2}+\mathrm{H}_{2} \mathrm{O}$ & $4.1 .2-2$ \\
\hline SFR & $\mathrm{UF}_{4}+$ oil & $4.1 .2-3$ \\
\hline TSCW & $\mathrm{UF}_{4}+$ oil & $4.1 .2-4$ \\
\hline
\end{tabular}

The figures and tables listed in Table 4.1.2-0 follow Section 4.2.

\subsubsection{Random Number Generation}

The KENO Va code has an option to change the random number that the run starts. This investigation shows that, depending on the initial number selected, the output can vary by as much as $3 \sigma$. This phenomenon is one possible explanation of the variance that is noted between KENO runs using basically the same input parameters. This range of variance is compensated for by the criteria for a safe condition, which is defined as $\mathrm{k}_{\mathrm{eff}}+2 \sigma<0.95$. The full reflection model was used for this evaluation.

\subsubsection{GP Container Model Assumptions}

GP containers are modeled as $5.0^{\prime \prime} \times 24^{\prime \prime}$ polyethylene cylinders. The actual cylinders have two hollow handle mounts that protrude outside of the 5.0" diameter. Each mount is $1.5^{\prime \prime} \times 1.5^{\prime \prime} \times 1$ "-deep (Ref. 3). They are positioned 180 degrees apart, 20-inches from the base of the container. The calculated volume for each mount assuming no polyethylene thickness is $2.25 \mathrm{in}^{3}$. Two comparison cases were analyzed to show that a simplified model (i.e., no mounts) is adequate for the small container analysis. Both cases model a 5.0" $\times 24^{\prime \prime}$ container filled with $\mathrm{UO}_{2} \mathrm{~F}_{2}$ $+\mathrm{H}_{2} \mathrm{O}$ under full reflection conditions. One case adds a $1.5^{\prime \prime} \times 1.5^{\prime \prime} \times 1.0^{\prime \prime}-$ deep 
Figure 4.1.2-1. Thickness Study for a Single Container of $\mathrm{UO}_{2} \mathrm{~F}_{2}$

Fully Roflected Geometry, 100\% onrichmont, 24" concroto foor, 12" (minimum) water roflector on all other sidos

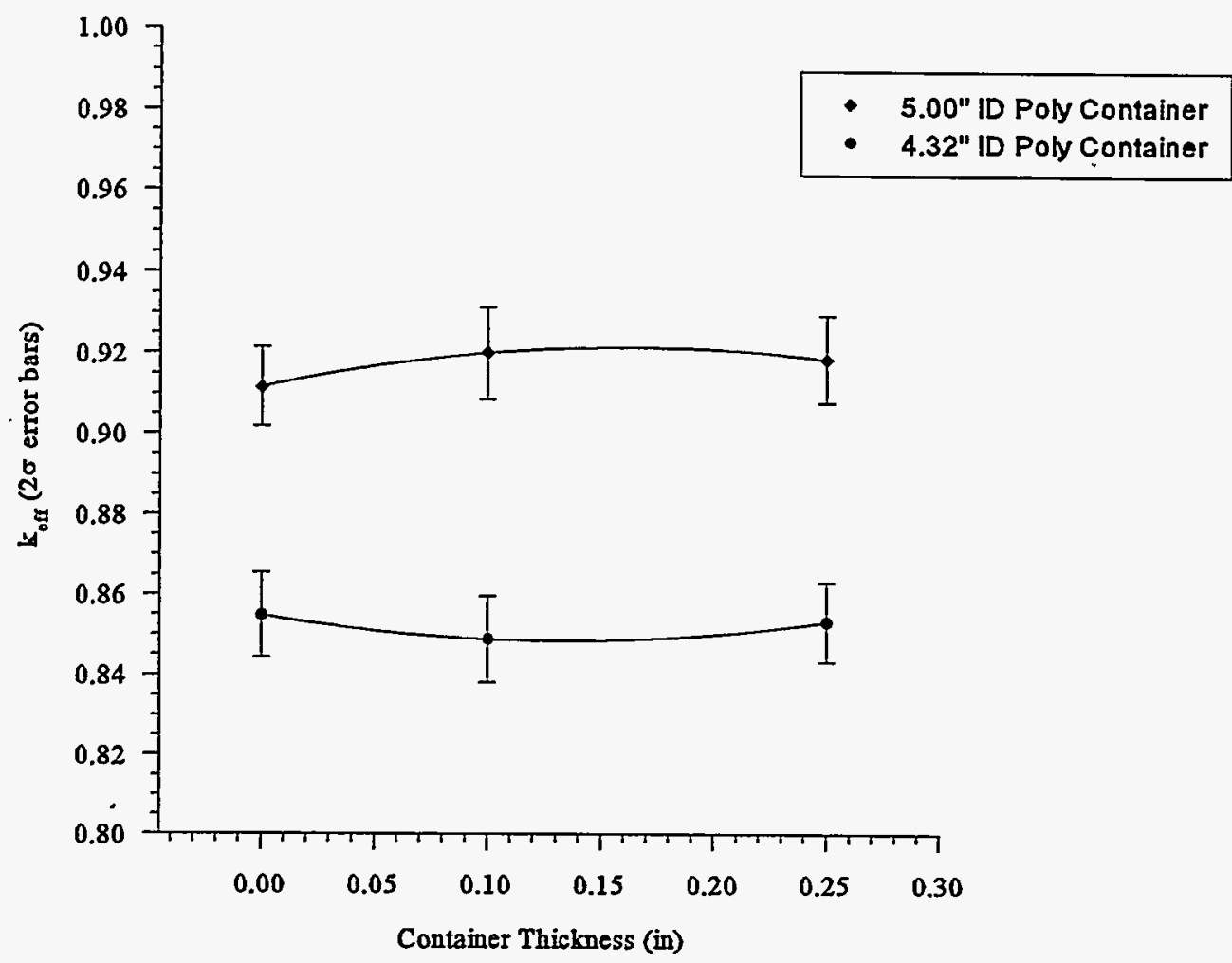

\begin{tabular}{|c|c|c|c|c|c|}
\hline \multicolumn{6}{|c|}{ Single Container Thickness Study for $\mathrm{UO} 2 \mathrm{~F} 2+\mathrm{H} 2 \mathrm{O}$} \\
\hline \multicolumn{6}{|c|}{ with $100 \%$ Enrichment and Full Reflection } \\
\hline Case & $\mathrm{H} / \mathrm{U}$ & Cont Thick & Keff & K_sig & $2^{*} \mathrm{~K}$ sig \\
\hline & & & & & \\
\hline fra011n.o & 25 & 0 & 0.8548 & 0.0053 & 0.0106 \\
\hline fra-c1n.o & 25 & 0.1 & 0.8488 & 0.0054 & 0.0108 \\
\hline fra-c2n.o & 25 & 0.25 & 0.8532 & 0.005 & 0.01 \\
\hline Case & $H / U$ & Cont Thick & Keff & K_sig & $2^{*} \mathrm{~K}$ sig \\
\hline & & & & & \\
\hline & & & & & \\
\hline frb011n.o & 25 & 0 & 0.9116 & 0.0049 & 0.0098 \\
\hline frb-c1n.o & 25 & 0.1 & 0.92 & 0.0057 & 0.0114 \\
\hline frb-c2n.o & 25 & 0.25 & 0.9185 & 0.0054 & 0.0108 \\
\hline
\end{tabular}


Figure 4.1.2-2. Thickness Study for Two 5.00" ID $\times 24 "$ Tall Cylinders of $\mathrm{UO}_{2} \mathrm{~F}_{2}$ $100 \%$ enrichmont, 24" coneroto Iloor, 12" concroto wall and celling, 12" from Wall, -100 kg water reflector simulating a person

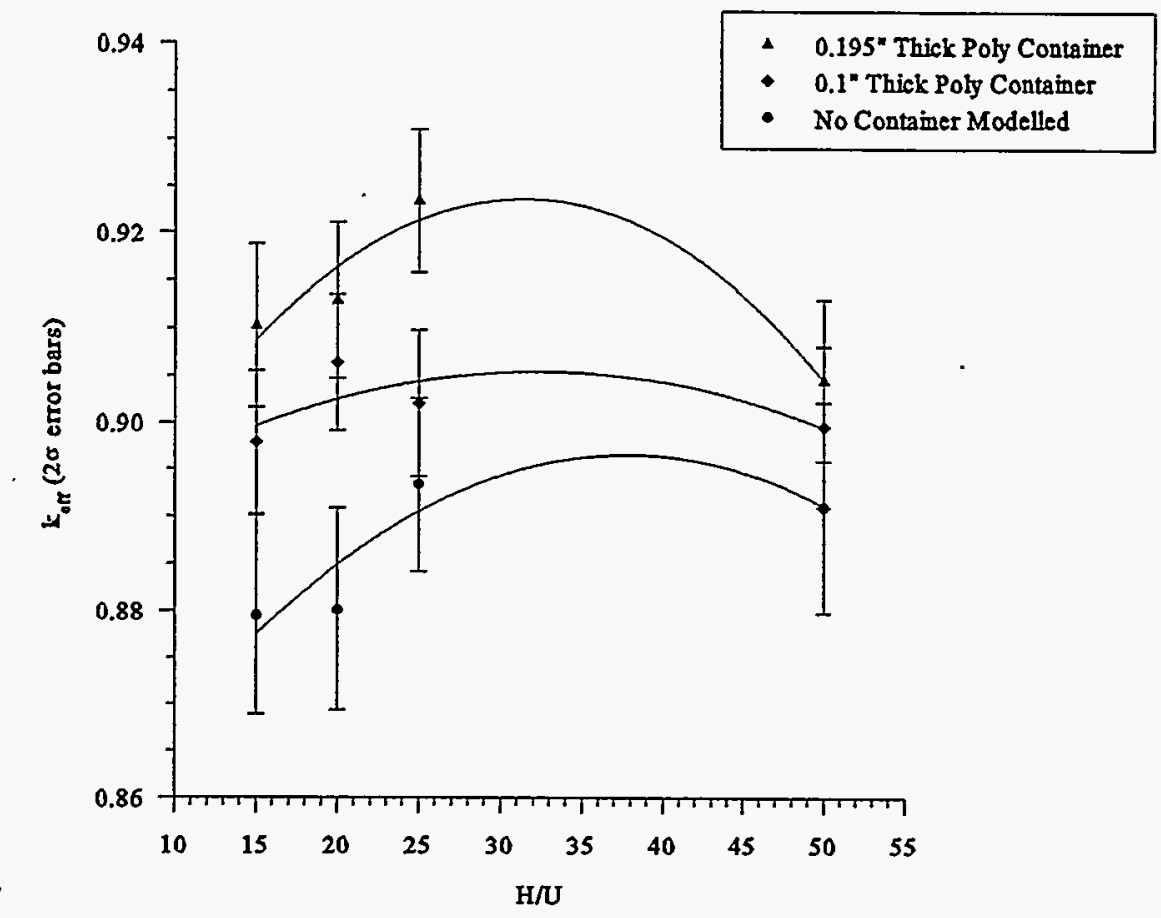

\begin{tabular}{|c|c|c|c|c|}
\hline \multicolumn{5}{|c|}{ Container Thickness Study for 5.0 in. Containers } \\
\hline \multicolumn{5}{|c|}{ of UO2F2+H2O with Full Reflection, $100 \%$ Enrichment } \\
\hline \multicolumn{5}{|l|}{0.195 " Cont } \\
\hline $\mathrm{H} / \mathrm{U}$ & Case & Keff & K_sig & 2*K sig \\
\hline 15 & apb05n-c.o & 0.9102 & 0.0043 & 0.0086 \\
\hline 20 & apbo6n-c.o & 0.9129 & 0.0041 & 0.0082 \\
\hline 25 & apb07n-c.o & 0.9234 & 0.0038 & 0.0076 \\
\hline 50 & apb08n-c.o & 0.9045 & 0.0043 & 0.0086 \\
\hline \multicolumn{5}{|l|}{$0.10^{\prime \prime}$ Cont } \\
\hline $\mathrm{H} / \mathrm{U}$ & Case & Keff & K sig & 2*K sig \\
\hline 15 & apb01n-c.o & 0.8979 & 0.0038 & 0.0076 \\
\hline 20 & apbo2n-c.o & 0.9064 & 0.0036 & 0.0072 \\
\hline 25 & apb03n-c.o & 0.902 & 0.0039 & 0.0078 \\
\hline 50 & apbo $4 n-c .0$ & 0.8996 & 0.0043 & 0.0086 \\
\hline \multicolumn{5}{|l|}{ No Cont } \\
\hline $\mathrm{H} / \mathrm{U}$ & Case & Keff & $K$ sig & $2 * K$ sig \\
\hline 15 & apbo09n.o & 0.8795 & 0.0053 & 0.0106 \\
\hline 20 & apb010n.o & 0.8801 & 0.0054 & 0.0108 \\
\hline 25 & apb011n.o & 0.8934 & 0.0046 & 0.0092 \\
\hline 50 & apb012n.o & 0.891 & 0.0056 & 0.0112 \\
\hline
\end{tabular}


Figure 4.1.2-3. Thickness Study for a Single Container of UF ${ }_{4}$ and Oil

Fully Reflected Geometry, 100\% enrichment, 24" concrete floor, 12" (minimum) water reflector on all other sides

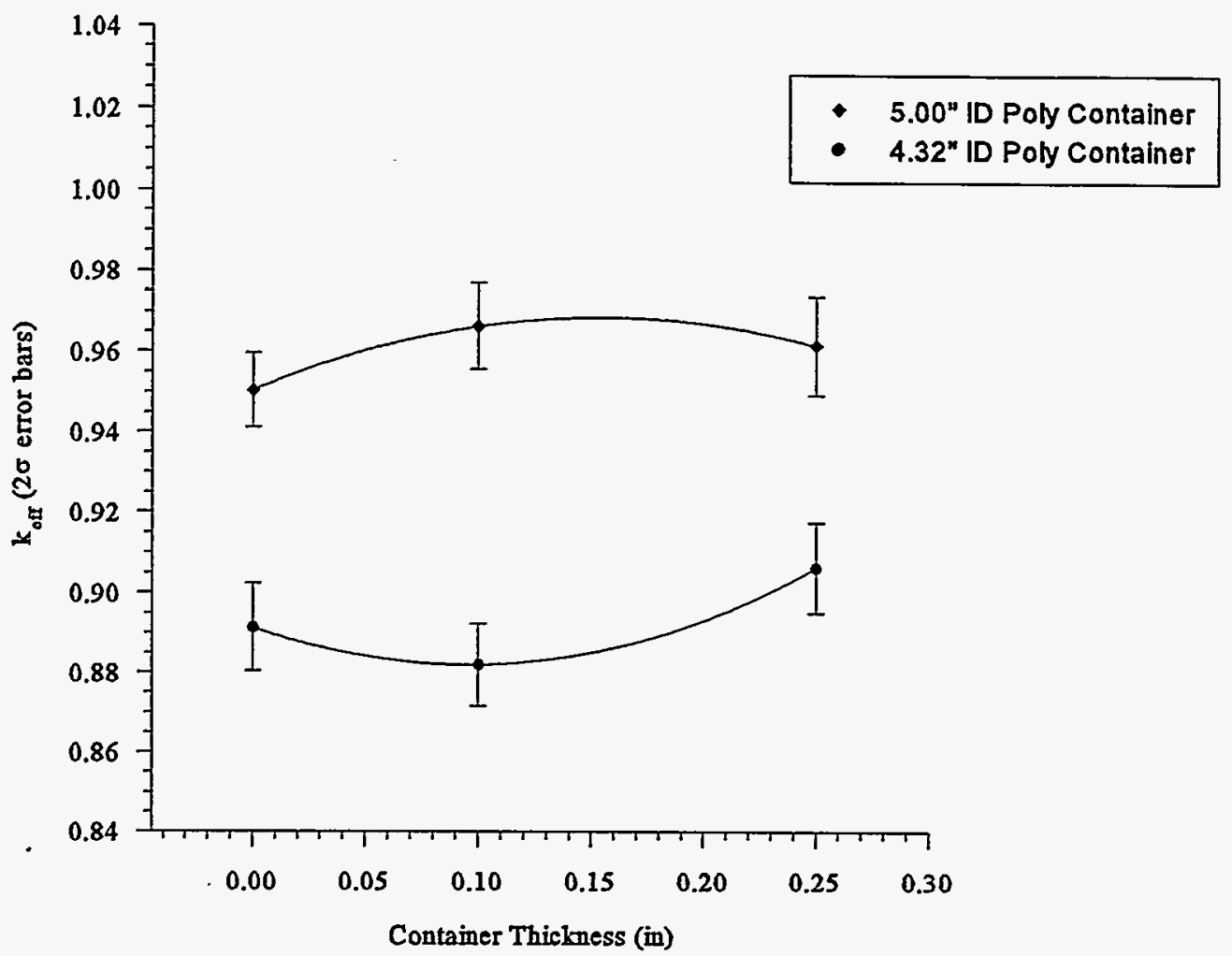

Single Container Study for UF4 and Oil with Full Reflection
\begin{tabular}{|c|c|c|c|c|c|}
\hline \multicolumn{5}{|l|}{ and 100\% Enrichment } \\
\hline & & & & & \\
\hline Case & H/U & Cont Thick & Keff & K_sig & $2^{*}$ K_sig \\
\hline & & & & & \\
\hline & & & & & \\
\hline frd011n.o & 58.2 & 0 & 0.8913 & 0.0055 & 0.011 \\
\hline frd-c1n.o & 58.2 & 0.1 & 0.882 & 0.0052 & 0.0104 \\
\hline frd-c2n.o & 58.2 & 0.25 & 0.9063 & 0.0056 & 0.0112 \\
\hline & & & & & \\
\hline Case & H/U & Cont Thick & Keff & K_sig & $2^{*} \mathrm{~K}$ sig \\
\hline & & & & & \\
\hline & & & & & \\
\hline fre011n.o & 58.2 & 0 & 0.9505 & 0.0046 & 0.0092 \\
\hline fre-c1n.o & 58.2 & 0.1 & 0.9663 & 0.0053 & 0.0106 \\
\hline fre-c2n.o & 58.2 & 0.25 & 0.9616 & 0.0061 & 0.0122 \\
\hline
\end{tabular}


Figure 4.1.2-4. Thickness Study for Two 5.00" ID $\times 24 "$ Tall Cylinders of UF 4 and Oil $100 \%$ enrlehment, 24" concrote Roor, 12" concrete wall and colling, 12" from Wall $-100 \mathrm{~kg}$ wator refloctor simulating a porson

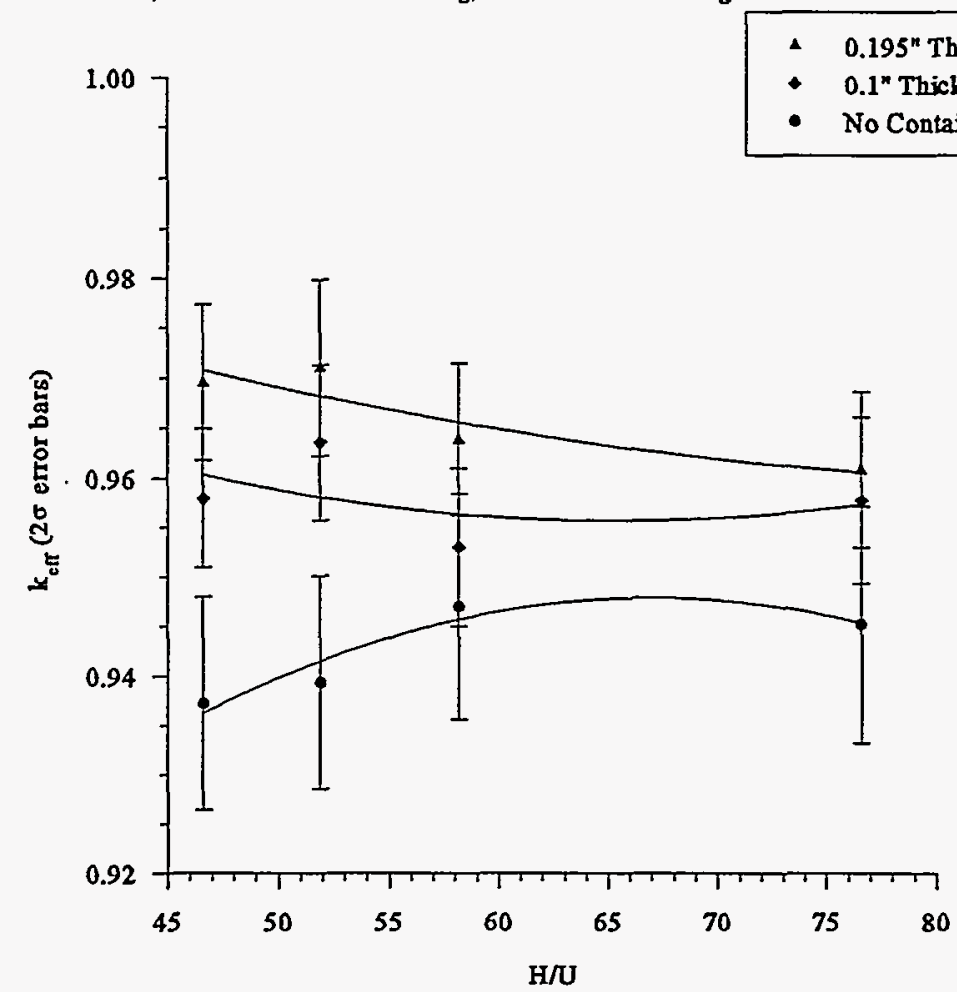

\begin{tabular}{|c|c|c|c|c|}
\hline \multicolumn{5}{|c|}{$1 \mathrm{ft}$. from a Wall for $100 \%$ Enrichment } \\
\hline \multicolumn{5}{|l|}{$0.195 "$ Cont } \\
\hline Case & HIU & Keff & $K$ sig & $2 * K$ sig \\
\hline ape05n-c.o & 46.6 & 0.9695 & 0.0039 & 0.0078 \\
\hline ape06n-c.o & 51.9 & 0.971 & 0.0044 & 0.0088 \\
\hline ape07n-c.o & 58.2 & 0.9638 & 0.0038 & 0.0076 \\
\hline ape08n-c.o & 76.6 & 0.9608 & 0.0039 & 0.0078 \\
\hline \multicolumn{5}{|l|}{0.1 "Cont } \\
\hline Case & H/U & Keff & $K$ sig & $2^{*} \mathrm{~K}$ sig \\
\hline ape01n-c.o & 46.6 & 0.9579 & 0.0035 & 0.007 \\
\hline ape02n-c.o & 51.9 & 0.9635 & 0.0039 & 0.0078 \\
\hline ape03n-c.o & 58.2 & 0.953 & 0.004 & 0.008 \\
\hline ape $04 n-c .0$ & 76.6 & 0.9577 & 0.0042 & 0.0084 \\
\hline \multicolumn{5}{|l|}{ No Cont } \\
\hline Case & $\mathrm{H} / \mathrm{U}$ & Keff & $K$ sig & $2^{*} \mathrm{~K}$ sig \\
\hline ape009n.o & 46.6 & 0.9372 & 0.0054 & 0.0108 \\
\hline ape010n.o & 51.9 & 0.9393 & 0.0054 & 0.0108 \\
\hline ape011n.o & 58.2 & 0.947 & 0.0057 & 0.0114 \\
\hline ape012n.o & 76.6 & 0.9452 & 0.006 & 0.012 \\
\hline
\end{tabular}


POEF-SH-30

ring around the container at a height of 20-inches on the bottle, while in the second case the ring is removed. The results are shown in Table 4.1.4-1. Since the difference in $\mathrm{k}_{\text {eff }}$ is small, and the actual container mounts only occupy $20 \%$ of the total circumference, the simpler model (no mounts) is used throughout this analysis.

Table 4.1.4-1. GP Container Model Assumptions Summary Table

\begin{tabular}{|l|l|c|c|}
\hline \multicolumn{1}{|c|}{ Case } & File Name & H/U & keff + 2sigma \\
\hline $\begin{array}{l}\text { 5" x 24" container with hollow } \\
\text { ring simulating handle mounts } \\
\text { (UO2F2+H2O fully reflected) }\end{array}$ & fr-hand.500 & 20 & 0.9363 \\
\hline Without hollow ring & fr-nohnd.500 & 20 & 0.92244 \\
\hline
\end{tabular}

\subsection{General Dịscussion of Plotted $\mathbf{k}_{\mathrm{erf}}$ versus $\mathbf{H} / \mathbf{U}$ Results}

The $\mathrm{k}_{\text {eff }}$ versus $\mathrm{H} / \mathrm{U}$ plots are generated using SigmaPlot Version 2.0. The curve is a polynomial regression using $\mathrm{k}_{\text {eff }}+2 \sigma$ data points. The $\mathrm{K}_{\mathrm{eff}}$ and $\sigma$ values are generated as part of the output data from the KENO analysis. The error bars represent $\pm 2 \sigma$ for each of the data points. All supporting data, including case identifiers for each of the plots, are presented in tabular form immediately following the plotted results.

The cases employing a constant uranium density of $3.2 \mathrm{gU} / \mathrm{cc}$ for $\mathrm{H} / \mathrm{U}$ values less than 4.0 produce results with a discontinuity at $\mathrm{H} / \mathrm{U}=4.0$. For this reason, an independent polynomial regression is performed for data points below an $\mathrm{H} / \mathrm{U}$ of 4.0 (i.e., the plot is made up of two independent curve fits).

\subsection{32-inch-Diameter by 50-inch Polyethylene Bottle}

This section presents calculations to model the " $744 G$ " and "OG" containers described in Table 1.0-1. 
POEF-SH-30

\subsection{1 $100 \%$ Enriched $\mathrm{UO}_{2} \mathrm{~F}_{2}+\mathrm{H}_{2} \mathrm{O}$ and $\mathrm{UF}_{4}+\mathrm{Oil}$}

Table 4.3.1-0. lists the geometry model, figure, table number, and material type for $100 \%$ enriched 4.32 -inch cases. The geometry model designators are explained in Table 4.1-1. The result of overloading a storage position in an array are shown as data points on the $k_{\text {eff }}$ versus $H / U$ graphs. The plotted curve is through the $k_{\text {eff }}+2 \sigma$ data for the standard array.

Table 4.3.1-0. Summary of 4.32-inch-Diameter Container Results for $100 \%$ Enrichment

\begin{tabular}{|c|c|c||}
\hline Geometry Model & Material & Figure/Table Number \\
\hline \hline SFR & $\mathrm{UO}_{2} \mathrm{~F}_{2}+\mathrm{H}_{2} \mathrm{O}$ & $4.3 .1-1$ \\
\hline SCC & $\mathrm{UO}_{2} \mathrm{~F}_{2}+\mathrm{H}_{2} \mathrm{O}$ & $4.3 .1-2$ \\
\hline TACW & $\mathrm{UO}_{2} \mathrm{~F}_{2}+\mathrm{H}_{2} \mathrm{O}$ & $4.3 .1-3$ \\
\hline TSCW & $\mathrm{UO}_{2} \mathrm{~F}_{2}+\mathrm{H}_{2} \mathrm{O}$ & $4.3 .1-4$ \\
\hline SFR & $\mathrm{UF}_{4}+$ oil & $4.3 .1-5$ \\
\hline SCC & $\mathrm{UF}_{4}+$ oil & $4.3 .1-6$ \\
\hline TACW & $\mathrm{UF}_{4}+$ oil & $4.3 .1-7$ \\
\hline TSCW & $\mathrm{UF}_{4}+$ oil & $4.3 .1-8$ \\
\hline ACE & $\mathrm{UO}_{2} \mathrm{~F}_{2}+\mathrm{H}_{2} \mathrm{O}$ & $4.3 .1-9$ \\
\hline ACE & $\mathrm{UF}_{4}+$ oil & $4.3 .1-10$ \\
\hline AI & $\mathrm{UO}_{2} \mathrm{~F}_{2}+\mathrm{H}_{2} \mathrm{O}$ & $4.3 .1-11$ \\
\hline $\mathrm{AI}$ & $\mathrm{UF}_{4}+\mathrm{oil}$ & $4.3 .1-12$ \\
\hline AOSP & $\mathrm{UO}_{2} \mathrm{~F}_{2}+\mathrm{H}_{2} \mathrm{O}$ & $4.3 .1-13$ \\
\hline AOSP & $\mathrm{UF}_{4}+$ oil & $4.3 .1-14$ \\
\hline
\end{tabular}


Figure 4.3.1-1. Single Fally Reflected 4.32" $\mathrm{I} \times 50^{\prime \prime}$ Tall Cylinder of $\mathrm{UO}{ }_{2} \mathrm{~F}_{2}$ 100\% onfichmont, 24" eoncrete Door, 12" (mIntmum) water roblector on all other obdes

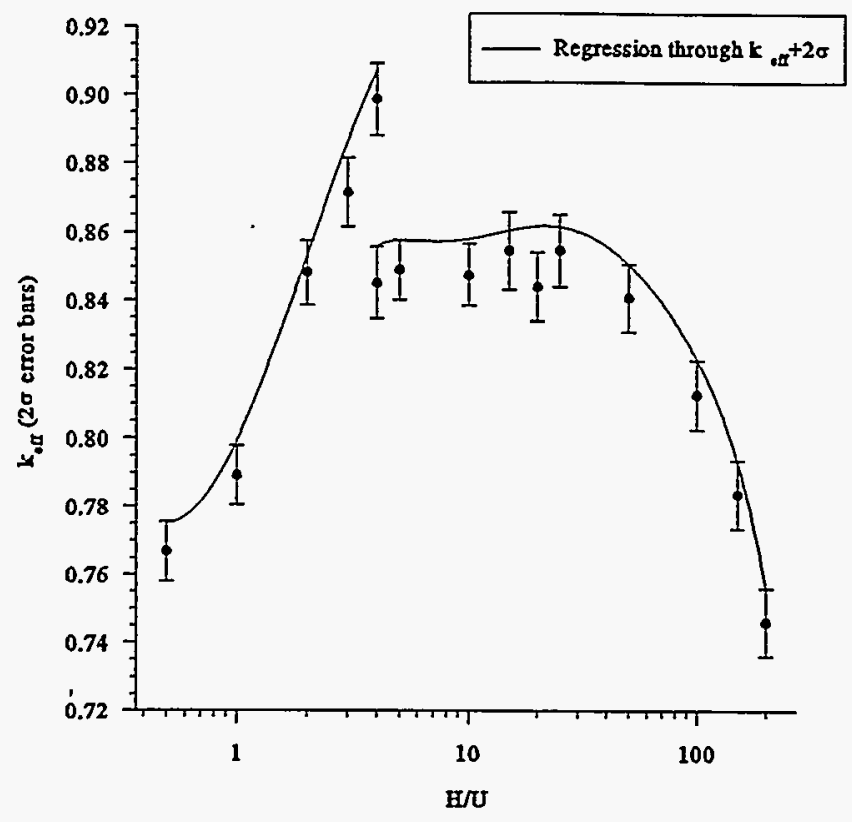

\begin{tabular}{|c|c|c|c|c|c|}
\hline \multicolumn{6}{|c|}{ Single 4.32 in. Diameter Poly. Container w/ Full Reflection } \\
\hline \multicolumn{6}{|c|}{ for $100 \%$ Enriched UO2F $2+\mathrm{H} 2 \mathrm{O}$} \\
\hline & & & & & \\
\hline Case & $H / U$ & Keff & K sig & $2^{\star} \mathrm{K}$ sig & Keff $+2^{*} K$ sig \\
\hline fra001n.o & 0.5 & 0.7668 & 0.0044 & 0.0088 & 0.7756 \\
\hline fra002n.o & 1 & 0.7892 & 0.0043 & 0.0086 & 0.7978 \\
\hline fra003n.o & 2 & 0.8483 & 0.0047 & 0.0094 & 0.8577 \\
\hline fra004n.o & 3 & 0.8716 & 0.005 & 0.01 & 0.8816 \\
\hline fra005n.o & 3.99 & 0.8987 & 0.0053 & 0.0106 & 0.9093 \\
\hline fra006n.o & 4 & 0.8453 & 0.0053 & 0.0106 & 0.8559 \\
\hline fra007n.o & 5 & 0.8491 & 0.0044 & 0.0088 & 0.8579 \\
\hline fra008n.o & 10 & 0.8478 & 0.0045 & 0.009 & 0.8568 \\
\hline fra009n.o & 15 & 0.8548 & 0.0056 & 0.0112 & 0.866 \\
\hline fra010n.o & 20 & 0.8443 & 0.005 & 0.01 & 0.8543 \\
\hline fra011n.o & 25 & 0.8548 & 0.0053 & 0.0106 & 0.8654 \\
\hline fra012n.o & 50 & 0.8411 & 0.0049 & 0.0098 & 0.8509 \\
\hline fra013n.o & 100 & 0.8127 & 0.0051 & 0.0102 & 0.8229 \\
\hline fra014n.o & 150 & 0.7836 & 0.005 & 0.01 & 0.7936 \\
\hline fra015n.o & 200 & 0.746 & 0.0049 & 0.0098 & 0.7558 \\
\hline
\end{tabular}


Figure 4.3.1-2. Single 4.32" ID $\times 50 "$ Tall Cylinder of $U O_{2} F_{2}$ in a Concrete Corner

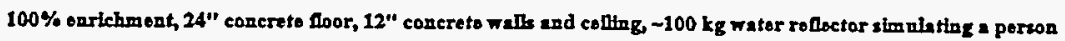

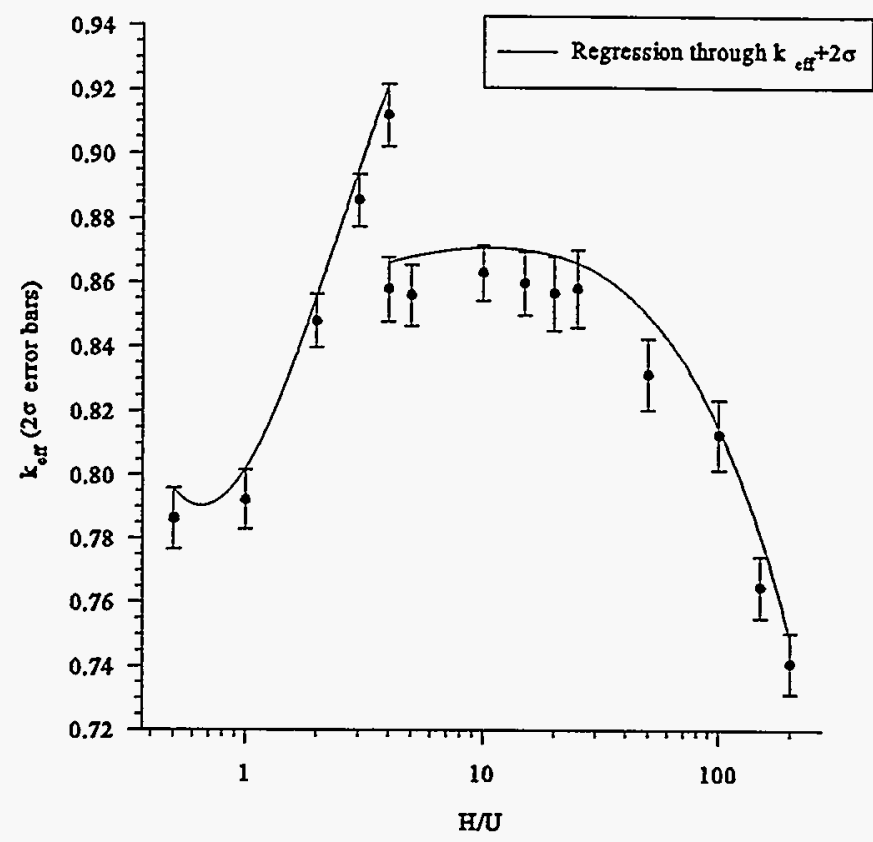

\begin{tabular}{|c|c|c|c|c|c|}
\hline \multicolumn{6}{|c|}{ for $100 \%$ Enriched UO2F2+H2O } \\
\hline Case & $H / U$ & Keff & $K$ sig & $2^{*} \mathrm{~K}$ sig & $K e f f+2^{\star} K \quad$ sia \\
\hline cpa001n.o & 0.5 & 0.7862 & 0.0048 & 0.0096 & 07958 \\
\hline cpa002n.o & 1 & 0.7922 & 0.0047 & 0.0094 & 0.8016 \\
\hline cpa003n.o & 2 & 0.8481 & 0.0042 & 0.0084 & 0.8565 \\
\hline cpa004n.o & 3 & 0.8857 & 0.004 & 0.008 & 0.8937 \\
\hline cpa005n.o & 3.99 & 0.912 & 0.0049 & 0.0098 & 0.9218 \\
\hline cpa006n.o & 4 & 0.8579 & 0.0049 & 0.0098 & 0.8677 \\
\hline cpa007n.o & 5 & 0.8561 & 0.0047 & 0.0094 & 0.8655 \\
\hline cpa008n.o & 10 & 0.8631 & 0.0044 & 0.0088 & 0.8719 \\
\hline cpa009n.o & 15 & 0.8598 & 0.0049 & 0.0098 & 0.8696 \\
\hline cpa010n.o & 20 & 0.8566 & 0.0057 & 0.0114 & 0.868 \\
\hline cpa011n.o & 25 & 0.8581 & 0.006 & 0.012 & 0.8701 \\
\hline cpa012n.o & 50 & 0.8316 & 0.0055 & 0.011 & 0.8426 \\
\hline cpa013n.o & 100 & 0.8127 & 0.0055 & 0.011 & 0.8237 \\
\hline cpa014n.o & 150 & 0.7648 & 0.0048 & 0.0096 & 0.7744 \\
\hline cpa015n.o & 200 & 0.741 & 0.0048 & 0.0096 & 0.7506 \\
\hline
\end{tabular}


Figure 4.3.1-3. Two 4.32" $\mathrm{D} \times 50$ " Tall Cylinders of $\mathrm{UO}{ }_{2} \mathrm{~F}_{2} \mathrm{Nert}$ to a Wall

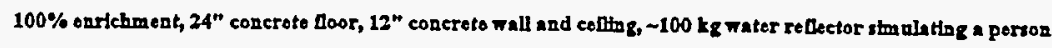

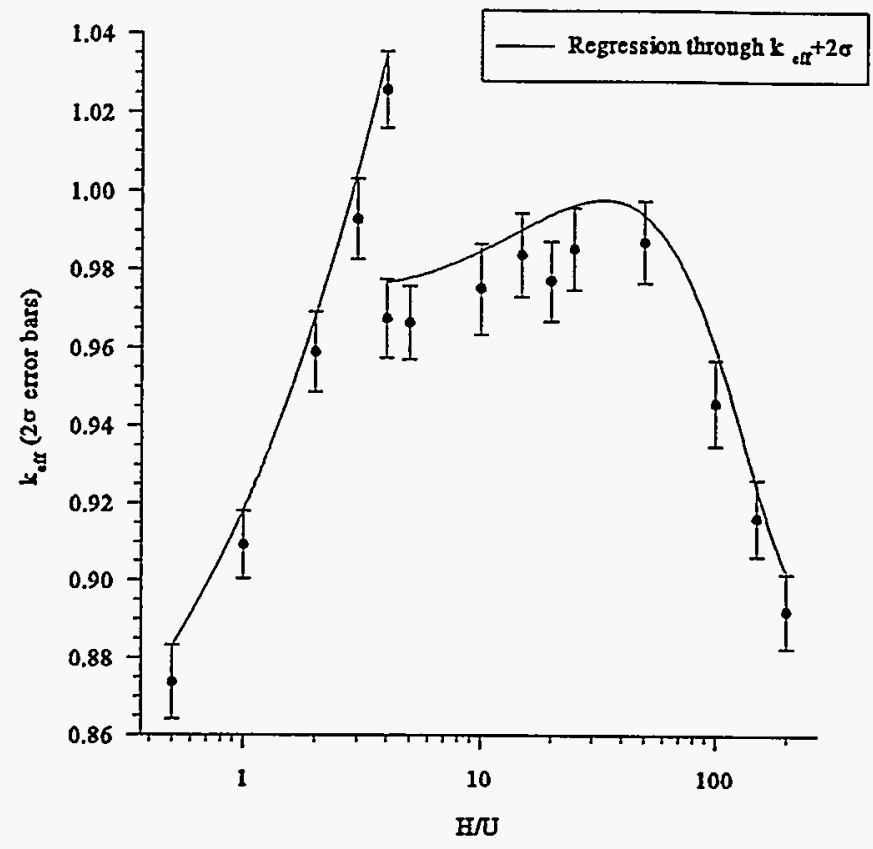

\begin{tabular}{|c|c|c|c|c|c|}
\hline \multicolumn{6}{|c|}{ for $100 \%$ Enriched UO2F2+H2O } \\
\hline Case & $H / U$ & Keff & $\mathrm{K} \mathrm{sia}$ & $2^{*} \mathrm{~K}$ sia & Keff+2*K sia \\
\hline npa001n.o & 0.5 & 08736 & 00048 & 00096 & 08832 \\
\hline npa002n.o & 1 & 0.9093 & 0.0044 & 0.0088 & 0.9181 \\
\hline npa003n.o & 2 & 0.9589 & 0.0051 & 0.0102 & 0.9691 \\
\hline npa004n.o & 3 & 0.9929 & 0.0051 & 0.0102 & 1.0031 \\
\hline npa005n.o & 3.99 & 1.0258 & 0.0049 & 0.0098 & 1.0356 \\
\hline npa006n.o & 4 & 0.9674 & 0.005 & 0.01 & 0.9774 \\
\hline npa007n.o & 5 & 0.9663 & 0.0047 & 0.0094 & 0.9757 \\
\hline npa008n.o & 10 & 0.9752 & 0.0058 & 0.0116 & 0.9868 \\
\hline npa009n.o & 15 & 0.9839 & 0.0054 & 0.0108 & 0.9947 \\
\hline npa010n.o & 20 & 0.9771 & 0.0052 & 0.0104 & 0.9875 \\
\hline npa011n.o & 25 & 0.9854 & 0.0053 & 0.0106 & 0.996 \\
\hline npa012n.o & 50 & 0.9871 & 0.0053 & 0.0106 & 0.9977 \\
\hline npa013n.o & 100 & 0.9459 & 0.0055 & 0.011 & 0.9569 \\
\hline npa014n.o & 150 & 0.9163 & 0.005 & 0.01 & 0.9263 \\
\hline npa015n.o & 200 & 0.8921 & 0.0048 & 0.0096 & 0.9017 \\
\hline
\end{tabular}


Figure 4.3.1-4. Two 4.32" ID $x 50$ " Tall Cylinders of UO ${ }_{2} \mathrm{~F}_{2}$ One Foot from a Wall

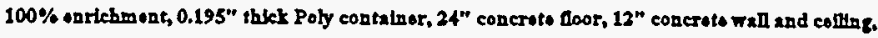
$-100 \mathrm{~kg}$ water rellector stanulattag a perzon

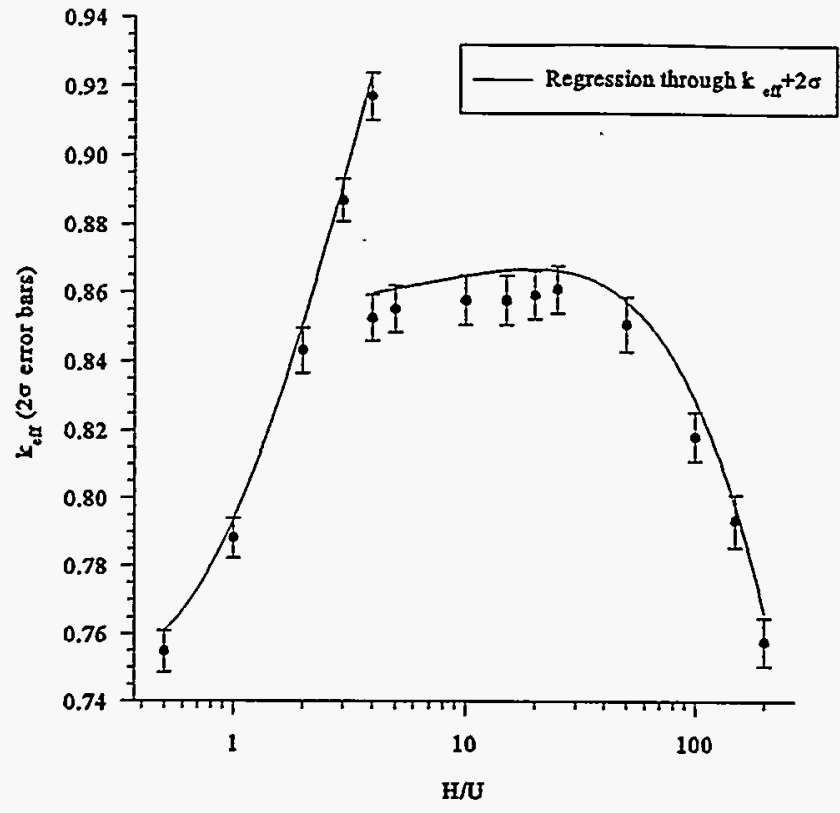

\begin{tabular}{|c|c|c|c|c|c|}
\hline \multicolumn{6}{|c|}{ for $100 \%$ Enriched UO2F $2+\mathrm{H} 2 \mathrm{O}$} \\
\hline & & & & & \\
\hline $\mathrm{H} / \mathrm{U}$ & Case & Keff & K_sig & $2^{\star} \mathrm{K}$ sig & Keff $+2^{\star} K$ sig \\
\hline 0.5 & apa01n-c.o & 0.7548 & 0.0031 & 0.0062 & 0.761 \\
\hline 1 & apa02n-c.o & 0.7881 & 0.0029 & 0.0058 & 0.7939 \\
\hline 2 & apa03n-c.o & 0.8431 & 0.0033 & 0.0066 & 0.8497 \\
\hline 3 & apa04n-c.o & 0.8871 & 0.0031 & 0.0062 & 0.8933 \\
\hline 3.99 & apa05n-c.o & 0.917 & 0.0035 & 0.007 & 0.924 \\
\hline 4 & apa06n-c.o & 0.8526 & 0.0033 & 0.0066 & 0.8592 \\
\hline 5 & apa07n-c.o & 0.8552 & 0.0034 & 0.0068 & 0.862 \\
\hline 10 & apa08n-c.o & 0.8578 & 0.0036 & 0.0072 & 0.865 \\
\hline 15 & apa09n-c.o & 0.8578 & 0.0036 & 0.0072 & 0.865 \\
\hline 20 & apa10n-c.o & 0.8593 & 0.0035 & 0.007 & 0.8663 \\
\hline 25 & apa11n-c.o & 0.861 & 0.0034 & 0.0068 & 0.8678 \\
\hline 50 & apa12n-c.o & 0.8509 & 0.004 & 0.008 & 0.8589 \\
\hline 100 & apa13n-c.o & 0.8181 & 0.0036 & 0.0072 & 0.8253 \\
\hline 150 & apa14n-c.o & 0.7934 & 0.0039 & 0.0078 & 0.8012 \\
\hline 200 & apa15n-c.o & 0.7577 & 0.0036 & 0.0072 & 0.7649 \\
\hline
\end{tabular}


Figure 4.3.1-5. Single Fully Reflected 4.32" ID x 50" Tall Cylinder of UF 4 and Oil $100 \%$ onrichment, 24" concreto floor, 12 " ( $\mathrm{m}$ lntmum) water roflector on all other sides

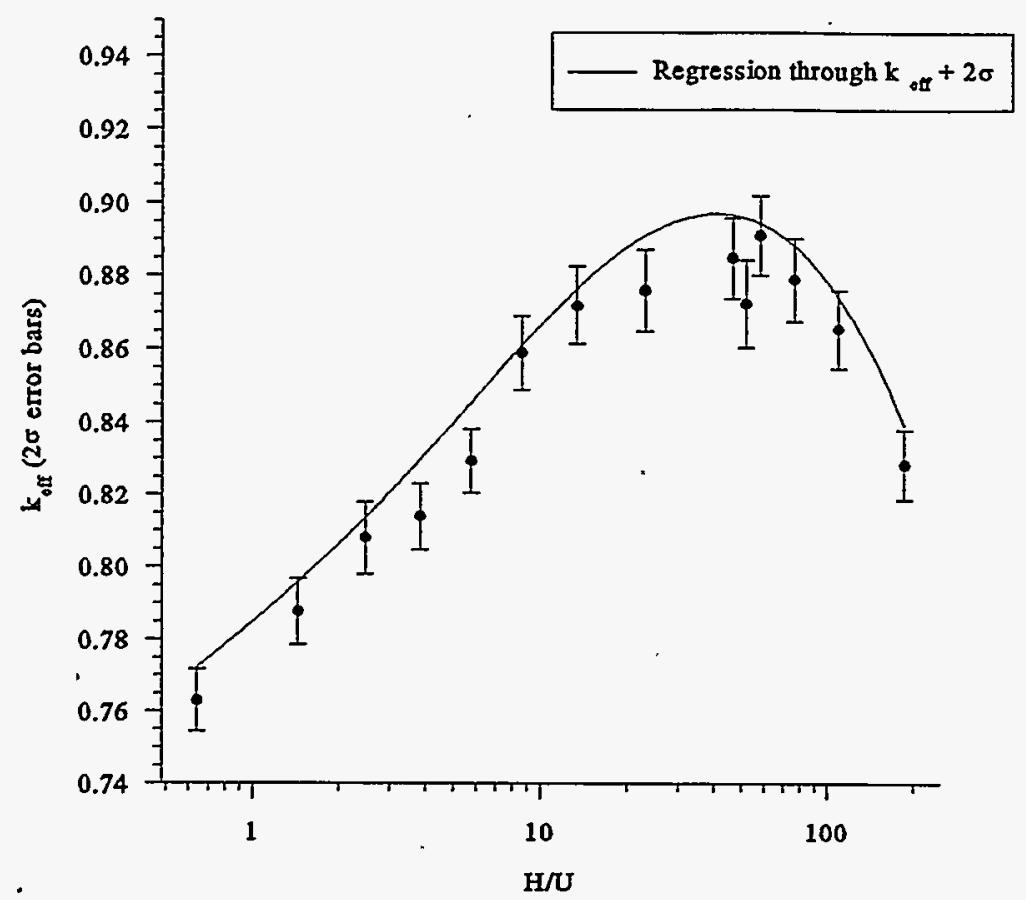

\begin{tabular}{|c|c|c|c|c|c|}
\hline Single 4. & Diam & Contai & rw/ Full & ction & \\
\hline for $100 \%$ & ched UF & & & & \\
\hline & & & & & \\
\hline Case & $\mathrm{H} / \mathrm{U}$ & Keff & $\mathrm{K}$ sig & $2^{*} \mathrm{~K}$ sig & Keff+2*K_sig \\
\hline frd001n.o & 0.64 & 0.763 & 0.0043 & 0.0086 & 0.7716 \\
\hline frd002n.o & 1.44 & 0.7877 & 0.0045 & 0.009 & 0.7967 \\
\hline frd003n.o & 2.47 & 0.808 & 0.005 & 0.01 & 0.818 \\
\hline frd004n.o & 3.84 & 0.814 & 0.0046 & 0.0092 & 0.8232 \\
\hline frdo05n.o & 5.76 & 0.8293 & 0.0044 & 0.0088 & 0.8381 \\
\hline frd006n.o & 8.64 & 0.859 & 0.0051 & 0.0102 & 0.8692 \\
\hline frd007n.o & 13.4 & 0.872 & 0.0053 & 0.0106 & 0.8826 \\
\hline frd008n.o & 23.1 & 0.8761 & 0.0056 & 0.0112 & 0.8873 \\
\hline frd009n.o & 46.6 & 0.8851 & 0.0055 & 0.011 & 0.8961 \\
\hline frd010n.o & 51.9 & 0.8726 & 0.006 & 0.012 & 0.8846 \\
\hline frd011n.o & 58.2 & 0.8913 & 0.0055 & 0.011 & 0.9023 \\
\hline frdo12n.o & 76.6 & 0.8791 & 0.0057 & 0.0114 & 0.8905 \\
\hline frd013n.o & 109.5 & 0.8656 & 0.0054 & 0.0108 & 0.8764 \\
\hline frd014n.o & 186.4 & 0.8283 & 0.0048 & 0.0096 & 0.8379 \\
\hline
\end{tabular}


Figure 4.3.1-6. Single 4.32" ID x 50" Tall Cylinder of UF ${ }_{4}$ and Oil in a Concrete Corner $100 \%$ on richment, $24^{\prime \prime}$ concrote foor, 12" concreto wall and ceilling, $\sim 100 \mathrm{~kg}$ water reflector stmalating a perton

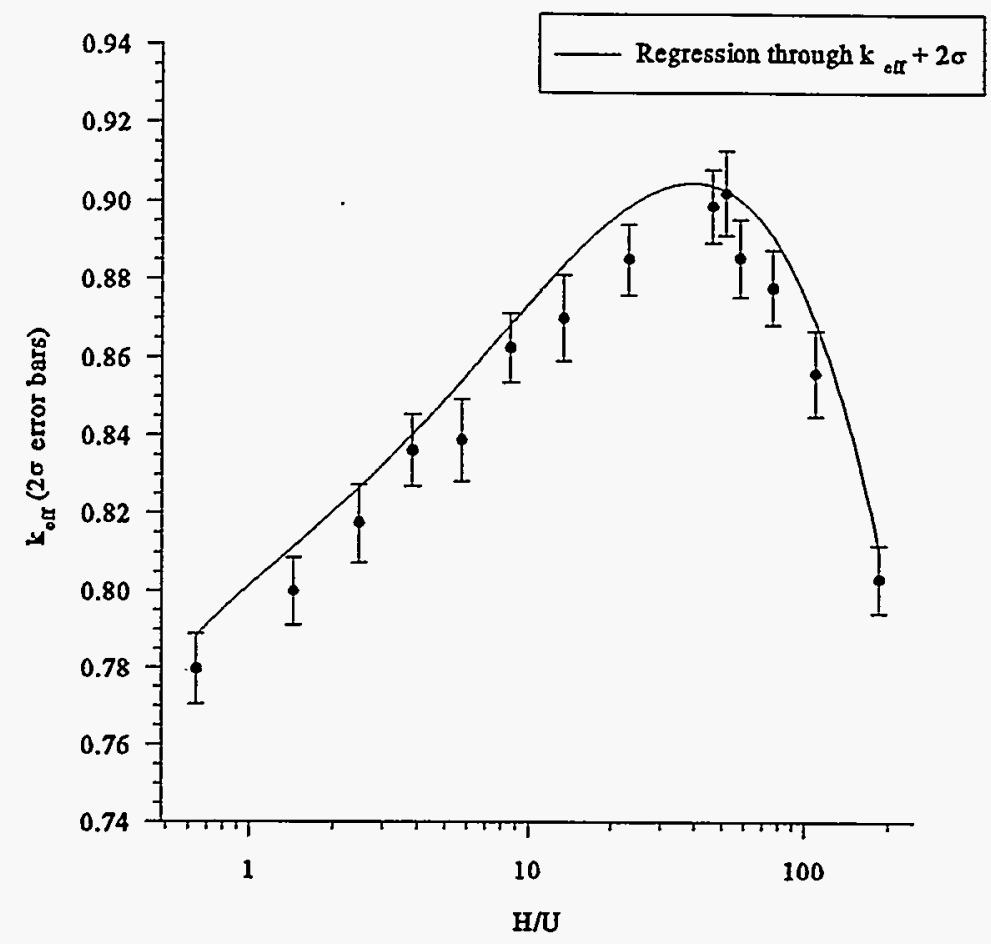

\begin{tabular}{|c|c|c|c|c|c|}
\hline \multicolumn{6}{|c|}{ for $100 \%$ Enriched UF4+oil } \\
\hline & & & & & \\
\hline Case & $H / U$ & Keff & $\mathrm{K}$ sig & $2^{*} \mathrm{~K}$ sig & Keff $+2^{\star} K$ sig \\
\hline cpd001n.o & 0.64 & 0.7797 & 0.0046 & 0.0092 & 0.7889 \\
\hline cpd002n.o & 1.44 & 0.7999 & 0.0044 & 0.0088 & 0.8087 \\
\hline cpd003n.o & 2.47 & 0.8174 & 0.005 & 0.01 & 0.8274 \\
\hline cpd004n.o & 3.84 & 0.8361 & 0.0046 & 0.0092 & 0.8453 \\
\hline cpd005n.o & 5.76 & 0.8387 & 0.0053 & 0.0106 & 0.8493 \\
\hline cpd006n.o & 8.64 & 0.8624 & 0.0044 & 0.0088 & 0.8712 \\
\hline cpd007n.o & 13.4 & 0.87 & 0.0055 & 0.011 & 0.881 \\
\hline cpd008n.o & 23.1 & 0.8851 & 0.0045 & 0.009 & 0.8941 \\
\hline cpd009n.o & 46.6 & 0.8988 & 0.0047 & 0.0094 & 0.9082 \\
\hline cpd010n.o & 51.9 & 0.9021 & 0.0054 & 0.0108 & 0.9129 \\
\hline cpd011n.o & 58.2 & 0.8855 & 0.0049 & 0.0098 & 0.8953 \\
\hline cpd012n.o & 76.6 & 0.8779 & 0.0048 & 0.0096 & 0.8875 \\
\hline cpd013n.o & 109.5 & 0.856 & 0.0055 & 0.011 & 0.867 \\
\hline cpd014n.o & 186.4 & 0.8032 & 0.0044 & 0.0088 & 0.812 \\
\hline
\end{tabular}


Figure 4.3.1-7. Two 4.32" $\mathrm{I} \times 5$ 50" Tall Cylinders of UF $_{4}$ and Oil Next to a Wall $100 \%$ onrichmont, 24 " concrote foor, 12" concrete wall and coiling, $-100 \mathrm{~kg}$ water refoetor simnlating a porson

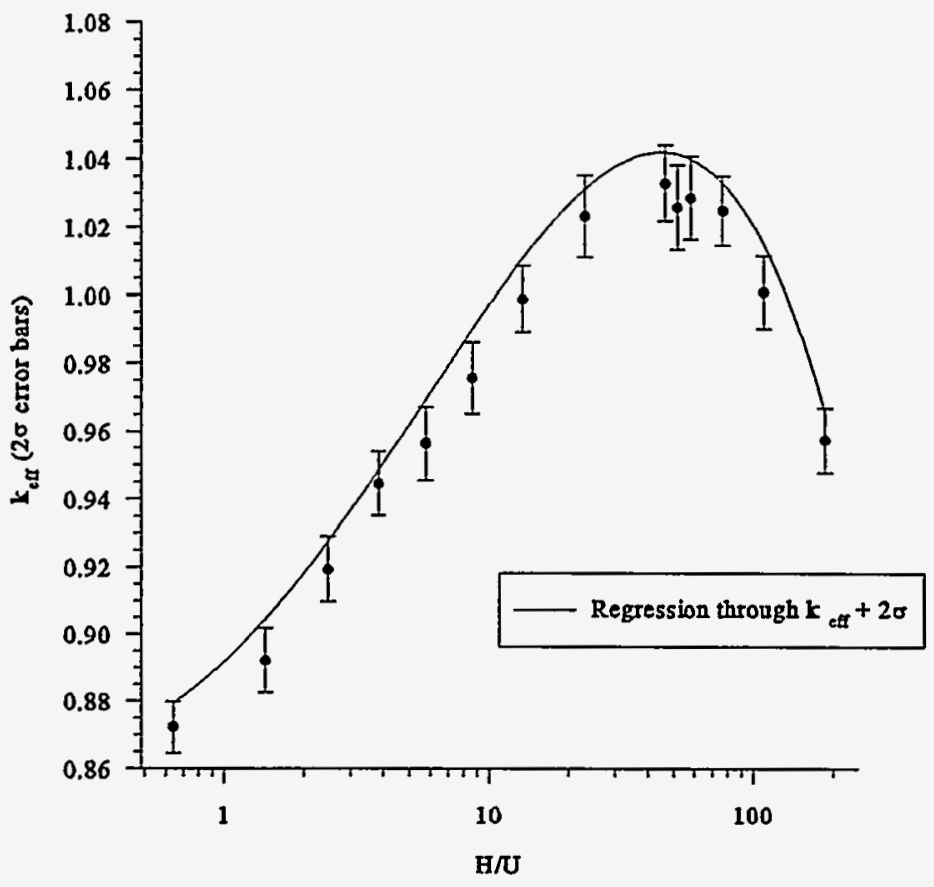

Two 4.32 in. Diameter Poly. Containers Adjacent to Concrete Wall for $100 \%$ Enriched UF4+oil

\begin{tabular}{|c|c|c|c|c|c|}
\hline & & & & & \\
\hline Case & H/U & Keff & K sig & $2^{*}$ K sig & Keff+2*K sig \\
\hline & & & & & \\
\hline npd001n.o & 0.64 & 0.8722 & 0.0038 & 0.0076 & 0.8798 \\
\hline npd002n.o & 1.44 & 0.892 & 0.0048 & 0.0096 & 0.9016 \\
\hline npd003n.o & 2.47 & 0.9192 & 0.0048 & 0.0096 & 0.9288 \\
\hline npd004n.o & 3.84 & 0.9446 & 0.0047 & 0.0094 & 0.954 \\
\hline npd005n.o & 5.76 & 0.9565 & 0.0054 & 0.0108 & 0.9673 \\
\hline npd006n.o & 8.64 & 0.9758 & 0.0052 & 0.0104 & 0.9862 \\
\hline npd007n.o & 13.4 & 0.999 & 0.0049 & 0.0098 & 1.0088 \\
\hline npd008n.o & 23.1 & 1.0234 & 0.0061 & 0.0122 & 1.0356 \\
\hline npd009n.o & 46.6 & 1.0331 & 0.0055 & 0.011 & 1.0441 \\
\hline npd010n.o & 51.9 & 1.026 & 0.0063 & 0.0126 & 1.0386 \\
\hline npd011n.o & 58.2 & 1.0287 & 0.0061 & 0.0122 & 1.0409 \\
\hline npd012n.o & 76.6 & 1.025 & 0.0051 & 0.0102 & 1.0352 \\
\hline npd013n.o & 109.5 & 1.0011 & 0.0054 & 0.0108 & 1.0119 \\
\hline npd014n.o & 186.4 & 0.9574 & 0.0048 & 0.0096 & 0.967 \\
\hline
\end{tabular}


Figure 4.3.1-8. Two 4.32" ID $\times$ 50" Tall Cylinders of UF 4 and Oil One Foot from a Wall $100 \%$ enrichment, $24^{\prime \prime}$ concrete floor, 12 " concrete wall and ceiling, $\sim 100 \mathrm{~kg}$ water reflector simulating a person

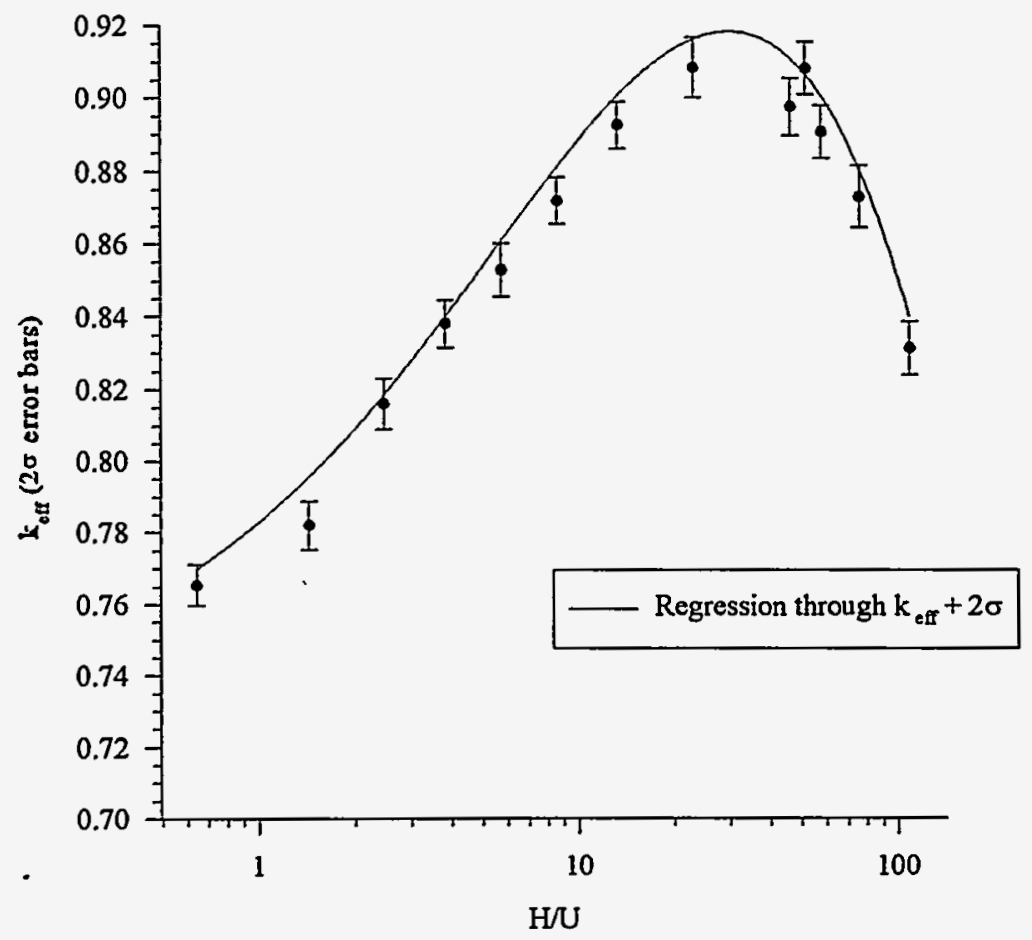

Two 4.32 in. Diameter Poly. Containers 1' from Concrete Wall for $100 \%$ Enriched UF4+oil

\begin{tabular}{|c|c|c|c|c|c|}
\hline \hline Case & H/U & Keff & K sig & $2^{\star}$ K_sig & Keff+2*K_sig \\
\hline d12n-432.0p6 & 0.64 & 0.7655 & 0.00291 & 0.00582 & 0.77132 \\
\hline$d 12 n-432.144$ & 1.44 & 0.782 & 0.00344 & 0.00688 & 0.78888 \\
\hline$d 12 n-432.384$ & 3.84 & 0.816 & 0.0035 & 0.007 & 0.823 \\
\hline$d 12 n-432.576$ & 5.76 & 0.838 & 0.00327 & 0.00654 & 0.84454 \\
\hline$d 12 n-432.864$ & 8.64 & 0.8528 & 0.00364 & 0.00728 & 0.86008 \\
\hline$d 12 n-432.134$ & 13.4 & 0.8718 & 0.00322 & 0.00644 & 0.87824 \\
\hline$d 12 n-432.231$ & 23.1 & 0.8924 & 0.00319 & 0.00638 & 0.89878 \\
\hline$d 12 n-432.466$ & 46.6 & 0.9081 & 0.00418 & 0.00836 & 0.91646 \\
\hline$d 12 n-432.519$ & 51.9 & 0.8974 & 0.00395 & 0.0079 & 0.9053 \\
\hline$d 12 n-432.582$ & 58.2 & 0.908 & 0.00365 & 0.0073 & 0.9153 \\
\hline$d 12 n-432.766$ & 76.6 & 0.8906 & 0.00358 & 0.00716 & 0.89776 \\
\hline$d 12 n-432.109$ & 109.5 & 0.873 & 0.00425 & 0.0085 & 0.8815 \\
\hline$d 12 n-432.186$ & 186.4 & 0.8312 & 0.00367 & 0.00734 & 0.83854 \\
\hline
\end{tabular}


Figure 4.3.1-9. An Array of 4.32" ID $~ 550$ " Tall Cylinders of UO ${ }_{2} F_{2}$

100\% onrichm ont, 0.195" theck Poty contelners, 24" concrote Door, 12" cozerote walle and cellth

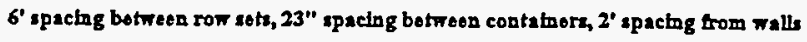

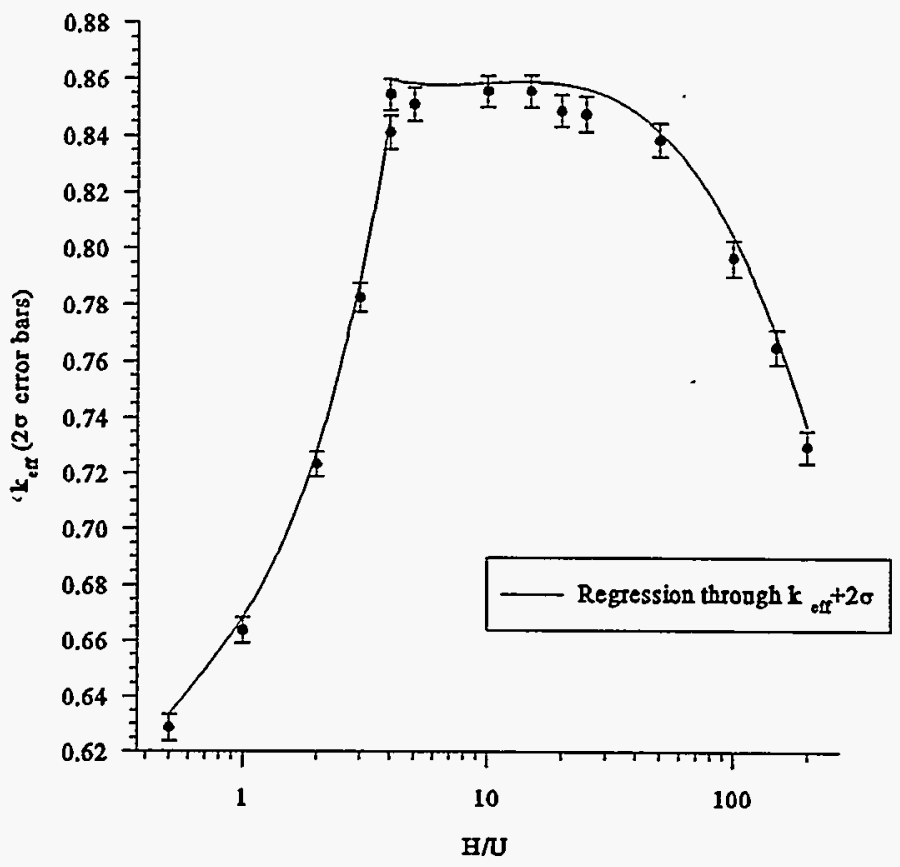

\begin{tabular}{|c|c|c|c|c|c|}
\hline \multicolumn{6}{|c|}{ An Array of 4.32 -in. Containers of $\mathrm{UO} 2 \mathrm{~F} 2$} \\
\hline \multicolumn{6}{|c|}{ For $100 \%$ Enrichment } \\
\hline Case & $\mathrm{H} / \mathrm{U}$ & Keff & K_sig & $2^{*} \mathrm{~K}$ sig & Keff+2*K_sig \\
\hline arra01n-c.o & 0.5 & 0.6285 & 0.0024 & 0.0048 & 0.6333 \\
\hline arra02n-c.o & 1 & 0.6637 & 0.0023 & 0.0046 & 0.6683 \\
\hline arra03n-c.o & 2 & 0.7233 & 0.0023 & 0.0046 & 0.7279 \\
\hline arra04n-c.o & 3 & 0.7827 & 0.0027 & 0.0054 & 0.7881 \\
\hline arra05n-c.o & 3.99 & 0.8413 & 0.0029 & 0.0058 & 0.8471 \\
\hline arra06n-c.o & 4 & 0.8546 & 0.0028 & 0.0056 & 0.8602 \\
\hline arra07n-c.o & 5 & 0.8512 & 0.0029 & 0.0058 & 0.857 \\
\hline arra08n-c.o & 10 & 0.8558 & 0.0028 & 0.0056 & 0.8614 \\
\hline arra09n-c.o & 15 & 0.8559 & 0.0029 & 0.0058 & 0.8617 \\
\hline arra10n-c.o & 20 & 0.849 & 0.0028 & 0.0056 & 0.8546 \\
\hline arra1 1n-c.o & 25 & 0.8479 & 0.0031 & 0.0062 & 0.8541 \\
\hline arra12n-c.o & 50 & 0.8388 & 0.0029 & 0.0058 & 0.8446 \\
\hline arra13n-c.o & 100 & 0.797 & 0.0031 & 0.0062 & 0.8032 \\
\hline arra $14 n-c .0$ & 150 & 0.7653 & 0.003 & 0.006 & 0.7713 \\
\hline arra15n-c.o & 200 & 0.7299 & 0.003 & 0.006 & 0.7359 \\
\hline
\end{tabular}


Figure 4.3.1-10. An Array of 4.32" $\mathrm{D}$ × 50" Tall Cylinders of UF $4+$ Oil

100\% enrichment, 0.195" thlek Poly contahers, 24" coneroto 100r, 12" conereto walle and ceilling,

6' spacing betwoes row sets, 23" spachg betwoen containers, 2' spacing from walb

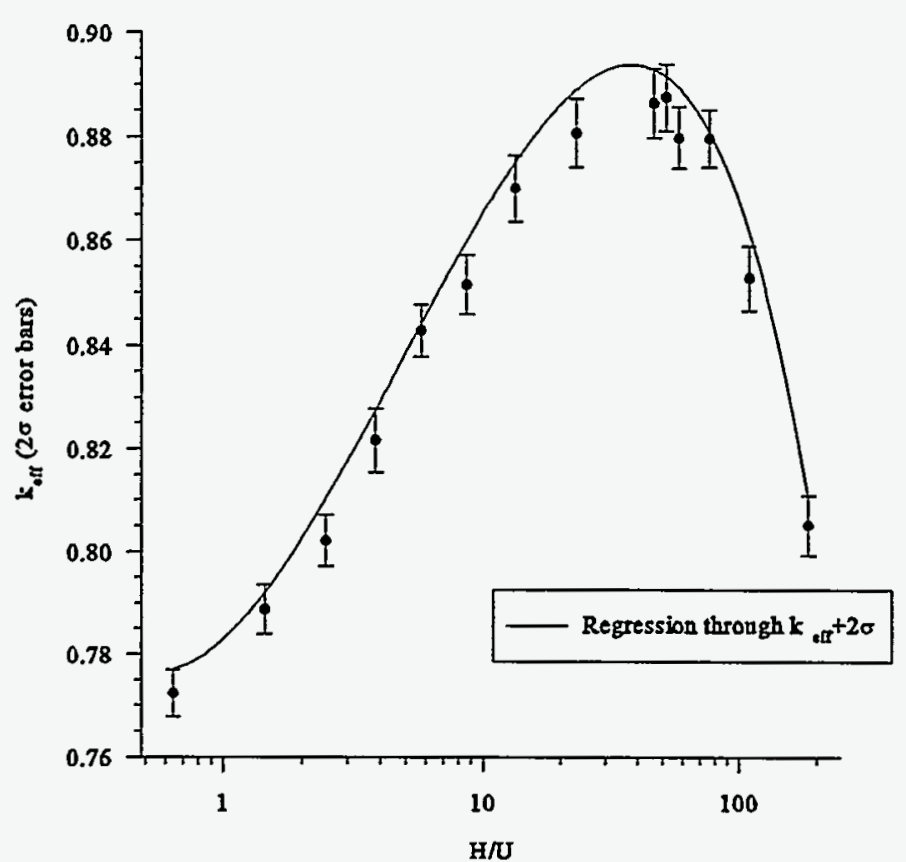

\begin{tabular}{|c|c|c|c|c|c|}
\hline \multicolumn{6}{|l|}{ An Array of 4.32-in. Poly. Containers of UF4+Oil } \\
\hline For 100\% Enrichment & & & & \\
\hline Case & HN & Keff & K sig & $2^{*}$ K_sig & Keff+2* K sig \\
\hline & & & & & \\
\hline arrd01n-c.o & 0.64 & 0.7723 & 0.0023 & 0.0046 & 0.7769 \\
\hline arrd02n-c.o & 1.44 & 0.7887 & 0.0024 & 0.0048 & 0.7935 \\
\hline arrd03n-c.o & 2.47 & 0.8021 & 0.0025 & 0.005 & 0.8071 \\
\hline arrd04n-c.o & 3.84 & 0.8217 & 0.0031 & 0.0062 & 0.8279 \\
\hline arrd05n-c.o & 5.76 & 0.8428 & 0.0025 & 0.005 & 0.8478 \\
\hline arrd06n-c.o & 8.64 & 0.8516 & 0.0028 & 0.0056 & 0.8572 \\
\hline arrd07n-c.o & 13.4 & 0.8701 & 0.0032 & 0.0064 & 0.8765 \\
\hline arrd08n-c.o & 23.1 & 0.8807 & 0.0032 & 0.0064 & 0.8871 \\
\hline arrd09n-c.o & 46.6 & 0.8864 & 0.0033 & 0.0066 & 0.893 \\
\hline arrd10n-c.o & 51.9 & 0.8875 & 0.0032 & 0.0064 & 0.8939 \\
\hline arrd11n-c.o & 58.2 & 0.8798 & 0.0029 & 0.0058 & 0.8856 \\
\hline arrd12n-c.o & 76.6 & 0.8796 & 0.0027 & 0.0054 & 0.885 \\
\hline arrd13n-c.o & 109.5 & 0.8529 & 0.0031 & 0.0062 & 0.8591 \\
\hline arrd14n-c.o & 186.4 & 0.8051 & 0.0029 & 0.0058 & 0.8109 \\
\hline
\end{tabular}


Figure 4.3.1-11. The Effects of Interstitial Water Volume Fraction on $k_{e r r}$ Standard array geometry with 4.32 " ID x 50" tall containers of $U{ }_{2} F_{2}$ with $0.195^{\prime \prime}$ thick Poly walls

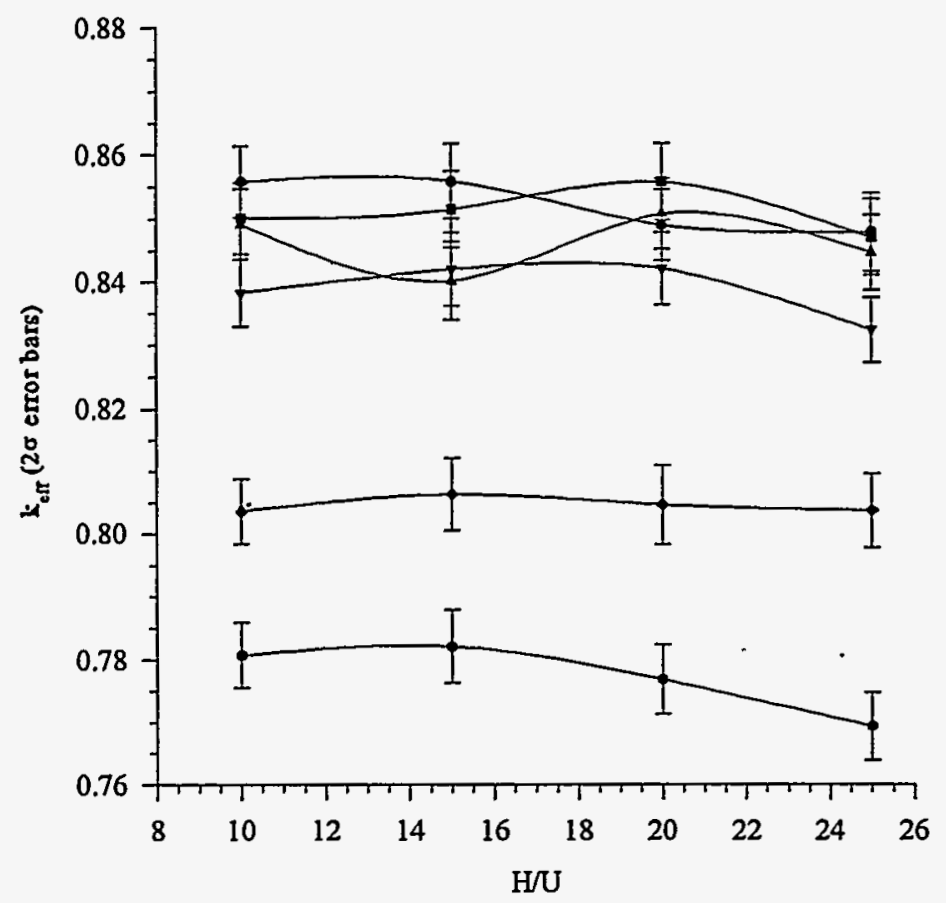

$\longrightarrow$ Water Volume Fraction $=0.0$

$\rightarrow-$ Water Volume Fraction $=0.001$

$\longrightarrow$ Water Volume Fraction $=0.005$

$\rightarrow$ Water Volume Fraction $=0.01$

$\rightarrow$ Water Volume Fraction $=0.05$

$\rightarrow-$ Water Volume Fraction $=0.1$ 
Figure 4.3.1-11a. The Effects of Interstitial Water Volume Fraction on $\mathbf{k}_{\text {eff }}$ Standard array geometry with $4.32 " \mathrm{ID} \times 50^{\prime \prime}$ tall containers of $\mathrm{UO}_{2} \mathrm{~F}_{2}$ with $0.195^{\prime \prime}$ thick Poly walls

\begin{tabular}{|c|c|c|c|c|c|}
\hline \multicolumn{5}{|l|}{ The Effects of interstitial Water Volume Fraction for a Standard } \\
\hline Array of 4.32-in. Diameter Containers of UO2F2+H2O \\
\hline \hline Case & H/U & Keff & K sig & $2^{*}$ K sig & Keff +2*K sig \\
\hline Interstitial & Water $=$ & 0 & & & \\
\hline arra8n-c.o & 10 & 0.8558 & 0.0028 & 0.0056 & 0.8614 \\
\hline arra09n-c.o & 15 & 0.8559 & 0.0029 & 0.0058 & 0.8617 \\
\hline arra10n-c.o & 20 & 0.849 & 0.0028 & 0.0056 & 0.8546 \\
\hline arra11n-c.o & 25 & 0.8479 & 0.0031 & 0.0062 & 0.8541 \\
\hline Interstitial & Water $=$ & 0.001 & & & \\
\hline aaw101n-c.o & 10 & 0.8501 & 0.0028 & 0.0056 & 0.8557 \\
\hline aaw102n-c.o & 15 & 0.8515 & 0.003 & 0.006 & 0.8575 \\
\hline aaw103n-c.o & 20 & 0.8558 & 0.003 & 0.006 & 0.8618 \\
\hline aaw104n-c.o & 25 & 0.8471 & 0.003 & 0.006 & 0.8531 \\
\hline Interstitial & Water $=$ & 0.005 & & & \\
\hline aaw201n-c.o & 10 & 0.8492 & 0.0028 & 0.0056 & 0.8548 \\
\hline aaw202n-c.o & 15 & 0.8402 & 0.0031 & 0.0062 & 0.8464 \\
\hline aaw203n-c.o & 20 & 0.8508 & 0.0028 & 0.0056 & 0.8564 \\
\hline aaw204n-c.o & 25 & 0.8447 & 0.003 & 0.006 & 0.8507 \\
\hline Interstitial & Water $=$ & 0.01 & & & \\
\hline aaw301n-c.o & 10 & 0.8383 & 0.0027 & 0.0054 & 0.8437 \\
\hline aaw302n-c.o & 15 & 0.842 & 0.0029 & 0.0058 & 0.8478 \\
\hline aaw303n-c.o & 20 & 0.8421 & 0.0029 & 0.0058 & 0.8479 \\
\hline aaw304n-c.o & 25 & 0.8324 & 0.0026 & 0.0052 & 0.8376 \\
\hline Interstitial & Water $=$ & 0.05 & & & \\
\hline aaw401n-c.o & 10 & 0.8036 & 0.0026 & 0.0052 & 0.8088 \\
\hline aaw402n-c.o & 15 & 0.8063 & 0.0029 & 0.0058 & 0.8121 \\
\hline aaw403n-c.o & 20 & 0.8046 & 0.0032 & 0.0064 & 0.811 \\
\hline aaw404n-c.o & 25 & 0.8037 & 0.003 & 0.006 & 0.8097 \\
\hline Interstitial & Water $=$ & 0.1 & & & \\
\hline aaw501n-c.o & 10 & 0.7807 & 0.0026 & 0.0052 & 0.7859 \\
\hline aaw502n-c.o & 15 & 0.7821 & 0.0029 & 0.0058 & 0.7879 \\
\hline aaw503n-c.o & 20 & 0.7768 & 0.0028 & 0.0056 & 0.7824 \\
\hline aaw504n-c.o & 25 & 0.7693 & 0.0027 & 0.0054 & 0.7747 \\
\hline
\end{tabular}


Figure 4.3.1-12. The Effects of In terstitial Water Volume Fraction on $k$ efr Standard array geometry with 4.32" ID x 50" tall containers of UP, and Oil with $0.195^{\prime \prime}$ thick Poly walls

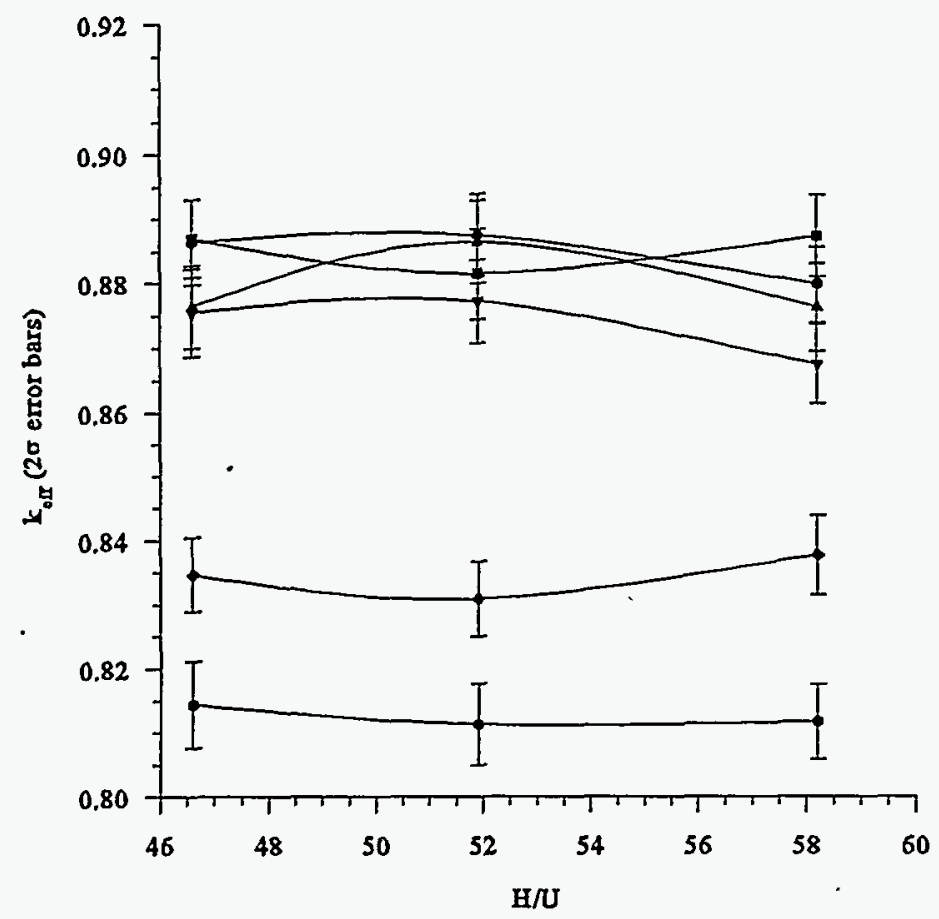


Figure 4.3.1-12a. The Effects of Interstitial Water Volume Fraction on $k_{\text {eff }}$ Standard array geometry with 4.32 "ID $\times 50$ " tall containers of $\mathrm{UF}_{4}$ and Oil with 0.195 " thick Poly walls

\begin{tabular}{|c|c|c|c|c|c|}
\hline \multicolumn{6}{|c|}{ The Effects of Interstitial Water Volume Fraction for a Standard } \\
\hline \multicolumn{6}{|c|}{ Array of $5.25^{\prime \prime}$ ID $\times 15^{\prime \prime}$ Tin/Steel Containers of U308 } \\
\hline Case & $\mathrm{HN}$ & Keff & K_sig & $2^{\star} \mathrm{K}$ sig & Keff+2*K_sig \\
\hline Interstitial & Water $=$ & 0 & & & \\
\hline 71.2 & array-z.05.0 & 0.7402 & 0.0031 & 0.0062 & 0.7464 \\
\hline 33.7 & array-z.10.0 & 0.7533 & 0.0029 & 0.0058 & 0.7591 \\
\hline 21.2 & array-z.15.0 & 0.7612 & 0.0031 & 0.0062 & 0.7674 \\
\hline 15 & array-z.20.0 & 0.7549 & 0.003 & 0.006 & 0.7609 \\
\hline Interstitial & Water $=$ & 0.001 & & & \\
\hline 71.2 & arrz105.out & 0.6981 & 0.0031 & 0.0062 & 0.7043 \\
\hline 33.7 & arrz110.out & 0.725 & 0.0029 & 0.0058 & 0.7308 \\
\hline 21.2 & arrz115.out & 0.7326 & 0.003 & 0.006 & 0.7386 \\
\hline 15 & arrz120.out & 0.7282 & 0.0026 & 0.0052 & 0.7334 \\
\hline Interstitial & Water $=$ & 0.005 & & & \\
\hline 71.2 & arrz205.out & 0.7007 & 0.0027 & 0.0054 & 0.7061 \\
\hline 33.7 & arrz210.out & 0.7281 & 0.0032 & 0.0064 & 0.7345 \\
\hline 21.2 & arrz215.out & 0.7317 & 0.0027 & 0.0054 & 0.7371 \\
\hline 15 & arrz220.out & 0.7282 & 0.0031 & 0.0062 & 0.7344 \\
\hline Interstitial & Water $=$ & 0.01 & & & \\
\hline 71.2 & arrz305.out & 0.694 & 0.003 & 0.006 & 0.7 \\
\hline 33.7 & arrz310.out & 0.7254 & 0.0026 & 0.0052 & 0.7306 \\
\hline 21.2 & arrz315.out & 0.7326 & 0.0029 & 0.0058 & 0.7384 \\
\hline 15 & arrz320.out & 0.7348 & 0.0031 & 0.0062 & 0.741 \\
\hline Interstitial & Water $=$ & 0.05 & & & \\
\hline 71.2 & arrz405.out & 0.7014 & 0.003 & 0.006 & 0.7074 \\
\hline 33.7 & arrz410.out & 0.7255 & 0.0027 & 0.0054 & 0.7309 \\
\hline 21.2 & arrz415.out & 0.7271 & 0.0033 & 0.0066 & 0.7337 \\
\hline 15 & arrz420.out & 0.7285 & 0.0027 & 0.0054 & 0.7339 \\
\hline Interstitial & Water $=$ & 0.1 & - & & \\
\hline 71.2 & arrz505.out & 0.7178 & 0.0026 & 0.0052 & 0.723 \\
\hline 33.7 & arrz510.out & 0.7449 & 0.003 & 0.006 & 0.7509 \\
\hline 21.2 & arrz515.out & 0.7385 & 0.0034 & 0.0068 & 0.7453 \\
\hline 15 & arrz520.out & 0.7334 & 0.0028 & 0.0056 & 0.739 \\
\hline
\end{tabular}


Figure 4.3.1-13. An Array of 4.32" ID $\times 50^{\prime \prime}$ Tall Cylinders of $\mathrm{UO}_{2} \mathrm{~F}_{3}$

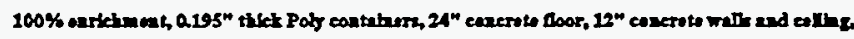

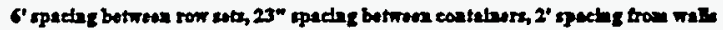

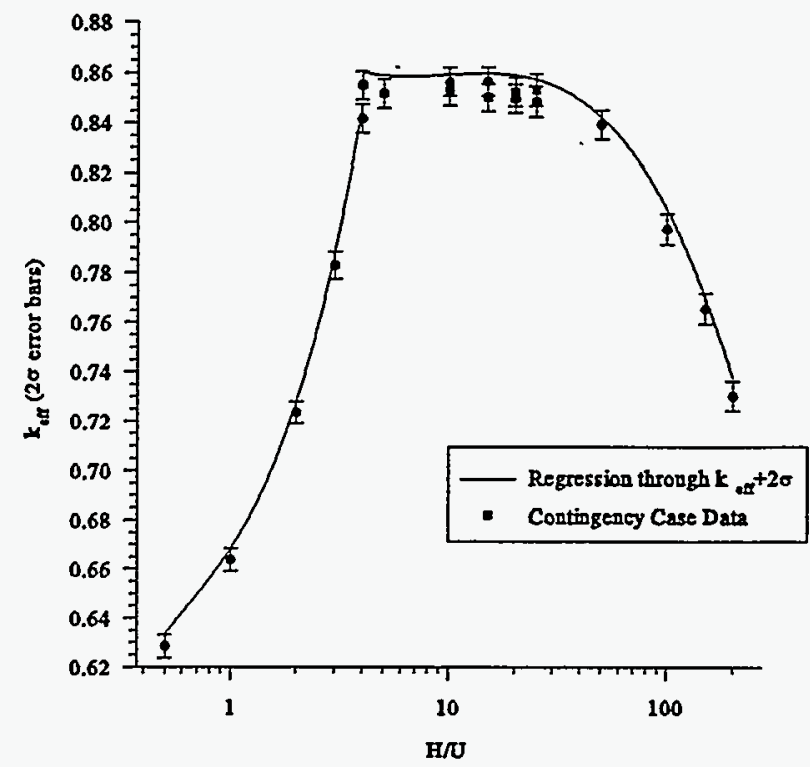

\begin{tabular}{|c|c|c|c|c|c|}
\hline \multicolumn{6}{|c|}{ An Array of 4.32-in. Containers of UO2F2 (Contingency Data) For $100 \%$ Enrichment } \\
\hline Case & $\mathrm{H} / \mathrm{U}$ & Keff & $\bar{K}$ sig & $2^{*} \mathrm{~K}$ sig & Keff $+2^{*} K_{\text {sig }}$ \\
\hline arra01n-c.o & 0.5 & 0.6285 & 0.0024 & 0.0048 & 0.6333 \\
\hline arra02n-c.o & 1 & 0.6637 & 0.0023 & 0.0046 & 0.6683 \\
\hline arra03n-c.o & 2 & 0.7233 & 0.0023 & 0.0046 & 0.7279 \\
\hline arra04n-c.o & 3 & 0.7827 & 0.0027 & 0.0054 & 0.7881 \\
\hline arra05n-c.o & 3.99 & 0.8413 & 0.0029 & 0.0058 & 0.8471 \\
\hline arra06n-c.o & 4 & 0.8546 & 0.0028 & 0.0056 & 0.8602 \\
\hline arra07n-c.o & 5 & 0.8512 & 0.0029 & 0.0058 & 0.857 \\
\hline arra08n-c.o & 10 & 0.8558 & 0.0028 & 0.0056 & 0.8614 \\
\hline arra09n-c.o & 15 & 0.8559 & 0.0029 & 0.0058 & 0.8617 \\
\hline arra10n-c.o & 20 & 0.849 & 0.0028 & 0.0056 & 0.8546 \\
\hline arra11n-c.o & 25 & 0.8479 & 0.0031 & 0.0062 & 0.8541 \\
\hline arra12n-c.o & 50 & 0.8388 & 0.0029 & 0.0058 & 0.8446 \\
\hline arra13n-c.o & 100 & 0.797 & 0.0031 & 0.0062 & 0.8032 \\
\hline arra14n-c.o & 150 & 0.7653 & 0.003 & 0.006 & 0.7713 \\
\hline arra15n-c.o & 200 & 0.7299 & 0.003 & 0.006 & 0.7359 \\
\hline Case & H/U & Keff & K sig & $22^{*}$ K_sig & Keff $+2^{*} \mathrm{~K}$ sig \\
\hline aracno8-c.o & 10 & 0.8526 & 0.0031 & 0.0062 & 0.8588 \\
\hline aracno9-c.o & 15 & 0.8496 & 0.0028 & 0.0056 & 0.8552 \\
\hline $\operatorname{aracn} 10-c .0$ & 20 & 0.8519 & 0.0028 & 0.0056 & 0.8575 \\
\hline $\operatorname{aracn} 11-c .0$ & 25 & 0.8525 & 0.0031 & 0.0062 & 0.8587 \\
\hline
\end{tabular}


Figure 4.3.1-14. An Array of 4.32" I $\times$ 50" Tall Cylinders of UF ^+ Oil

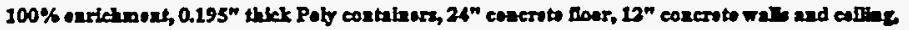

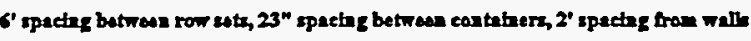

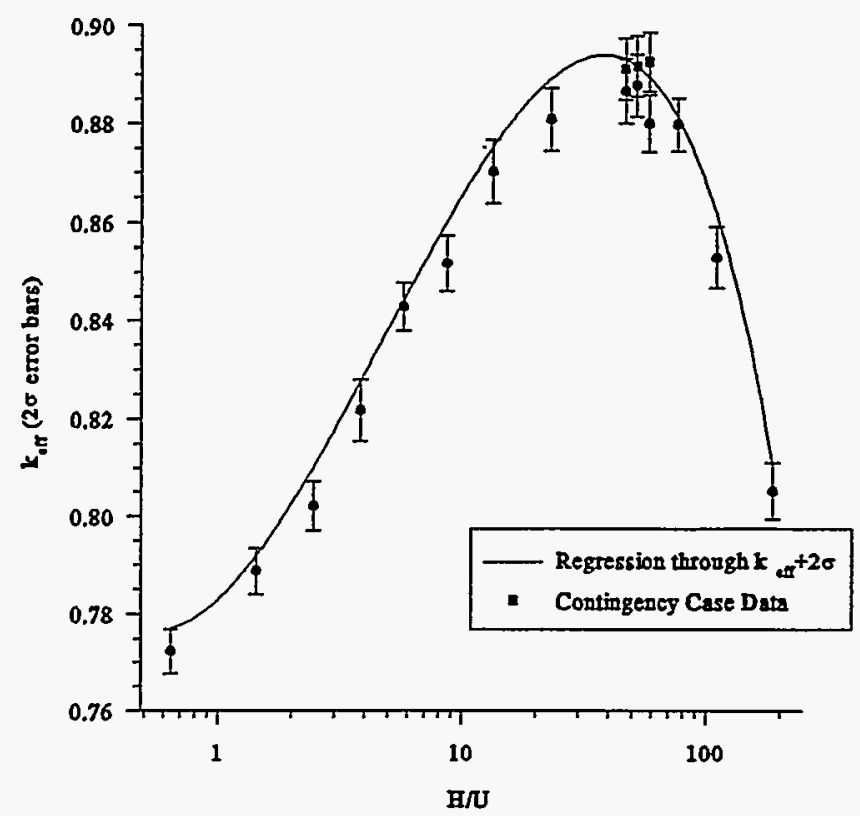

An Array of 4.32-in. Poly. Containers of UF4+Oil For 100\% Enrichment - Overloaded Storage Position

\begin{tabular}{|c|c|c|c|c|c|}
\hline Case & H/U & Keff & K sig & $2^{*} \mathrm{~K}$ sig & Keff+2* K sig \\
\hline arrd01n-c.o & 0.64 & 0.7723 & 0.0023 & 0.0046 & 0.7769 \\
\hline arrd02n-c.o & 1.44 & 0.7887 & 0.0024 & 0.0048 & 0.7935 \\
\hline arrd03n-c.o & 2.47 & 0.8021 & 0.0025 & 0.005 & 0.8071 \\
\hline arrd04n-c.o & 3.84 & 0.8217 & 0.0031 & 0.0062 & 0.8279 \\
\hline arrd05n-c.o & 5.76 & 0.8428 & 0.0025 & 0.005 & 0.8478 \\
\hline arrd06n-c.o & 8.64 & 0.8516 & 0.0028 & 0.0056 & 0.8572 \\
\hline arrd07n-c.o & 13.4 & 0.8701 & 0.0032 & 0.0064 & 0.8765 \\
\hline arrd08n-c.o & 23.1 & 0.8807 & 0.0032 & 0.0064 & 0.8871 \\
\hline arrd09n-c.o & 46.6 & 0.8864 & 0.0033 & 0.0066 & 0.893 \\
\hline ard10n-c.o & 51.9 & 0.8875 & 0.0032 & 0.0064 & 0.8939 \\
\hline arrd11n-c.o & 58.2 & 0.8798 & 0.0029 & 0.0058 & 0.8856 \\
\hline arrd12n-c.o & 76.6 & 0.8796 & 0.0027 & 0.0054 & 0.885 \\
\hline arrd13n-c.o & 109.5 & 0.8529 & 0.0031 & 0.0062 & 0.8591 \\
\hline arrd14n-c.o & 186.4 & 0.8051 & 0.0029 & 0.0058 & 0.8109 \\
\hline Case & H/U & Keff & K sig & $2^{*} \mathrm{~K}$ sig & Keff+2*K sig \\
\hline ardcn09-c.o & 46.6 & 0.8909 & 0.0031 & 0.0062 & 0.8971 \\
\hline arden10-c.o & 51.9 & 0.8915 & 0.0031 & 0.0062 & 0.8977 \\
\hline arden11-c.o & 58.2 & 0.8924 & 0.003 & 0.006 & 0.8984 \\
\hline
\end{tabular}


POEF-SH-30

\subsection{2 $10 \%$ Enriched $\mathrm{UO}_{2} \mathrm{~F}_{2}+\mathrm{H}_{2} \mathrm{O}$ and $\mathrm{UF}_{4}+$ oil}

Table 4.3.2-0 gives the figures and table numbers that contain the result data for this container with various geometry models and fuel materials. The geometry model designators are explained in Table 4.1.1.

The three standard array cases were not analyzed because the $100 \%$ enriched standard array cases fell within the safety criterion. Container thickness was not included in the $10 \%$ enriched cases because all of the more reactive $100 \%$ cases met the safety criterion.

Table 4.3.2-0. Summary of 4.32-inch Container Results for $10 \%$ Enrichment

\begin{tabular}{||c|c|c||}
\hline Geometry Model & Material- & Figure/Table Number \\
\hline \hline SFR & $\mathrm{UO}_{2} \mathrm{~F}_{2}+\mathrm{H}_{2} \mathrm{O}$ & $4.3 .2-1$ \\
\hline $\mathrm{SCC}$ & $\mathrm{UO}_{2} \mathrm{~F}_{2}+\mathrm{H}_{2} \mathrm{O}$ & $4.3 .2-2$ \\
\hline TACW & $\mathrm{UO}_{2} \mathrm{~F}_{2}+\mathrm{H}_{2} \mathrm{O}$ & $4.3 .2-3$ \\
\hline $\mathrm{TSCW}$ & $\mathrm{UO}_{2} \mathrm{~F}_{2}+\mathrm{H}_{2} \mathrm{O}$ & $4.3 .2-4$ \\
\hline $\mathrm{SFR}$ & $\mathrm{UF}_{4}+\mathrm{oil}$ & $4.3 .2-5$ \\
\hline $\mathrm{SCC}$ & $\mathrm{UF}_{4}+\mathrm{oil}$ & $4.3 .2-6$ \\
\hline TACW & $\mathrm{UF}_{4}+\mathrm{oil}$ & $4.3 .2-7$ \\
\hline TSCW & $\mathrm{UF}_{4}+\mathrm{oil}$ & $4.3 .2-8$ \\
\hline
\end{tabular}


Figure 4.3.2-1. Single Fully Reflected 4.32" ID $~$ 50" Tall Cylinder of UO ${ }_{2} \mathrm{~F}_{2}$ $10 \%$ enrlebment, 24" conerete foer, 12" (minlanm) water rethector on all othur wdes

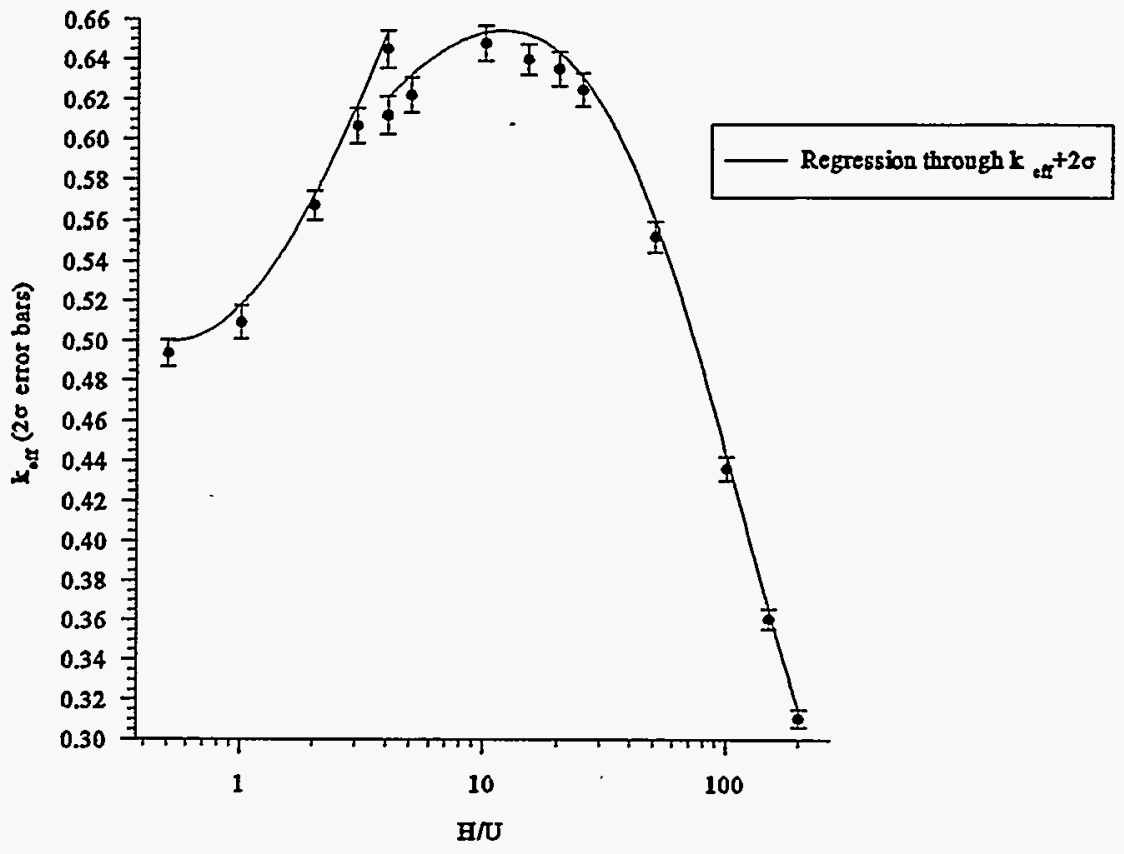

Single 4.32 in. Diameter Poly. Container w/ Full Reflection for $10 \%$ Enriched $\mathrm{UO} 2 \mathrm{~F} 2+\mathrm{H} 2 \mathrm{O}$

\begin{tabular}{|c|c|c|c|c|c|}
\hline \hline Case & H/U & Keff & K_sig & $2^{\star} \mathrm{K}$ sig & Keff+2*K sig \\
\hline \hline fru001n.o & 0.5 & 0.4938 & 0.0034 & 0.0068 & 0.5006 \\
\hline fru002n.o & 1 & 0.5092 & 0.0041 & 0.0082 & 0.5174 \\
\hline fru003n.o & 2 & 0.5672 & 0.0036 & 0.0072 & 0.5744 \\
\hline fru004n.o & 3 & 0.6064 & 0.0044 & 0.0088 & 0.6152 \\
\hline fru005n.o & 3.99 & 0.6446 & 0.0046 & 0.0092 & 0.6538 \\
\hline fru006n.o & 4 & 0.6117 & 0.0048 & 0.0096 & 0.6213 \\
\hline fru007n.o & 5 & 0.6219 & 0.0043 & 0.0086 & 0.6305 \\
\hline fru008n.o & 10 & 0.6475 & 0.0044 & 0.0088 & 0.6563 \\
\hline fru009n.o & 15 & 0.6395 & 0.0038 & 0.0076 & 0.6471 \\
\hline fru010n.o & 20 & 0.6348 & 0.0043 & 0.0086 & 0.6434 \\
\hline fru011n.o & 25 & 0.6243 & 0.0041 & 0.0082 & 0.6325 \\
\hline fru012n.o & 50 & 0.5517 & 0.0037 & 0.0074 & 0.5591 \\
\hline fru013n.o & 100 & 0.4362 & 0.0029 & 0.0058 & 0.442 \\
\hline fru014n.o & 150 & 0.3605 & 0.0026 & 0.0052 & 0.3657 \\
\hline fru015n.o & 200 & 0.3106 & 0.0022 & 0.0044 & 0.315 \\
\hline
\end{tabular}


Figure 4.3.2-2. Single 4.32" ID $\times 50$ " Tall Cylinder of $\mathrm{UO}_{2} \mathrm{~F}_{2}$ in a Concrete Corner $10 \%$ enrichment, 24 " concrete floor, 12 " concrete walls and ceiling, $-100 \mathrm{~kg}$ water reflector simulating a person

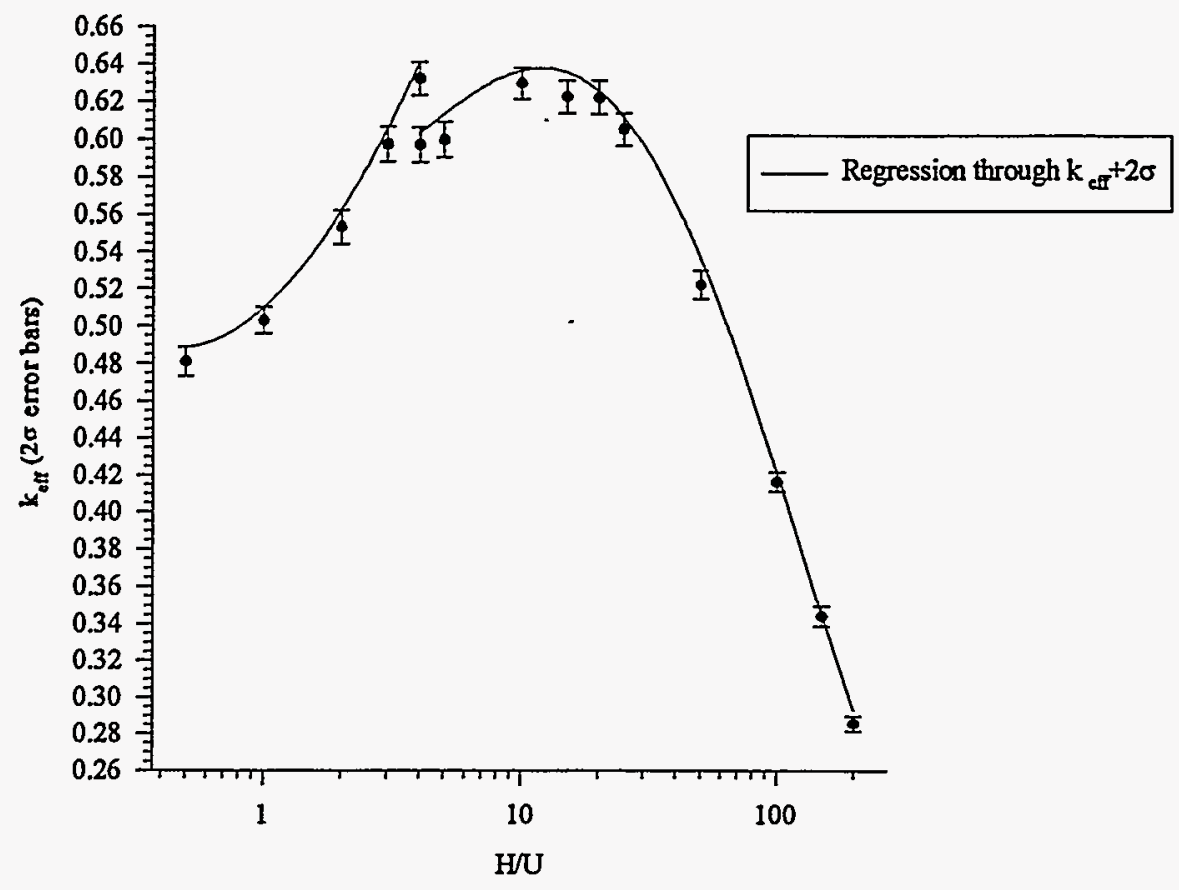

Single 4.32 in. Diameter Poly. Container in a Corner for 10\% Enriched UO2F2+H2O

\begin{tabular}{|c|c|c|c|c|c|}
\hline \hline Case & H/N & Keff & K_sig & $2^{\star}$ K_sig & Keff+2*K_sig \\
\hline cpu001n.o & 0.5 & 0.4811 & 0.0038 & 0.0076 & 0.4887 \\
\hline cpu002n.o & 1 & 0.5031 & 0.0035 & 0.007 & 0.5101 \\
\hline cpu003n.o & 2 & 0.5533 & 0.0044 & 0.0088 & 0.5621 \\
\hline cpu004n.o & 3 & 0.5969 & 0.0047 & 0.0094 & 0.6063 \\
\hline cpu005n.o & 3.99 & 0.6316 & 0.0044 & 0.0088 & 0.6404 \\
\hline cpu006n.o & 4 & 0.5965 & 0.0047 & 0.0094 & 0.6059 \\
\hline cpu007n.o & 5 & 0.5992 & 0.0046 & 0.0092 & 0.6084 \\
\hline cpu008n.o & 10 & 0.6289 & 0.0042 & 0.0084 & 0.6373 \\
\hline cpu009n.o & 15 & 0.6217 & 0.0043 & 0.0086 & 0.6303 \\
\hline cpu010n.o & 20 & 0.6214 & 0.0044 & 0.0088 & 0.6302 \\
\hline cpu011n.o & 25 & 0.6046 & 0.0043 & 0.0086 & 0.6132 \\
\hline cpu012n.o & 50 & 0.5216 & 0.0038 & 0.0076 & 0.5292 \\
\hline cpu013n.o & 100 & 0.4162 & 0.0027 & 0.0054 & 0.4216 \\
\hline cpu014n.o & 150 & 0.3441 & 0.0027 & 0.0054 & 0.3495 \\
\hline cpu015n.o & 200 & 0.2854 & 0.0021 & 0.0042 & 0.2896 \\
\hline
\end{tabular}


Figure 4.3.2-3. Two 4.32" $\mathrm{W} \times 50^{\prime \prime}$ Tall Cylinders of $\mathrm{UO}_{2} \mathrm{~F}_{2} \mathrm{Next}$ to a $\mathrm{W}$ all $10 \%$ enrichment, $24^{\prime \prime}$ conerete floor, 12 " concrete wall and celling, $106 \mathrm{~kg}$ water reflector atmulating a person

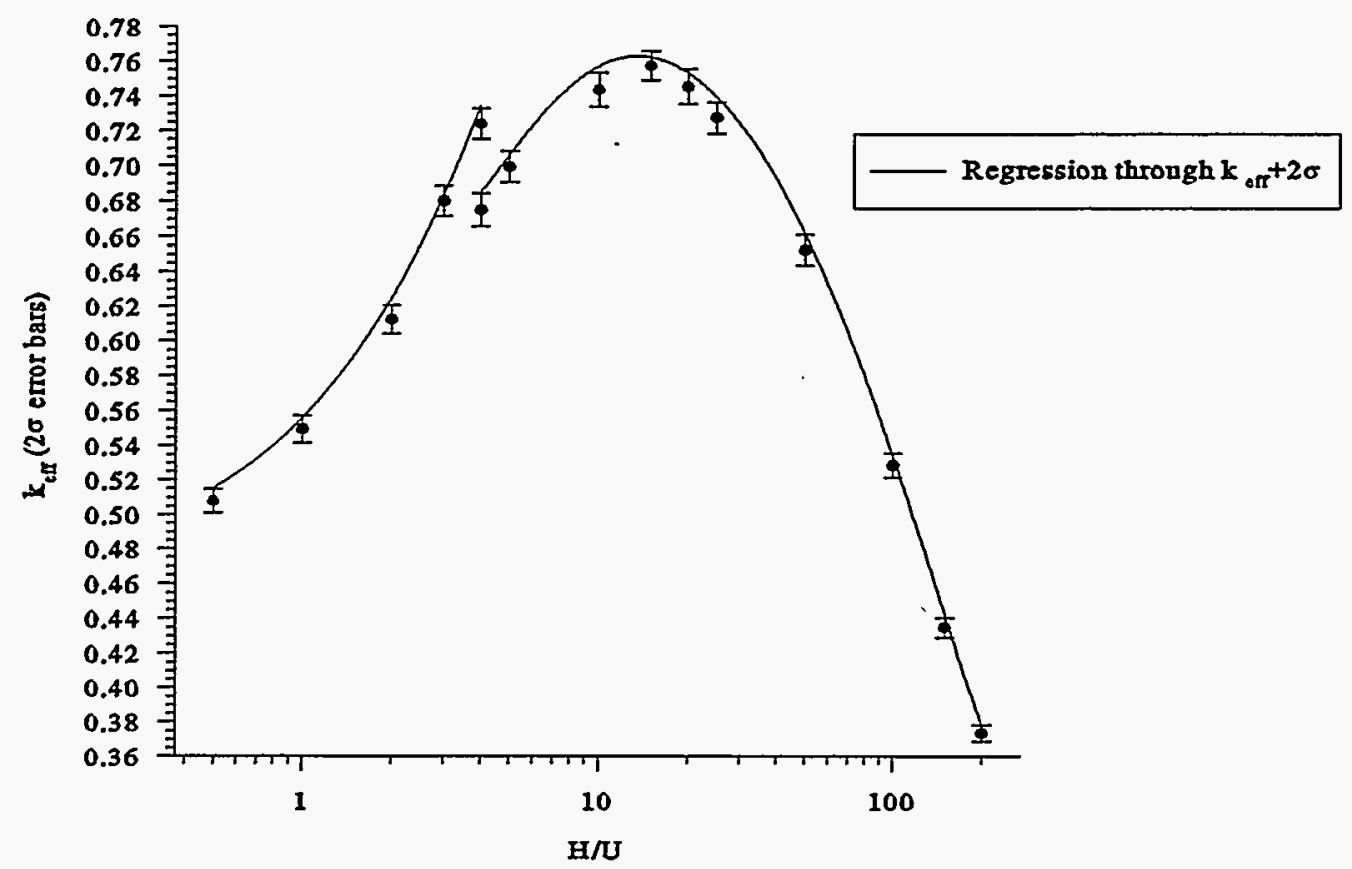

Two 4.32 in. Diameter Poly. Containers Adjacent to Concrete Wall for $10 \%$ Enriched UO2F2+H2O

\begin{tabular}{|c|c|c|c|c|c|}
\hline \hline Case & H/U & Keff & K_sig & $2^{\star}$ K sig & Keff+2*K sig \\
\hline npu001n.o & 0.5 & 0.5079 & 0.0035 & 0.007 & 0.5149 \\
\hline npu002n.o & 1 & 0.5496 & 0.0039 & 0.0078 & 0.5574 \\
\hline npu003n.o & 2 & 0.6123 & 0.0041 & 0.0082 & 0.6205 \\
\hline npu004n.o & 3 & 0.6799 & 0.0043 & 0.0086 & 0.6885 \\
\hline npu005n.o & 3.99 & 0.7235 & 0.0043 & 0.0086 & 0.7321 \\
\hline npu006n.o & 4 & 0.6747 & 0.0047 & 0.0094 & 0.6841 \\
\hline npu007n.o & 5 & 0.6991 & 0.0043 & 0.0086 & 0.7077 \\
\hline npu008n.o & 10 & 0.7429 & 0.0049 & 0.0098 & 0.7527 \\
\hline npu009n.o & 15 & 0.7566 & 0.0042 & 0.0084 & 0.765 \\
\hline npu010n.o & 20 & 0.7447 & 0.0049 & 0.0098 & 0.7545 \\
\hline npu011n.o & 25 & 0.7269 & 0.0045 & 0.009 & 0.7359 \\
\hline npu012n.o & 50 & 0.6518 & 0.0043 & 0.0086 & 0.6604 \\
\hline npu013n.o & 100 & 0.5281 & 0.0035 & 0.007 & 0.5351 \\
\hline npu014n.o & 150 & 0.4346 & 0.0027 & 0.0054 & 0.44 \\
\hline npu015n.o & 200 & 0.3736 & 0.0024 & 0.0048 & 0.3784 \\
\hline
\end{tabular}


Figure 4.3.2-4. Two 4.32" ID $\times 50 "$ Tall Cylinders of $\mathrm{UO}_{2} \mathrm{~F}_{2}$ One Foot from a $\mathrm{Wall}$

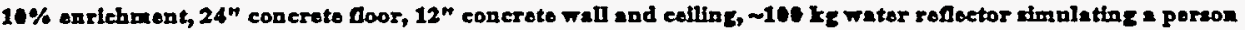

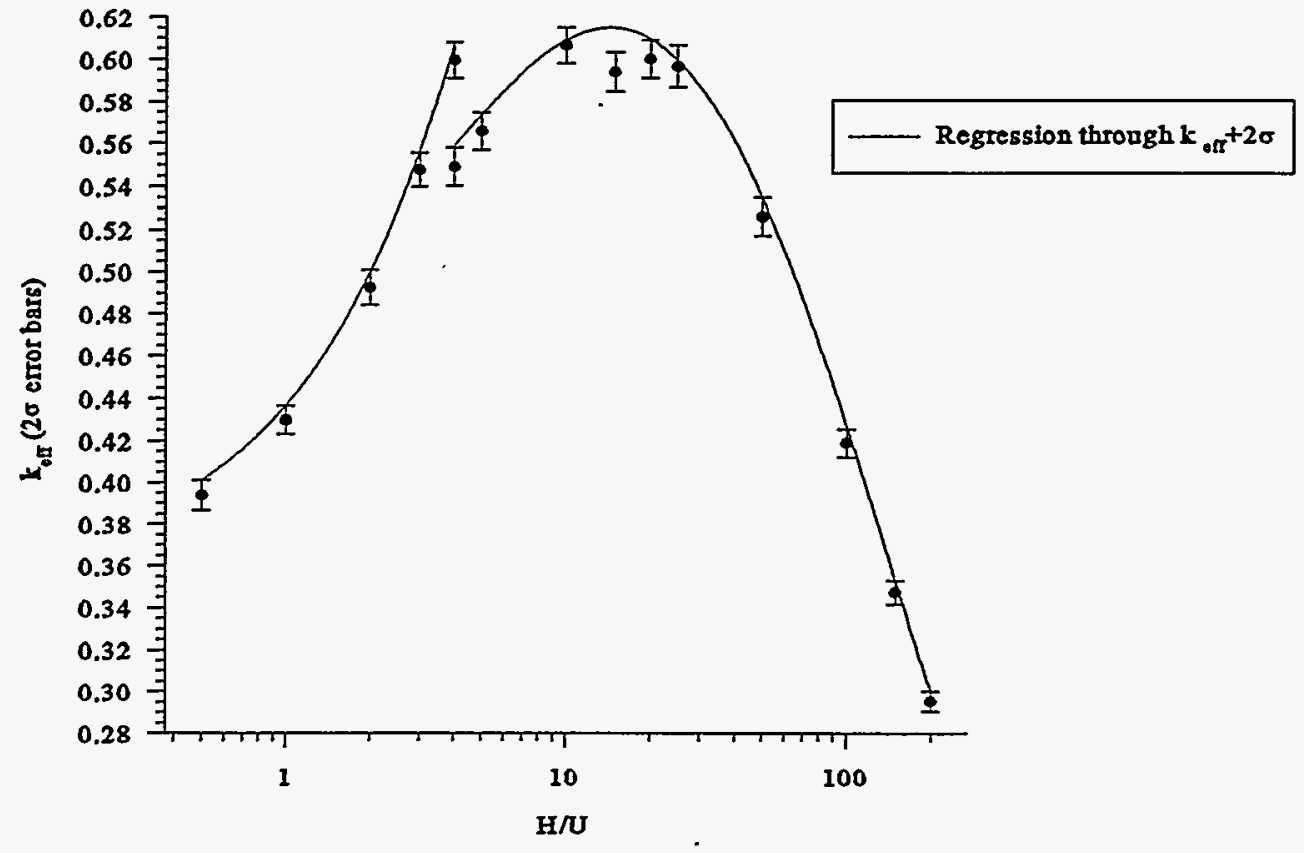

\begin{tabular}{|c|c|c|c|c|c|}
\hline \multicolumn{6}{|c|}{ for $10 \%$ Enriched UO2F $2+\mathrm{H} 2 \mathrm{O}$} \\
\hline Case & $\mathrm{H} / \mathrm{U}$ & Keff & K_sig & 2*K_sig & Keff $+2 \star K$ sig \\
\hline apu001n.o & 0.5 & 0.3938 & 0.0036 & 0.0072 & 0.401 \\
\hline apu002n.o & 1 & 0.4297 & 0.0033 & 0.0066 & 0.4363 \\
\hline apu003n.o & 2 & 0.4925 & 0.0041 & 0.0082 & 0.5007 \\
\hline apu004n.o & 3 & 0.5475 & 0.0039 & 0.0078 & 0.5553 \\
\hline apu005n.o & 3.99 & 0.5991 & 0.0042 & 0.0084 & 0.6075 \\
\hline apu006n.o & 4 & 0.5489 & 0.0044 & 0.0088 & 0.5577 \\
\hline apu007n.o & 5 & 0.5654 & 0.0044 & 0.0088 & 0.5742 \\
\hline apu008n.o & 10 & 0.6061 & 0.0042 & 0.0084 & 0.6145 \\
\hline apu009n.o & 15 & 0.5934 & 0.0046 & 0.0092 & 0.6026 \\
\hline apu010n.o & 20 & 0.5996 & 0.0044 & 0.0088 & 0.6084 \\
\hline apu011n.o & 25 & 0.596 & 0.0049 & 0.0098 & 0.6058 \\
\hline apu012n.o & 50 & 0.5257 & 0.0044 & 0.0088 & 0.5345 \\
\hline apu013n.o & 100 & 0.4186 & 0.0033 & 0.0066 & 0.4252 \\
\hline apu014n.o & 150 & 0.3473 & 0.0028 & 0.0056 & 0.3529 \\
\hline apu015n.o & 200 & 0.2953 & 0.0024 & 0.0048 & 0.3001 \\
\hline
\end{tabular}


POEF-SH-30

Figure 4.3.2-5. Single Fully Reflected 4.32" ID × 50" Tall Cylinder of UF , and Oil

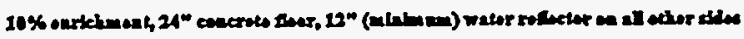

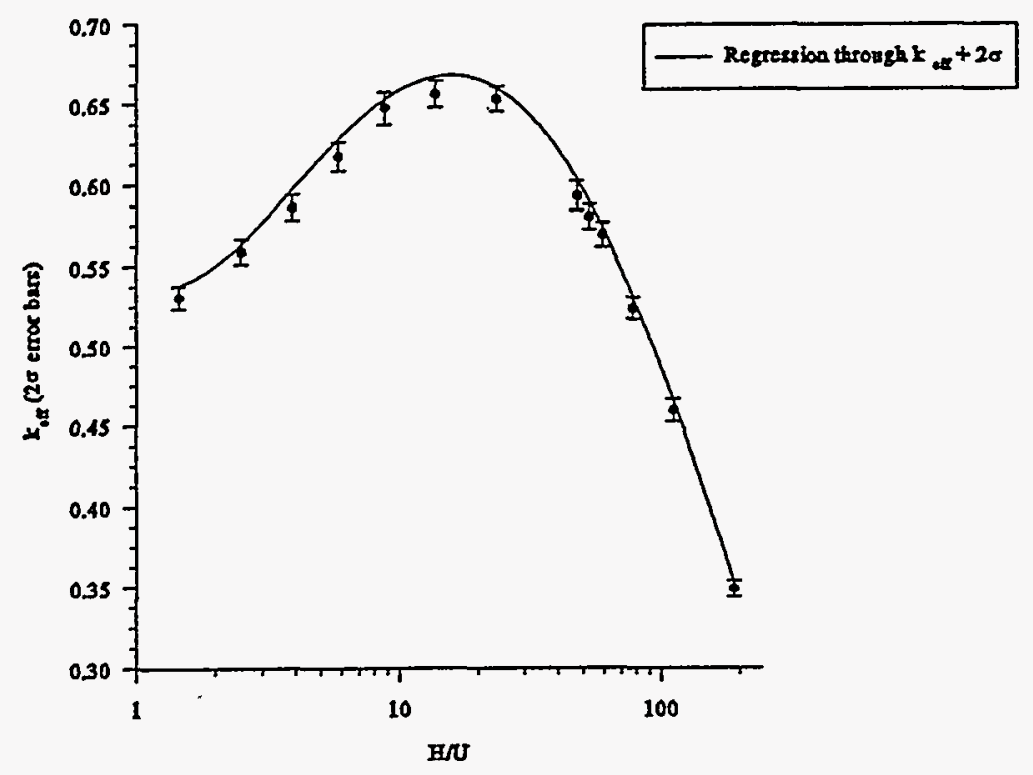

\begin{tabular}{|c|c|c|c|r|c|}
\hline \multicolumn{6}{|l|}{ Single 4.32 in Diameter poly container w/ Full Reflection } \\
\hline for 10\% enriched UF4+oil & & & & \\
\hline & & & & & \\
\hline Case & H/U & Keff & K sig & \multicolumn{1}{|c|}{$2^{\star}$ K sig } & Keff +2*K sig \\
\hline & & & & & \\
\hline sfr-432.p65.0 & 0.65 & 0.50459 & 0.00362 & 0.00724 & 0.51183 \\
\hline sfr-432.145.0 & 1.45 & 0.5308 & 0.0035 & 0.007 & 0.5378 \\
\hline sfr-432.249.0 & 2.49 & 0.55949 & 0.00394 & 0.00788 & 0.56737 \\
\hline sfr-432.388.0 & 3.88 & 0.58617 & 0.00393 & 0.00786 & 0.59403 \\
\hline sfr-432.582.0 & 5.82 & 0.61712 & 0.00452 & 0.00904 & 0.62616 \\
\hline sfr-432.873.0 & 8.73 & 0.64738 & 0.00522 & 0.01044 & 0.65782 \\
\hline sfr-432.136:0 & 13.6 & 0.65605 & 0.00427 & 0.00854 & 0.66459 \\
\hline sfr-432.233.0 & 23.3 & 0.65272 & 0.00394 & 0.00788 & 0.6606 \\
\hline sfr-432.471.0 & 47.1 & 0.59258 & 0.00444 & 0.00888 & 0.60146 \\
\hline sfr-432.524.0 & 52.4 & 0.57976 & 0.00377 & 0.00754 & 0.5873 \\
\hline sfr-432.588.0 & 58.8 & 0.56906 & 0.00363 & 0.00726 & 0.57632 \\
\hline sfr-432.773.0 & 77.3 & 0.52313 & 0.00344 & 0.00688 & 0.53001 \\
\hline sfr-432.110.0 & 110.6 & 0.45904 & 0.00345 & 0.0069 & 0.46594 \\
\hline sfr-432.188.0 & 188.2 & 0.34878 & 0.0025 & 0.005 & 0.35378 \\
\hline
\end{tabular}


POEF-SH-30

Figure 4.3.2-6. Single 4.32" ID $\times$ 50" Tall Cylinder of UF ${ }_{4}$ and Oil in a Concrete Corner

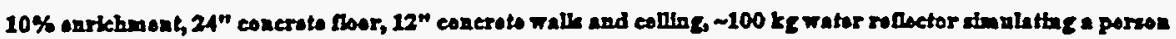

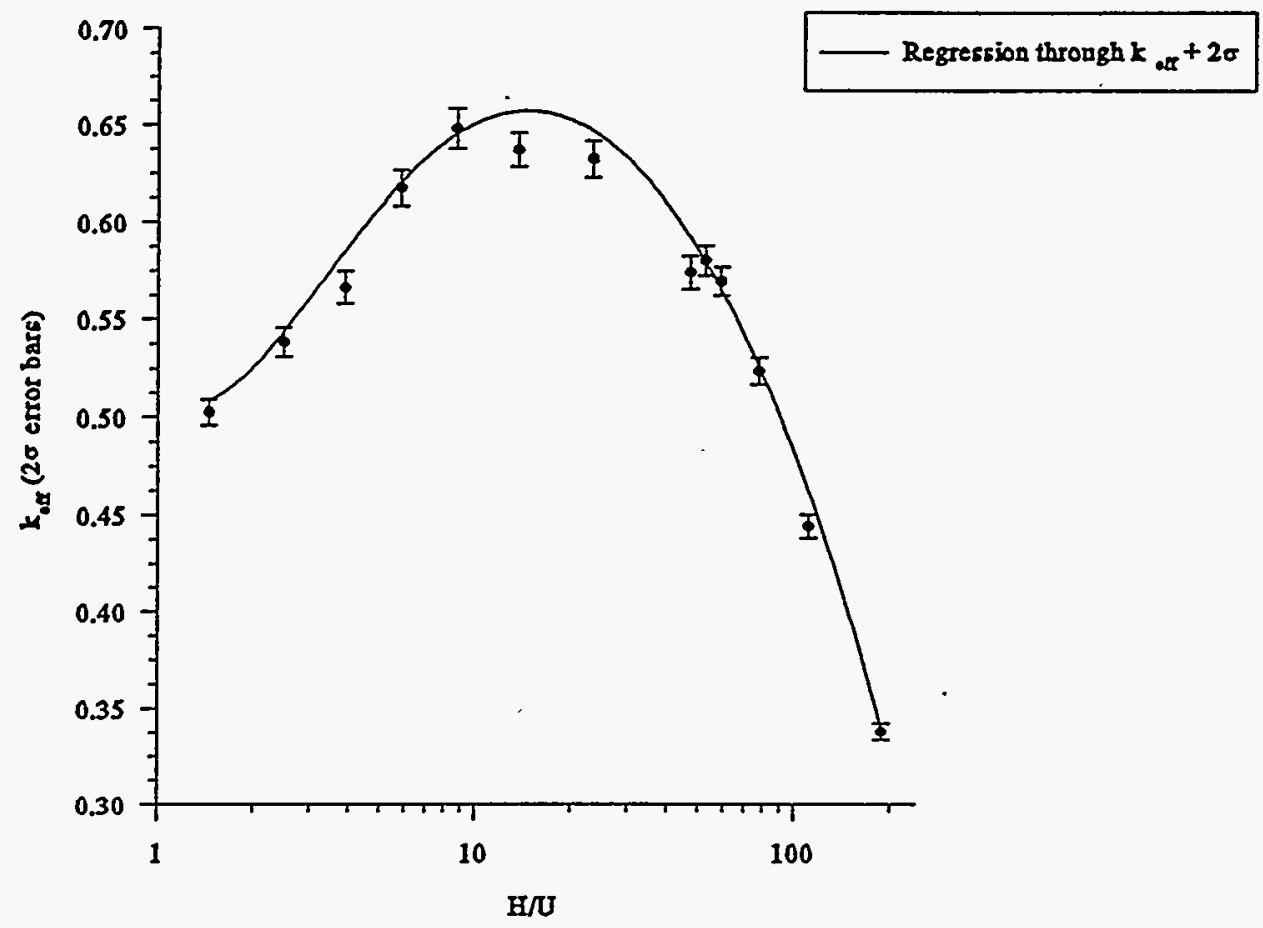

\begin{tabular}{|c|c|c|c|c|c|}
\hline \multicolumn{3}{|c|}{ For $10 \%$ Enriched UF4+oil } & & & \\
\hline Case & HIS & Keff & K sig & $2^{\star} \mathrm{K}$ sig & Keff+2sig \\
\hline $\operatorname{scc}-432.145 .0$ & 1.45 & 0.50244 & 0.00324 & 0.00648 & 0.50892 \\
\hline scc-432.249.o & 2.49 & 0.5382 & 0.00371 & 0.00742 & 0.54562 \\
\hline scc- 432.388 .0 & 3.88 & 0.5662 & 0.00426 & 0.00852 & 0.57472 \\
\hline scc-432.582.0 & 5.82 & 0.61712 & 0.00452 & 0.00904 & 0.62616 \\
\hline scc-432.873.0 & 8.73 & 0.64738 & 0.00522 & 0.01044 & 0.65782 \\
\hline scc-432.136.o & 13.6 & 0.63631 & 0.00445 & 0.0089 & 0.64521 \\
\hline scc-432.233.o & 23.3 & 0.63145 & 0.00471 & 0.00942 & 0.64087 \\
\hline scc-432.471.o & 47.1 & 0.57369 & 0.00421 & 0.00842 & 0.58211 \\
\hline scc-432.524.o & 52.4 & 0.57976 & 0.00377 & 0.00754 & 0.5873 \\
\hline scc- 432.588 .0 & 58.8 & 0.56906 & 0.00363 & 0.00726 & 0.57632 \\
\hline $\operatorname{scc}-432.773 .0$ & 77.3 & 0.52313 & 0.00344 & 0.00688 & 0.53001 \\
\hline $\operatorname{scc}-432.110 .0$ & 110.6 & 0.4439 & 0.00302 & 0.00604 & 0.44994 \\
\hline $\operatorname{scc}-432.188 .0$ & 188.2 & 0.33789 & 0.00209 & 0.00418 & 0.34207 \\
\hline
\end{tabular}


Figure 4.3.2-7. Two 4.32" ID $\times 50$ "Tall Cylinders of $\mathrm{UF}_{4}$ and Oil Next to a Wall $10 \%$ enrichment, $24^{\prime \prime}$ concrete floor, 12 " concrete wall and ceiling, $\sim 100 \mathrm{~kg}$ water reflector simulating a person

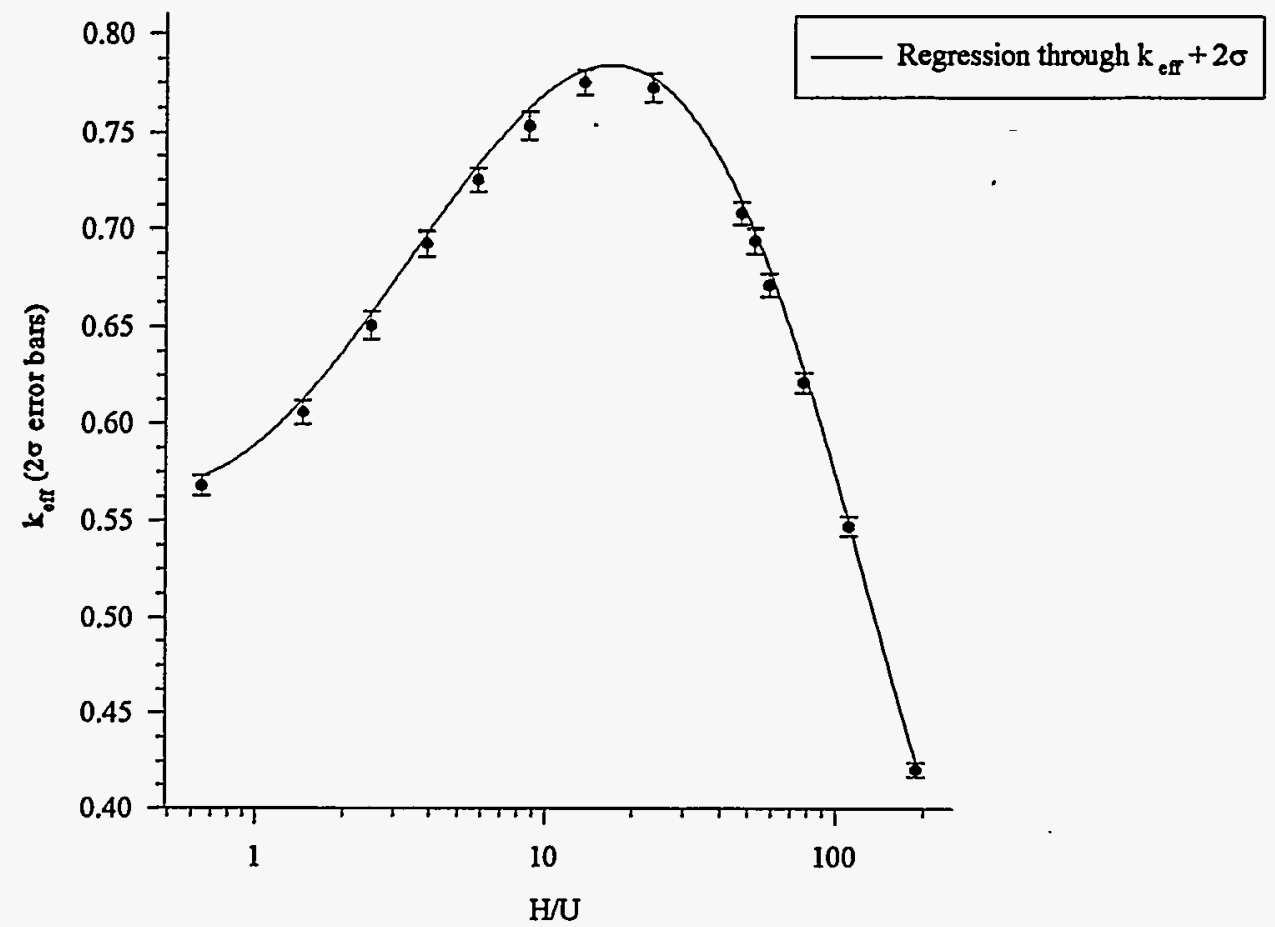

\begin{tabular}{|c|c|c|c|c|c|}
\hline \multicolumn{5}{|c|}{ Two 4.32 in. Diameter Poly. Containers Adjacent to Concrete Wall } & for $10 \%$ Enriched UF4+oil \\
\hline Case & $\overline{\mathrm{H} / \mathrm{U}}$ & Keff & K_sig & 2*K_sig & Keff $+2^{*}$ K sig \\
\hline do0n-432.p65 & 0.65 & 0.5679 & 0.00252 & 0.00504 & 0.57294 \\
\hline$d 00 n-432.145$ & 1.45 & 0.6059 & 0.003 & 0.006 & 0.6119 \\
\hline d00n-432.249 & 2.49 & 0.6506 & 0.00349 & 0.00698 & 0.65758 \\
\hline do0n-432.388 & 3.88 & 0.6924 & 0.00329 & 0.00658 & 0.69898 \\
\hline doon-432.582 & 5.82 & 0.7251 & 0.00309 & 0.00618 & 0.73128 \\
\hline do0n-432.873 & 8.73 & 0.753 & 0.00355 & 0.0071 & 0.7601 \\
\hline$d 00 n-432.136$ & 13.6 & 0.775 & 0.00318 & 0.00636 & 0.78136 \\
\hline$d 00 n-432.233$ & 23.3 & 0.7724 & 0.00363 & 0.00726 & 0.77966 \\
\hline do0n-432.471 & 47.1 & 0.7079 & 0.00291 & 0.00582 & 0.71372 \\
\hline doon-432.524 & 52.4 & 0.6936 & 0.0032 & 0.0064 & 0.7 \\
\hline$d 00 n-432.588$ & 58.8 & 0.6712 & 0.00293 & 0.00586 & 0.67706 \\
\hline do0n-432.773 & 77.3 & 0.6212 & 0.00255 & 0.0051 & 0.6263 \\
\hline doon-432.110 & 110.6 & 0.5472 & 0.00246 & 0.00492 & 0.55212 \\
\hline $\mathrm{d} 00 \mathrm{n}-432.188$ & 188.2 & 0.4207 & 0.00175 & 0.0035 & 0.4242 \\
\hline
\end{tabular}


Figure 4.3.2-8. Two 4.32" ID x 50" Tall Cylinders of UP , and Oil One Foot from a Wall

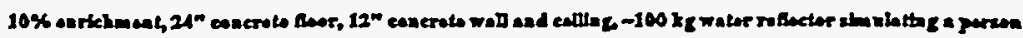

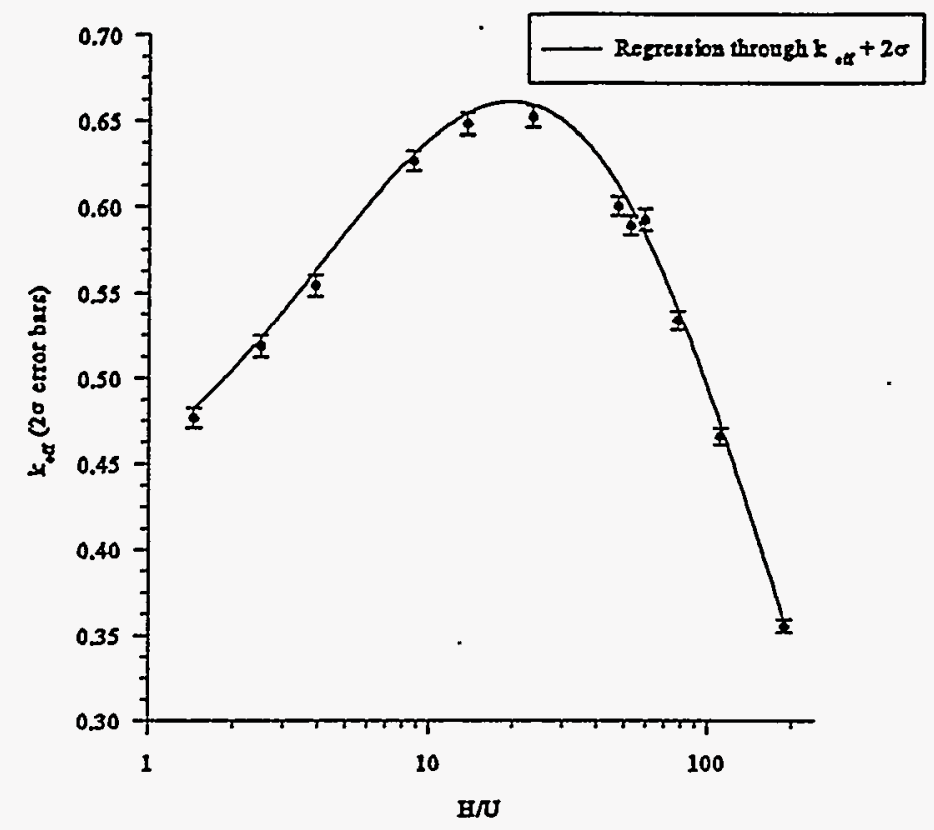

\begin{tabular}{|c|c|c|c|c|c|}
\hline \multicolumn{6}{|c|}{ Two 4.32 in. Diameter Poly. Containers 1 ' from Concrete Wall } \\
\hline \multicolumn{3}{|c|}{ for $10 \%$ Enriched UF4+oil } & & & \\
\hline Case & $\mathrm{H} / \mathrm{U}$ & Keff & K sig & $2 * K$ sig & Keff $+2^{*} \mathrm{~K}$ sig \\
\hline$d 12 n-432 . p 65.0$ & 0.65 & 0.44499 & 0.00257 & 0.00514 & 0.45013 \\
\hline$d 12 n-432.145 .0$ & 1.45 & 0.47623 & 0.00299 & 0.00598 & 0.48221 \\
\hline$d 12 n-432.249 .0$ & 2.49 & 0.51829 & 0.00312 & 0.00624 & 0.52453 \\
\hline $\mathrm{d} 12 \mathrm{n}-432.388 .0$ & 3.88 & 0.55363 & 0.00311 & 0.00622 & 0.55985 \\
\hline$d 12 n-432.873 .0$ & 8.73 & 0.62622 & 0.00295 & 0.0059 & 0.63212 \\
\hline$d 12 n-432.136 .0$ & 13.6 & 0.64784 & 0.00318 & 0.00636 & 0.6542 \\
\hline$d 12 n-432.233 .0$ & 23.3 & 0.65206 & 0.00311 & 0.00622 & 0.65828 \\
\hline d12n-432.471.o & 47.1 & 0.59997 & 0.00271 & 0.00542 & 0.60539 \\
\hline d $12 n-432.524 .0$ & 52.4 & 0.58895 & 0.00282 & 0.00564 & 0.59459 \\
\hline d12n-432.588.0 & 58.8 & 0.59217 & 0.00327 & 0.00654 & 0.59871 \\
\hline d12n-432.773.o & 77.3 & 0.53367 & 0.00271 & 0.00542 & 0.53909 \\
\hline d12n-432.110.0 & 110.6 & 0.46546 & 0.00238 & 0.00476 & 0.47022 \\
\hline$d 12 n-432.188 .0$ & 188.2 & 0.35535 & 0.00178 & 0.00356 & 0.35891 \\
\hline
\end{tabular}




\subsection{0-inch-Diameter by 24-inch-Tall Polyethylene Container}

This section presents results of calculations designed to model the "24-inch polybottle," "GP container," and "CO-4 polybottle" containers described in Table 1.0-1.

\subsection{1 $100 \%$ Enriched $\mathrm{UO}_{2} \mathrm{~F}_{2}+\mathrm{H}_{2} \mathrm{O}$ and $\mathrm{UF}_{4}+\mathrm{Oil}$}

Table 4.4.1-0 gives the figures and table numbers that contain the results data for this container with various geometry models and fuel materials. The geometry model designators are explained in Table 4.1-1. Some of the $\mathrm{UO}_{2} \mathrm{~F}_{2}$ cases were reevaluated using a constant uranium density of $3.2 \mathrm{gU} / \mathrm{cc}$ as discussed in Section 4.1. Where this option was used, the titles of the figures reflect the difference. The TSCW $\mathrm{UO}_{2} \mathrm{~F}_{2}+\mathrm{H}_{2} \mathrm{O}$ case includes the maximum polybottle thickness. The other cases effected by bottle thickness were not evaluated because original (no poly) cases failed the safety criterion. The result of overloading a storage position in an array are shown as data points on the $\mathrm{k}_{\text {eff }}$ versus $\mathrm{H} / \mathrm{U}$ graphs. The plotted curve is through the $k_{\text {eff }}+2 \sigma$ data for the standard array. 
Table 4.4.1-0. Summary of 5.0-inch References for $100 \%$ Enrichment

\begin{tabular}{|c|c|c||}
\hline Geometry Model & Material & Figure/Table Number \\
\hline \hline SFR & $\mathrm{UO}_{2} \mathrm{~F}_{2}+\mathrm{H}_{2} \mathrm{O}$ & $4.4 .1-1$ \\
\hline $\mathrm{SCC}$ & $\mathrm{UO}_{2} \mathrm{~F}_{2}+\mathrm{H}_{2} \mathrm{O}$ & $4.4 .1-2$ \\
\hline TACW & $\mathrm{UO}_{2} \mathrm{~F}_{2}+\mathrm{H}_{2} \mathrm{O}$ & $4.4 .1-3$ \\
\hline TSCW & $\mathrm{UO}_{2} \mathrm{~F}_{2}+\mathrm{H}_{2} \mathrm{O}$ & $4.4 .1-4$ \\
\hline SFR & $\mathrm{UF}_{4}+$ oil & $4.4 .1-5$ \\
\hline SCC & $\mathrm{UF}_{4}+$ oil & $4.4 .1-6$ \\
\hline TACW & $\mathrm{UF}_{4}+$ oil & $4.4 .1-7$ \\
\hline TSCW & $\mathrm{UF}_{4}+$ oil & $4.4 .1-8$ \\
\hline ACE & $\mathrm{UO}_{2} \mathrm{~F}_{2}+\mathrm{H}_{2} \mathrm{O}$ & $4.4 .1-9$ \\
\hline ACE & $\mathrm{UF}_{4}+\mathrm{oil}_{1}$ & $4.4 .1-10$ \\
\hline AI & $\mathrm{UO}_{2} \mathrm{~F}_{2}+\mathrm{H}_{2} \mathrm{O}$ & $4.4 .1-11$ \\
\hline AOSP & $\mathrm{UO}_{2} \mathrm{~F}_{2}+\mathrm{H}_{2} \mathrm{O}$ & $4.4 .1-12$ \\
\hline
\end{tabular}


Figure 4.4.1-1. Single Fully Reflected 5.00" ID $\times 24^{\prime \prime}$ Tall Cylinder of UO ${ }_{2} \mathrm{~F}_{2}$

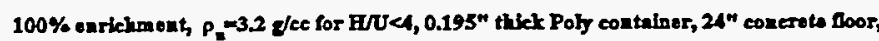
$12 "$ (mingimem) water resoctor on all otker sides

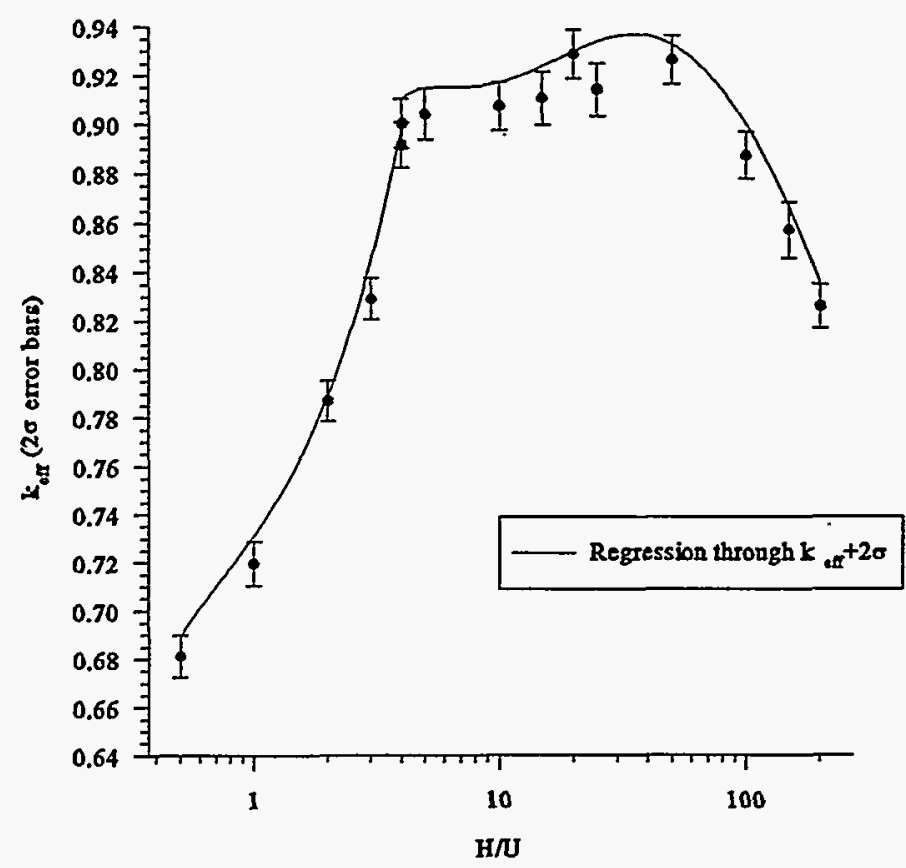

Single 5.0 in. Diameter Poly. Container w/ Full Reflection for UO2F2 $+\mathrm{H} 2 \mathrm{O}$, Uranium Density $=3.2 \mathrm{gU} / \mathrm{cc}$

\begin{tabular}{|c|c|c|c|c|c|}
\hline \hline Case & H/U & Keff & K sig & $2^{*}$ K sig & Keff+2*K_sig \\
\hline frb01n-c & 0.5 & 0.681 & 0.0044 & 0.0088 & 0.6898 \\
\hline frb02n-c & 1 & 0.7196 & 0.0047 & 0.0094 & 0.729 \\
\hline frb03n-c & 2 & 0.7868 & 0.0042 & 0.0084 & 0.7952 \\
\hline frb04n-c & 3 & 0.8287 & 0.0042 & 0.0084 & 0.8371 \\
\hline frb05n-c & 3.99 & 0.8912 & 0.0046 & 0.0092 & 0.9004 \\
\hline \hline frb06n-c & 4 & 0.8999 & 0.005 & 0.01 & 0.9099 \\
\hline frb07n-c & 5 & 0.9037 & 0.0051 & 0.0102 & 0.9139 \\
\hline frb08n-c & 10 & 0.9068 & 0.0049 & 0.0098 & 0.9166 \\
\hline frb09n-c & 15 & 0.9098 & 0.0054 & 0.0108 & 0.9206 \\
\hline frb10n-c & 20 & 0.9279 & 0.005 & 0.01 & 0.9379 \\
\hline frb11n-c & 25 & 0.9134 & 0.0054 & 0.0108 & 0.9242 \\
\hline frb12n-c & 50 & 0.9254 & 0.005 & 0.01 & 0.9354 \\
\hline frb13n-c & 100 & 0.8865 & 0.0048 & 0.0096 & 0.8961 \\
\hline frb14n-c & 150 & 0.8563 & 0.0057 & 0.0114 & 0.8677 \\
\hline frb15n-c & 200 & 0.8252 & 0.0045 & 0.009 & 0.8342 \\
\hline
\end{tabular}


Figure 4.4.1-2. Single 5.00" $\mathrm{ID} \times 24$ " Tall Cylinder of $\mathrm{UO}_{2} \mathrm{~F}_{2}$ in a Concrete Corner

$100 \%$ enrichment, $p_{z}=3.2 \mathrm{~g} / \mathrm{cc}$ for $\mathrm{H} / \mathrm{U}<4,0.195^{\prime \prime}$ thick Poły container, 24 " concrete floor,

12 " concrete walls and ceiling, $\sim 100 \mathrm{~kg}$ water reflector simulating 2 person

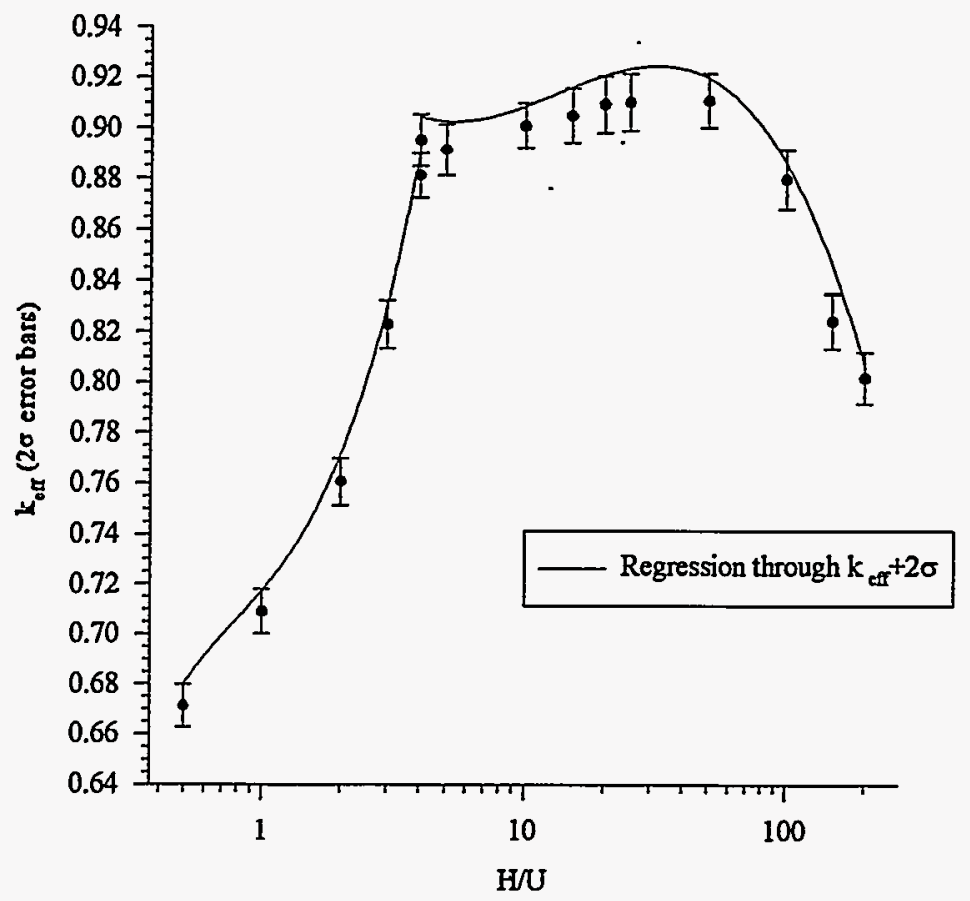

\begin{tabular}{|c|c|c|c|c|c|}
\hline \multicolumn{6}{|c|}{ Single 5.0 in. Diameter Poly. Container in a Corner } \\
\hline \multicolumn{6}{|c|}{ for UO2F2 $+\mathrm{H} 2 \mathrm{O}$, Uranium Density $=3.2 \mathrm{gU} / \mathrm{cc}$} \\
\hline Case & $\mathrm{H} / \mathrm{U}$ & Keff & K sig & $2^{*} \mathrm{~K}$ sig & Keff $+2^{\star} K$ sig \\
\hline cpb01n-c & 0.5 & 0.6713 & 0.0043 & 0.0086 & 0.6799 \\
\hline cpb02n-c & 1 & 0.7092 & 0.0044 & 0.0088 & 0.718 \\
\hline $\mathrm{cpb03n-c}$ & 2 & 0.7606 & 0.0046 & 0.0092 & 0.7698 \\
\hline cpb04n-c & 3 & 0.8228 & 0.0048 & 0.0096 & 0.8324 \\
\hline cpb05n-c & 3.99 & 0.8811 & 0.0044 & 0.0088 & 0.8899 \\
\hline cpb06n-c & 4 & 0.8949 & 0.0051 & 0.0102 & 0.9051 \\
\hline cpb07n-c & 5 & 0.8911 & 0.005 & 0.01 & 0.9011 \\
\hline cpb08n-c & 10 & 0.9006 & 0.0045 & 0.009 & 0.9096 \\
\hline cpb09n-c & 15 & 0.9045 & 0.0053 & 0.0106 & 0.9151 \\
\hline cpb10n-c & 20 & 0.909 & 0.0055 & 0.011 & 0.92 \\
\hline$c p b 11 n-c$ & 25 & 0.9097 & 0.0056 & 0.0112 & 0.9209 \\
\hline cpb12n-c & 50 & 0.9103 & 0.0053 & 0.0106 & 0.9209 \\
\hline cpb13n-c & 100 & 0.8792 & 0.0058 & 0.0116 & 0.8908 \\
\hline cpb14n-c & 150 & 0.8238 & 0.0054 & 0.0108 & 0.8346 \\
\hline cpb15n-c & 200 & 0.8014 & 0.0051 & 0.0102 & 0.8116 \\
\hline
\end{tabular}


Figure 4.4.1-3. Two 5.00" ID $~ 24$ " Tall Cylinders of UO ${ }_{2} F_{2}$ Next to a Wall $100 \%$ onrichment, $24^{\prime \prime}$ conereto foor, $12^{\prime \prime}$ conereto wall and collhg, -100 kg water rofloctor cinalattog a parsox

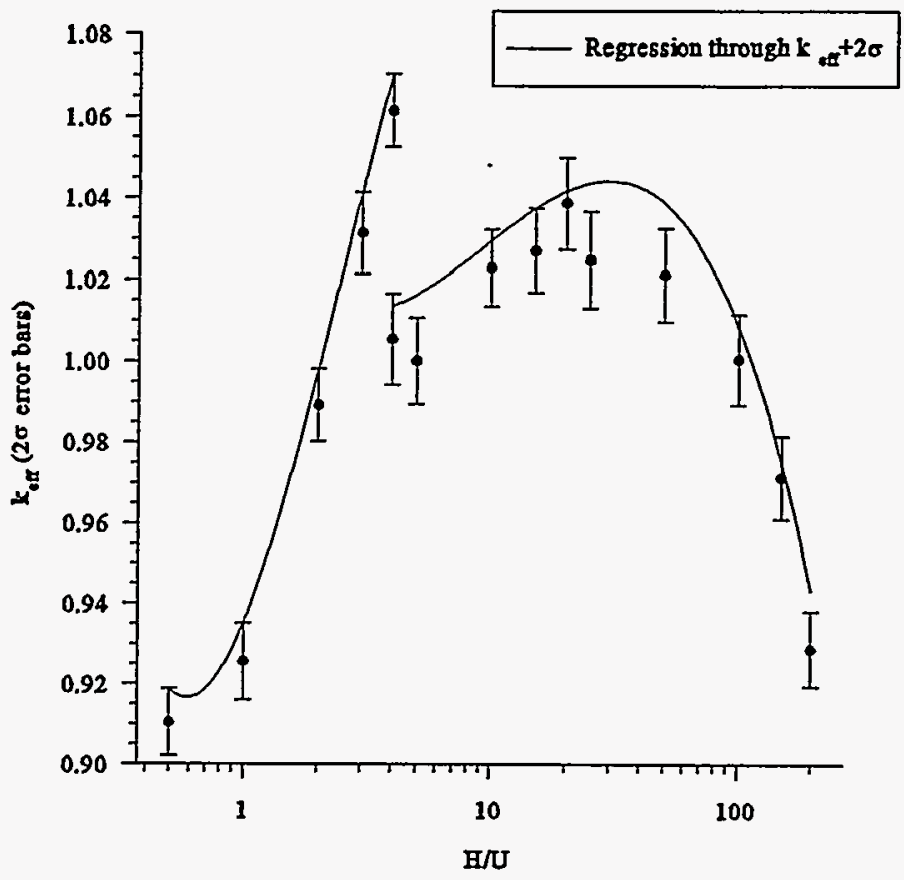

Two 5.0 in. Diameter Poly. Containers Adjacent to Concrete Wall for $100 \%$ Enriched UO2F2+ 120

\begin{tabular}{|c|c|c|c|c|c|}
\hline \hline Case & H/U & Keff & K sig & $2^{\star}$ K_sig & Keff $+2^{*}$ K sig \\
\hline npb001n.o & 0.5 & 0.9104 & 0.0042 & 0.0084 & 0.9188 \\
\hline npb002n.o & 1 & 0.9257 & 0.0048 & 0.0096 & 0.9353 \\
\hline npb003n.o & 2 & 0.9891 & 0.0045 & 0.009 & 0.9981 \\
\hline npb004n.o & 3 & 1.0313 & 0.005 & 0.01 & 1.0413 \\
\hline npb005n.o & 3.99 & 1.0614 & 0.0044 & 0.0088 & 1.0702 \\
\hline npb006n.o & 4 & 1.0053 & 0.0056 & 0.0112 & 1.0165 \\
\hline npb007n.o & 5 & 1 & 0.0053 & 0.0106 & 1.0106 \\
\hline npb008n.o & 10 & 1.0228 & 0.0047 & 0.0094 & 1.0322 \\
\hline npb009n.o & 15 & 1.027 & 0.0052 & 0.0104 & 1.0374 \\
\hline npb010n.o & 20 & 1.0386 & 0.0056 & 0.0112 & 1.0498 \\
\hline npb011n.o & 25 & 1.0247 & 0.0059 & 0.0118 & 1.0365 \\
\hline npb012n.o & 50 & 1.021 & 0.0057 & 0.0114 & 1.0324 \\
\hline npb013n.o & 100 & 1.0003 & 0.0056 & 0.0112 & 1.0115 \\
\hline npb014n.o & 150 & 0.9711 & 0.0051 & 0.0102 & 0.9813 \\
\hline npb015n.o & 200 & 0.9288 & 0.0047 & 0.0094 & 0.9382 \\
\hline
\end{tabular}


Figure 4.4.1-4. Two 5.00" $\mathrm{I} \times 24$ " Tall Cylinders of $\mathrm{UO}_{2} \mathrm{~F}_{2}$ One Foot from a Wall $100 \%$ enrichment, $24 "$ concrete floor, 12" concrete wall and ceiling, $\sim 100 \mathrm{~kg}$ water reflector simulating a person

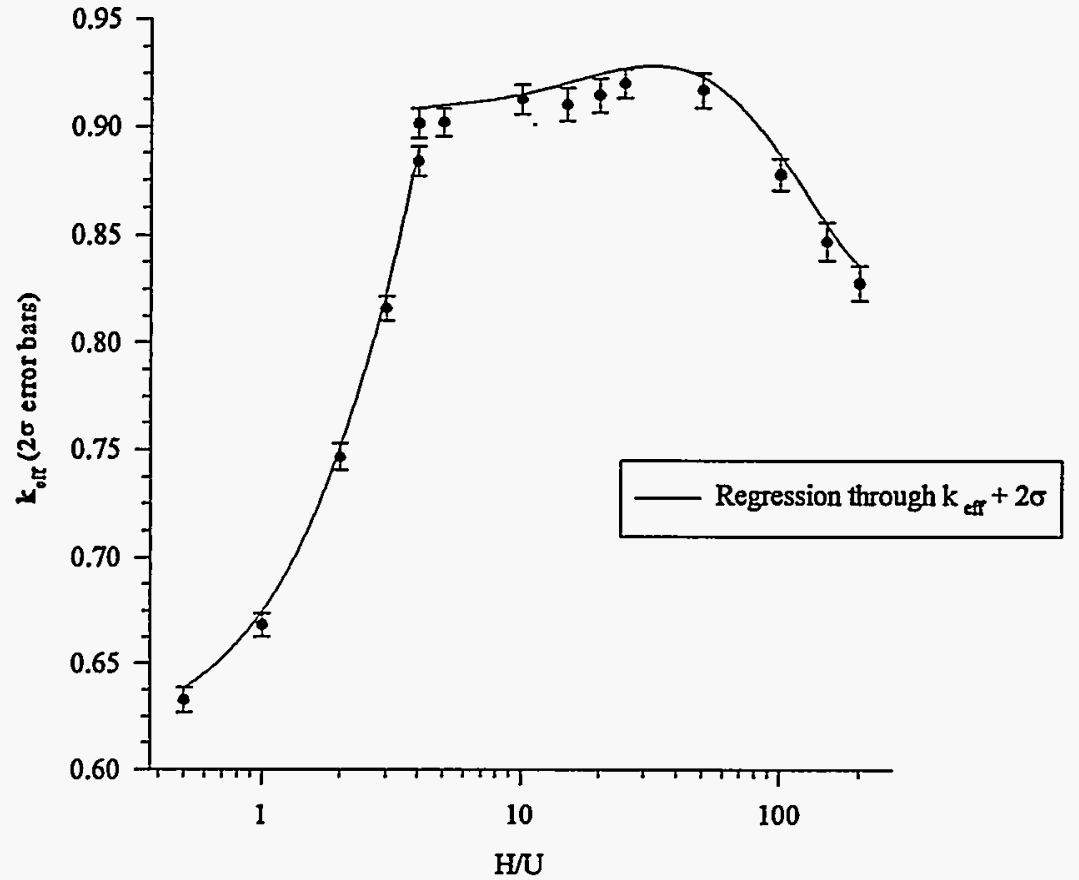

\begin{tabular}{|c|c|c|c|c|c|}
\hline \multicolumn{6}{|c|}{ Two 5.0 in. Diameter Poly. Containers 1 ' from Concrete Wall } \\
\hline \multicolumn{6}{|c|}{ for $100 \%$ Enriched UO2F $2+\mathrm{H} 2 \mathrm{O}$} \\
\hline Case & $\mathrm{H} / \mathrm{U}$ & Keff & K_sig & 2*K sig & Keff+2*K sig \\
\hline d12n-500.0p5 & 0.5 & 0.6327 & 0.00297 & 0.00594 & 0.63864 \\
\hline d12n-500.1 & 1 & 0.6684 & 0.00281 & 0.00562 & 0.67402 \\
\hline d12n-500.2 & 2 & 0.7465 & 0.00303 & 0.00606 & 0.75256 \\
\hline d12n-500.3 & 3 & 0.8158 & 0.00283 & 0.00566 & 0.82146 \\
\hline d12n-500.3p9 & 3.99 & 0.8835 & 0.00348 & 0.00696 & 0.89046 \\
\hline d12n-500.4 & 4 & 0.9013 & 0.00343 & 0.00686 & 0816 \\
\hline d12n-500.5 & 5 & 0.9018 & 0.00327 & 0.00654 & 0.90834 \\
\hline d12n-500.10 & 10 & 0.9125 & 0.00341 & 0.00682 & 0.91932 \\
\hline d12n-500.15 & 15 & 0.9099 & 0.00386 & 0.00772 & 0.91762 \\
\hline d12n-500.20 & 20 & 0.9143 & 0.00395 & 0.0079 & 0.9222 \\
\hline d12n-500.25 & 25 & 0.9197 & 0.00338 & 0.00676 & 0.92646 \\
\hline d12n-500.50 & 50 & 0.9164 & 0.00404 & 0.00808 & 0.92448 \\
\hline $\mathrm{d} 12 n-500.100$ & 100 & 0.8772 & 0.0036 & 0.0072 & 0.8844 \\
\hline d12n-500.150 & 150 & 0.8467 & 0.0043 & 0.0086 & 0.8553 \\
\hline d12n-500.200 & 200 & 0.8275 & 0.00397 & 0.00794 & 0.83544 \\
\hline
\end{tabular}


Figure 4.4.1-5. Single Fully Reflected 5.00" ID $\times 24$ " Tall Cylinder of UF and Oil $100 \%$ enrichment, 24 " concrete floor, 12" (minimum) water reflector on all other sides

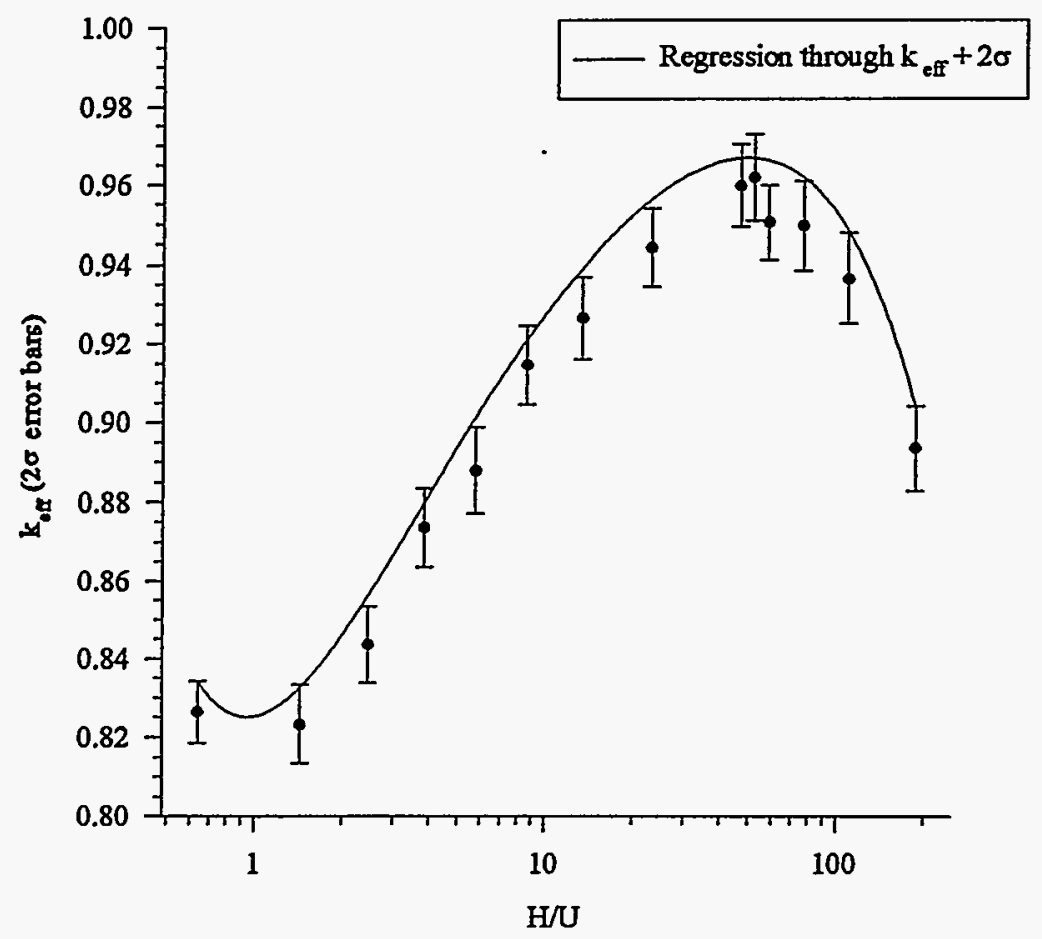

Single 5.0 in. Diameter Poly. Container w/ Full Reflection for $100 \%$ Enriched UF4+oil

\begin{tabular}{|c|c|c|c|c|c|}
\hline \hline Case & H/U & Keff & K sig & $2^{*}$ K_sig & Keff $+2^{*}$ K_sig \\
\hline fre001n.o & 0.64 & 0.8264 & 0.0039 & 0.0078 & 0.8342 \\
\hline fre002n.o & 1.44 & 0.8233 & 0.005 & 0.01 & 0.8333 \\
\hline fre003n.o & 2.47 & 0.8436 & 0.0049 & 0.0098 & 0.8534 \\
\hline fre004n.o & 3.84 & 0.8735 & 0.005 & 0.01 & 0.8835 \\
\hline fre005n.o & 5.76 & 0.8879 & 0.0054 & 0.0108 & 0.8987 \\
\hline fre006n.o & 8.64 & 0.9145 & 0.005 & 0.01 & 0.9245 \\
\hline fre007n.o & 13.4 & 0.9264 & 0.0052 & 0.0104 & 0.9368 \\
\hline fre008n.o & 23.1 & 0.9441 & 0.0048 & 0.0096 & 0.9537 \\
\hline fre009n.o & 46.6 & 0.9596 & 0.0052 & 0.0104 & 0.97 \\
\hline fre010n.o & 51.9 & 0.9617 & 0.0055 & 0.011 & 0.9727 \\
\hline fre011n.o & 58.2 & 0.9505 & 0.0046 & 0.0092 & 0.9597 \\
\hline fre012n.o & 76.6 & 0.9496 & 0.0056 & 0.0112 & 0.9608 \\
\hline fre013n.o & 109.5 & 0.9365 & 0.0057 & 0.0114 & 0.9479 \\
\hline fre014n.o & 186.4 & 0.8935 & 0.0053 & 0.0106 & 0.9041 \\
\hline
\end{tabular}


Figure 4.4.1-6. Single 5.00" ID $\times$ 24" Tall Cylinder of UF , and Oil in a Concrete Corner

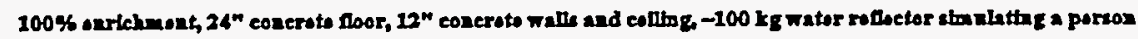

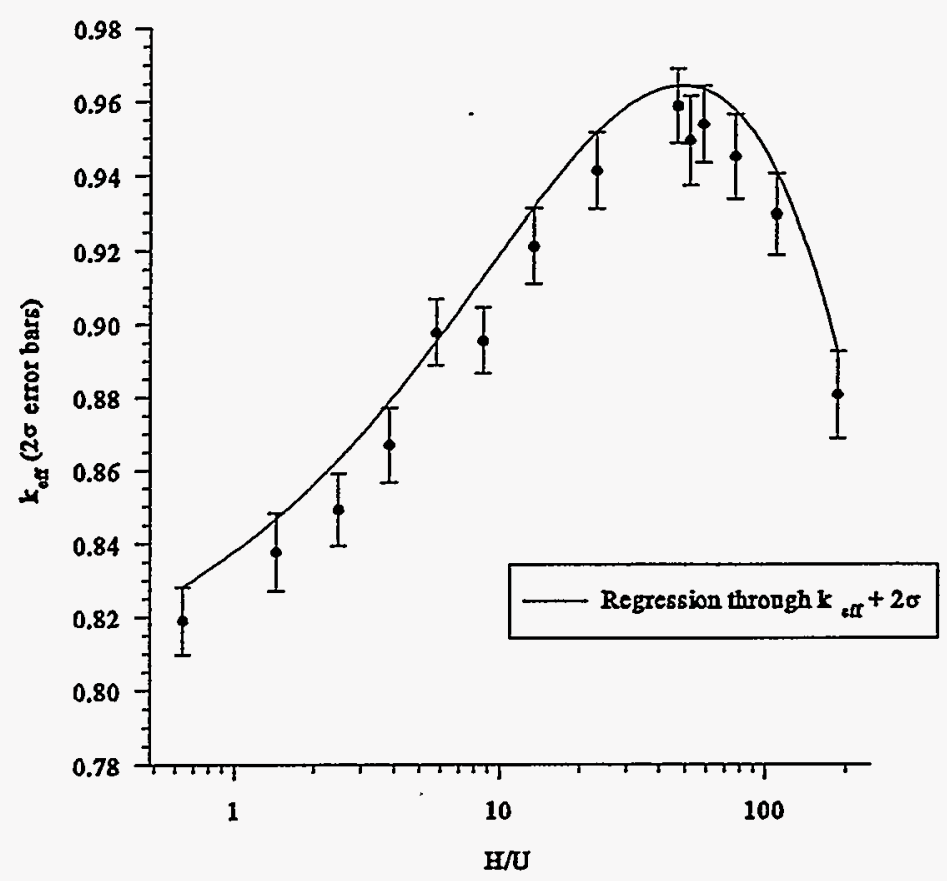

Single 5.0 in. Diameter Poly. Container in a Corner for $100 \%$ Enriched UF4+oil

\begin{tabular}{|c|c|c|c|c|c|}
\hline \hline Case & H/U & Keff & K sig & $2^{\star} \mathrm{K}$ sig & Keff $2^{\star} \mathrm{K}$ sig \\
\hline cpe001n.o & 0.64 & 0.819 & 0.0046 & 0.0092 & 0.8282 \\
\hline cpe002n.o & 1.44 & 0.8377 & 0.0053 & 0.0106 & 0.8483 \\
\hline cpe003n.o & 2.47 & 0.8491 & 0.0049 & 0.0098 & 0.8589 \\
\hline cpe004n.o & 3.84 & 0.8667 & 0.0051 & 0.0102 & 0.8769 \\
\hline cpe005n.o & 5.76 & 0.8975 & 0.0045 & 0.009 & 0.9065 \\
\hline cpe006n.o & 8.64 & 0.8952 & 0.0045 & 0.009 & 0.9042 \\
\hline cpe007n.o & 13.4 & 0.9208 & 0.0051 & 0.0102 & 0.931 \\
\hline cpe008n.o & 23.1 & 0.9409 & 0.0051 & 0.0102 & 0.9511 \\
\hline cpe009n.o & 46.6 & 0.9583 & 0.005 & 0.01 & 0.9683 \\
\hline cpe010n.o & 51.9 & 0.9489 & 0.006 & 0.012 & 0.9609 \\
\hline cpe011n.o & 58.2 & 0.9533 & 0.0052 & 0.0104 & 0.9637 \\
\hline cpe012n.o & 76.6 & 0.9445 & 0.0057 & 0.0114 & 0.9559 \\
\hline cpe013n.o & 109.5 & 0.9292 & 0.0054 & 0.0108 & 0.94 \\
\hline cpe014n.o & 186.4 & 0.8803 & 0.006 & 0.012 & 0.8923 \\
\hline
\end{tabular}


Figure 4.4.1-7. Two 5.00" $\mathrm{I} \times 24^{\prime \prime}$ Tall Cylinders of UF , and Oil Next to a Wall

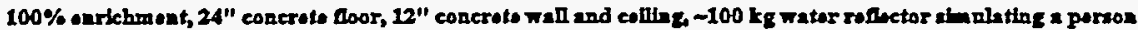

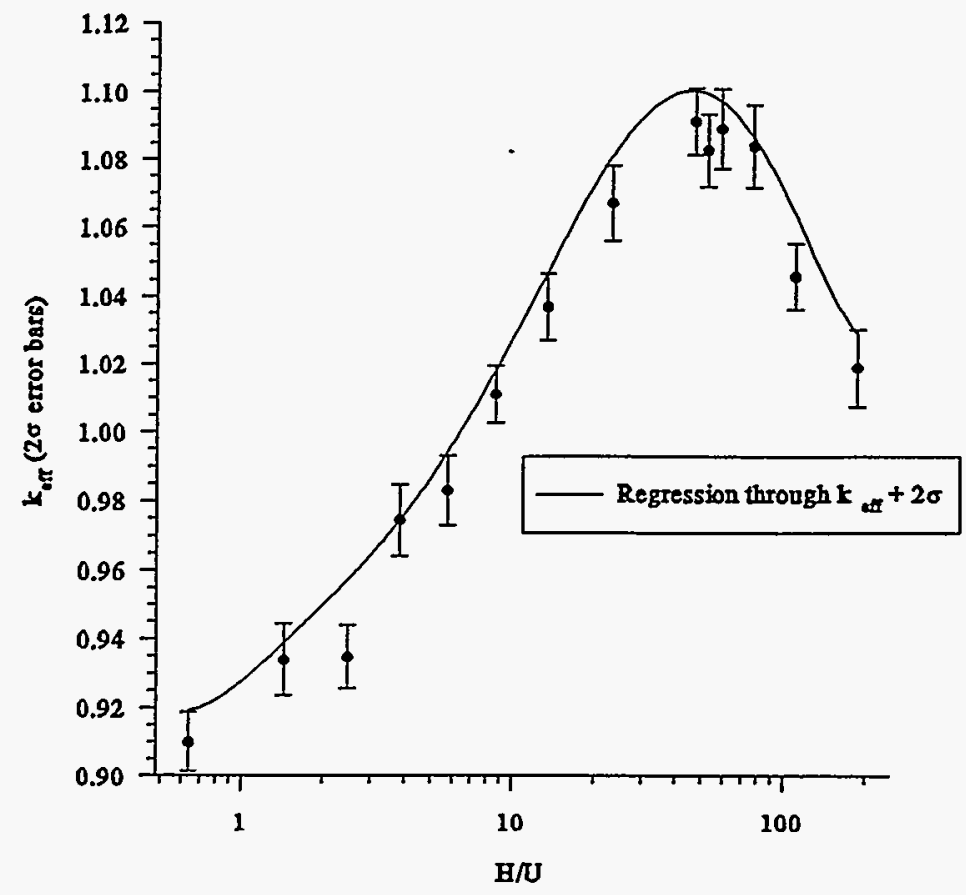

Two 5.0 in. Diameter Poly. Containers Adjacent to Concrete Wall for $100 \%$ Enriched UF4+oil

\begin{tabular}{|c|c|c|c|c|c|}
\hline \hline Case & HN & Keff & K sig & $2^{\star} \mathrm{K}$ sig & Keff+2* K sig \\
\hline \hline npe001n.o & 0.64 & 0.9099 & 0.0044 & 0.0088 & 0.9187 \\
\hline npe002n.o & 1.44 & 0.9339 & 0.0052 & 0.0104 & 0.9443 \\
\hline npe003n.o & 2.47 & 0.9346 & 0.0046 & 0.0092 & 0.9438 \\
\hline npe004n.o & 3.84 & 0.9743 & 0.0051 & 0.0102 & 0.9845 \\
\hline npe005n.o & 5.76 & 0.9829 & 0.005 & 0.01 & 0.9929 \\
\hline npe006n.o & 8.64 & 1.0108 & 0.0042 & 0.0084 & 1.0192 \\
\hline npe007n.o & 13.4 & 1.0366 & 0.0049 & 0.0098 & 1.0464 \\
\hline npe008n.o & 23.1 & 1.0667 & 0.0055 & 0.011 & 1.0777 \\
\hline npe009n.o & 46.6 & 1.0908 & 0.0049 & 0.0098 & 1.1006 \\
\hline npe010n.o & 51.9 & 1.0822 & 0.0054 & 0.0108 & 1.093 \\
\hline npe011n.o & 58.2 & 1.0886 & 0.0059 & 0.0118 & 1.1004 \\
\hline npe012n.o & 76.6 & 1.0834 & 0.0061 & 0.0122 & 1.0956 \\
\hline npe013n.o & 109.5 & 1.0455 & 0.0048 & 0.0096 & 1.0551 \\
\hline npe014n.o & 186.4 & 1.0185 & 0.0057 & 0.0114 & 1.0299 \\
\hline
\end{tabular}


POEF-SH-30

Figure 4.4.1-8. Two 5.00" ID × 24" Tall Cylinders of UF and Oil One Foot from 2 Wall

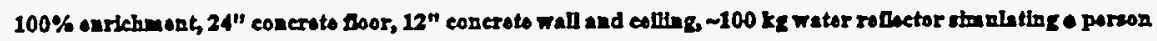

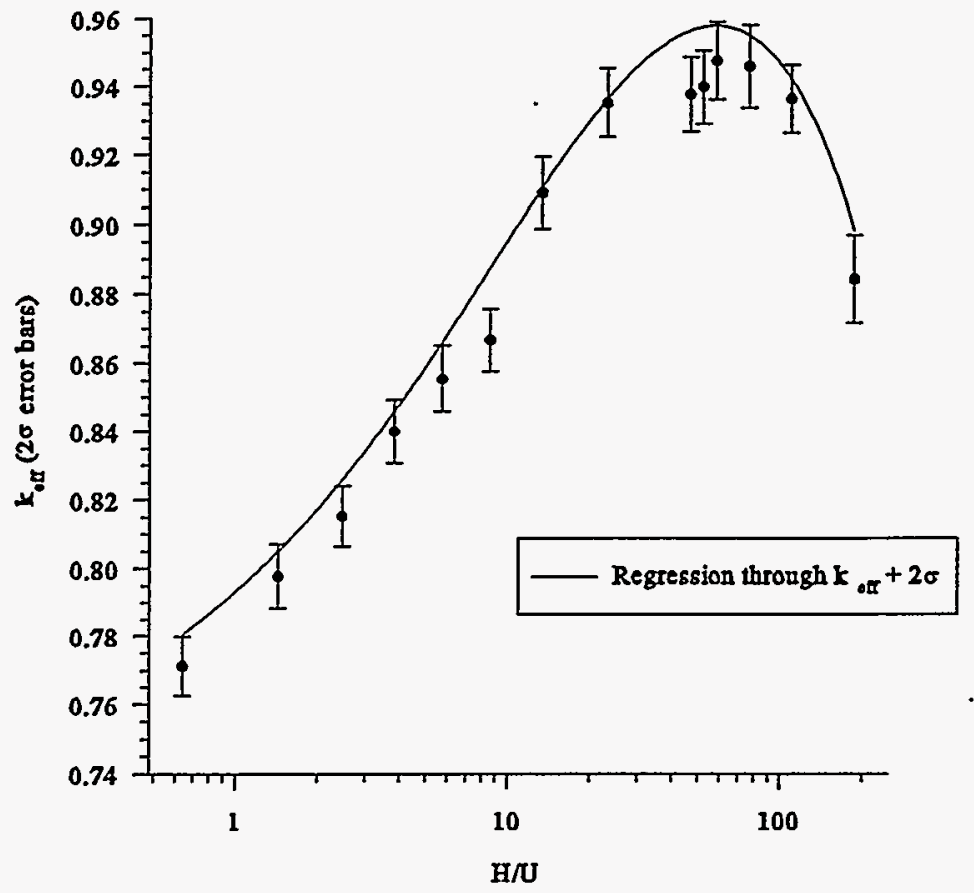

\begin{tabular}{|c|c|c|c|c|c|}
\hline \multicolumn{6}{|c|}{ Two 5.0 in. Diameter Poly. Containers 1 ' from Concrete Wall } \\
\hline \multicolumn{6}{|c|}{ for $100 \%$ Enriched UF4+oil } \\
\hline Case & $\mathrm{H} / \mathrm{U}$ & Keff & Ksig & $2^{\star *}$ K_sig & Keff $+22^{\star}$ K sig \\
\hline ape001n.o & 0.64 & 0.7712 & 0.0043 & 0.0086 & 0.7798 \\
\hline ape002n.o & 1.44 & 0.7978 & 0.0047 & 0.0094 & 0.8072 \\
\hline ape003n.o & 2.47 & 0.8152 & 0.0044 & 0.0088 & 0.824 \\
\hline ape004n.o & 3.84 & 0.8398 & 0.0046 & 0.0092 & 0.849 \\
\hline ape005n.o & 5.76 & 0.8552 & 0.0048 & 0.0096 & 0.8648 \\
\hline ape006n.o & 8.64 & 0.8664 & 0.0045 & 0.009 & 0.8754 \\
\hline ape007n.o & 13.4 & 0.9087 & 0.0052 & 0.0104 & 0.9191 \\
\hline ape008n.o & 23.1 & 0.9348 & 0.005 & 0.01 & 0.9448 \\
\hline ape009n.o & 46.6 & 0.9372 & 0.0054 & 0.0108 & 0.948 \\
\hline ape010n.o & 51.9 & 0.9393 & 0.0054 & 0.0108 & 0.9501 \\
\hline ape011n.o & 58.2 & 0.947 & 0.0057 & 0.0114 & 0.9584 \\
\hline ape $012 n .0$ & 76.6 & 0.9452 & 0.006 & 0.012 & 0.9572 \\
\hline ape013n.o & 109.5 & 0.9357 & 0.0049 & 0.0098 & 0.9455 \\
\hline ape014n.o & 186.4 & 0.8838 & 0.0063 & 0.0126 & 0.8964 \\
\hline
\end{tabular}


Figure 4.4.1-9. An Array of 5.00" ID $\times 24$ " Tall Cylinders of UO ${ }_{2} F_{2}$

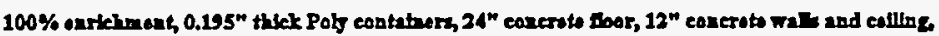
6' spectug botween row sets, 23 " spaciog butwean contaloner, 2 ' spacheg trone walls

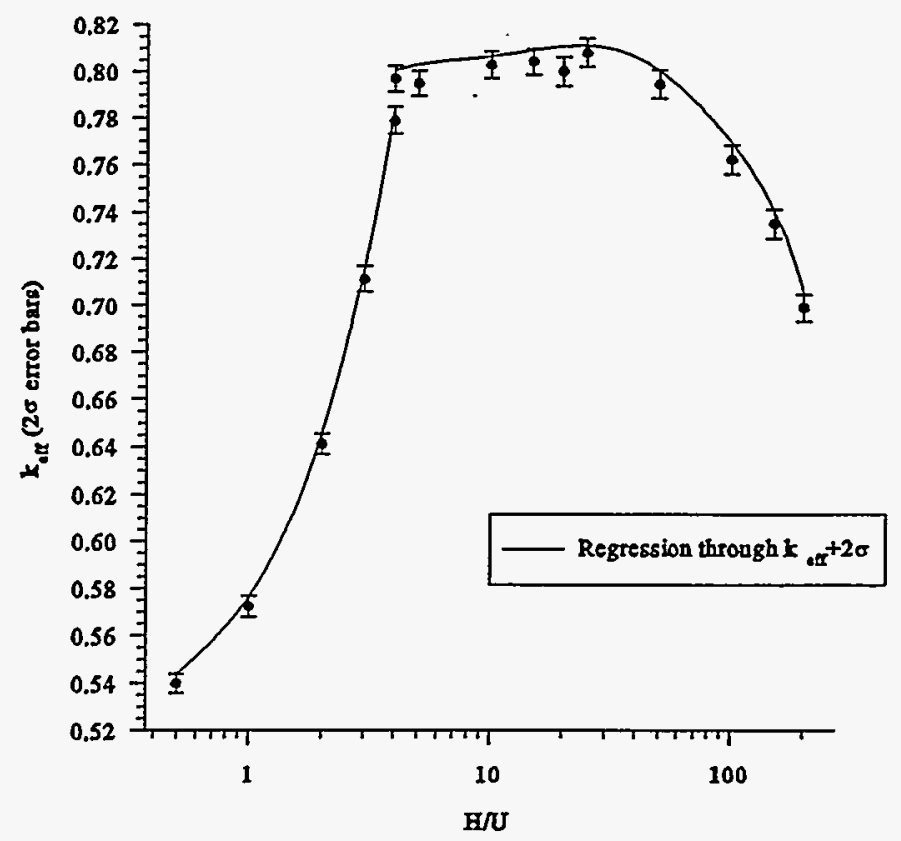

\begin{tabular}{|c|c|c|c|c|c|}
\hline \multicolumn{6}{|c|}{ An Array of 5.00-in. Poly. Containers of UO2F2 For 100\% Enrichment } \\
\hline \hline Case & H/U & Keff & K sig & $2^{\star}$ K_sig & Keff+2*K_sig \\
\hline \hline arrb01n-c.o & 0.5 & 0.5399 & 0.002 & $0: 004$ & 0.5439 \\
\hline arrb02n-c.o & 1 & 0.5724 & 0.0022 & 0.0044 & 0.5768 \\
\hline arrb03n-c.o & 2 & 0.6409 & 0.0022 & 0.0044 & 0.6453 \\
\hline arrb04n-c.o & 3 & 0.7112 & 0.0028 & 0.0056 & 0.7168 \\
\hline arrb05n-c.o & 3.99 & 0.7786 & 0.0029 & 0.0058 & 0.7844 \\
\hline \hline arrb06n-c.o & 4 & 0.7965 & 0.0027 & 0.0054 & 0.8019 \\
\hline arrb07n-c.o & 5 & 0.7945 & 0.0026 & 0.0052 & 0.7997 \\
\hline arrb08n-c.o & 10 & 0.8024 & 0.0028 & 0.0056 & 0.808 \\
\hline arrb09n-c.o & 15 & 0.8036 & 0.0028 & 0.0056 & 0.8092 \\
\hline arrb10n-c.o & 20 & 0.7994 & 0.0031 & 0.0062 & 0.8056 \\
\hline arrb11n-c.o & 25 & 0.8074 & 0.003 & 0.006 & 0.8134 \\
\hline arrb12n-c.o & 50 & 0.7938 & 0.003 & 0.006 & 0.7998 \\
\hline arrb13n-c.o & 100 & 0.7614 & 0.0031 & 0.0062 & 0.7676 \\
\hline arrb14n-c.o & 150 & 0.7345 & 0.0031 & 0.0062 & 0.7407 \\
\hline arrb15n-c.o & 200 & 0.6985 & 0.0029 & 0.0058 & 0.7043 \\
\hline
\end{tabular}


Figure 4.4.1-10. An Array of 5.00" $\mathrm{D} \times 24$ " Tall Cylinders of UF , Oil

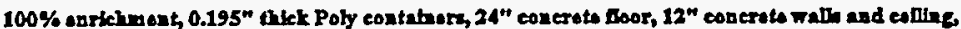

6 spacheg botween row setz, $23^{\mathrm{N}}$ spactag botwoen contuhers, 24 spaciag trom walls

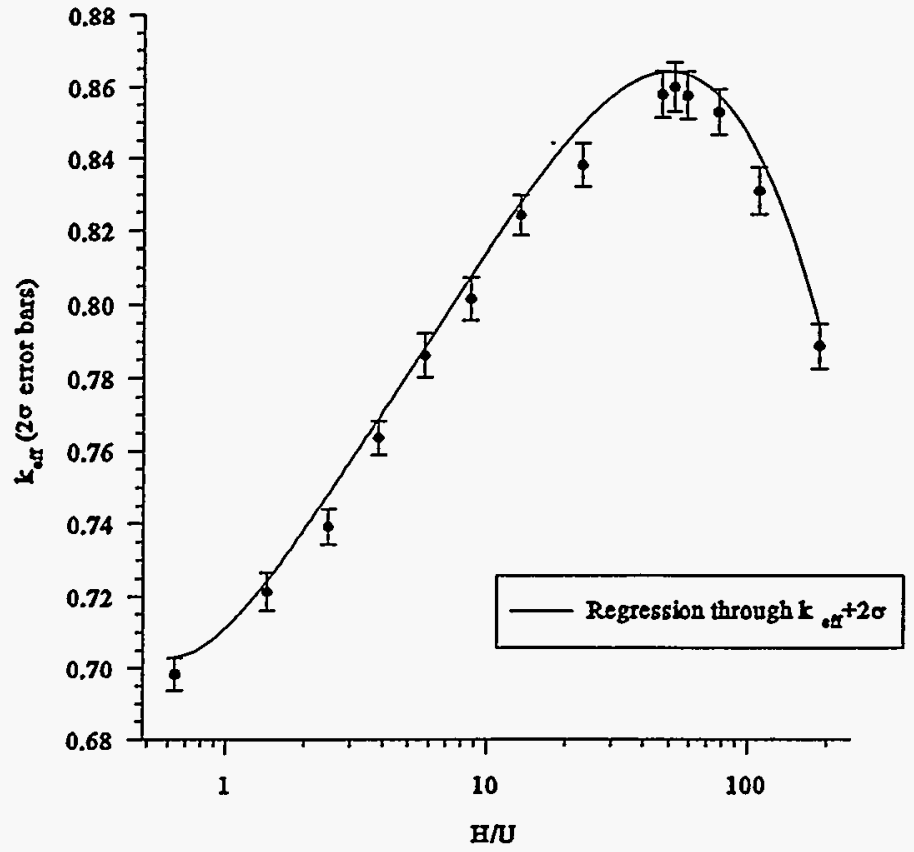

An Array of 5.0 -in. Poly Cylinders of UF4+Oil For $100 \%$ Enrichment

\begin{tabular}{|c|c|c|c|c|c|}
\hline Case & H/U & Keff & K sig & $2^{*} \mathrm{~K}$ sig & Keff $+2^{*} \mathrm{~K}$ sig \\
\hline arre01n-c.o & 0.64 & 0.6983 & 0.0023 & 0.0046 & 0.7029 \\
\hline arre02n-c.o & 1.44 & 0.7213 & 0.0027 & 0.0054 & 0.7267 \\
\hline arre03n-c.o & 2.47 & 0.7391 & 0.0024 & 0.0048 & 0.7439 \\
\hline arre04n-c.o & 3.84 & 0.7635 & 0.0024 & 0.0048 & 0.7683 \\
\hline arre05n-c.o & 5.76 & 0.786 & 0.003 & 0.006 & 0.792 \\
\hline arre06n-c.o & 8.64 & 0.8012 & 0.0029 & 0.0058 & 0.807 \\
\hline arre07n-c.o & 13.4 & 0.8239 & 0.0028 & 0.0056 & 0.8295 \\
\hline arre08n-c.o & 23.1 & 0.8377 & 0.003 & 0.006 & 0.8437 \\
\hline arre09n-c.o & 46.6 & 0.8574 & 0.0032 & 0.0064 & 0.8638 \\
\hline arre10n-c.o & 51.9 & 0.8594 & 0.0034 & 0.0068 & 0.8662 \\
\hline arre11n-c.o & 58.2 & 0.857 & 0.0033 & 0.0066 & 0.8636 \\
\hline arre12n-c.o & 76.6 & 0.8524 & 0.0032 & 0.0064 & 0.8588 \\
\hline arre13n-c.o & 109.5 & 0.8304 & 0.0033 & 0.0066 & 0.837 \\
\hline arre14n-c.o & 186.4 & 0.7882 & 0.0031 & 0.0062 & 0.7944 \\
\hline
\end{tabular}


Figure 4.4.1-11. The Effects of Interstitial Water Volume Fraction on $k$ or

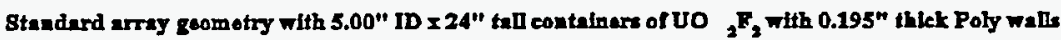

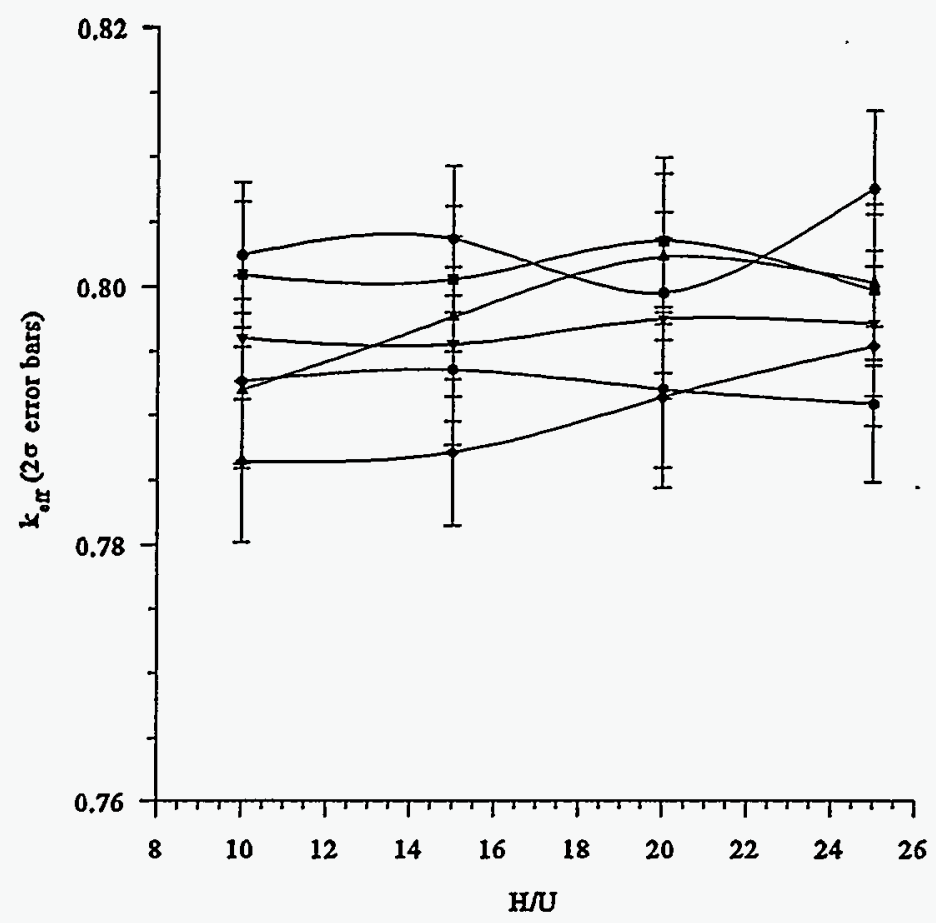

- W Water Volume Fraction $=0.0$

$\rightarrow$ Water Volume Fraction $=0.001$

$\longrightarrow$ Water Volume Fraction $=0.005$

$\longrightarrow$ Water Volume Fraction $=0.01$

$\rightarrow$ Water Volume Fraction $=0.05$

$\rightarrow$ Water Volume Fraction $=0.1$ 
Figure 4.4.1-11a. The Effects of Interstitial Water Volume Fraction on $\mathbf{k}_{\text {eff }}$ Standard array geometry with 5.00 " ID $\times 24$ " tall containers of $\mathrm{UO}_{2} \mathrm{~F}_{2}$ with 0.195 " thick Poly walls

\begin{tabular}{|c|c|c|c|c|c|}
\hline \multicolumn{4}{|c|}{ The Effects of Interstitial Water Volume Fraction for a Standard } & & \\
\hline \multicolumn{6}{|c|}{ Array of 5.00 -in. Diameter Containers of $\mathrm{UO} 2 \mathrm{~F} 2+\mathrm{H} 2 \mathrm{O}$} \\
\hline Case & $\mathrm{H} / \mathrm{U}$ & Keff & K_sig & $2 * K$ sig & Keff $+2^{*} \mathrm{~K}$ sig \\
\hline Interstitial & Water $=$ & 0 & & & \\
\hline arrb08n-c.o & 10 & 0.8024 & 0.0028 & 0.0056 & 0.808 \\
\hline arrb09n-c.o & 15 & 0.8036 & 0.0028 & 0.0056 & 0.8092 \\
\hline arrb10n-c.o & 20 & 0.7994 & 0.0031 & 0.0062 & 0.8056 \\
\hline amb11n-c.o & 25 & 0.8074 & 0.003 & 0.006 & 0.8134 \\
\hline Interstitial & Water $=$ & 0.001 & & & \\
\hline abw101n-c.o & 10 & 0.8009 & 0.0028 & 0.0056 & 0.8065 \\
\hline$a b w 102 n-c .0$ & 15 & 0.8005 & 0.0028 & 0.0056 & 0.8061 \\
\hline abw103n-c.o & 20 & 0.8034 & 0.0032 & 0.0064 & 0.8098 \\
\hline$a b w 104 n-c, 0$ & 25 & 0.7996 & 0.0029 & 0.0058 & 0.8054 \\
\hline Interstitial & Water $=$ & 0.005 & & & \\
\hline$a b w 201 n-c .0$ & 10 & 0.7919 & 0.003 & 0.006 & 0.7979 \\
\hline$a b w 202 n-c .0$ & 15 & 0.7976 & 0.0031 & 0.0052 & 0.8038 \\
\hline abw203n-c.o & 20 & 0.8022 & 0.0032 & 0.0064 & 0.8086 \\
\hline$a b w 204 n-c .0$ & 25 & 0.8002 & 0.003 & 0.006 & 0.8062 \\
\hline Interstitial & Water = & 0.01 & & & \\
\hline abw301n-c.o & 10 & 0.796 & 0.0024 & 0.0048 & 0.8008 \\
\hline abw302n-c.o & 15 & 0.7955 & 0.003 & 0.006 & 0.8015 \\
\hline$a b w 303 n-c .0$ & 20 & 0.7974 & 0.0031 & 0.0062 & 0.8036 \\
\hline abw304n-c.o & 25 & 0.797 & 0.0028 & 0.0056 & 0.8026 \\
\hline Interstitial & Water $=$ & 0.05 & & & \\
\hline abw401n-c.o & 10 & 0.7864 & 0.0031 & 0.0062 & 0.7926 \\
\hline abw402n-c.o & 15 & 0.7871 & 0.0028 & 0.0056 & 0.7927 \\
\hline abw403n-c.o & 20 & 0.7913 & 0.0035 & 0.007 & 0.7983 \\
\hline abw404n-c.o & 25 & 0.7953 & 0.0031 & 0.0062 & 0.8015 \\
\hline Interstitial & Water $=$ & 0.1 & & & \\
\hline abw501n-c.o & 10 & 0.7926 & 0.0032 & 0.0064 & 0.799 \\
\hline$a b w 502 n-c .0$ & 15 & 0.7935 & 0.0029 & 0.0058 & 0.7993 \\
\hline abw503n-c.o & 20 & 0.7919 & 0.003 & 0.006 & 0.7979 \\
\hline abw504n-c.o & 25 & 0.7908 & 0.003 & 0.006 & 0.7968 \\
\hline
\end{tabular}


Figure 4.4.1-12. An Array of 5.00" ID $\times 24$ " Tall Cylinders of $U O_{2} F_{2}$

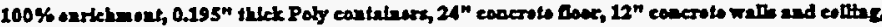

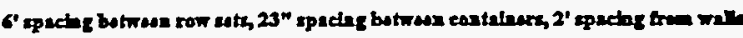

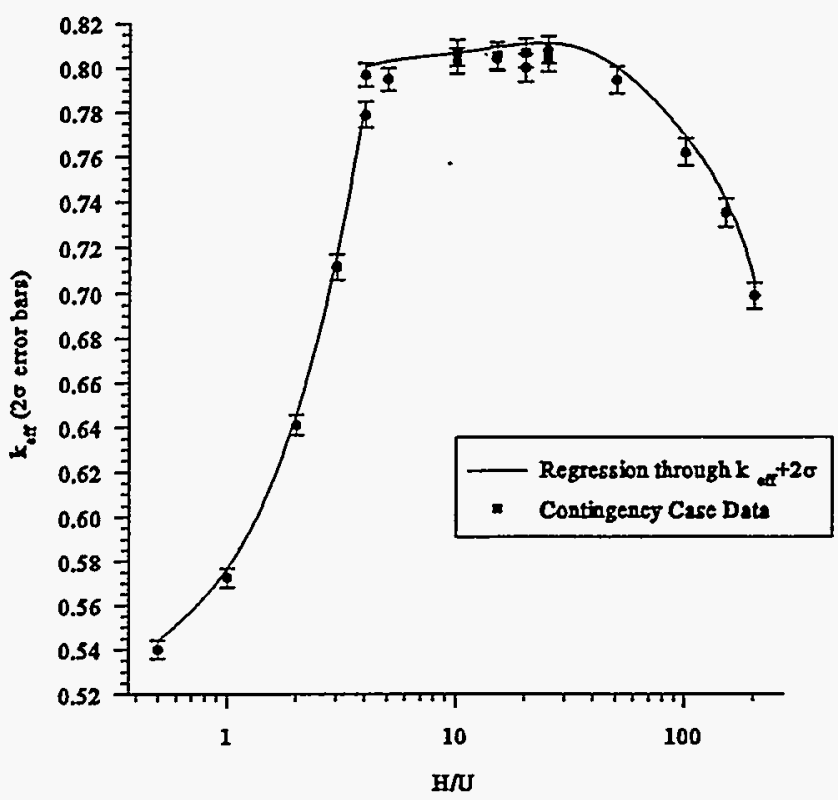

\begin{tabular}{|c|c|c|c|c|c|}
\hline \multicolumn{6}{|c|}{ An Array of 5.00-in. Containers of UO2F2 (Contingency Data) } \\
\hline \multicolumn{6}{|c|}{ For $100 \%$ Enrichment } \\
\hline & H/u & Keif & $x a y$ & & \\
\hline arrb01n-c.o & 0.5 & 0.5399 & 0.002 & 0.004 & 0.5439 \\
\hline amb02n-c.o & 1 & 0.5724 & 0.0022 & 0.0044 & 0.5768 \\
\hline arrb03n-c.o & 2 & 0.6409 & 0.0022 & 0.0044 & 0.6453 \\
\hline arrb04n-c.o & 3 & 0.7112 & 0.0028 & 0.0056 & 0.7168 \\
\hline arrb05n-c.o & 3.99 & 0.7786 & 0.0029 & 0.0058 & 0.7844 \\
\hline amb06n-c.o & 4 & 0.7965 & 0.0027 & 0.0054 & 0.8019 \\
\hline amb07n-c.o & 5 & 0.7945 & 0.0026 & 0.0052 & 0.7997 \\
\hline arrb08n-c.o & 10 & 0.8024 & 0.0028 & 0.0056 & 0.808 \\
\hline arrbogn-c.o & 15 & 0.8036 & 0.0028 & 0.0056 & 0.8092 \\
\hline arrb10n-c.o & 20 & 0.7994 & 0.0031 & 0.0062 & 0.8056 \\
\hline arrb11n-c.o & 25 & 0.8074 & 0.003 & 0.006 & 0.8134 \\
\hline arrb12n-c.o & 50 & 0.7938 & 0.003 & 0.006 & 0.7998 \\
\hline arrb13n-c.o & 100 & 0.7614 & 0.0031 & 0.0062 & 0.7676 \\
\hline arrb14n-c.o & 150 & 0.7345 & 0.0031 & 0.0062 & 0.7407 \\
\hline arrb15n-c.o & 200 & 0.6985 & 0.0029 & 0.0058 & 0.7043 \\
\hline Case & $H / U$ & Keff & K sig & $2^{*} \mathrm{~K}$ sig & Keff $+2^{*} \mathrm{~K}$ sig \\
\hline arben $08-c .0$ & 10 & 0.8061 & 0.0029 & 0.0058 & 0.8119 \\
\hline arben09-c.o & 15 & 0.8048 & 0.003 & 0.006 & 0.8108 \\
\hline arben10-c.o & 20 & 0.8061 & 0.0032 & 0.0064 & 0.8125 \\
\hline arben11-c.o & 25 & 0.8039 & 0.0031 & 0.0062 & 0.8101 \\
\hline
\end{tabular}


POEF-SH-30

\subsubsection{0\% Enriched $\mathrm{UO}_{2}^{\prime} \mathrm{F}_{2}+\mathrm{H}_{2} \mathrm{O}$ and $\mathrm{UF}_{4}+\mathrm{Oil}$}

Each of the five standard contingency cases was repeated for both material types. Table 4.4.2-0 gives the figure and table numbers that contain the results data for this container with various geometry models and fuel materials. The geometry model designators are explained in Table 4.1-1.

The three standard array cases were not analyzed because the $100 \%$ enriched standard array cases fell within the safety criterion. Container poly thickness was only considered for the TACW and TSCW $\mathrm{UF}_{4}$ toil cases because these results bound the $\mathrm{UO}_{2} \mathrm{~F}_{2}+\mathrm{H}_{2} \mathrm{O}$ cases.

Table 4.4.2-0. Summary of 5.0-inch Bottle References for $10 \%$ Enrichment

\begin{tabular}{|c|c|c||}
\hline Geometry Model & Material & Figure/Table Number \\
\hline \hline SFR & $\mathrm{UO}_{2} \mathrm{~F}_{2}+\mathrm{H}_{2} \mathrm{O}$ & $4.4 .2-1$ \\
\hline $\mathrm{SCC}$ & $\mathrm{UO}_{2} \mathrm{~F}_{2}+\mathrm{H}_{2} \mathrm{O}$ & $4.4 .2-2$ \\
\hline TACW & $\mathrm{UO}_{2} \mathrm{~F}_{2}+\mathrm{H}_{2} \mathrm{O}$ & $4.4 .2-3$ \\
\hline TSCW & $\mathrm{UO}_{2} \mathrm{~F}_{2}+\mathrm{H}_{2} \mathrm{O}$ & $4.4 .2-4$ \\
\hline SFR & $\mathrm{UF}_{4}+$ oil & $4.4 .2-5$ \\
\hline SCC & $\mathrm{UF}_{4}+$ oil & $4.4 .2-6$ \\
\hline TACW & $\mathrm{UF}_{4}+$ oil & $4.4 .2-7$ \\
\hline TSCW & $\mathrm{UF}_{4}+$ oil & $4.4 .2-8$ \\
\hline
\end{tabular}


Figure 4.4.2-1. Single Fally Reflected 5.00" ID $\times 24$ " Tall Cylinder of UO ${ }_{2} F_{2}$

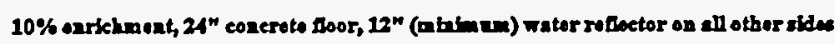

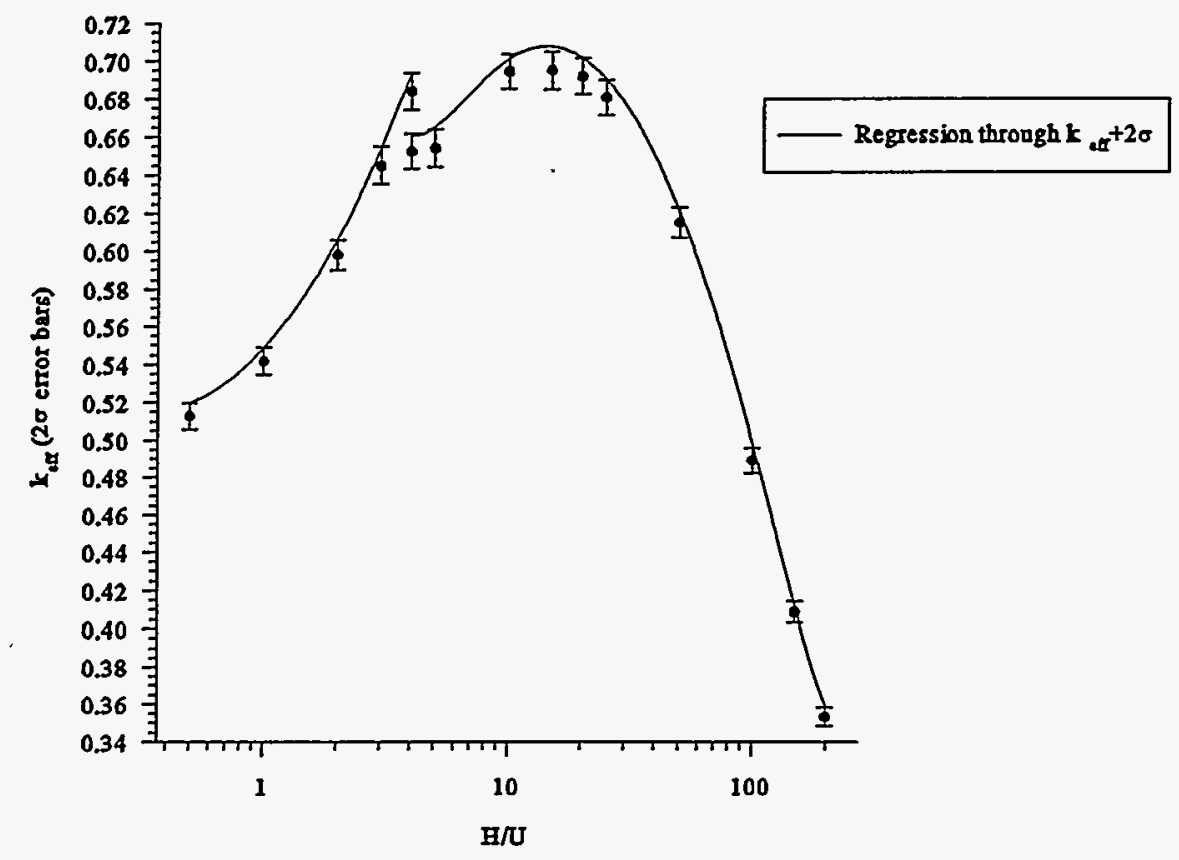

\begin{tabular}{|c|c|c|c|c|c|}
\hline \multicolumn{6}{|c|}{ Single 5.0 in. Diameter Poly. Container w/ Full Reflection } \\
\hline \multicolumn{6}{|c|}{ for $10 \%$ Enriched UO2F $+\mathrm{H} 2 \mathrm{O}$} \\
\hline Case & $\mathrm{H} / \mathrm{U}$ & Keff & K_sig & 2*K sig & Keff $+2 *$ K sig \\
\hline frv001n.o & 0.5 & 0.5127 & 0.0034 & 0.0068 & 0.5195 \\
\hline frv002n.o & 1 & 0.5417 & 0.0036 & 0.0072 & 0.5489 \\
\hline frv003n.o & 2 & 0.5978 & 0.004 & 0.008 & 0.6058 \\
\hline frv004n.o & 3 & 0.6446 & 0.0049 & 0.0098 & 0.6544 \\
\hline frv005n.o & 3.99 & 0.6836 & 0.0049 & 0.0098 & 0.6934 \\
\hline frv006n.o & 4 & 0.652 & 0.0045 & 0.009 & 0.661 \\
\hline frv007n.o & 5 & 0.6537 & 0.0048 & 0.0096 & 0.6633 \\
\hline frv008n.o & 10 & 0.6938 & 0.0046 & 0.0092 & 0.703 \\
\hline frv009n.o & 15 & 0.6944 & 0.005 & 0.01 & 0.7044 \\
\hline frv010n.o & 20 & 0.6912 & 0.0047 & 0.0094 & 0.7006 \\
\hline frv011n.o & 25 & 0.68 & 0.0047 & 0.0094 & 0.6894 \\
\hline frv012n.o & 50 & 0.6144 & 0.004 & 0.008 & 0.6224 \\
\hline frv013n.o & 100 & 0.4887 & 0.0035 & 0.007 & 0.4957 \\
\hline frv014n.o & 150 & 0.4086 & 0.0027 & 0.0054 & 0.414 \\
\hline frv015n.o & 200 & 0.3533 & 0.0024 & 0.0048 & 0.3581 \\
\hline
\end{tabular}


Figure 4.4.2-2. Single 5.00" ID $\times 24$ " Tall Cylinder of UO ${ }_{2} F_{2}$ in 2 Concrete Corner

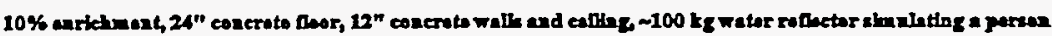

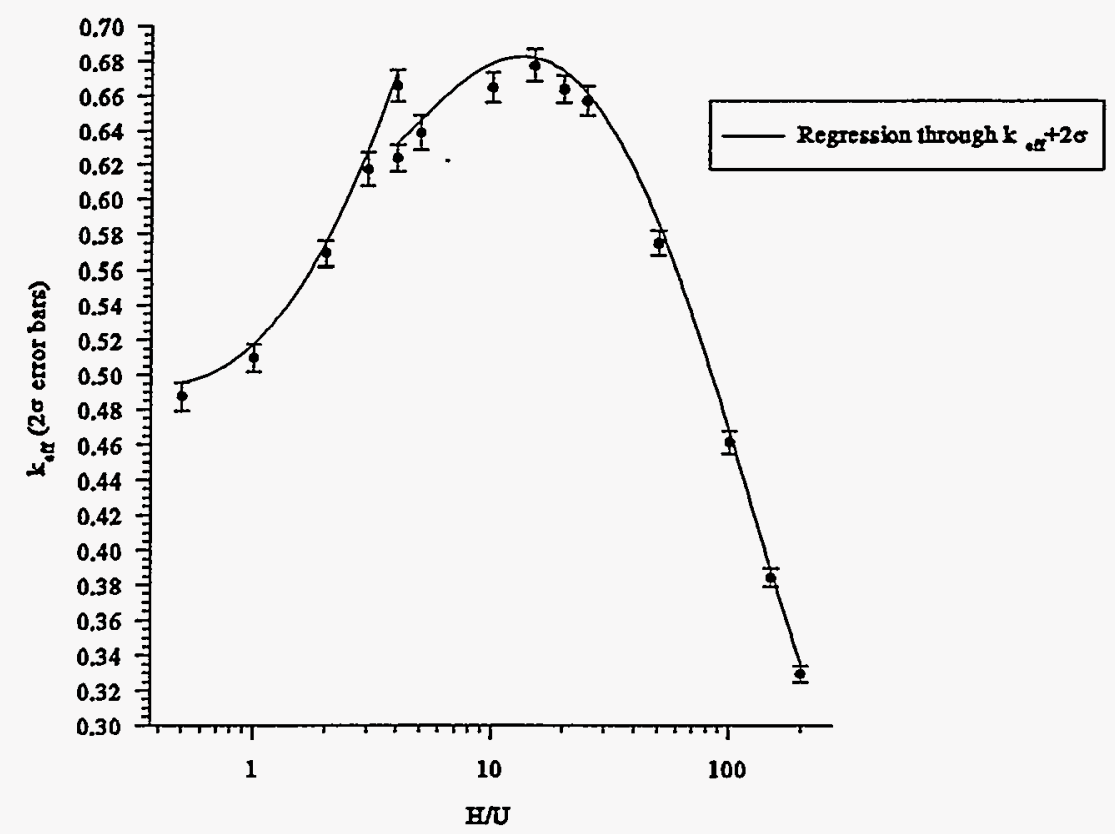

\begin{tabular}{|c|c|c|c|c|c|}
\hline \multicolumn{6}{|c|}{ Single 5.0 in. Diameter Poly. Container in a Corner } \\
\hline \multicolumn{6}{|c|}{ for $10 \%$ Enriched UO2F $2+\mathrm{H} 2 \mathrm{O}$} \\
\hline Case & $\mathrm{H} / \mathrm{U}$ & Keff & K_sig & $2^{*} \mathrm{~K}$ sig & Keff $+2^{*} \mathrm{~K}$ sig \\
\hline cpv001n.o & 0.5 & 0.4873 & 0.0041 & 0.0082 & 0.4955 \\
\hline cpv002n.o & 1 & 0.5095 & 0.0039 & 0.0078 & 0.5173 \\
\hline cpv003n.o & 2 & 0.5689 & 0.0036 & 0.0072 & 0.5761 \\
\hline cpv004n.o & 3 & 0.6168 & 0.0049 & 0.0098 & 0.6266 \\
\hline cpv005n.o & 3.99 & 0.6652 & 0.0046 & 0.0092 & 0.6744 \\
\hline cpv006n.o & 4 & 0.6233 & 0.0039 & 0.0078 & 0.6311 \\
\hline cpv007n.o & 5 & 0.6382 & 0.005 & 0.01 & 0.6482 \\
\hline cpv008n.o & 10 & 0.6642 & 0.0043 & 0.0086 & 0.6728 \\
\hline cpv009n.o & 15 & 0.6769 & 0.0046 & 0.0092 & 0.6861 \\
\hline cpv010n.o & 20 & 0.663 & 0.0039 & 0.0078 & 0.6708 \\
\hline cpv011n.o & 25 & 0.6563 & 0.0042 & 0.0084 & 0.6647 \\
\hline cpv012n.o & 50 & 0.5741 & 0.0035 & 0.007 & 0.5811 \\
\hline cpv013n.o & 100 & 0.4609 & 0.0033 & 0.0066 & 0.4675 \\
\hline cpv014n.o & 150 & 0.3844 & 0.0027 & 0.0054 & 0.3898 \\
\hline cpv015n.o & 200 & 0.3294 & 0.0023 & 0.0046 & 0.334 \\
\hline
\end{tabular}


Figure 4.4.2-3. Two 5.00" ID $~ 224 "$ Tall Cylinders of UO ${ }_{2} \mathrm{~F}_{2}$ Next to a Wall

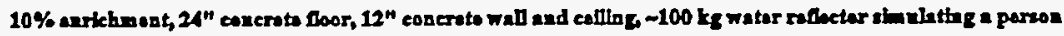

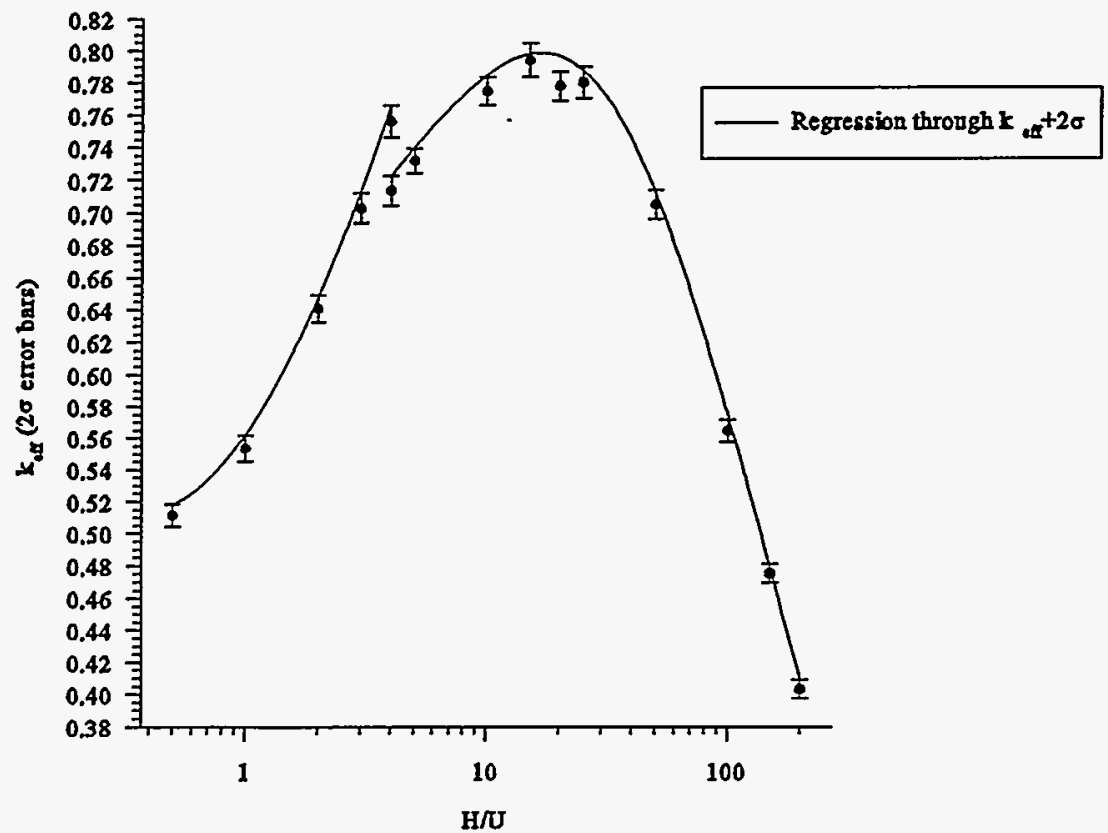

\begin{tabular}{|c|c|c|c|c|c|}
\hline \multicolumn{6}{|c|}{ Two 5.0 in. Diameter Poly. Containers Adjacent to Concrete Wall } \\
\hline \multicolumn{6}{|c|}{ for $10 \%$ Enriched UO2F $2+\mathrm{H} 2 \mathrm{O}$} \\
\hline Case & $\mathrm{H} / \mathrm{U}$ & Keff & K sig & 2 2*K_sig & Keff+2^K sig \\
\hline npv001n.o & 0.5 & 0.5114 & 0.0034 & 0.0068 & 0.5182 \\
\hline npv002n.o & 1 & 0.5535 & 0.0041 & 0.0082 & 0.5617 \\
\hline npv003n.o & 2 & 0.6405 & 0.0041 & 0.0082 & 0.6487 \\
\hline npv004n.o & 3 & 0.7026 & 0.0046 & 0.0092 & 0.7118 \\
\hline npv005n.o & 3.99 & 0.7556 & 0.0049 & 0.0098 & 0.7654 \\
\hline npv006n.o & 4 & 0.7132 & 0.0045 & 0.009 & 0.7222 \\
\hline npv007n.o & 5 & 0.7315 & 0.0039 & 0.0078 & 0.7393 \\
\hline npv008n.o & 10 & 0.7745 & 0.0045 & 0.009 & 0.7835 \\
\hline npv009n.o & 15 & 0.7936 & 0.0052 & 0.0104 & 0.804 \\
\hline npvo10n.o & 20 & 0.7775 & 0.0046 & 0.0092 & 0.7867 \\
\hline npv011n.o & 25 & 0.7799 & 0.0049 & 0.0098 & 0.7897 \\
\hline npv012n.o & 50 & 0.7045 & 0.0045 & 0.009 & 0.7135 \\
\hline npv013n.o & 100 & 0.5641 & 0.0035 & 0.007 & 0.5711 \\
\hline npv014n.o & 150 & 0.4753 & 0.0029 & 0.0058 & 0.4811 \\
\hline npv015n.o & 200 & 0.4032 & 0.0028 & 0.0056 & 0.4088 \\
\hline
\end{tabular}


POEF-SH-30

Figure 4.4.2-4. Two 5.00" ID $\times 24$ "Tall Cylinders of UO ${ }_{2} \mathrm{~F}_{2}$ One Foot from a Wall

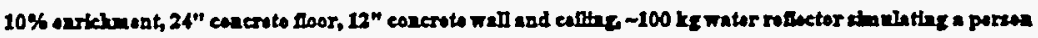

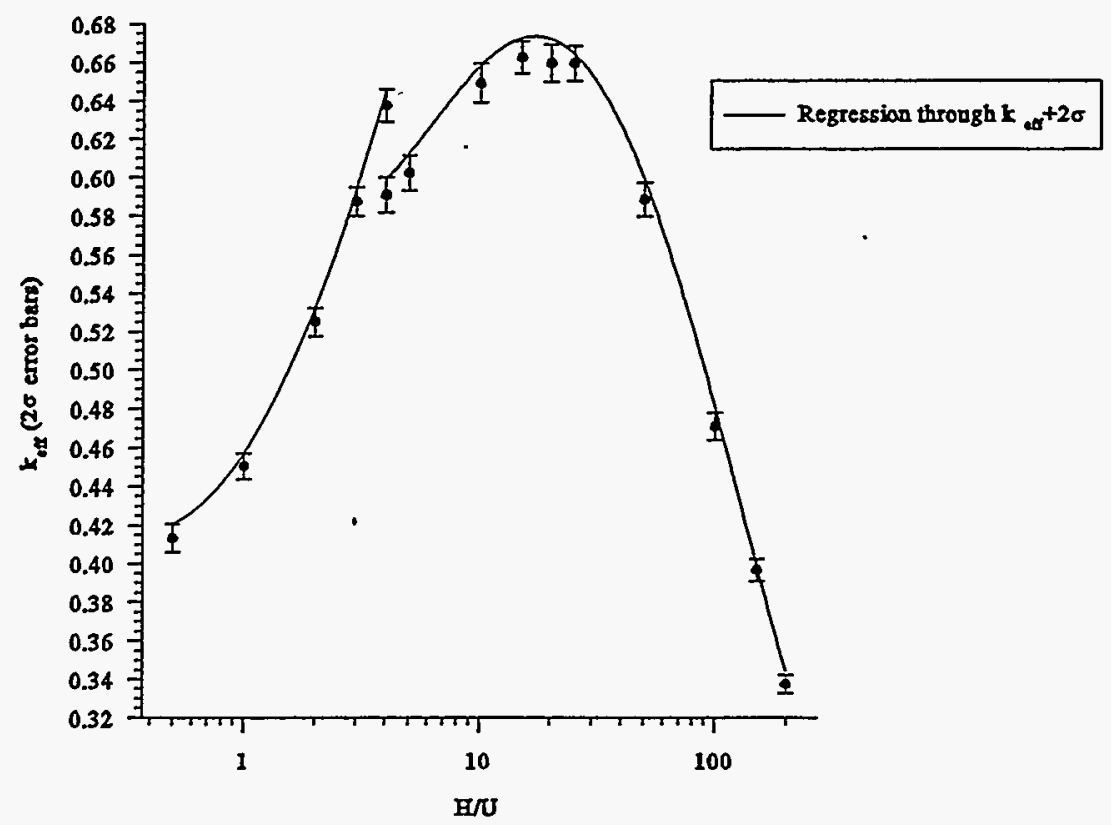

\begin{tabular}{|c|c|c|c|c|c|}
\hline \multicolumn{6}{|c|}{ Two 5.0 in. Diameter Poly. Containers 1 ' from Concrete Wall } \\
\hline \multicolumn{6}{|c|}{ for $10 \%$ Enriched UO2F2+H2O } \\
\hline Case & $\mathrm{H} / \mathrm{U}$ & Keff & K sig & $2^{\star} \mathrm{K}$ sig & Keff $+2^{*} \mathrm{~K}$ sig \\
\hline apv001n.o & 0.5 & 0.4134 & 0.0037 & 0.0074 & 0.4208 \\
\hline apv002n.o & 1 & 0.4502 & 0.0035 & 0.007 & 0.4572 \\
\hline apv003n.o & 2 & 0.5248 & 0.0037 & 0.0074 & 0.5322 \\
\hline apv004n.o & 3 & 0.587 & 0.0037 & 0.0074 & 0.5944 \\
\hline apv005n.o & 3.99 & 0.6374 & 0.0042 & 0.0084 & 0.6458 \\
\hline apv006n.o & 4 & 0.5905 & 0.0045 & 0.009 & 0.5995 \\
\hline apv007n.o & 5 & 0.602 & 0.0046 & 0.0092 & 0.6112 \\
\hline apv008n.o & 10 & 0.6489 & 0.0051 & 0.0102 & 0.6591 \\
\hline apvo09n.o & 15 & 0.6621 & 0.0042 & 0.0084 & 0.6705 \\
\hline apv010n.o & 20 & 0.659 & 0.0048 & 0.0096 & 0.6686 \\
\hline apv011n.o & 25 & 0.6588 & 0.0045 & 0.009 & 0.6678 \\
\hline apv012n.o & 50 & 0.5879 & 0.0044 & 0.0088 & 0.5967 \\
\hline apv013n.o & 100 & 0.4706 & 0.0035 & 0.007 & 0.4776 \\
\hline apv014n.o & 150 & 0.3963 & 0.0029 & 0.0058 & 0.4021 \\
\hline apv015n.o & 200 & 0.3374 & 0.0025 & 0.005 & 0.3424 \\
\hline
\end{tabular}


Figure 4.4.2-5. Single Fully Reflected 5.00" ID x 24" Tall Cylinder of $\mathrm{UF}_{4}$ and Oil $10 \%$ enrichment, $24^{n}$ concrete floor, $12^{n}$ (minimum) water reflector on all other sides

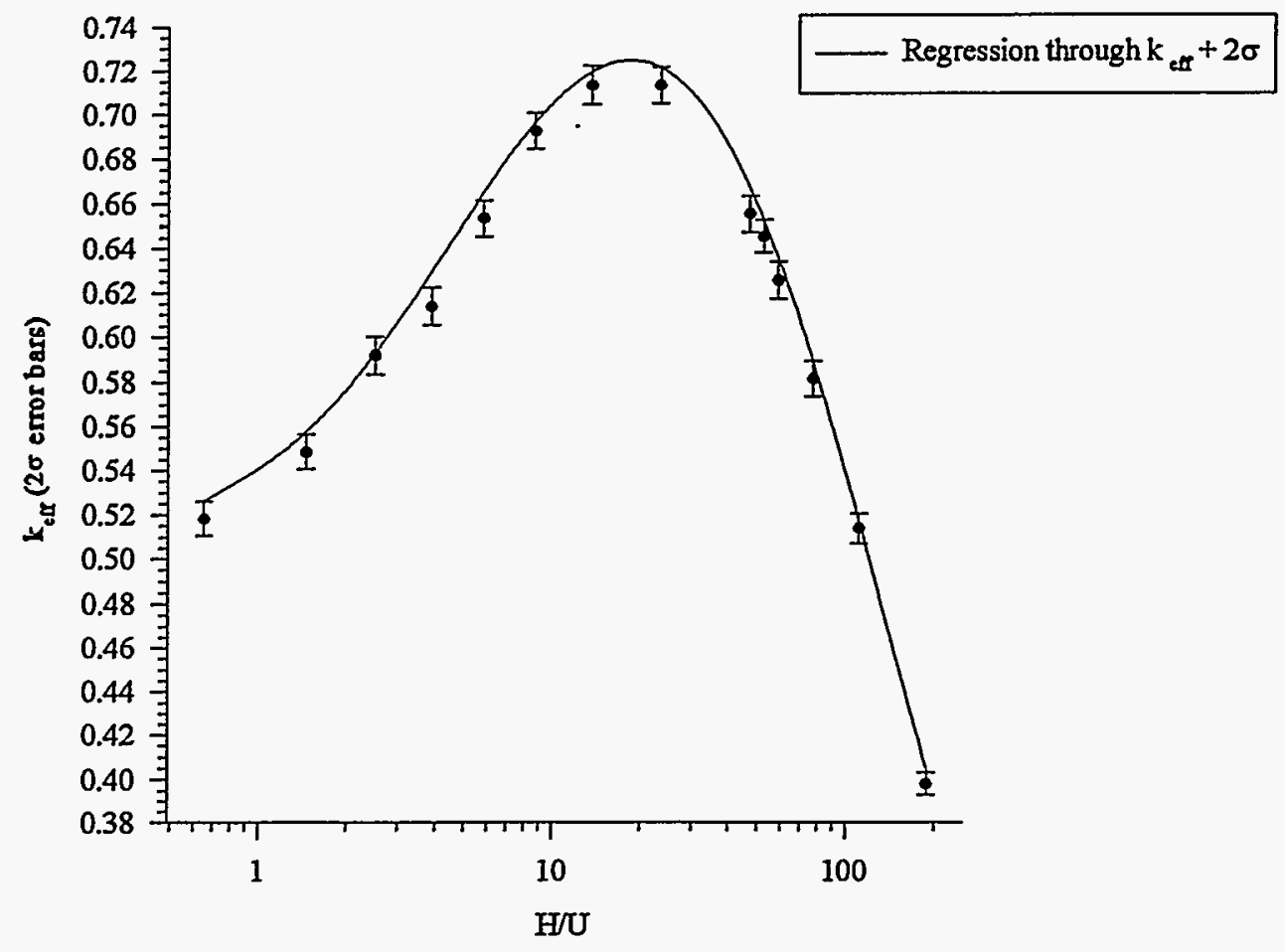

Single 5.0 in. Diameter Poly. Container w/ Full Reflection for $10 \%$ Enriched UF4+oil

\begin{tabular}{|c|c|c|c|c|c|}
\hline Case & $\mathrm{H} / \mathrm{U}$ & Keff & K_sig & 2*K_sig $^{\star *}$ & Keff+2*K_sig \\
\hline fr-500.p65.o & 0.65 & 0.5183 & 0.0038 & 0.0076 & 0.5259 \\
\hline$f r-500.145 .0$ & 1.45 & 0.5486 & 0.004 & 0.008 & 0.5566 \\
\hline$f r-500.249 .0$ & 2.49 & 0.5917 & 0.0041 & 0.0082 & 0.5999 \\
\hline fr-500.388.o & 3.88 & 0.6137 & 0.0042 & 0.0084 & 0.6221 \\
\hline fr-500.582.o & 5.82 & 0.6532 & 0.004 & 0.008 & 0.6612 \\
\hline fr-500.873.0 & 8.73 & 0.6922 & 0.004 & 0.008 & 0.7002 \\
\hline fr-500.136.o & 13.6 & 0.713 & 0.0045 & 0.009 & 0.722 \\
\hline$f r-500.233 .0$ & 23.3 & 0.7128 & 0.0041 & 0.0082 & 0.721 \\
\hline$f r-500.471 .0$ & 47.1 & 0.6551 & 0.004 & 0.008 & 0.6631 \\
\hline $\mathrm{fr}-500.524 .0$ & 52.4 & 0.6451 & 0.0036 & 0.0072 & 0.6523 \\
\hline fr-500.588.o & 58.8 & 0.6252 & 0.0041 & 0.0082 & 0.6334 \\
\hline fr-500.773.o & 77.3 & 0.5811 & 0.0039 & 0.0078 & 0.5889 \\
\hline fr-500.110.0 & 110.6 & 0.5138 & 0.0034 & 0.0068 & 0.5206 \\
\hline fr-500.188.0 & 188.2 & 0.3981 & 0.0026 & 0.0052 & 0.4033 \\
\hline
\end{tabular}


Figure 4.4.2-6. Single 5.00" ID x 24" Tall Cylinder of $\mathrm{UF}_{4}$ and Oil in a Concrete Corner $10 \%$ enrichment, 24 " concrete floor, 12 " concrete walls and ceiling, $\sim 100 \mathrm{~kg}$ water reflector simulating a person

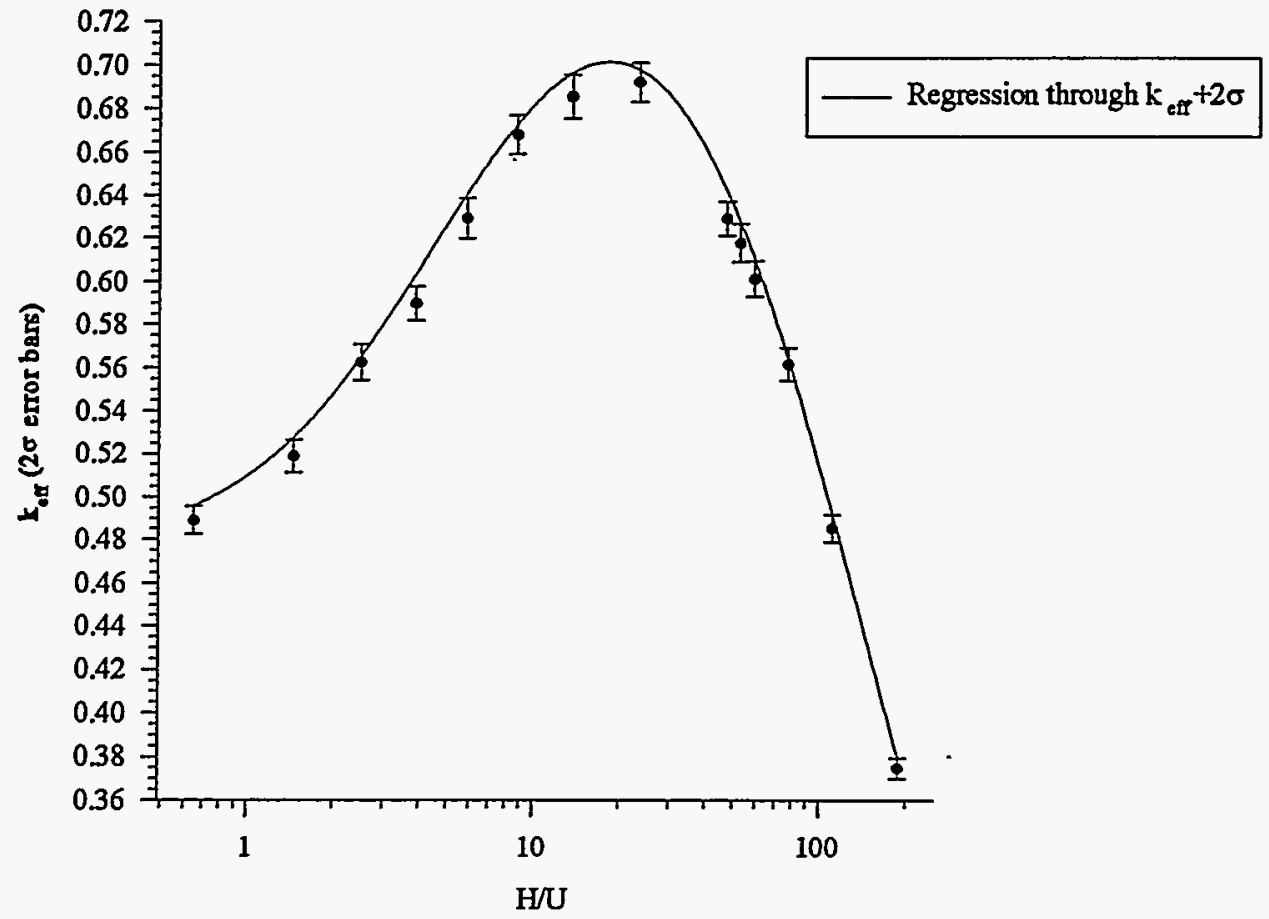

\begin{tabular}{|c|c|c|c|c|c|}
\hline \multicolumn{6}{|c|}{ Single 5.0 in. Diameter Poly. Container in a Corner } \\
\hline \multicolumn{6}{|c|}{ for $10 \%$ Enriched UF4+oil } \\
\hline Case & $\mathrm{H} / \mathrm{U}$ & Keff & K_sig & $2^{\star} \mathrm{K}$ sig & Keff $+2^{*} \mathrm{~K}$ sig \\
\hline cr-500.p65.0 & 0.65 & 0.489 & 0.0033 & 0.0066 & 0.4956 \\
\hline cr-500.145.0 & 1.45 & 0.5188 & 0.0038 & 0.0076 & 0.5264 \\
\hline cr-500.249.0 & 2.49 & 0.5625 & 0.0042 & 0.0084 & 0.5709 \\
\hline cr-500.388.0 & 3.88 & 0.5895 & 0.004 & 0.008 & 0.5975 \\
\hline cr-500.582.0 & 5.82 & 0.629 & 0.0047 & 0.0094 & 0.6384 \\
\hline cr-500.873.0 & 8.73 & 0.6676 & 0.0045 & 0.009 & 0.6766 \\
\hline cr-500.136.o & 13.6 & 0.6849 & 0.005 & 0.01 & 0.6949 \\
\hline$c r-500.233 .0$ & 23.3 & 0.6913 & 0.0045 & 0.009 & 0.7003 \\
\hline cr-500.471.0 & 47.1 & 0.6285 & 0.004 & 0.008 & 0.6365 \\
\hline cr-500.524.o & 52.4 & 0.6173 & 0.0043 & 0.0086 & 0.6259 \\
\hline cr-500.588.o & 58.8 & 0.6008 & 0.0042 & 0.0084 & 0.6092 \\
\hline $\mathrm{cr}-500.773 .0$ & 77.3 & 0.5612 & 0.0038 & 0.0076 & 0.5688 \\
\hline cr-500.110.0 & 110.6 & 0.485 & 0.0032 & 0.0064 & 0.4914 \\
\hline cr-500.188.0 & 188.2 & 0.3747 & 0.0024 & 0.0048 & 0.3795 \\
\hline
\end{tabular}


Figure 4.4.2-7. Two 5.00" ID $\times$ 24" Tall Cylinders of UF and Oil Next to a Wall $10 \%$ enfichment, $24^{\prime \prime}$ concreto floor, 12" concrote wall and colling, -100 kg wator reflector sionalatiog a person

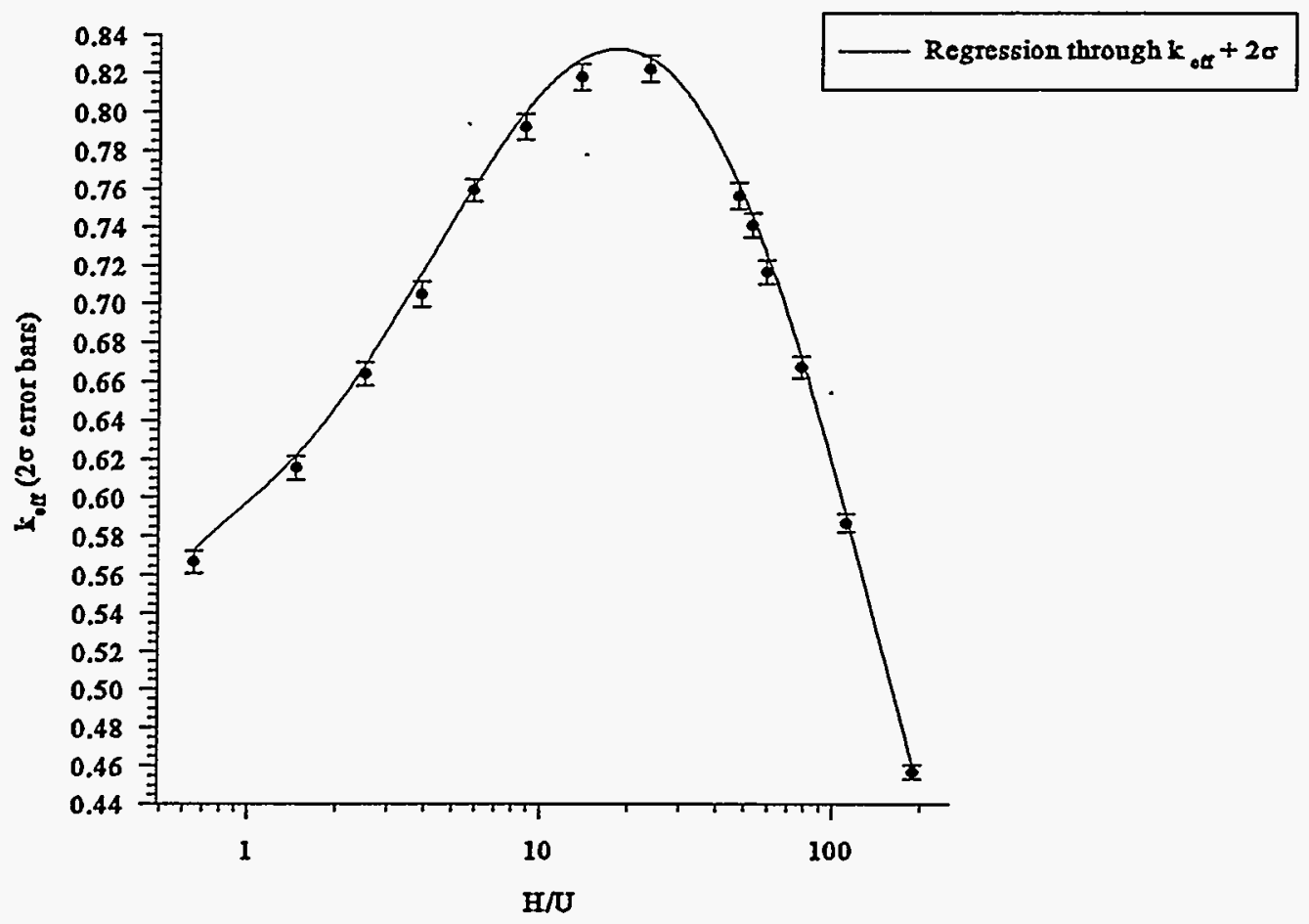

Two 5.0 in. Diameter Poly. Containers Adjacent to Concrete Wall for $10 \%$ Enriched UF4+oil

\begin{tabular}{|c|c|c|c|c|c|}
\hline \hline Case & H/U & Keff & K sig & $2^{\star} K$ sig & Keff+2*K_sig \\
\hline d00n-500.p65 & 0.65 & 0.5669 & 0.00291 & 0.00582 & 0.57272 \\
\hline$d 00 n-500.145$ & 1.45 & 0.6155 & 0.00308 & 0.00616 & 0.62166 \\
\hline$d 00 n-500.249$ & 2.49 & 0.6637 & 0.00295 & 0.0059 & 0.6696 \\
\hline$d 00 n-500.388$ & 3.88 & 0.7043 & 0.00332 & 0.00664 & 0.71094 \\
\hline$d 00 n-500.582$ & 5.82 & 0.7587 & 0.00304 & 0.00608 & 0.76478 \\
\hline$d 00 n-500.873$ & 8.73 & 0.7915 & 0.0033 & 0.0066 & 0.7981 \\
\hline$d 00 n-500.136$ & 13.6 & 0.817 & 0.0035 & 0.007 & 0.824 \\
\hline$d 00 n-500.233$ & 23.3 & 0.8211 & 0.00342 & 0.00684 & 0.82794 \\
\hline$d 00 n-500.471$ & 47.1 & 0.7555 & 0.00351 & 0.00702 & 0.76252 \\
\hline$d 00 n-500.524$ & 52.4 & 0.74 & 0.00304 & 0.00608 & 0.74608 \\
\hline$d 00 n-500.588$ & 58.8 & 0.7158 & 0.00321 & 0.00642 & 0.72222 \\
\hline$d 00 n-500.773$ & 77.3 & 0.6666 & 0.00267 & 0.00534 & 0.67194 \\
\hline$d 00 n-500.110$ & 110.6 & 0.5864 & 0.00232 & 0.00464 & 0.59104 \\
\hline$d 00 n-500.188$ & 188.2 & 0.4569 & 0.00179 & 0.00358 & 0.46048 \\
\hline
\end{tabular}




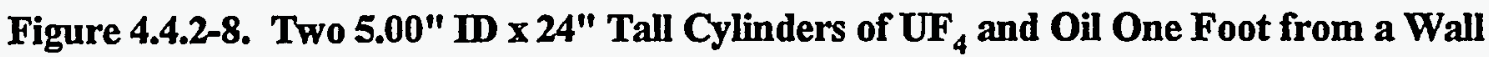
$10 \%$ enrichment, 24 " concrete floor, 12 " concrete wall and ceiling, $\sim 100 \mathrm{~kg}$ water reflector simulating a person

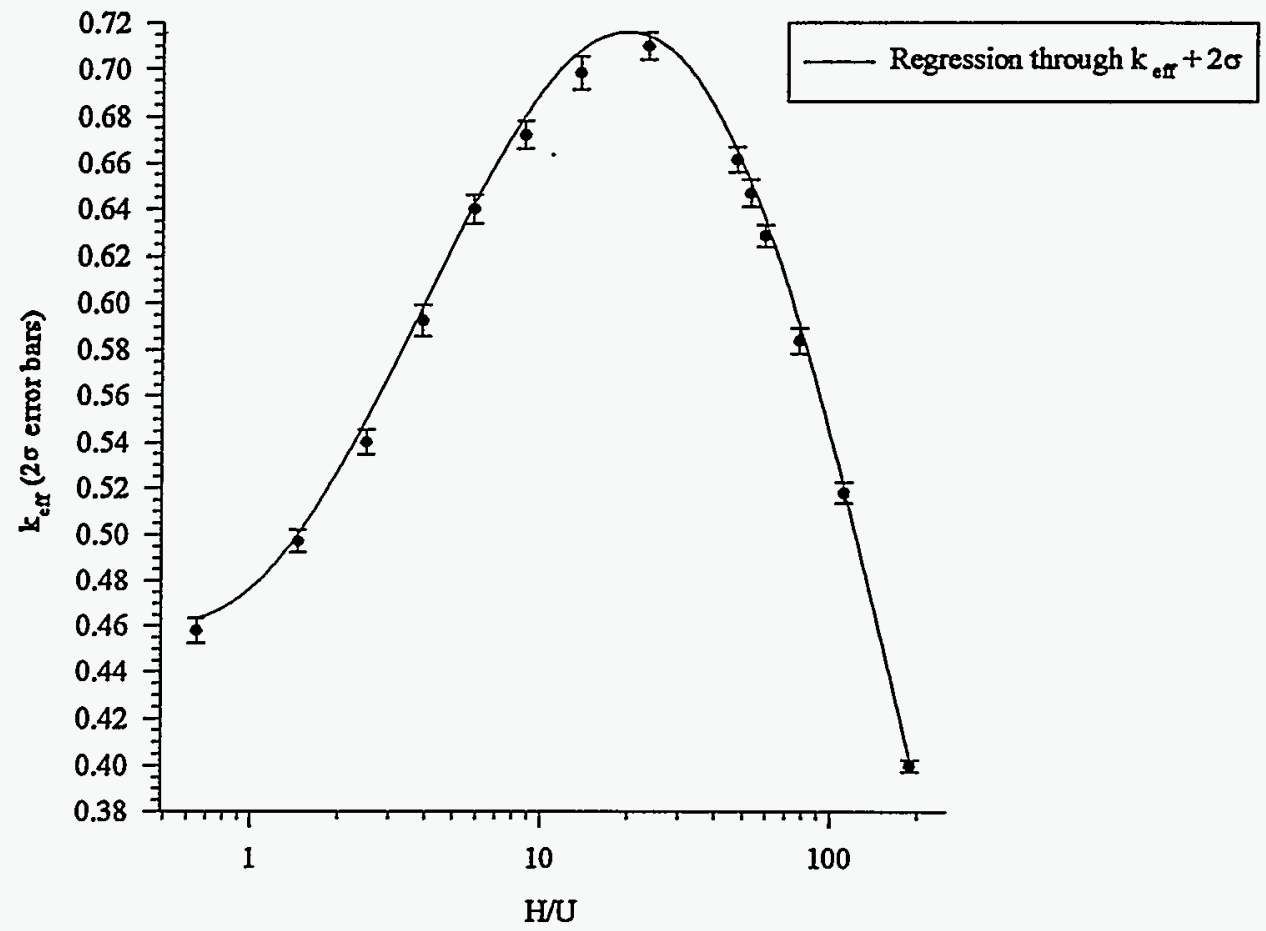

Two 5.0 in. Diameter Poly. Containers 1' from Concrete Wall for $10 \%$ Enriched UF4+oil

\begin{tabular}{|c|c|c|c|c|c|}
\hline \hline Case & H/U & Keff & K_sig & $2^{\star}$ K sig & Keff+2* K sig \\
\hline \hline$d 12 n-500 . p 65$ & 0.65 & 0.4579 & 0.00265 & 0.0053 & 0.4632 \\
\hline$d 12 n-500.145$ & 1.45 & 0.4972 & 0.00245 & 0.0049 & 0.5021 \\
\hline$d 12 n-500.249$ & 2.49 & 0.5399 & 0.00275 & 0.0055 & 0.5454 \\
\hline$d 12 n-500.388$ & 3.88 & 0.5924 & 0.00336 & 0.00672 & 0.59912 \\
\hline$d 12 n-500.582$ & 5.82 & 0.6396 & 0.00303 & 0.00606 & 0.64566 \\
\hline$d 12 n-500.873$ & 8.73 & 0.6718 & 0.00299 & 0.00598 & 0.67778 \\
\hline$d 12 n-500.136$ & 13.6 & 0.6979 & 0.00355 & 0.0071 & 0.705 \\
\hline$d 12 n-500.233$ & 23.3 & 0.7092 & 0.00299 & 0.00598 & 0.71518 \\
\hline$d 12 n-500.471$ & 47.1 & 0.661 & 0.00273 & 0.00546 & 0.66646 \\
\hline$d 12 n-500.524$ & 52.4 & 0.6464 & 0.00303 & 0.00606 & 0.65246 \\
\hline$d 12 n-500.588$ & 58.8 & 0.628 & 0.00242 & 0.00484 & 0.63284 \\
\hline$d 12 n-500.773$ & 77.3 & 0.5834 & 0.00272 & 0.00544 & 0.58884 \\
\hline$d 12 n-500.110$ & 110.6 & 0.5177 & 0.00217 & 0.00434 & 0.52204 \\
\hline$d 12 n-500.188$ & 188.2 & 0.3999 & 0.001184 & 0.002368 & 0.402268 \\
\hline
\end{tabular}


POEF-SH-30

\subsubsection{0-inch-Diameter Case ( $\left(\mathrm{UF}_{4}+\mathrm{Oil}\right)$}

The $\mathrm{UF}_{4}+$ oil is more reactive than $\mathrm{UO}_{2} \mathrm{~F}_{2}$. To approve the 5.0-inch container for use with $\mathrm{UO}_{2} \mathrm{~F}_{2}$ only, the container must be shown to be safe when filled with the more reactive material ( $\left.\mathrm{UF}_{4}+\mathrm{oil}\right)$. The model consists of a single container reflected by 1 inch of close-fitting water on the top and sides and 24 inches of concrete on the bottom. The data for these calculations are presented in Table and Figure 4.4.3-1. The calculated results fall within the safety criterion.

\subsection{25-inch-Diameter by 24-inch-Tall Tin-Plated Steel Container}

This section presents results of calculations designed to model the F-Can and Z-Can containers described in Table 1.0-1.

\subsection{1 $100 \%$ Enriched $\mathrm{UO}_{2} \mathrm{~F}_{2}+\mathrm{H}_{2} \mathrm{O}$ and $\mathrm{UF}_{4}+\mathrm{Oil}$}

Limiting the uranium density for $\mathrm{H} / \mathrm{U}$ values less than 4.0 was not done for this container type, because a second $k_{\text {eff }}$ peak greater than the approval criterion occurred at $\mathrm{H} / \mathrm{U}$ values greater than 4.0. Table 4.5.1-0 gives the figure and table numbers that contain the result data for this container with various geometry

- models and fuel materials. The geometry model designators are explained in Table 4.1-1.

The standard arrays were not analyzed because the single container and two container case results fell outside the safety criterion. Container thickness was not included in the models tested because single container full reflection results fell outside the safety criterion for both $\mathrm{UO}_{2} \mathrm{~F}_{2}+\mathrm{H}_{2} \mathrm{O}$ and $\mathrm{UF}_{4}+$ oil. 
Figure 4.4.3-1. Single 5.00" ID $\times 24$ " Tall Cylinder of $\mathrm{UF}_{4}$ and Oil $100 \%$ enrichment, $195^{\prime \prime}$ container, $1^{\prime \prime}$ close fit water reflector on all other sides, $12^{\prime \prime}$ ceiling $10^{\prime}$ above

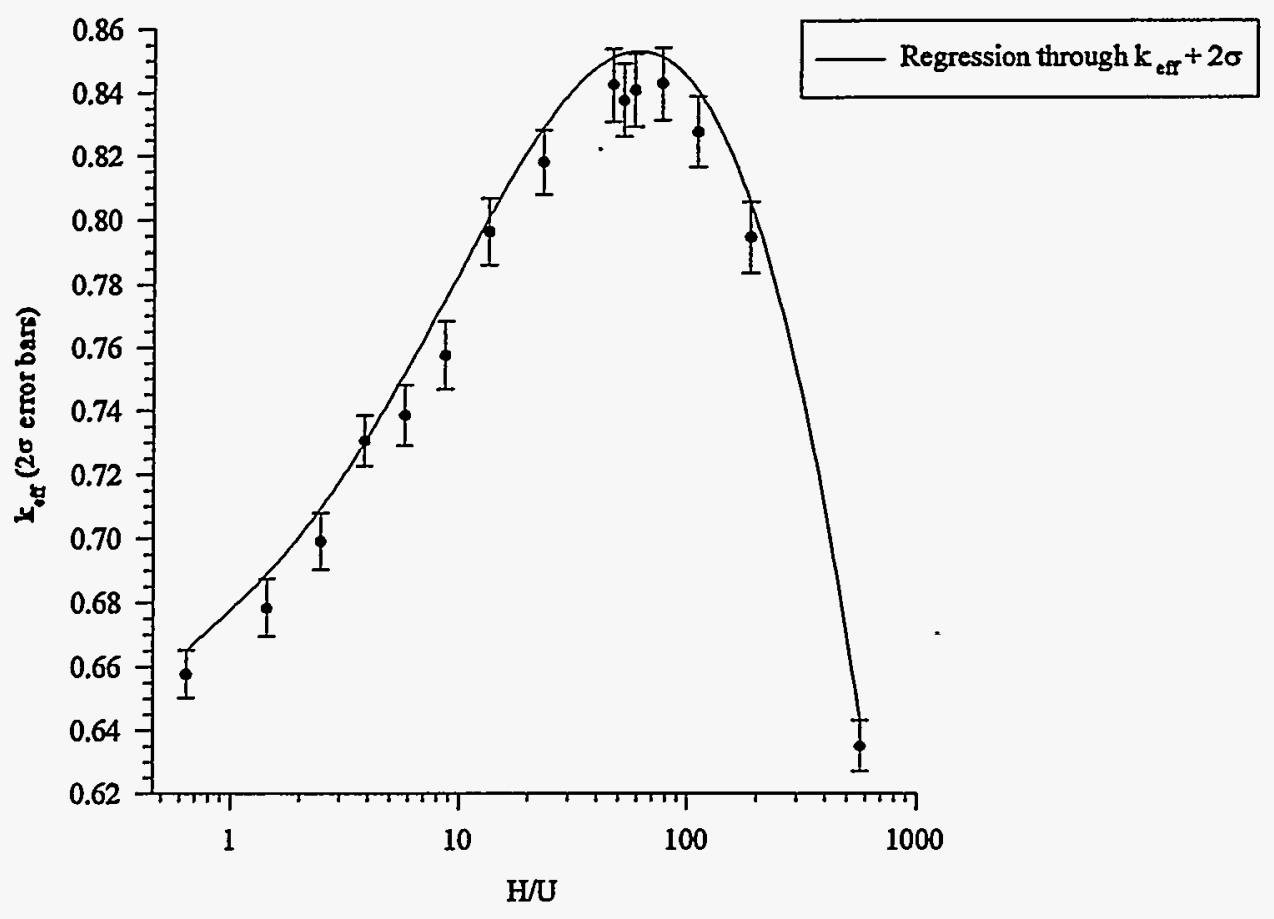

\begin{tabular}{|c|c|c|c|c|c|}
\hline Single 5. & 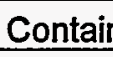 & $n$ & tect & $1 \mathrm{Co}$ & \\
\hline For $100 \%$ & hed UI & & & & \\
\hline Case & $\mathrm{H} / \mathrm{U}$ & Keff & K_sig & $2^{\star} \mathrm{K}$ _sig & Keff+2*K_sig \\
\hline cye01n-c & 0.64 & 0.6578 & 0.0038 & 0.0076 & 0.6654 \\
\hline cye02n-c & 1.44 & 0.6784 & 0.0045 & 0.009 & 0.6874 \\
\hline cye03n-c & 2.47 & 0.6992 & 0.0044 & 0.0088 & 0.708 \\
\hline cyeo4n-c & 3.84 & 0.7306 & 0.004 & 0.008 & 0.7386 \\
\hline cye05n-c & 5.76 & 0.7385 & 0.0047 & 0.0094 & 0.7479 \\
\hline cye06n-c & 8.64 & 0.7575 & 0.0054 & 0.0108 & 0.7683 \\
\hline cye07n-c & 13.4 & 0.7963 & 0.0051 & 0.0102 & 0.8065 \\
\hline cye08n-c & 23.1 & 0.8178 & 0.005 & 0.01 & 0.8278 \\
\hline cye09n-c & 46.6 & 0.8421 & 0.0057 & 0.0114 & 0.8535 \\
\hline cye10n-c & 51.9 & 0.8373 & 0.0057 & 0.0114 & 0.8487 \\
\hline cye11n-c & 58.2 & 0.8404 & 0.0057 & 0.0114 & 0.8518 \\
\hline cye12n-c & 76.6 & 0.8424 & 0.0057 & 0.0114 & 0.8538 \\
\hline cye13n-c & 109.5 & 0.8272 & 0.0056 & 0.0112 & 0.8384 \\
\hline cye14n-c & 186.4 & 0.7944 & 0.0054 & 0.0108 & 0.8052 \\
\hline cye15n-c & 570.6 & 0.6351 & 0.004 & 0.008 & 0.6431 \\
\hline
\end{tabular}


POEF-SH-30

Table 4.5.1-0. Summary of References for F-Can Results for $100 \%$ Enrichment

\begin{tabular}{|c|c|c|}
\hline Geometry Model & Material & Figure/Table Number \\
\hline \hline SFR & $\mathrm{UO}_{2} \mathrm{~F}_{2}+\mathrm{H}_{2} \mathrm{O}$ & $4.5 .1-1$ \\
\hline $\mathrm{SCC}$ & $\mathrm{UO}_{2} \mathrm{~F}_{2}+\mathrm{H}_{2} \mathrm{O}$ & $4.5 .1-2$ \\
\hline $\mathrm{TACW}$ & $\mathrm{UO}_{2} \mathrm{~F}_{2}+\mathrm{H}_{2} \mathrm{O}$ & $4.5 .1-3$ \\
\hline $\mathrm{TSCW}$ & $\mathrm{UO}_{2} \mathrm{~F}_{2}+\mathrm{H}_{2} \mathrm{O}$ & $4.5 .1-4$ \\
\hline $\mathrm{SFR}$ & $\mathrm{UF}_{4}+\mathrm{oil}$ & $4.5 .1-5$ \\
\hline $\mathrm{SCC}$ & $\mathrm{UF}_{4}+$ oil & $4.5 .1-6$ \\
\hline $\mathrm{TACW}$ & $\mathrm{UF}_{4}+$ oil & $4.5 .1-7$ \\
\hline TSCW & $\mathrm{UF}_{4}+$ oil & $4.5 .1-8$ \\
\hline SMRC & $\mathrm{UF}_{4}+$ oil & $4.5 .1-9$ \\
\hline
\end{tabular}


Figure 4.5.1-1. Single Fully Reflected 5.25" ID $\times 24$ " Tall Cylinder of $\mathrm{UO}_{2} \mathrm{~F}_{2}$ $100 \%$ enrichment, 24 " concrete floor, 12 " (minimum) water reflector on all other sides

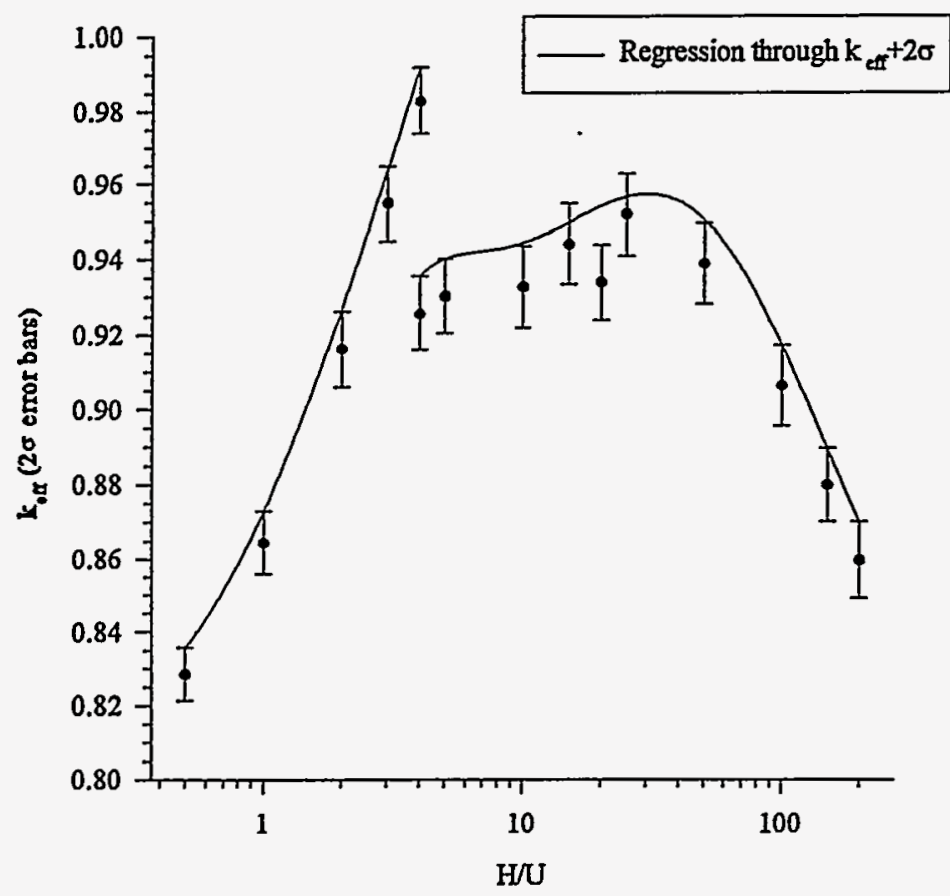

\begin{tabular}{|c|c|c|c|c|c|}
\hline \multicolumn{6}{|c|}{ for $100 \%$ Enriched UO2F $2+\mathrm{H} 2 \mathrm{O}$} \\
\hline Case & $\mathrm{H} / \mathrm{U}$ & Keff & K_sig & 2*K_sig. & Keff $+2^{*} K$ sig \\
\hline frcoo1n.o & 0.5 & 0.8285 & 0.0036 & 0.0072 & 0.8357 \\
\hline frc002n.o & 1 & 0.8645 & 0.0043 & 0.0086 & 0.8731 \\
\hline frc003n.o & 2 & 0.9161 & 0.0051 & 0.0102 & 0.9263 \\
\hline frco04n.o & 3 & 0.9548 & 0.005 & 0.01 & 0.9648 \\
\hline frco05n.o & 3.99 & 0.9828 & 0.0045 & 0.009 & 0.9918 \\
\hline frc006n.o & 4 & 0.9256 & 0.0049 & 0.0098 & 0.9354 \\
\hline frc007n.o & 5 & 0.9302 & 0.0049 & 0.0098 & 0.94 \\
\hline frc008n.o & 10 & 0.9326 & 0.0054 & 0.0108 & 0.9434 \\
\hline frco09n.o & 15 & 0.9439 & 0.0053 & 0.0106 & 0.9545 \\
\hline frco10n.o & 20 & 0.9337 & 0.005 & 0.01 & 0.9437 \\
\hline frco11n.o & 25 & 0.9516 & 0.0055 & 0.011 & 0.9626 \\
\hline frco12n.o & 50 & 0.9387 & 0.0053 & 0.0106 & 0.9493 \\
\hline frc013n.o & 100 & 0.9062 & 0.0054 & 0.0108 & 0.917 \\
\hline frco14n.o & 150 & 0.8798 & 0.0048 & 0.0096 & 0.8894 \\
\hline frc015n.o & 200 & 0.8596 & 0.0052 & 0.0104 & 0.87 \\
\hline
\end{tabular}


Figure 4.5.1-2. Single 5.25" $\mathrm{D}$ × 24" Tall Cylinder of $\mathrm{UO}{ }_{2} \mathrm{~F}_{2}$ in a Concrete Corner

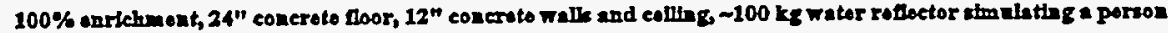

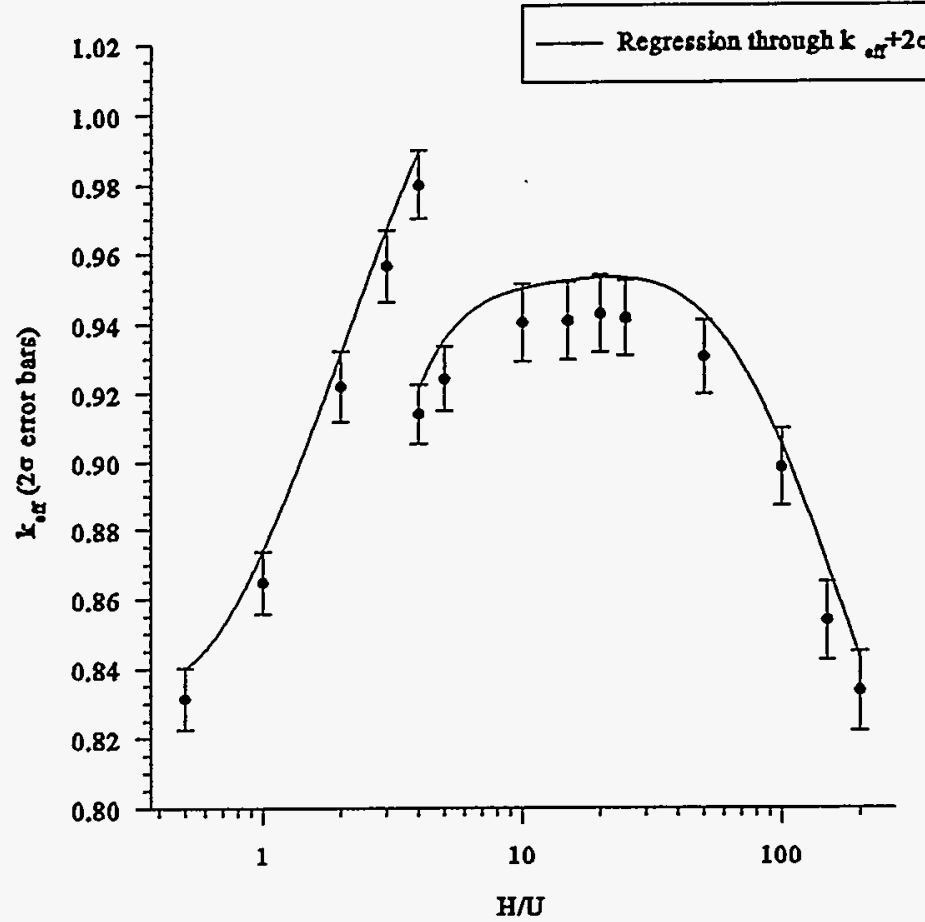

Single 5.25 in. Diameter Tin Plated Steel Container in a Corner for $100 \%$ Enriched UO2F2+ $\mathrm{H} 2 \mathrm{O}$

\begin{tabular}{|c|c|c|c|c|c|}
\hline Case & H/U & Keff & K_sig & $2^{\star}$ K_sig & Keff+2* K sig \\
\hline \hline cpc001n.o & 0.5 & 0.8314 & 0.0044 & 0.0088 & 0.8402 \\
\hline cpc002n.o & 1 & 0.8647 & 0.0045 & 0.009 & 0.8737 \\
\hline cpc003n.o & 2 & 0.9218 & 0.0051 & 0.0102 & 0.932 \\
\hline cpc004n.o & 3 & 0.9563 & 0.0051 & 0.0102 & 0.9665 \\
\hline cpc005n.o & 3.99 & 0.9799 & 0.005 & 0.01 & 0.9899 \\
\hline cpc006n.o & 4 & 0.9138 & 0.0043 & 0.0086 & 0.9224 \\
\hline cpc007n.o & 5 & 0.9239 & 0.0046 & 0.0092 & 0.9331 \\
\hline cpc008n.o & 10 & 0.94 & 0.0056 & 0.0112 & 0.9512 \\
\hline cpc009n.o & 15 & 0.9405 & 0.0056 & 0.0112 & 0.9517 \\
\hline cpc010n.o & 20 & 0.9425 & 0.0055 & 0.011 & 0.9535 \\
\hline cpc011n.o & 25 & 0.9413 & 0.0054 & 0.0108 & 0.9521 \\
\hline cpc012n.o & 50 & 0.9301 & 0.0053 & 0.0106 & 0.9407 \\
\hline cpc013n.o & 100 & 0.8982 & 0.0056 & 0.0112 & 0.9094 \\
\hline cpc014n.o & 150 & 0.8537 & 0.0056 & 0.0112 & 0.8649 \\
\hline cpc015n.o & 200 & 0.8335 & 0.0057 & 0.0114 & 0.8449 \\
\hline
\end{tabular}


Figure 4.5.1-3. Two 5.25" $\mathrm{D} \times 24$ " Tall Cylinders of $\mathrm{UO}_{2} \mathrm{~F}_{2}$ Next to a Wall $100 \%$ enrlchment, $24^{\prime \prime}$ concrete floor, 12 " concrete wall and ceiling, $-100 \mathrm{~kg}$ water reflector simulating a person

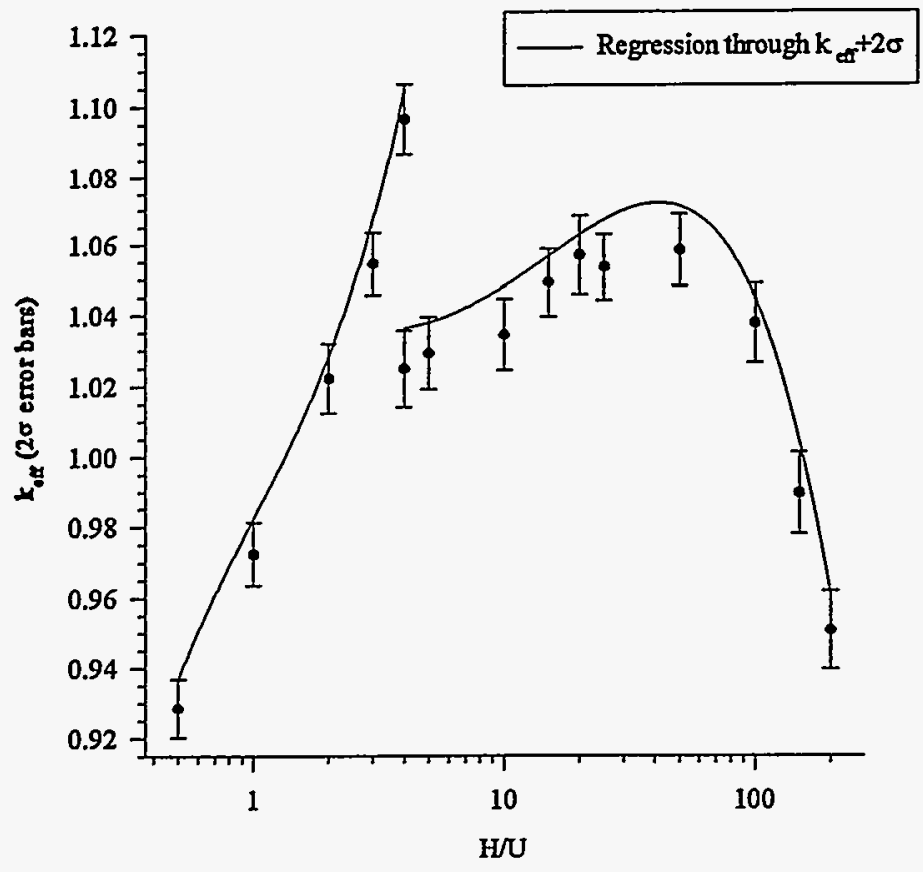

\begin{tabular}{|c|c|c|c|c|c|}
\hline \multicolumn{6}{|c|}{ Two 5.25 in. Dia. Tin/Steel Containers Adjacent to Concrete Wall } \\
\hline \multicolumn{6}{|c|}{ for $100 \%$ Enriched UO2F2+H2O } \\
\hline Case & HN & Keff & K_sig & $2^{*} \mathrm{~K}$ sig & Keff $+2^{*} K$ sig \\
\hline npc001n.o & 0.5 & 0.9286 & 0.0042 & 0.0084 & 0.937 \\
\hline npc002n.o & 1 & 0.9725 & 0.0044 & 0.0088 & 0.9813 \\
\hline npc003n.o & 2 & 1.0222 & 0.0049 & 0.0098 & 1.032 \\
\hline npc004n.o & 3 & 1.0545 & 0.0045 & 0.009 & 1.0635 \\
\hline npc005n.o & 3.99 & 1.0964 & 0.0049 & 0.0098 & 1.1062 \\
\hline npc006n.o & 4 & 1.0248 & 0.0054 & 0.0108 & 1.0356 \\
\hline npc007n.o & 5 & 1.0291 & 0.0051 & 0.0102 & 1.0393 \\
\hline npc008n.o & 10 & 1.0342 & 0.005 & 0.01 & 1.0442 \\
\hline npc009n.o & 15 & 1.049 & 0.0048 & 0.0096 & 1.0586 \\
\hline npc010n.o & 20 & 1.0569 & 0.0057 & 0.0114 & 1.0683 \\
\hline npc011n.o & 25 & 1.0533 & 0.0047 & 0.0094 & 1.0627 \\
\hline npc012n.o & 50 & 1.0582 & 0.0052 & 0.0104 & 1.0686 \\
\hline npc013n.o & 100 & 1.0375 & 0.0056 & 0.0112 & 1.0487 \\
\hline npc014n.o & 150 & 0.9895 & 0.0058 & 0.0116 & 1.0011 \\
\hline npc015n.o & 200 & 0.9507 & 0.0056 & 0.0112 & 0.9619 \\
\hline
\end{tabular}


Figure 4.5.1-4. Two 5.25" $\mathrm{ID} \times 24$ " Tall Cylinders of $\mathrm{UO}_{2} \mathrm{~F}_{2}$ One Foot from a Wall $100 \%$ enrichment, 24 " concrete floor, 12" concrete wall and ceiling, $100 \mathrm{~kg}$ water reflector simulating a person

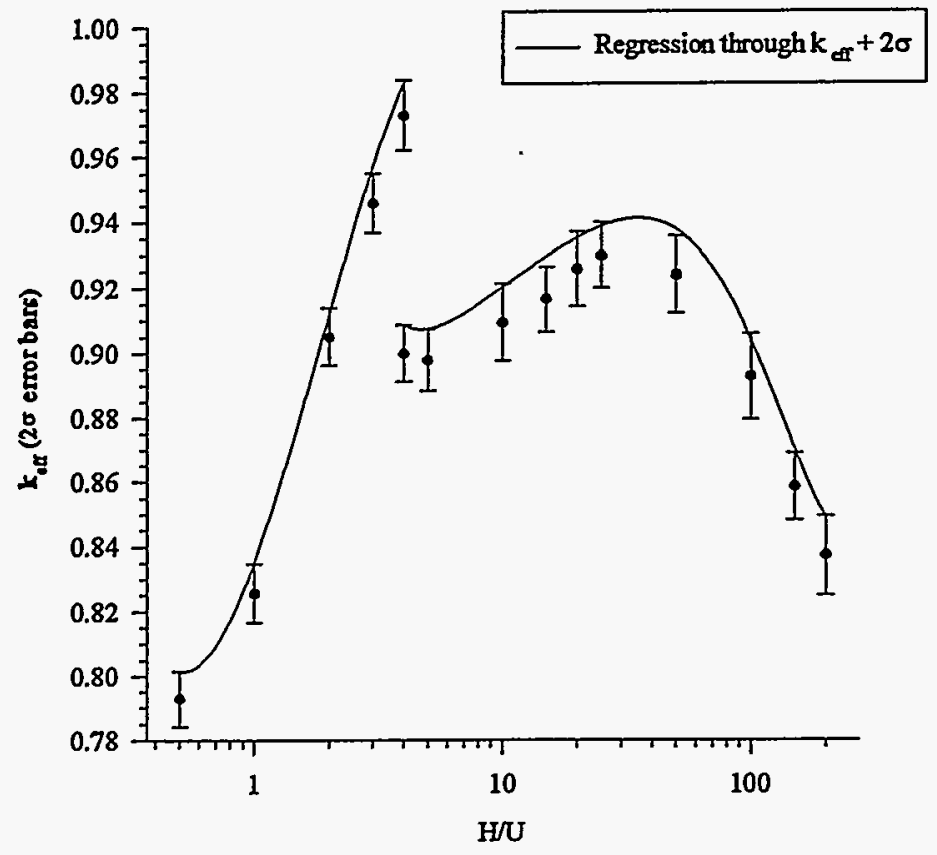

\begin{tabular}{|c|c|c|c|c|c|}
\hline \multicolumn{6}{|c|}{ for $100 \%$ Enriched UO2F2+H2O } \\
\hline Case & $H / U$ & Keff & K_sig & $2^{\star} \mathrm{K}$ sig & Keff $+2^{\star} K$ sig \\
\hline apc001n.o & 0.5 & 0.7929 & 0.0043 & 0.0086 & 0.8015 \\
\hline apc002n.o & 1 & 0.8256 & 0.0045 & 0.009 & 0.8346 \\
\hline apc003n.o & 2 & 0.9049 & 0.0044 & 0.0088 & 0.9137 \\
\hline apc004n.o & 3 & 0.9456 & 0.0046 & 0.0092 & 0.9548 \\
\hline apc005n.o & 3.99 & 0.9728 & 0.0054 & 0.0108 & 0.9836 \\
\hline apc006n.o & 4 & 0.8997 & 0.0044 & 0.0088 & 0.9085 \\
\hline apc007n.o & 5 & 0.8976 & 0.0048 & 0.0096 & 0.9072 \\
\hline apc008n.o & 10 & 0.9091 & 0.0059 & 0.0118 & 0.9209 \\
\hline apc009n.o & 15 & 0.9162 & 0.005 & 0.01 & 0.9262 \\
\hline apc010n.o & 20 & 0.9255 & 0.0057 & 0.0114 & 0.9369 \\
\hline apc011n.o & 25 & 0.9296 & 0.005 & 0.01 & 0.9396 \\
\hline apc012n.o & 50 & 0.9237 & 0.0059 & 0.0118 & 0.9355 \\
\hline apc013n.o & 100 & 0.8925 & 0.0066 & 0.0132 & 0.9057 \\
\hline apc014n.o & 150 & 0.8583 & 0.0052 & 0.0104 & 0.8687 \\
\hline apc015n.o & 200 & 0.8371 & 0.0061 & 0.0122 & 0.8493 \\
\hline
\end{tabular}


Figure 4.5.1-5. Single Fully Reflected 5.25" ID $\times 24$ " Tall Cylinder of UF , and Oil 100\% anrichnent, 24" eoncroto floor, 12" (nhinwa) water reglotor en all othor abdo:

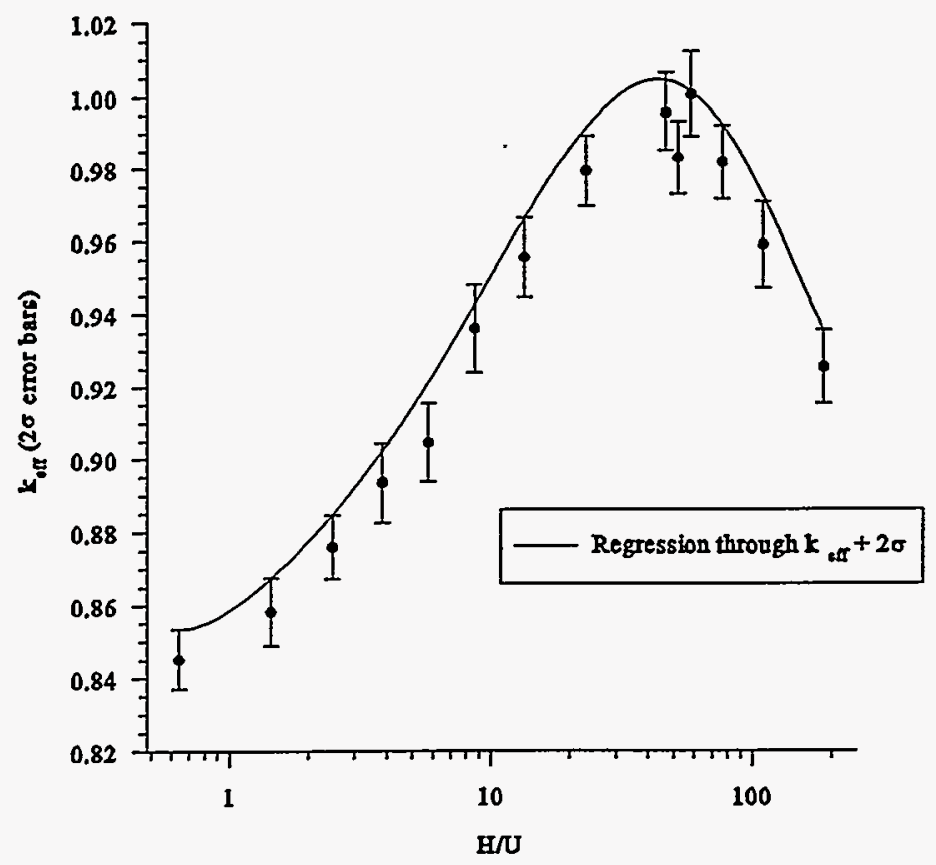

Single 5.25 in. Diameter Tin/Steel Container w/ Full Reflection for $100 \%$ Enriched UF4+oil

\begin{tabular}{|c|c|c|c|c|c|}
\hline & & & & \\
\hline Case & $H / U$ & Keff & K sig & $2^{*}$ K sig & Keff+2*K sig \\
\hline & & & & & \\
\hline frf001n.o & 0.64 & 0.8452 & 0.0041 & 0.0082 & 0.8534 \\
\hline frf002n.o & 1.44 & 0.8582 & 0.0047 & 0.0094 & 0.8676 \\
\hline frf003n.o & 2.47 & 0.8759 & 0.0043 & 0.0086 & 0.8845 \\
\hline frf004n.o & 3.84 & 0.8934 & 0.0054 & 0.0108 & 0.9042 \\
\hline frf005n.o & 5.76 & 0.9044 & 0.0054 & 0.0108 & 0.9152 \\
\hline frf006n.o & 8.64 & 0.9358 & 0.006 & 0.012 & 0.9478 \\
\hline frf007n.o & 13.4 & 0.9551 & 0.0054 & 0.0108 & 0.9659 \\
\hline frf008n.o & 23.1 & 0.979 & 0.0049 & 0.0098 & 0.9888 \\
\hline frf009n.o & 46.6 & 0.9952 & 0.0053 & 0.0106 & 1.0058 \\
\hline frf010n.o & 51.9 & 0.9826 & 0.0051 & 0.0102 & 0.9928 \\
\hline frf011n.o & 58.2 & 1.0001 & 0.0058 & 0.0116 & 1.0117 \\
\hline frf012n.o & 76.6 & 0.9813 & 0.0051 & 0.0102 & 0.9915 \\
\hline frf013n.o & 109.5 & 0.9585 & 0.0059 & 0.0118 & 0.9703 \\
\hline frf014n.o & 186.4 & 0.9252 & 0.0051 & 0.0102 & 0.9354 \\
\hline
\end{tabular}


Figare 4.5.1-6. Single 5.25" ID $~$ 24" Tall Cylinder of UF , and Oil in a Concrete Corner

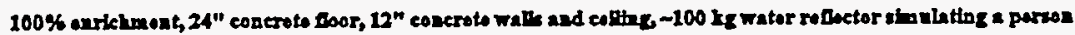

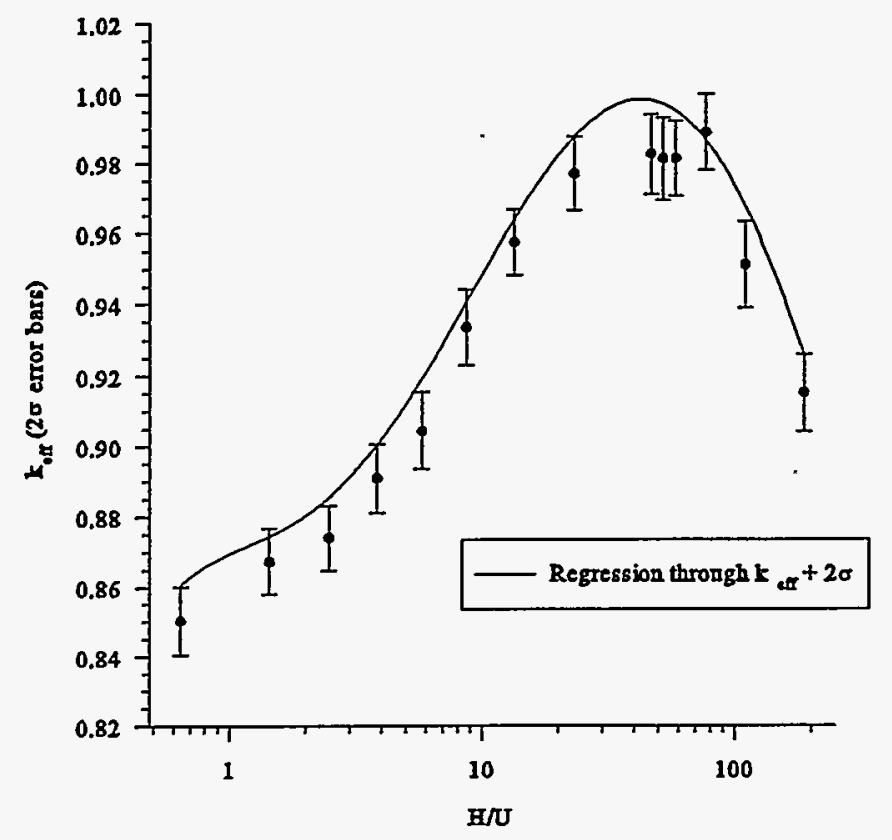

\begin{tabular}{|c|c|c|c|c|c|}
\hline \multicolumn{6}{|c|}{ Single 5.25 in. Diameter Poly. Container in a Corner } \\
\hline \multicolumn{6}{|c|}{ for $100 \%$ Enriched UF4+oil } \\
\hline Case & $H / U$ & Keff & K_sig & $2^{*} \mathrm{~K}$ sig & Keff $+2^{\star} K$ _sig \\
\hline cpe001n.o & 0.64 & 0.819 & 0.0046 & 0.0092 & 0.8282 \\
\hline cpe002n.o & 1.44 & 0.8377 & 0.0053 & 0.0106 & 0.8483 \\
\hline cpe003n.o & 2.47 & 0.8491 & 0.0049 & 0.0098 & 0.8589 \\
\hline cpe004n.o & 3.84 & 0.8667 & 0.0051 & 0.0102 & 0.8769 \\
\hline cpe005n.o & 5.76 & 0.8975 & 0.0045 & 0.009 & 0.9065 \\
\hline cpe006n.o & 8.64 & 0.8952 & 0.0045 & 0.009 & 0.9042 \\
\hline cpe007n.o & 13.4 & 0.9208 & 0.0051 & 0.0102 & 0.931 \\
\hline cpe008n.o & 23.1 & 0.9409 & 0.0051 & 0.0102 & 0.9511 \\
\hline cpe009n.o & 46.6 & 0.9583 & 0.005 & 0.01 & 0.9683 \\
\hline cpe010n.o & 51.9 & 0.9489 & 0.006 & 0.012 & 0.9609 \\
\hline cpe011n.o & 58.2 & 0.9533 & 0.0052 & 0.0104 & 0.9637 \\
\hline cpe012n.o & 76.6 & 0.9445 & 0.0057 & 0.0114 & 0.9559 \\
\hline cpe013n.o & 109.5 & 0.9292 & 0.0054 & 0.0108 & 0.94 \\
\hline cpe014n.o & 186.4 & 0.8803 & 0.006 & 0.012 & 0.8923 \\
\hline
\end{tabular}


Figure 4.5.1-7. Two 5.25" ID $~ 24$ " Tall Cylinders of UF 4 and Oil Next to a Wall $100 \%$ enrichment, 24 " concrete floor, 12" concrete wall and celling, $-100 \mathrm{~kg}$ water reflector simulating a person

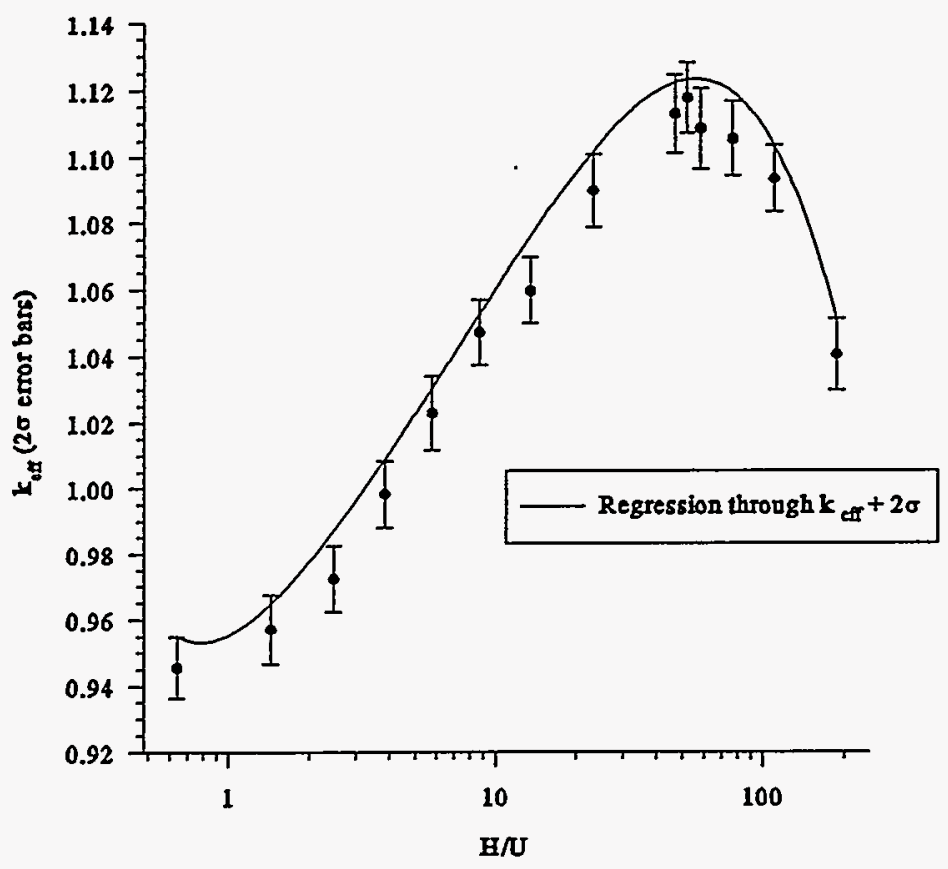

\begin{tabular}{|c|c|c|c|c|c|}
\hline \multicolumn{6}{|c|}{ Two 5.25 in. Dia. Tin/Steel Containers Adjacent to Concrete Wall } \\
\hline \multicolumn{6}{|c|}{ for $100 \%$ Enriched UF4+oil } \\
\hline Case & $H / U$ & Keff & K_sig & $2 * K$ sig & Keff $+2^{\star}$ K_sig \\
\hline npfo01n.o & 0.64 & 0.9456 & 0.0046 & 0.0092 & 0.9548 \\
\hline npf002n.o & 1.44 & 0.957 & 0.0052 & 0.0104 & 0.9674 \\
\hline npfo03n.o & 2.47 & 0.9723 & 0.005 & 0.01 & 0.9823 \\
\hline npfo04n.o & 3.84 & 0.9981 & 0.0051 & 0.0102 & 1.0083 \\
\hline npf005n.o & 5.76 & 1.0227 & 0.0056 & 0.0112 & 1.0339 \\
\hline npf006n.o & 8.64 & 1.0469 & 0.0049 & 0.0098 & 1.0567 \\
\hline npf007n.o & 13.4 & 1.0593 & 0.0049 & 0.0098 & 1.0691 \\
\hline npf008n.o & 23.1 & 1.0894 & 0.0056 & 0.0112 & 1.1006 \\
\hline npf009n.o & 46.6 & 1.1124 & 0.0058 & 0.0116 & 1.124 \\
\hline npf010n.o & 51.9 & 1.1171 & 0.0052 & 0.0104 & 1.1275 \\
\hline npf011n.o & 58.2 & 1.108 & 0.006 & 0.012 & 1.12 \\
\hline npf012n.o & 76.6 & 1.105 & 0.0055 & 0.011 & 1.116 \\
\hline npf013n.o & 109.5 & 1.093 & 0.0051 & 0.0102 & 1.1032 \\
\hline npf014n.o & 186.4 & 1.0402 & 0.0053 & 0.0106 & 1.0508 \\
\hline
\end{tabular}


Figure 4.5.1-8. Two 5.25" $\mathrm{ID} \times 24$ " Tall Cylinders of UF 4 and Oil One Foot from a Wall $100 \%$ enrichment, 24 " concrete floor, 12 " concrete wall and ceiling, $\sim 100 \mathrm{~kg}$ water reflector simulating a person

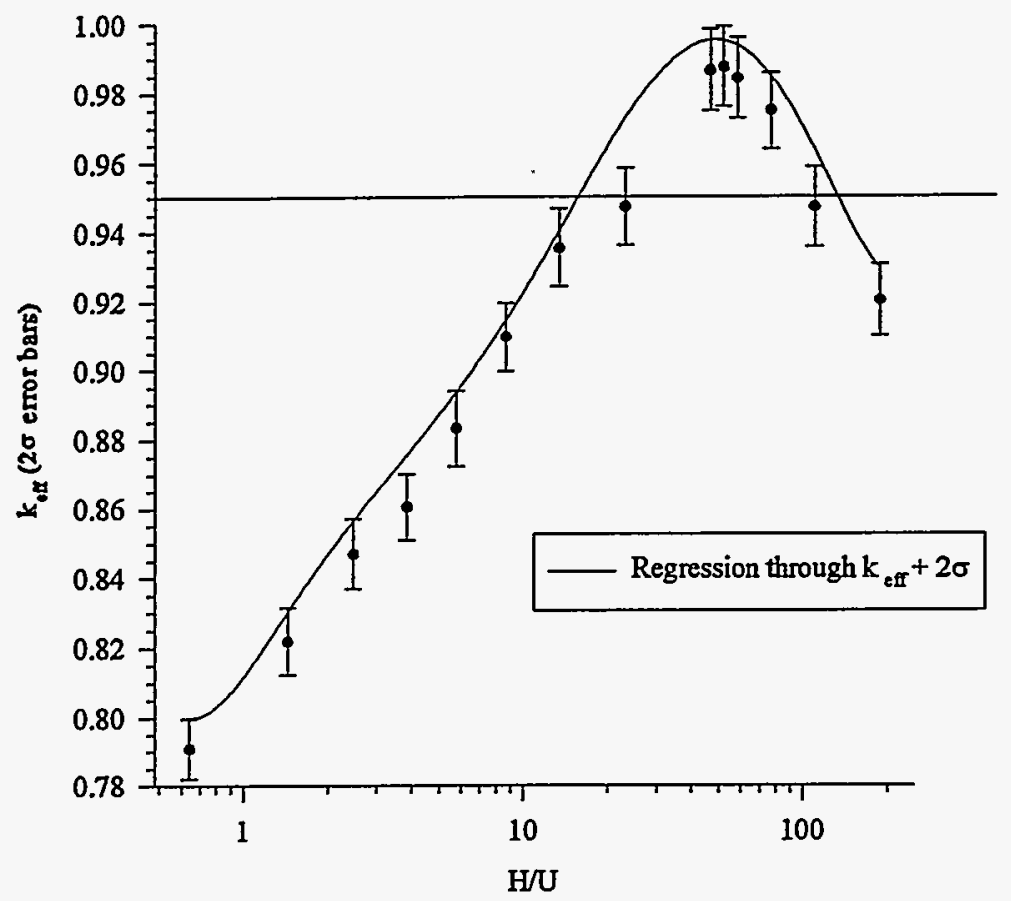

\begin{tabular}{|c|c|c|c|c|c|}
\hline \multicolumn{6}{|c|}{ Two 5.25 in. Diameter Tin/Steel Containers 1 ' from Concrete Wall } \\
\hline \multicolumn{6}{|c|}{ for $100 \%$ Enriched UF4+oil } \\
\hline Case & $\mathrm{H} / \mathrm{U}$ & Keff & K_sig & $2^{\star} \mathrm{K} \_$sig & Keff $+2^{\star} K$ sig \\
\hline apfoo1n.o & 0.64 & 0.7909 & 0.0044 & 0.0088 & 0.7997 \\
\hline apfoo2n.o & 1.44 & 0.822 & 0.0048 & 0.0096 & 0.8316 \\
\hline apfoo3n.o & 2.47 & 0.847 & 0.005 & 0.01 & 0.857 \\
\hline apfo04n.o & 3.84 & 0.8606 & 0.0048 & 0.0096 & 0.8702 \\
\hline apfoosn.o & 5.76 & 0.8832 & 0.0054 & 0.0108 & 0.894 \\
\hline apfo06n.o & 8.64 & 0.9095 & 0.005 & 0.01 & 0.9195 \\
\hline apf007n.o & 13.4 & 0.9354 & 0.0056 & 0.0112 & 0.9466 \\
\hline apfoosn.o & 23.1 & 0.9473 & 0.0055 & 0.011 & 0.9583 \\
\hline apfoo9n.o & 46.6 & 0.9863 & 0.0059 & 0.0118 & 0.9981 \\
\hline apfo10n.o & 51.9 & 0.9873 & 0.0058 & 0.0116 & 0.9989 \\
\hline apfo11n.o & 58.2 & 0.984 & 0.0058 & 0.0116 & 0.9956 \\
\hline apfo12n.o & 76.6 & 0.9747 & 0.0055 & 0.011 & 0.9857 \\
\hline apfo13n.o & 109.5 & 0.9471 & 0.0057 & 0.0114 & 0.9585 \\
\hline apf014n.o & 186.4 & 0.9202 & 0.0052 & 0.0104 & 0.9306 \\
\hline
\end{tabular}


Figure 4.5.1-9 Single 5.25" ID $\times 24^{\prime \prime}$ Tall Cylinder of $\mathrm{UF}_{4}$ and Oil $100 \%$ Enrichment, $24^{\prime \prime}$ concrete floor, $1^{\prime \prime}$ dose fit water reflector on all other sides, 12" ceiling $10^{\prime}$ above

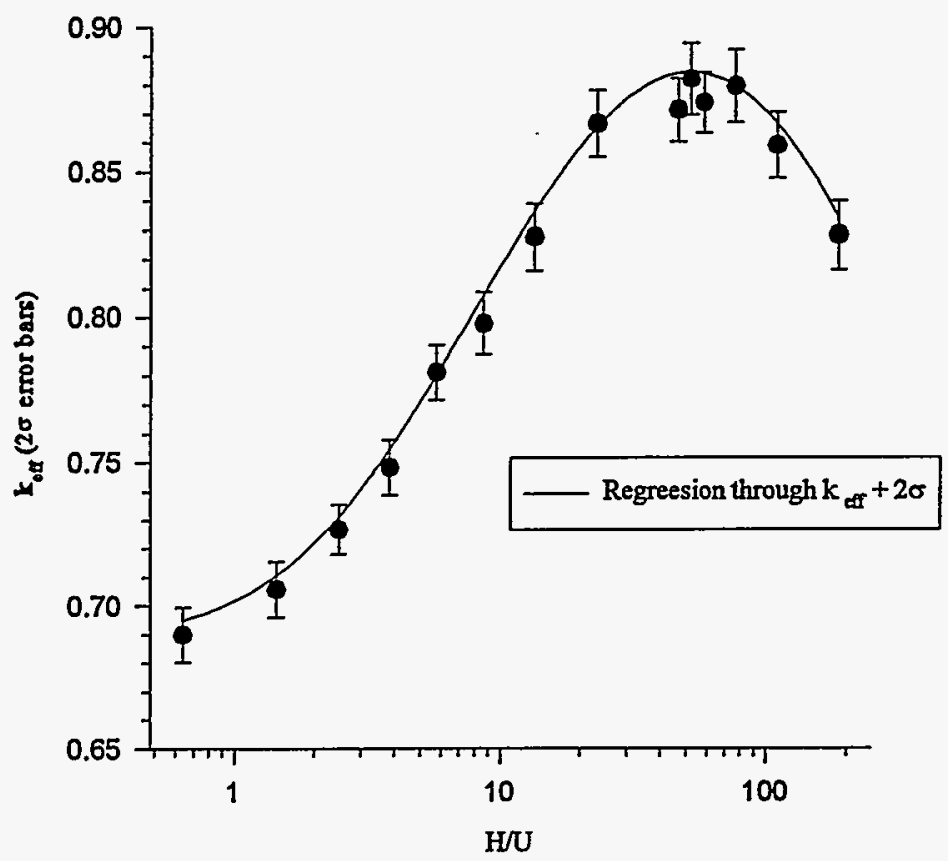

\begin{tabular}{|c|c|c|c|c|c|}
\hline \multicolumn{6}{|c|}{ Single 5.25" ID X 24" Tall Cylinder of UF4 and Oil } \\
\hline \multicolumn{6}{|c|}{$100 \%$ Enriched, 1" close fit water reflector } \\
\hline Case & $\mathrm{H} / \mathrm{U}$ & Keff & K_sig & $2^{*} \mathrm{~K}$ sig & Keff $+2 * K$ sig \\
\hline fullif-1.0p6 & 0.64 & 0.6899 & 0.0048 & 0.0096 & 0.6947 \\
\hline fulliff-1.144 & 1.44 & 0.7058 & 0.0049 & 0.0098 & 0.7107 \\
\hline fullif-1.247 & 2.47 & 0.7268 & 0.00433 & 0.00866 & 0.73113 \\
\hline fullirf-1.384 & 3.84 & 0.7483 & 0.00482 & 0.00964 & 0.75312 \\
\hline fullif-1.576 & 5.76 & 0.781 & 0.00471 & 0.00942 & 0.78571 \\
\hline fullrf-1.864 & 8.64 & 0.7977 & 0.00528 & 0.01056 & 0.80298 \\
\hline fullir-1.134 & 13.4 & 0.8272 & 0.00572 & 0.01144 & 0.83292 \\
\hline fullirf-1.231 & 23.1 & 0.8661 & 0.00578 & 0.01156 & 0.87188 \\
\hline fullif-1.466 & 46.6 & 0.8708 & 0.00547 & 0.01094 & 0.87627 \\
\hline fullif-1.519 & 51.9 & 0.8814 & 0.00621 & 0.01242 & 0.88761 \\
\hline fullrf-1.582 & 58.2 & 0.8733 & 0.00517 & 0.01034 & 0.87847 \\
\hline fullir-1.766 & 76.6 & 0.879 & 0.0063 & 0.0126 & 0.8853 \\
\hline fullir-1.109 & 109.5 & 0.8587 & 0.00565 & 0.0113 & 0.86435 \\
\hline fullif-1.186 & 186.4 & 0.8278 & 0.00587 & 0.01174 & 0.83367 \\
\hline
\end{tabular}


Figure 4.5.2-1. Single Fully Reflected 5.25" $\mathrm{ID} \times 24$ " Tall Cylinder of $\mathrm{UO}_{2} \mathrm{~F}_{2}$ $10 \%$ enrichment, 24" concrete foor, 12" (minimum) water rellector on all other sides

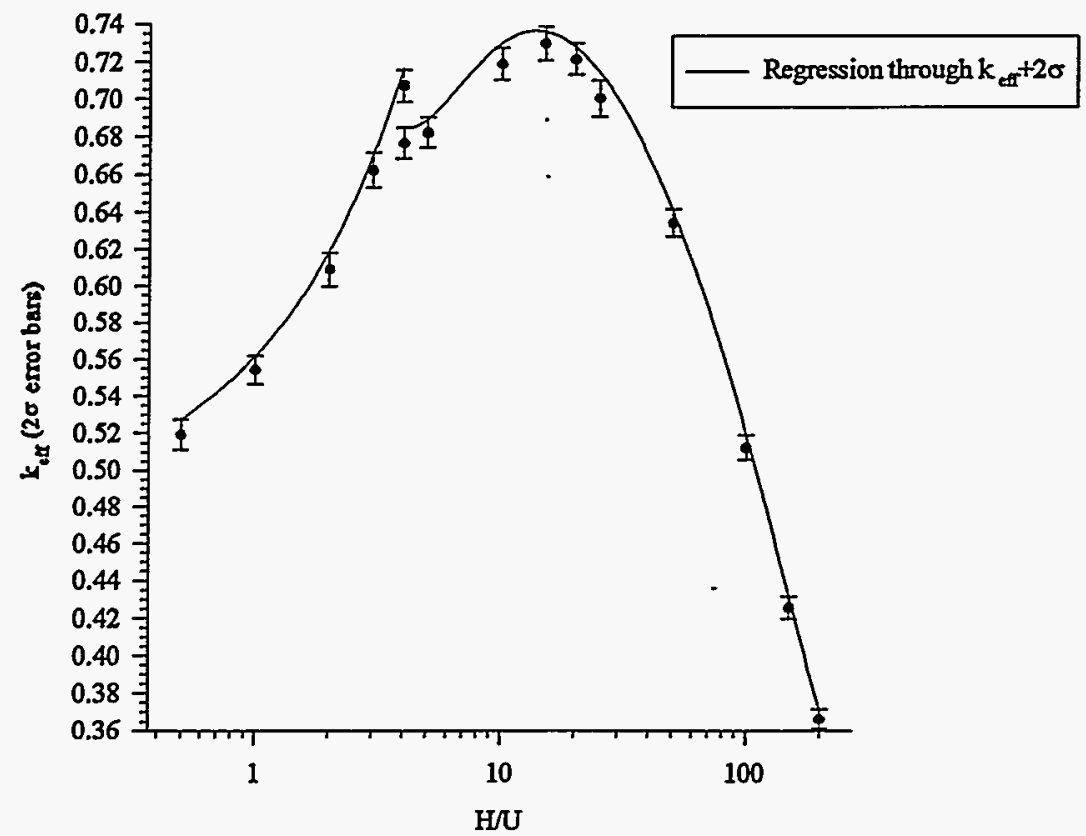

\begin{tabular}{|c|c|c|c|c|c|}
\hline \multicolumn{6}{|c|}{ for $10 \%$ Enriched UO2F $2+\mathrm{H} 20$} \\
\hline Case & H/U & Keff & K sig & $2 * K$ sig & Keff $+2^{\star} K$ sig \\
\hline frw001n.o & 0.5 & 0.5193 & 0.004 & 0.008 & 0.5273 \\
\hline frw002n.o & 1 & 0.5542 & 0.0039 & 0.0078 & 0.562 \\
\hline frw003n.o & 2 & 0.6088 & 0.0045 & 0.009 & 0.6178 \\
\hline frw004n.o & 3 & 0.6618 & 0.0047 & 0.0094 & 0.6712 \\
\hline frw005n.o & 3.99 & 0.7065 & 0.0043 & 0.0086 & 0.7151 \\
\hline frw006n.o & 4 & 0.6762 & 0.0041 & 0.0082 & 0.6844 \\
\hline frw007n.o & 5 & 0.6816 & 0.0039 & 0.0078 & 0.6894 \\
\hline frw008n.o & 10 & 0.7181 & 0.0043 & 0.0086 & 0.7267 \\
\hline frw009n.o & 15 & 0.7289 & 0.0044 & 0.0088 & 0.7377 \\
\hline frw010n.o & 20 & 0.7205 & 0.0042 & 0.0084 & 0.7289 \\
\hline frw011n.o & 25 & 0.6994 & 0.0049 & 0.0098 & 0.7092 \\
\hline frw012n.o & 50 & 0.6334 & 0.0036 & 0.0072 & 0.6406 \\
\hline frw013n.o & 100 & 0.5117 & 0.0033 & 0.0066 & 0.5183 \\
\hline frw014n.o & 150 & 0.4253 & 0.003 & 0.006 & 0.4313 \\
\hline frw015n.o & 200 & 0.3663 & 0.0026 & 0.0052 & 0.3715 \\
\hline
\end{tabular}


POEF-SH-30

\subsection{2 $10 \%$ Enriched $\mathrm{UO}_{2} \mathrm{~F}_{2}+\mathrm{H}_{2} \mathrm{O}$ and $\mathrm{UF}_{4}+\mathrm{Oil}$}

Table 4.5.2-0 gives the figure and table numbers that contain the results data for this container with various geometry models and fuel materials. The geometry model designators are explained in Table 4.1-1.

The three standard array cases were not analyzed because the $100 \%$ enriched standard array cases fell within the safety criterion. The maximum container poly thickness was considered in the TACW and TSCW cases for both $\mathrm{UO}_{2} \mathrm{~F}_{2}+\mathrm{H}_{2} \mathrm{O}$ and $\mathrm{UF}_{4}+$ oil. Polyethylene was used to model the F-Cans (constructed of steel coated with tin) in the TACW and TSCW cases to provide a measure of conservatism and to bound the smaller diameter containers with the analysis.

Table 4.5.2-0. Summary of References for F-Can Results for $10 \%$ Enrichment

\begin{tabular}{||c|c|c||}
\hline Geometry Model & Material & Figure/Table Number \\
\hline \hline SFR & $\mathrm{UO}_{2} \mathrm{~F}_{2}+\mathrm{H}_{2} \mathrm{O}$ & $4.5 .2-1$ \\
\hline $\mathrm{SCC}$ & $\mathrm{UO}_{2} \mathrm{~F}_{2}+\mathrm{H}_{2} \mathrm{O}$ & $4.5 .2-2$ \\
\hline TACW & $\mathrm{UO}_{2} \mathrm{~F}_{2}+\mathrm{H}_{2} \mathrm{O}$ & $4.5 .2-3$ \\
\hline $\mathrm{TSCW}$ & $\mathrm{UO}_{2} \mathrm{~F}_{2}+\mathrm{H}_{2} \mathrm{O}$ & $4.5 .2-4$ \\
\hline $\mathrm{SFR}$ & $\mathrm{UF}_{4}+\mathrm{oil}$ & $4.5 .2-5$ \\
\hline $\mathrm{SCC}$ & $\mathrm{UF}_{4}+\mathrm{oil}$ & $4.5 .2-6$ \\
\hline TACW & $\mathrm{UF}_{4}+\mathrm{oil}$ & $4.5 .2-7$ \\
\hline TSCW & $\mathrm{UF}_{4}+\mathrm{oil}$ & $4.5 .2-8$ \\
\hline
\end{tabular}


Figure 4.5.2-2. Single 5.25" ID $\times 24$ " Tall Cylinder of $\mathrm{UO}_{2} \mathrm{~F}_{2}$ in a Concrete Corner $10 \%$ enrichment, 24 " concrete floor, 12 " concrete walls and ceiling, $-100 \mathrm{~kg}$ water reflector simulating a person

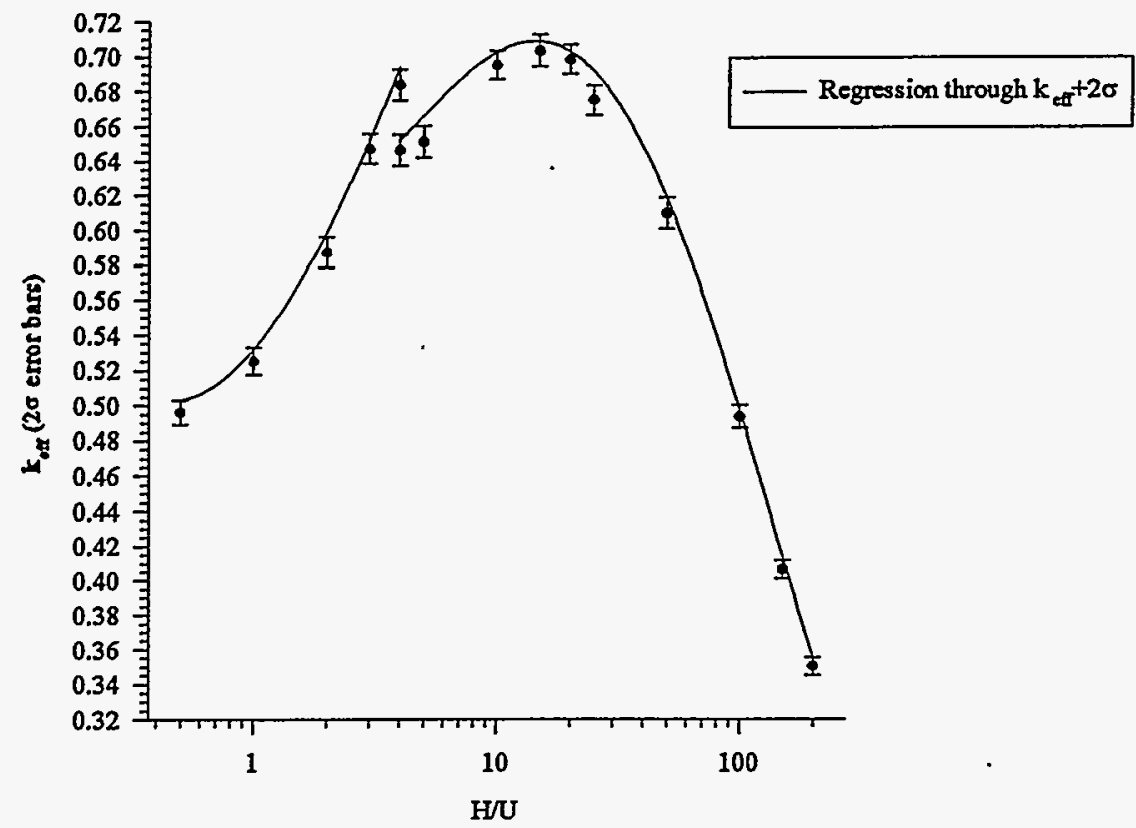

\begin{tabular}{|c|c|c|c|c|c|}
\hline \multicolumn{6}{|c|}{ Single 5.25 in. Diameter Tin Plated Steel Container in a Corner } \\
\hline \multicolumn{6}{|c|}{ for $10 \%$ Enriched UO2F2+H2O } \\
\hline Case & $H / U$ & Keff & K_sig & $2^{\star K} \mathrm{~K}$ sig & Keff $+2^{\star} K$ sig \\
\hline cpw001n.o & 0.5 & 0.4961 & 0.0035 & 0.007 & 0.5031 \\
\hline cpw002n.o & 1 & 0.5252 & 0.0039 & 0.0078 & 0.533 \\
\hline cpw003n.o & 2 & 0.5872 & 0.0044 & 0.0088 & 0.596 \\
\hline cpw004n.o & 3 & 0.6469 & 0.0043 & 0.0086 & 0.6555 \\
\hline cpw005n.o & 3.99 & 0.6834 & 0.0045 & 0.009 & 0.6924 \\
\hline cpw006n.o & 4. & 0.6462 & 0.0046 & 0.0092 & 0.6554 \\
\hline cpw007n.o & 5 & 0.651 & 0.0046 & 0.0092 & 0.6602 \\
\hline cpw008n.o & 10 & 0.6945 & 0.0041 & 0.0082 & 0.7027 \\
\hline cpw009n.o & 15 & 0.7027 & 0.0046 & 0.0092 & 0.7119 \\
\hline cpw010n.o & 20 & 0.6976 & 0.0042 & 0.0084 & 0.706 \\
\hline cpw011n.o & 25 & 0.6744 & 0.0042 & 0.0084 & 0.6828 \\
\hline cpw012n.o & 50 & 0.6088 & 0.0044 & 0.0088 & 0.6176 \\
\hline cpw013n.o & 100 & 0.4931 & 0.0032 & 0.0064 & 0.4995 \\
\hline cpw014n.o & 150 & 0.4061 & 0.0026 & 0.0052 & 0.4113 \\
\hline cpw015n.o & 200 & 0.3501 & 0.0026 & 0.0052 & 0.3553 \\
\hline
\end{tabular}


POEF-SH-30

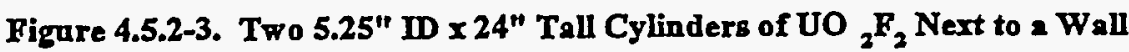

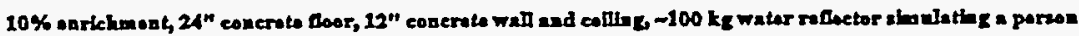

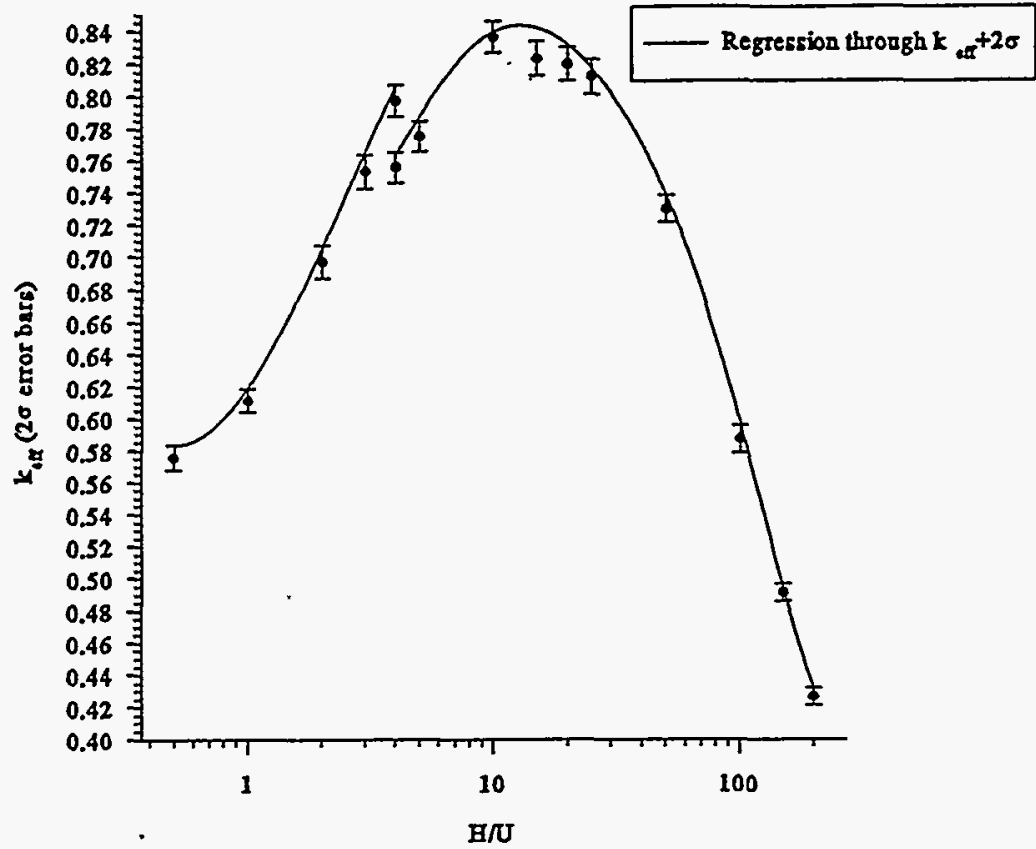

\begin{tabular}{|c|c|c|c|c|c|}
\hline \multicolumn{6}{|c|}{ for $10 \%$ Enriched UO2F2+H2O } \\
\hline Case & $\mathrm{H} / \mathrm{U}$ & Keff & K sig & $2^{\star} \hat{K}$ sig & Keff $+2^{\star} K$ sig \\
\hline d00n-525.0p5 & 0.5 & 0.5757 & 0.0038 & 0.0076 & 0.5833 \\
\hline$d 00 n-525.1$ & 1 & 0.6112 & 0.00365 & 0.0073 & 0.6185 \\
\hline d00n-525.2 & 2 & 0.6968 & 0.00505 & 0.0101 & 0.7069 \\
\hline doon-525.3 & 3 & 0.7526 & 0.00523 & 0.01046 & 0.76306 \\
\hline d00n-525.3p9 & 3.99 & 0.7968 & 0.00483 & 0.00966 & 0.80646 \\
\hline$d 00 n-525.4$ & 4 & 0.755 & 0.00478 & 0.00956 & 0.76456 \\
\hline d00n-525.5 & 5 & 0.7746 & 0.00462 & 0.00924 & 0.78384 \\
\hline d00n-525.10 & 10 & 0.8359 & 0.00486 & 0.00972 & 0.84562 \\
\hline doon-525.15 & 15 & 0.8228 & 0.00518 & 0.01036 & 0.83316 \\
\hline$d 00 n-525.20$ & 20 & 0.8193 & 0.00505 & 0.0101 & 0.8294 \\
\hline do0n-525.25 & 25 & 0.8115 & 0.0053 & 0.0106 & 0.8221 \\
\hline d00n-525.50 & 50 & 0.7294 & 0.00422 & 0.00844 & 0.73784 \\
\hline do0n-525.100 & 100 & 0.5865 & 0.00436 & 0.00872 & 0.59522 \\
\hline$d 00 n-525.150$ & 150 & 0.4913 & 0.00268 & 0.00536 & 0.49666 \\
\hline$d 00 n-525.200$ & 200 & 0.4266 & 0.00265 & 0.0053 & 0.4319 \\
\hline
\end{tabular}


Figure 4.5.2-4. Two 5.25" ID × 24" Tall Cylinders of $\mathrm{UO}_{2} \mathrm{~F}_{2}$ One Foot from a Wall

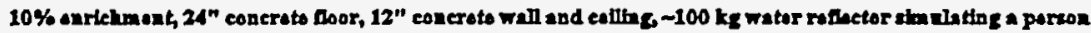

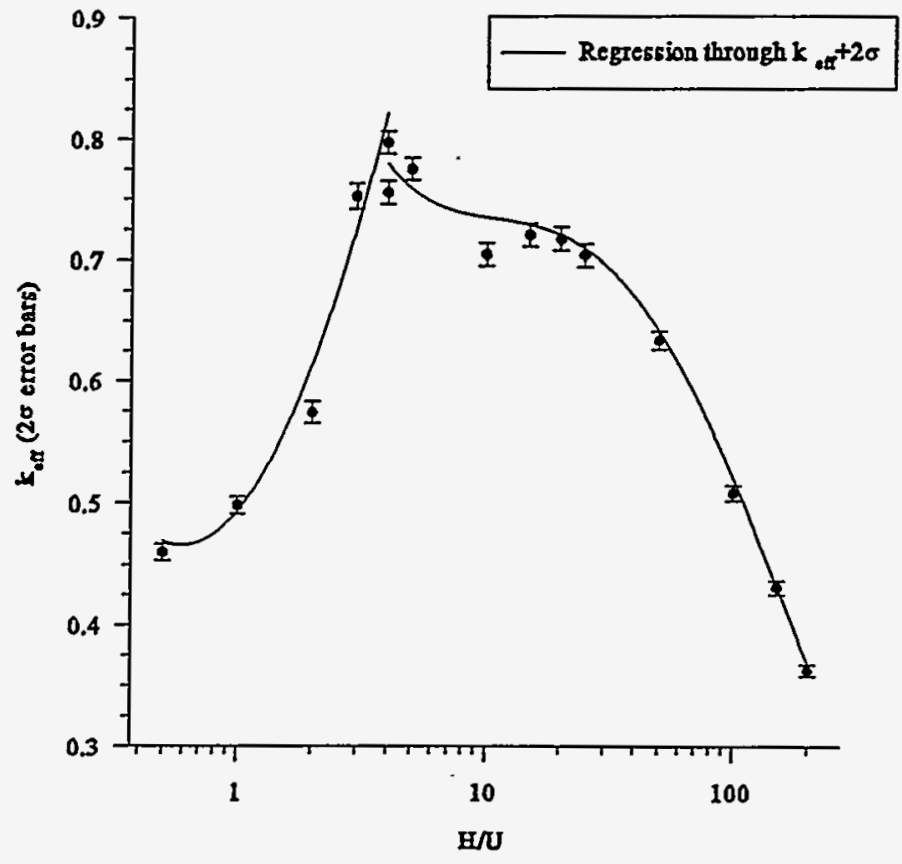

Two 5.25 in. Diameter Tin/Steel Containers 1 ' from Concrete Wall for $10 \%$ Enriched UO2F $2+\mathrm{H} 2 \mathrm{O}$

\begin{tabular}{|c|c|c|c|c|c|}
\hline Case & H/U & Keff & K sig & $2^{*}$ K sig & Keff+2* K sig \\
\hline$d 12 n-525.0 p 5$ & 0.5 & 0.4603 & 0.00342 & 0.00684 & 0.46714 \\
\hline$d 12 n-525.1$ & 1 & 0.4982 & 0.00347 & 0.00694 & 0.50514 \\
\hline$d 12 n-525.2$ & 2 & 0.5735 & 0.00444 & 0.00888 & 0.58238 \\
\hline$d 12 n-525.3$ & 3 & 0.7518 & 0.00523 & 0.01046 & 0.76226 \\
\hline$d 12 n-525.3 p 9$ & 3.99 & 0.7968 & 0.00483 & 0.00966 & 0.80646 \\
\hline$d 12 n-525.4$ & 4 & 0.755 & 0.00478 & 0.00956 & 0.76456 \\
\hline$d 12 n-525.5$ & 5 & 0.7746 & 0.00462 & 0.00924 & 0.78384 \\
\hline$d 12 n-525.10$ & 10 & 0.704 & 0.00478 & 0.00956 & 0.71356 \\
\hline$d 12 n-525.15$ & 15 & 0.7201 & 0.00472 & 0.00944 & 0.72954 \\
\hline$d 12 n-525.20$ & 20 & 0.7167 & 0.0049 & 0.0098 & 0.7265 \\
\hline$d 12 n-525.25$ & 25 & 0.7034 & 0.00456 & 0.00912 & 0.71252 \\
\hline$d 12 n-525.50$ & 50 & 0.6332 & 0.00383 & 0.00766 & 0.64086 \\
\hline$d 12 n-525.100$ & 100 & 0.5078 & 0.00304 & 0.00608 & 0.51388 \\
\hline$d 12 n-525.150$ & 150 & 0.4309 & 0.00293 & 0.00586 & 0.43676 \\
\hline$d 12 n-525.200$ & 200 & 0.3623 & 0.00236 & 0.00472 & 0.36702 \\
\hline
\end{tabular}


Figure 4.5.2-5. Single Fully Reflected 5.25" ID $\times$ 24" Tall Cylinder of UF , and Oil 10\% enrichment, 24" concroto floor, 12" (minimam) water rebector an all other zides

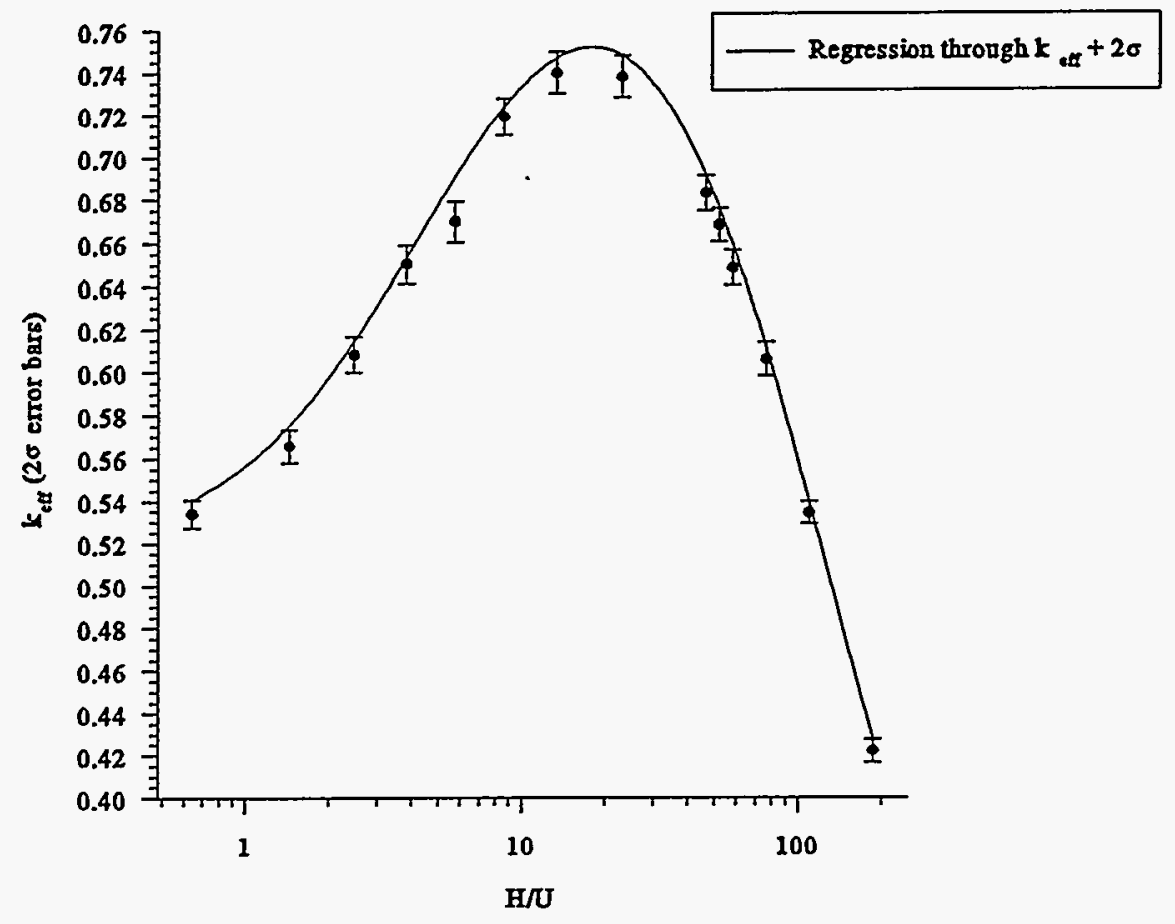

\begin{tabular}{|c|c|c|c|c|c|}
\hline \multicolumn{6}{|c|}{ for $10 \%$ Enriched UF4+oil } \\
\hline Case & $\mathrm{H} / \mathrm{U}$ & Keff & K_sig & $2^{*} \mathrm{~K}$ sig & Keff $+2^{\star} \mathrm{K}$ sig \\
\hline fr-525.p65.o & 0.65 & 0.5344 & 0.004 & 0.008 & 0.5424 \\
\hline fr -525.145 .0 & 1.45 & 0.57 & 0.0041 & 0.0082 & 0.5782 \\
\hline fr-525.249.0 & 2.49 & 0.6011 & 0.0043 & 0.0086 & 0.6097 \\
\hline$f r-525.388 .0$ & 3.88 & 0.6436 & 0.0041 & 0.0082 & 0.6518 \\
\hline$f r-525.582 .0$ & 5.82 & 0.6722 & 0.0046 & 0.0092 & 0.6814 \\
\hline$f r-525.873 .0$ & 8.73 & 0.7216 & 0.0049 & 0.0098 & 0.7314 \\
\hline fr-525.136.o & 13.6 & 0.7472 & 0.0045 & 0.009 & 0.7562 \\
\hline$f r-525.233 .0$ & 23.3 & 0.7388 & 0.0042 & 0.0084 & 0.7472 \\
\hline fr-525.471.o & 47.1 & 0.6783 & 0.004 & 0.008 & 0.6863 \\
\hline$f r-525.524 .0$ & 52.4 & 0.6699 & 0.0037 & 0.0074 & 0.6773 \\
\hline fr-525.588.o & 58.8 & 0.6621 & 0.0039 & 0.0078 & 0.6699 \\
\hline$f r-525.773 .0$ & 77.3 & 0.6152 & 0.0038 & 0.0076 & 0.6228 \\
\hline fr-525.110.o & 110.6 & 0.529 & 0.0033 & 0.0066 & 0.5356 \\
\hline fr-525.188.o & 188.2 & 0.4163 & 0.0024 & 0.0048 & 0.4211 \\
\hline
\end{tabular}


Figure 4.5.2-6. Single 5.25" ID $\times 24 "$ Tall Cylinder of UF , and Oil in a Concrete Corner

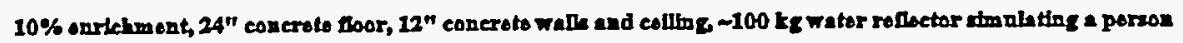

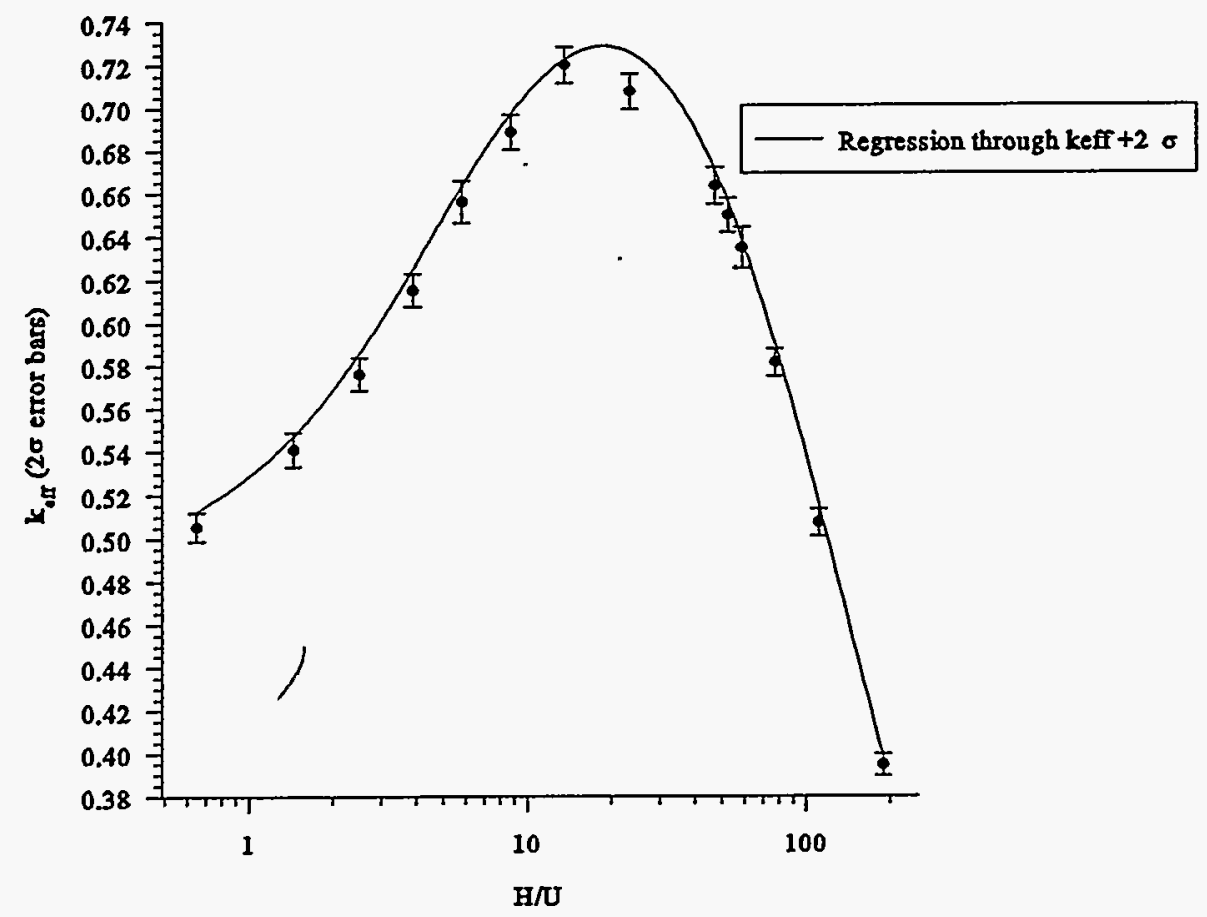

\begin{tabular}{|c|c|c|c|c|c|}
\hline \multicolumn{6}{|c|}{ Single 5.25 in. Diameter Tin/Steel Container in a Corner } \\
\hline \multicolumn{6}{|c|}{ for $10 \%$ Enriched UF4+oil } \\
\hline Case & $\mathrm{H} / \mathrm{U}$ & Keff & K sig & $2 * K$ sig & Keff $+2 *$ K_sig \\
\hline cr-525.p65.0 & 0.65 & 0.5053 & 0.0033 & 0.0066 & 0.5119 \\
\hline cr-525.145.0 & 1.45 & 0.5409 & 0.0039 & 0.0078 & 0.5487 \\
\hline cr-525.249.o & 2.49 & 0.5758 & 0.0039 & 0.0078 & 0.5836 \\
\hline cr-525.388.0 & 3.88 & 0.6153 & 0.0038 & 0.0076 & 0.6229 \\
\hline cr-525.582.0 & 5.82 & 0.6557 & 0.0049 & 0.0098 & 0.6655 \\
\hline cr-525.873.o & 8.73 & 0.6882 & 0.0041 & 0.0082 & 0.6964 \\
\hline cr-525.136.o & 13.6 & 0.7197 & 0.0042 & 0.0084 & 0.7281 \\
\hline cr-525.233.o & 23.3 & 0.7072 & 0.0042 & 0.0084 & 0.7156 \\
\hline cr-525.471.o & 47.1 & 0.6628 & 0.0043 & 0.0086 & 0.6714 \\
\hline cr-525.524.0 & 52.4 & 0.649 & 0.0039 & 0.0078 & 0.6568 \\
\hline cr-525.588.0 & 58.8 & 0.6343 & 0.0046 & 0.0092 & 0.6435 \\
\hline cr-525.773.o & 77.3 & 0.581 & 0.0032 & 0.0064 & 0.5874 \\
\hline cr-525.110.0 & 110.6 & 0.507 & 0.0031 & 0.0062 & 0.5132 \\
\hline cr-525.188.0 & 188.2 & 0.3945 & 0.0025 & 0.005 & 0.3995 \\
\hline
\end{tabular}


Figure 4.5.2-7. Two 5.25" ID $~$ 24" Tall Cylinders of UF 4 and Oil Nert to a Wall

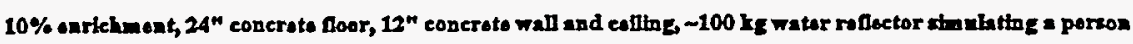

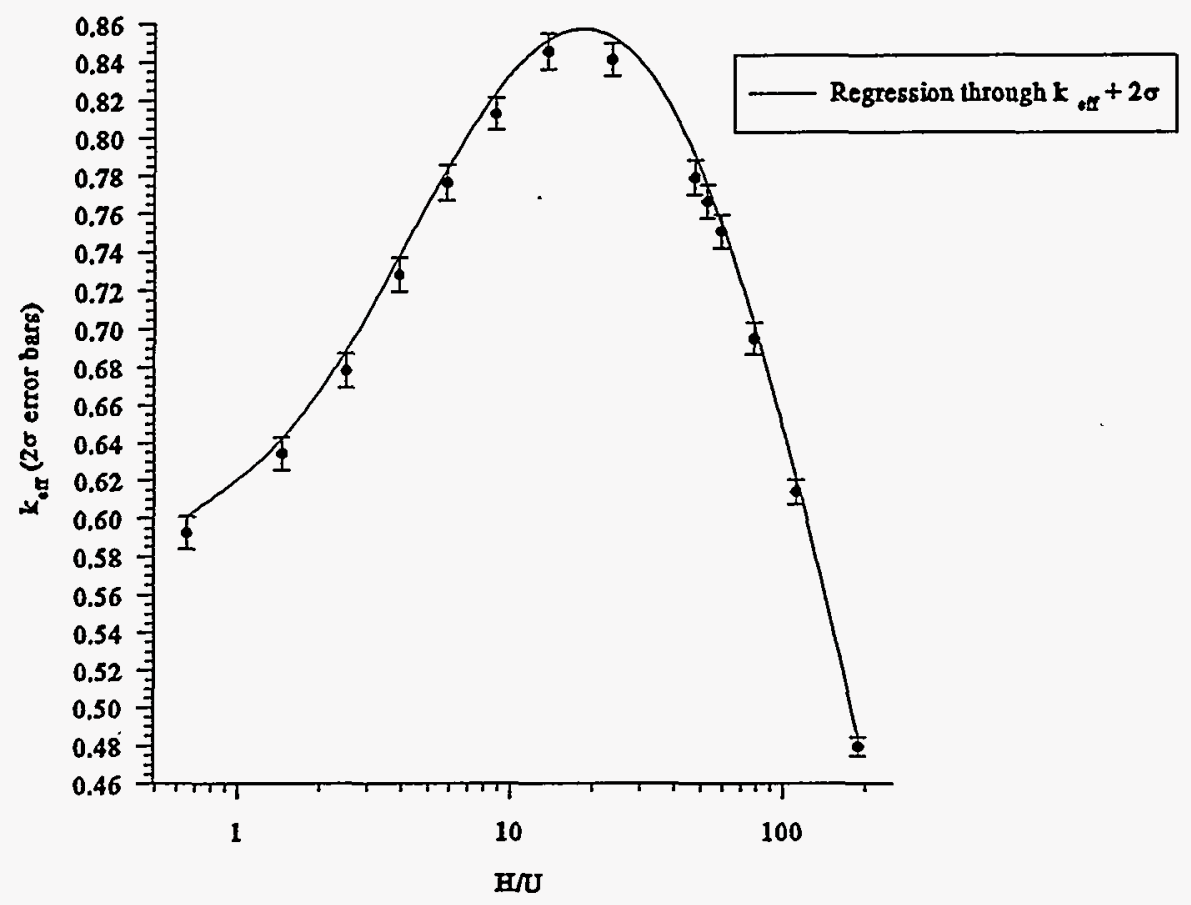

Two 5.25 in. Dia. Tin/Steel Containers Adjacent to Concrete Wall for $10 \%$ Enriched UF4+oil

\begin{tabular}{|c|c|c|c|c|c|}
\hline Case & H/U & Keff & K sig & $2^{*}$ K sig & Keff $+2^{*}$ K sig \\
\hline d00n-525.p65 & 0.65 & 0.5923 & 0.00429 & 0.00858 & 0.60088 \\
\hline d00n-525.145 & 1.45 & 0.6342 & 0.00453 & 0.00906 & 0.64326 \\
\hline d00n-525.249 & 2.49 & 0.6783 & 0.00453 & 0.00906 & 0.68736 \\
\hline d00n-525.388 & 3.88 & 0.728 & 0.0044 & 0.0088 & 0.7368 \\
\hline$d 00 n-525.582$ & 5.82 & 0.7761 & 0.0046 & 0.0092 & 0.7853 \\
\hline$d 00 n-525.873$ & 8.73 & 0.8123 & 0.00416 & 0.00832 & 0.82062 \\
\hline$d 00 n-525.136$ & 13.6 & 0.8448 & 0.0048 & 0.0096 & 0.8544 \\
\hline$d 00 n-525.233$ & 23.3 & 0.8404 & 0.00427 & 0.00854 & 0.84894 \\
\hline$d 00 n-525.471$ & 47.1 & 0.7785 & 0.0045 & 0.009 & 0.7875 \\
\hline$d 00 n-525.524$ & 52.4 & 0.766 & 0.00438 & 0.00876 & 0.77476 \\
\hline$d 00 n-525.588$ & 58.8 & 0.7499 & 0.00439 & 0.00878 & 0.75868 \\
\hline$d 00 n-525.773$ & 77.3 & 0.6946 & 0.00402 & 0.00804 & 0.70264 \\
\hline$d 00 n-525.110$ & 110.6 & 0.6133 & 0.00318 & 0.00636 & 0.61966 \\
\hline$d 00 n-525.188$ & 188.2 & 0.4796 & 0.00239 & 0.00478 & 0.48438 \\
\hline
\end{tabular}


Figure 4.5.2-8. Two 5.25" $\mathrm{D} \times 24$ " Tall Cylinders of UF , and Oil One Foot from a Wall

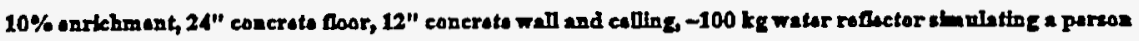

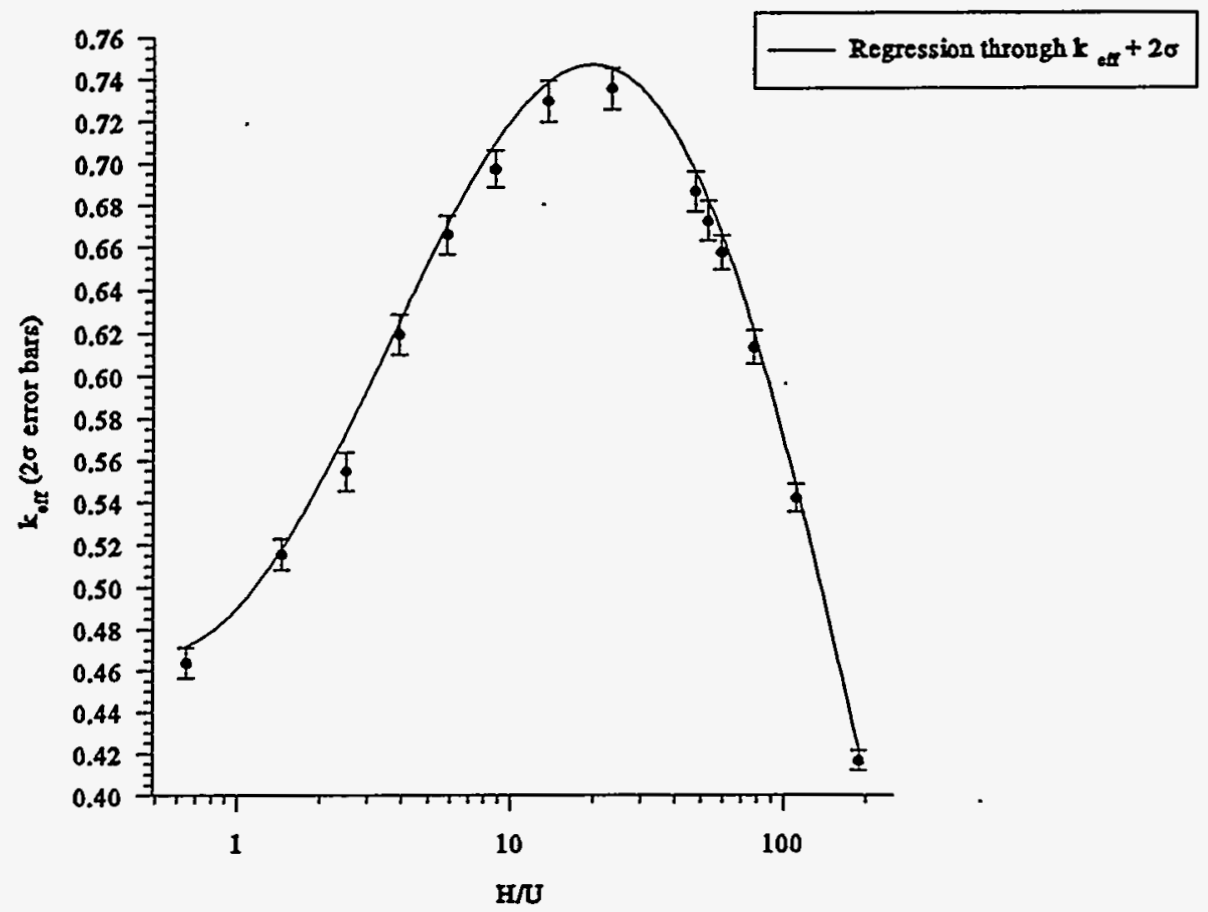

\begin{tabular}{|c|c|c|c|c|c|}
\hline \multicolumn{6}{|c|}{ for $10 \%$ Enriched UF4+oil } \\
\hline Case & $\mathrm{H} / \mathrm{U}$ & Keff & K_sig & $2^{\star} \mathrm{K}$ sig & Keff $+2 * K$ sig \\
\hline$d 12 n-525 . p 65$ & 0.65 & 0.4639 & 0.00372 & 0.00744 & 0.47134 \\
\hline d12n-525.145 & 1.45 & 0.5156 & 0.0036 & 0.0072 & 0.5228 \\
\hline d12n-525.249 & 2.49 & 0.5547 & 0.00468 & 0.00936 & 0.56406 \\
\hline d12n-525.388 & 3.88 & 0.6193 & 0.00466 & 0.00932 & 0.62862 \\
\hline d12n-525.582 & 5.82 & 0.6657 & 0.0045 & 0.009 & 0.6747 \\
\hline$d 12 n-525.873$ & 8.73 & 0.697 & 0.00441 & 0.00882 & 0.70582 \\
\hline $\mathrm{d} 12 \mathrm{n}-525.136$ & 13.6 & 0.7292 & 0.00483 & 0.00966 & 0.73886 \\
\hline$d 12 n-525.233$ & 23.3 & 0.7352 & 0.00489 & 0.00978 & 0.74498 \\
\hline d12n-525.471 & 47.1 & 0.6861 & 0.00477 & 0.00954 & 0.69564 \\
\hline $\mathrm{d} 12 \mathrm{n}-525.524$ & 52.4 & 0.6719 & 0.00475 & 0.0095 & 0.6814 \\
\hline $\mathrm{d} 12 \mathrm{n}-525.588$ & 58.8 & 0.6569 & 0.004 & 0.008 & 0.6649 \\
\hline$d 12 n-525.773$ & 77.3 & 0.6134 & 0.00396 & 0.00792 & 0.62132 \\
\hline$d 12 n-525.110$ & 110.6 & 0.5417 & 0.00328 & 0.00656 & 0.54826 \\
\hline$d 12 n-525.188$ & 188.2 & 0.4167 & 0.00243 & 0.00486 & 0.42156 \\
\hline
\end{tabular}


POEF-SH-30

\subsection{25-inch-Diameter by 15-inch Tin Can}

This section presents results of calculations designed to model the Z-Can containers described in Table 1.0-1. Table 4.6-0 gives the figure and table numbers that contain the geometry models and fuel materials. The results of the SFR cases with $\mathrm{UO}_{2} \mathrm{~F}_{2}$ and $\mathrm{U}_{3} \mathrm{O}_{8}$, demonstrates that $\mathrm{U}_{3} \mathrm{O}_{8}$ is only slightly more reactive. For this reason, the full range of geometry models is only analyzed for the $\mathrm{U}_{3} \mathrm{O}_{8}$ cases. The array in the each of the array models is moved to 1 -foot from the concrete wall enclosure to support a less restrictive array position. The geometry model designators are explained in Table 4.1-1.

Table 4.6-0. Summary of References for Z-Can Results

\begin{tabular}{|c|c|c|}
\hline Geometry Model & Material & Figure/Table Number \\
\hline \hline SFR & $\mathrm{UO}_{2} \mathrm{~F}_{2}$ & $4.6-1$ \\
\hline SFR & $\mathrm{UF}_{4}+$ oil & $4.6-2$ \\
\hline TSCW & $\mathrm{UO}_{2} \mathrm{~F}_{2}$ & $4.6-3$ \\
\hline SMRC & $\mathrm{UF}_{4}+\mathrm{oil}$ & $4.6-4$ \\
\hline SFR & $\mathrm{U}_{3} \mathrm{O}_{8}$ & $4.6-5$ \\
\hline SCC & $\mathrm{U}_{3} \mathrm{O}_{8}$ & $4.6-6$ \\
\hline TACW & $\mathrm{U}_{3} \mathrm{O}_{8}$ & $4.6-7$ \\
\hline TSCW & $\mathrm{U}_{3} \mathrm{O}_{8}$ & $4.6-8$ \\
\hline ACE* & $\mathrm{U}_{3} \mathrm{O}_{8}$ & $4.6-9$ \\
\hline AI* & $\mathrm{U}_{3} \mathrm{O}_{8}$ & $4.6-10$ \\
\hline AOSP* & $\mathrm{U}_{3} \mathrm{O}_{8}$ & $4.6-11$ \\
\hline
\end{tabular}

* Array positioned 1-foot from concrete enclosure. 
Figure 4.6-1. Single Fully Reflected 5.25" ID $\times 15^{\prime \prime}$ Tall Cylinder of UO ${ }_{2} F_{2}$

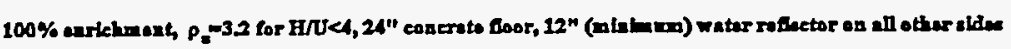

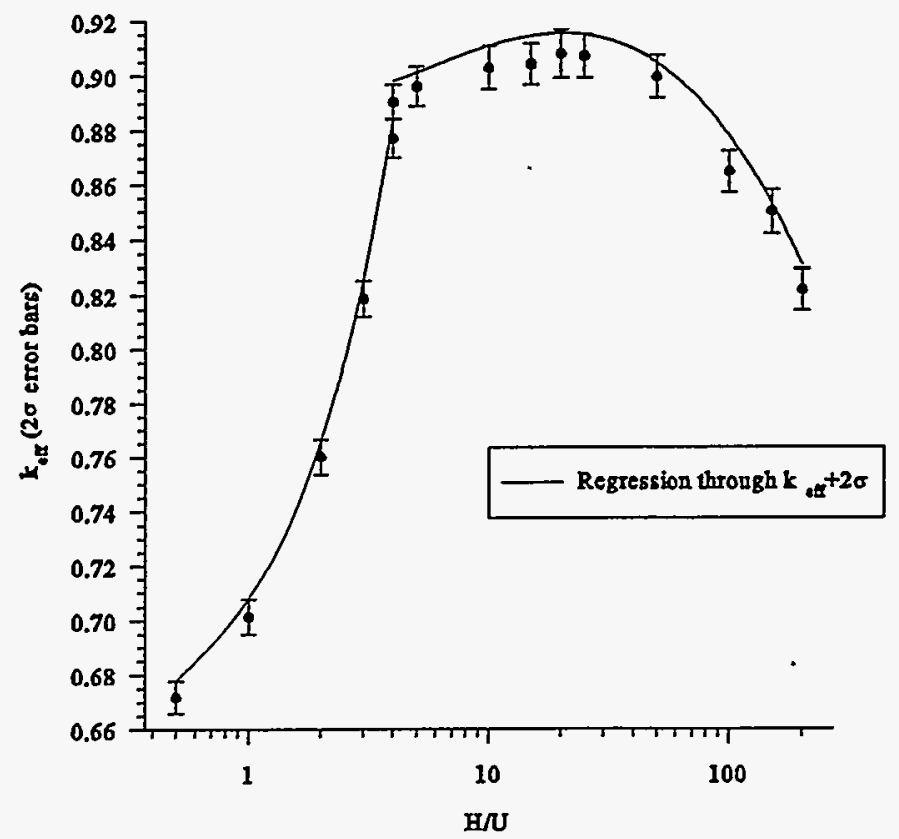

Single Fully Reflected 5.25-in. by 15-in. Tall Cylinder of UO2F2 For $100 \%$ Enrichment

\begin{tabular}{|c|c|c|c|c|c|}
\hline Case & H/U & Keff & K sig & $2^{\star}$ K_sig & Keff $+2^{\star}$ K_sig \\
\hline fr-zcan01n.o & 0.5 & 0.6718 & 0.0029 & 0.0058 & 0.6776 \\
\hline fr-zcan02n.o & 1 & 0.7013 & 0.0032 & 0.0064 & 0.7077 \\
\hline fr-zcan03n.o & 2 & 0.7599 & 0.0032 & 0.0064 & 0.7663 \\
\hline fr-zcan04n.o & 3 & 0.8185 & 0.0032 & 0.0064 & 0.8249 \\
\hline fr-zcan05n.o & 3.99 & 0.877 & 0.0035 & 0.007 & 0.884 \\
\hline fr-zcan06n.o & 4 & 0.8902 & 0.0032 & 0.0064 & 0.8966 \\
\hline fr-zcan07n.o & 5 & 0.8959 & 0.0036 & 0.0072 & 0.9031 \\
\hline fr-zcan08n.o & 10 & 0.9026 & 0.0039 & 0.0078 & 0.9104 \\
\hline fr-zcan09n.o & 15 & 0.9039 & 0.0037 & 0.0074 & 0.9113 \\
\hline fr-zcan10n.o & 20 & 0.9075 & 0.0043 & 0.0086 & 0.9161 \\
\hline fr-zcan11n.o & 25 & 0.9067 & 0.0039 & 0.0078 & 0.9145 \\
\hline fr-zcan12n.o & 50 & 0.8992 & 0.0039 & 0.0078 & 0.907 \\
\hline fr-zcan13n.o & 100 & 0.8643 & 0.0039 & 0.0078 & 0.8721 \\
\hline fr-zcan14n.o & 150 & 0.8495 & 0.004 & 0.008 & 0.8575 \\
\hline fr-zcan15n.o & 200 & 0.8214 & 0.0037 & 0.0074 & 0.8288 \\
\hline
\end{tabular}


Figure 4.6-2. Single Fully Reflected 5.25" ID $\times 15$ "Tall Cylinder of UF , and Oil

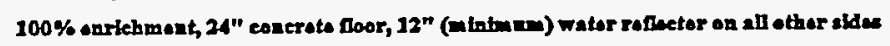

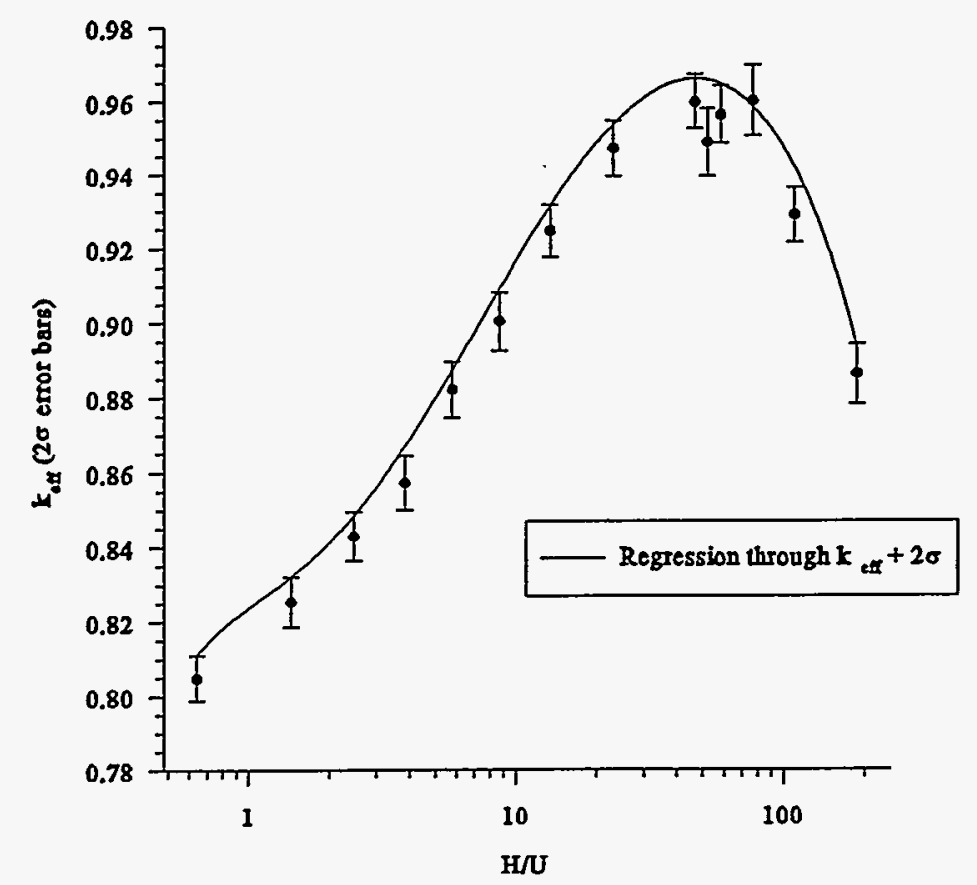

\begin{tabular}{|c|c|c|c|c|c|}
\hline \multicolumn{6}{|c|}{ Single 5.25 -in. ID by 15 -in. Tall Cylinder of UF4+oil } \\
\hline \multicolumn{6}{|c|}{ Full reflection, $100 \%$ Enrichment } \\
\hline Case & $\mathrm{H} / \mathrm{U}$ & Keff & K_sig & $2^{\star} \mathrm{K}$ sig & Keff $+2^{\star} \mathrm{K}$ sig \\
\hline fr-zcan16n.o & 0.64 & 0.8049 & 0.0031 & 0.0062 & 0.8111 \\
\hline fr-zcan17n.o & 1.44 & 0.8253 & 0.0034 & 0.0068 & 0.8321 \\
\hline fr-zcan $18 n . o$ & 2.47 & 0.8429 & 0.0033 & 0.0066 & 0.8495 \\
\hline fr-zcan19n.o & 3.84 & 0.8571 & 0.0036 & 0.0072 & 0.8643 \\
\hline $\mathrm{fr}-\mathrm{zcan} 20 \mathrm{n} . \mathrm{o}$ & 5.76 & 0.882 & 0.0037 & 0.0074 & 0.8894 \\
\hline fr-zcan21n.o & 8.64 & 0.9002 & 0.0039 & 0.0078 & 0.908 \\
\hline fr-zcan22n.o & 13.4 & 0.9243 & 0.0036 & 0.0072 & 0.9315 \\
\hline fr-zcan23n.o & 23.1 & 0.9467 & 0.0038 & 0.0076 & 0.9543 \\
\hline fr-zcan24n.o & 46.6 & 0.9593 & 0.0036 & 0.0072 & 0.9665 \\
\hline fr-zcan25n.o & 51.9 & 0.9483 & 0.0046 & 0.0092 & 0.9575 \\
\hline fr-zcan26n.o & 58.2 & 0.9559 & 0.0039 & 0.0078 & 0.9637 \\
\hline $\mathrm{fr}-\mathrm{zcan} 27 \mathrm{n} . \mathrm{o}$ & 76.6 & 0.9596 & 0.0047 & 0.0094 & 0.969 \\
\hline $\mathrm{fr}-\mathrm{zcan} 28 \mathrm{n} . \mathrm{o}$ & 109.5 & 0.9285 & 0.0037 & 0.0074 & 0.9359 \\
\hline fr-zcan29n.o & 186.4 & 0.886 & 0.004 & 0.008 & 0.894 \\
\hline
\end{tabular}


Figare 4.6-3. Two 5.25" ID $\times 15$ "Tall Cylinders of UO ${ }_{2} F_{2}$ One Foot from a Wall

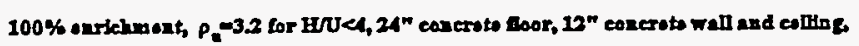
$-100 \mathrm{~kg}$ watur roflector aba rlatting a parso

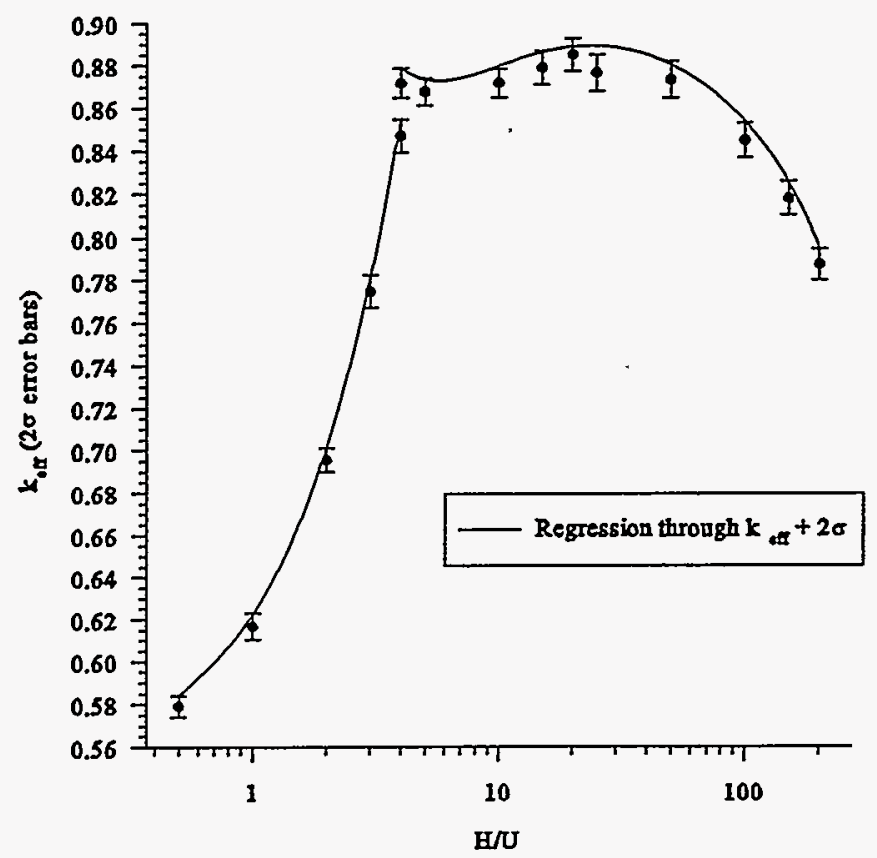

\begin{tabular}{|c|c|c|c|c|c|}
\hline \multicolumn{6}{|c|}{ Two 5.25-in. ID by 15-in. Tall Cylinders of UO2F2 1' from a Wall For 100\% Enrichment } \\
\hline Case & H/U & Keff & K sig & $2^{*} \mathrm{~K}$ sig & Keff+2*K sig \\
\hline ap-zcan01n.o & 0.5 & 0.5792 & 0.0024 & 0.0048 & 0.584 \\
\hline ap-zcan02n.o & 1 & 0.6168 & 0.0031 & 0.0062 & 0.623 \\
\hline ap-zcan03n.o & 2 & 0.6953 & 0.0027 & 0.0054 & 0.7007 \\
\hline ap-zcan04n.o & 3 & 0.7745 & 0.0038 & 0.0076 & 0.7821 \\
\hline ap-zcan05n.o & 3.99 & 0.8464 & 0.0038 & 0.0076 & 0.854 \\
\hline ap-zcan06n.o & 4 & 0.8711 & 0.0035 & 0.007 & 0.8781 \\
\hline ap-zcan07n.o & 5 & 0.8671 & 0.0032 & 0.0064 & 0.8735 \\
\hline ap-zcan08n.o & 10 & 0.8711 & 0.0033 & 0.0066 & 0.8777 \\
\hline ap-zcan09n.o & 15 & 0.8782 & 0.0039 & 0.0078 & 0.886 \\
\hline ap-zcan10n.o & 20 & 0.8843 & 0.0038 & 0.0076 & 0.8919 \\
\hline ap-zcan11n.o & 25 & 0.8756 & 0.0042 & 0.0084 & 0.884 \\
\hline ap-zcan12n.o & 50 & 0.8723 & 0.0042 & 0.0084 & 0.8807 \\
\hline ap-zcan13n.o & 100 & 0.844 & 0.0041 & 0.0082 & 0.8522 \\
\hline ap-zcan14n.o & 150 & 0.8171 & 0.0039 & 0.0078 & 0.8249 \\
\hline ap-zcan15n.o & 200 & 0.7869 & 0.0036 & 0.0072 & 0.7941 \\
\hline
\end{tabular}


Figure 4.6-4. Single 5.25" $\mathrm{m} \times 15$ " Tall Cylinder of $\mathrm{UF}$, and Oil $100 \%$ anriehmont, 24" concrete floor, 1" close fit water reflector on all other wides, 12 " celling $10^{\prime}$ above

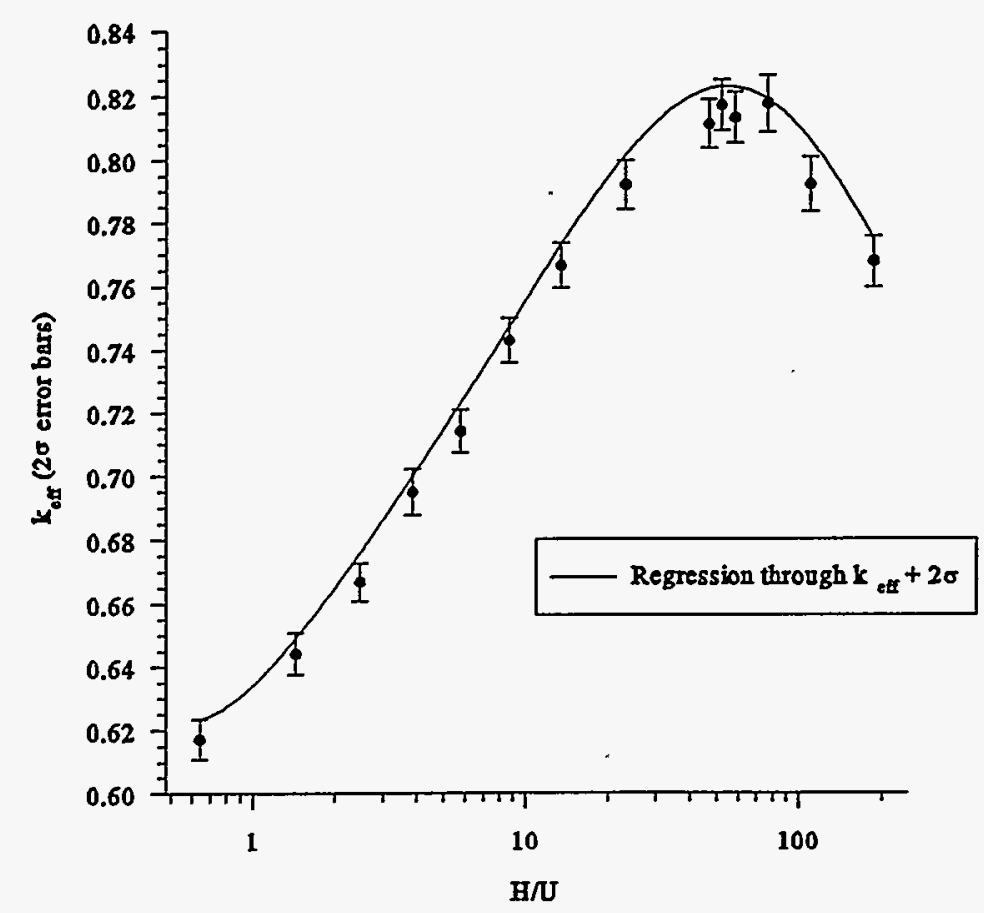

\begin{tabular}{|c|c|c|c|c|c|}
\hline \multicolumn{6}{|c|}{ Single 5.25 -in. ID by 15 -in. Tall Cylinder of UF4+Oil 1 " water reflector } \\
\hline \multicolumn{6}{|c|}{ For $100 \%$ Enrichment } \\
\hline Case & $\mathrm{H} / \mathrm{U}$ & Keff & K sig & $2^{*} \mathrm{~K} \_$sig & Keff $+2^{\star} K$ sig \\
\hline cyzcan01n.o & 0.64 & 0.6171 & 0.0031 & 0.0062 & 0.6233 \\
\hline cyzcan $02 n .0$ & 1.44 & 0.6442 & 0.0032 & 0.0064 & 0.6506 \\
\hline cyzcan $03 n .0$ & 2.47 & 0.6668 & 0.003 & 0.006 & 0.6728 \\
\hline cyzcan04n.o & 3.84 & 0.6948 & 0.0036 & 0.0072 & 0.702 \\
\hline cyzcan $05 n .0$ & 5.76 & 0.7139 & 0.0034 & 0.0068 & 0.7207 \\
\hline cyzcan06n.o & 8.64 & 0.7427 & 0.0035 & 0.007 & 0.7497 \\
\hline cyzcan07n.o & 13.4 & 0.7661 & 0.0035 & 0.007 & 0.7731 \\
\hline cyzcan08n.o & 23.1 & 0.7917 & 0.0038 & 0.0076 & 0.7993 \\
\hline cyzcan09n.o & 46.6 & 0.8109 & 0.0038 & 0.0076 & 0.8185 \\
\hline cyzcan 10 n.o & 51.9 & 0.8168 & 0.0039 & 0.0078 & 0.8246 \\
\hline cyzcan $11 n .0$ & 58.2 & 0.8129 & 0.004 & 0.008 & 0.8209 \\
\hline cyzcan 12 n.o & 76.6 & 0.8172 & 0.0044 & 0.0088 & 0.826 \\
\hline cyzcan $13 n .0$ & 109.5 & 0.7918 & 0.0043 & 0.0086 & 0.8004 \\
\hline cyzcan 14 n.o & 186.4 & 0.7674 & 0.004 & 0.008 & 0.7754 \\
\hline
\end{tabular}


Figure 4.6-5. Single Fully Reflected 5.25" ID $\times 15^{\prime \prime}$ Tall Cylinder of $\mathrm{U}_{3} \mathrm{O}_{8}$ $100 \%$ Barichmont, 24" concrete Loor, 12" (ninimans) wator rolloctor on all othor sides

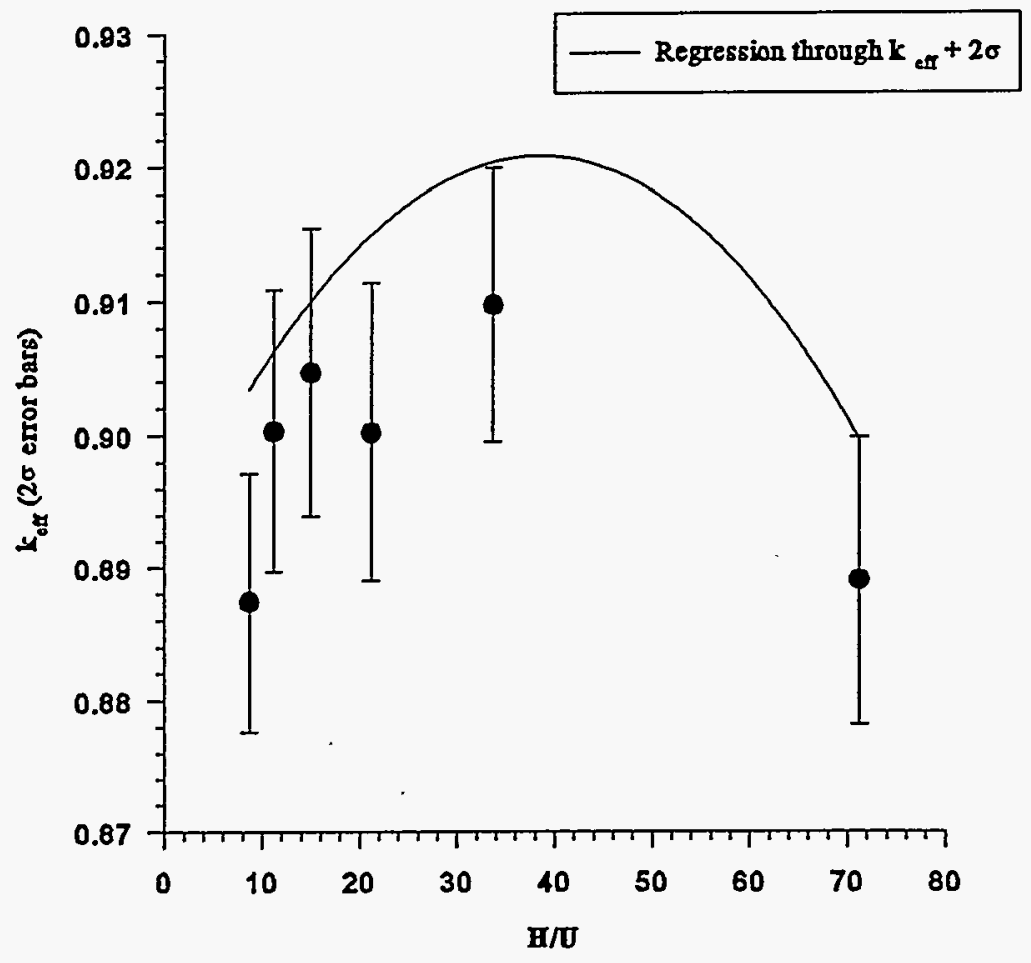

Single $5.25^{\prime \prime}$ by $15^{\prime \prime}$ Container with Full Reflection for $100 \%$ Enriched U308 and water

\begin{tabular}{|c|c|c|c|c|c|}
\hline & & & & & \\
\hline Case & H/U & Keff & K sig & $2^{*} K$ sig & Keff $+2 * K$ sig \\
\hline & & & & & \\
\hline fr-t05.out & 71.2 & 0.889 & 0.0054 & 0.0108 & 0.8998 \\
\hline fr-t10.out & 33.7 & 0.9097 & 0.0051 & 0.0102 & 0.9199 \\
\hline fr-t15.out & 21.2 & 0.9002 & 0.0056 & 0.0112 & 0.9114 \\
\hline fr-t20.out & 15 & 0.9047 & 0.0054 & 0.0108 & 0.9155 \\
\hline fr-t25.out & 11.2 & 0.9003 & 0.0053 & 0.0106 & 0.9109 \\
\hline fr-t30.out & 8.7 & 0.8874 & 0.0049 & 0.0098 & 0.8972 \\
\hline
\end{tabular}


Figure 4.6-6. Single 5.25" ID $\times 15 "$ Tall Cylinder of $\mathrm{U}_{3} \mathrm{O}_{8}$ in a Concrete Corner

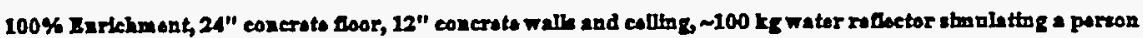

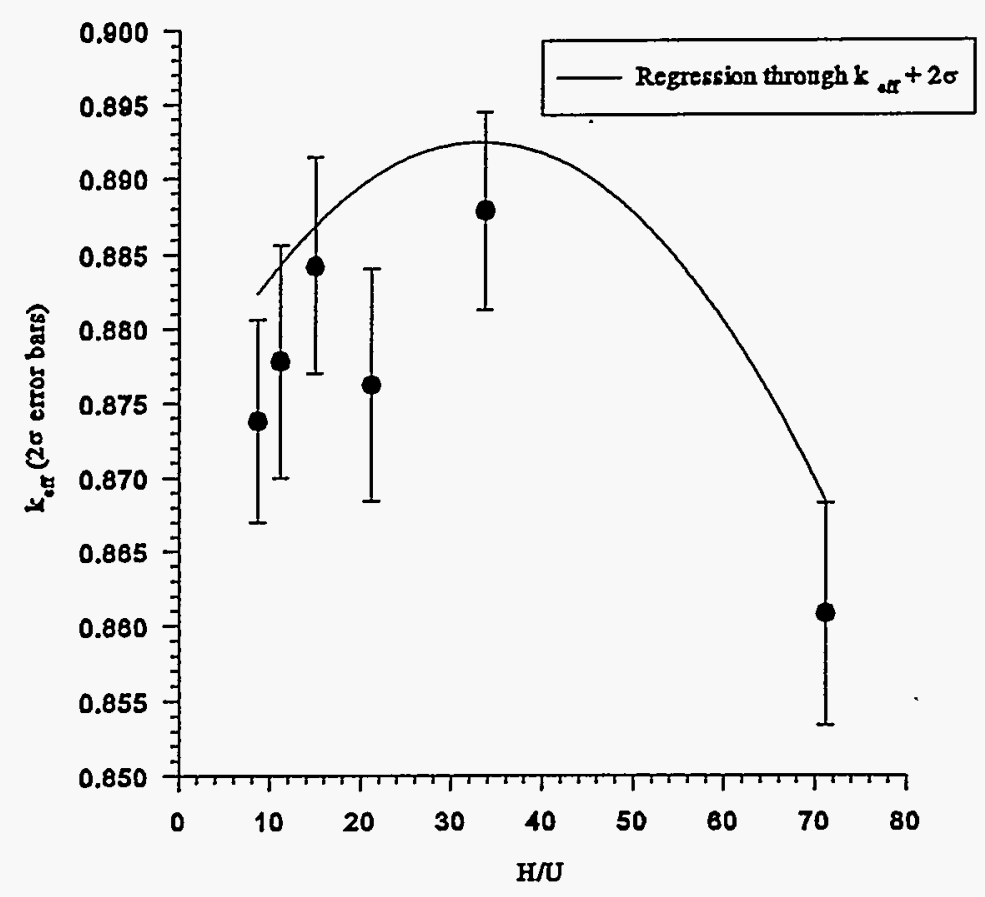

\begin{tabular}{|c|c|c|c|c|c|}
\hline \multicolumn{6}{|c|}{ Single $5.25^{\prime \prime}$ by $15^{\prime \prime}$ Container in a Concrete Corner } \\
\hline \multicolumn{6}{|c|}{ for $100 \%$ Enriched U308 and water } \\
\hline Case & $\mathrm{H} / \mathrm{U}$ & Keff & $\mathrm{K}$ sig & $2^{*} \mathrm{~K}$ sig & Keff $+2^{\star} \mathrm{K}$ sig \\
\hline crnrz05.out & 71.2 & 0.8608 & 0.0037 & 0.0074 & 0.8682 \\
\hline crnrz10.out & 33.7 & 0.8878 & 0.0033 & 0.0066 & 0.8944 \\
\hline crnrz15.out & 21.2 & 0.8762 & 0.0039 & 0.0078 & 0.884 \\
\hline crnr20.out & 15 & 0.8842 & 0.0036 & 0.0072 & 0.8914 \\
\hline crnrz25.out & 11.2 & 0.8778 & 0.0039 & 0.0078 & 0.8856 \\
\hline crnrz30.out & 8.7 & 0.8738 & 0.0034 & 0.0068 & 0.8806 \\
\hline
\end{tabular}


Figure 4.6-7. Two 5.25" ID $\times 15$ "Tall Cylinders of $\mathrm{U}_{3} \mathrm{O}_{8}$ Next to a Wall $100 \%$ Enrichnent, 24" conerete floor, 12" conerete wall and ealing, 100 kg water reflector sionulathg a perzon

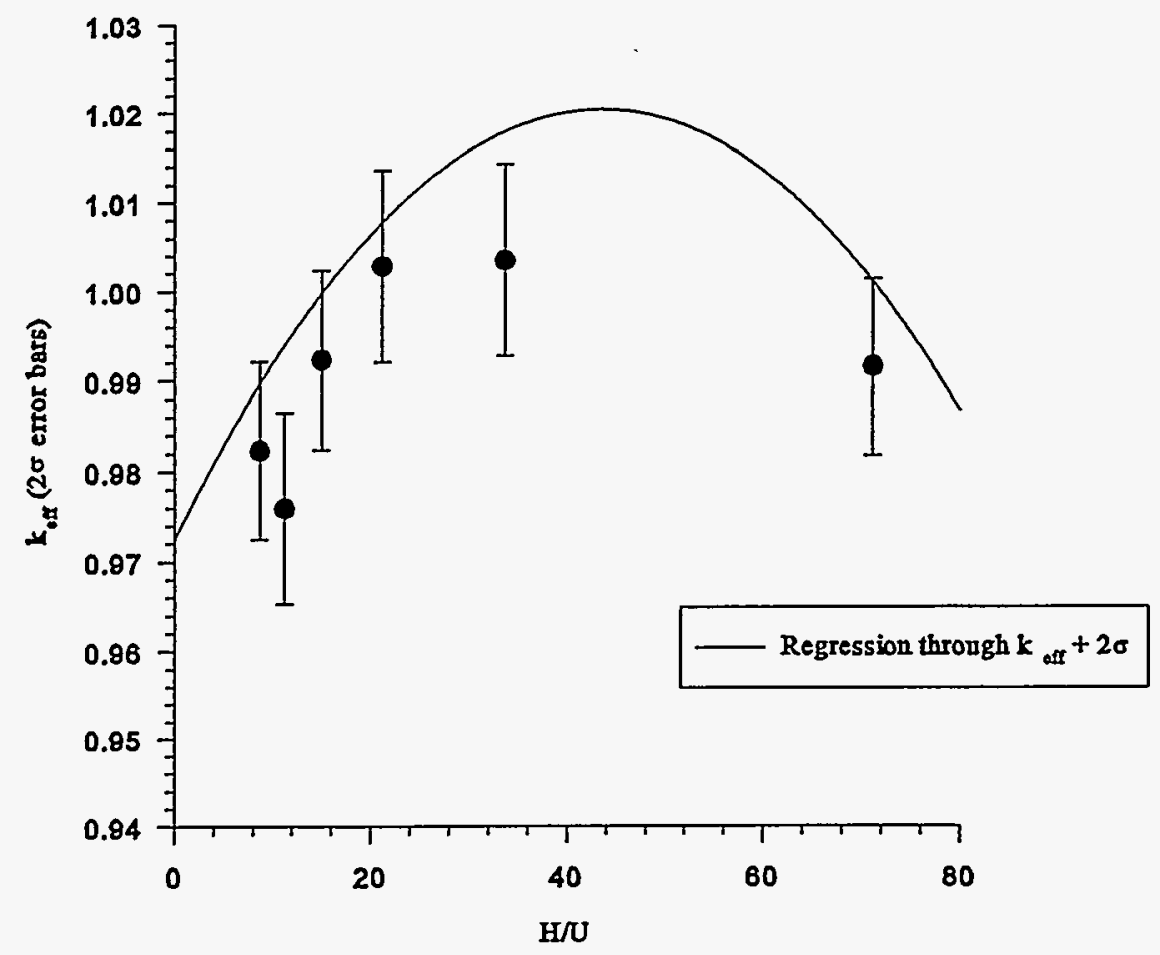

\begin{tabular}{|c|c|c|c|c|c|}
\hline \multicolumn{6}{|c|}{ Two 5.25" by $15^{\prime \prime}$ Tall Containers Adjacent to a Concrete Wall } \\
\hline \multicolumn{6}{|c|}{ for $100 \%$ Enriched U308 and water } \\
\hline & & & & & \\
\hline & & & & & \\
\hline d00nz05.out & 71.2 & 0.9914 & 0.0049 & 0.0098 & 1.0012 \\
\hline doonz10.out & 33.7 & 1.0034 & 0.0054 & 0.0108 & 1.0142 \\
\hline do0nz15.out & 21.2 & 1.0028 & 0.0054 & 0.0108 & 1.0136 \\
\hline do0nz20.out & 15 & 0.9923 & 0.005 & 0.01 & 1.0023 \\
\hline do0nz25.out & 11.2 & 0.9759 & 0.0053 & 0.0106 & 0.9865 \\
\hline doonz30.out & 8.7 & 0.9823 & 0.0049 & 0.0098 & 0.9921 \\
\hline
\end{tabular}




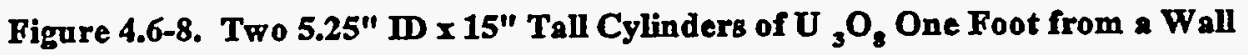

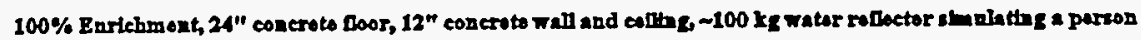

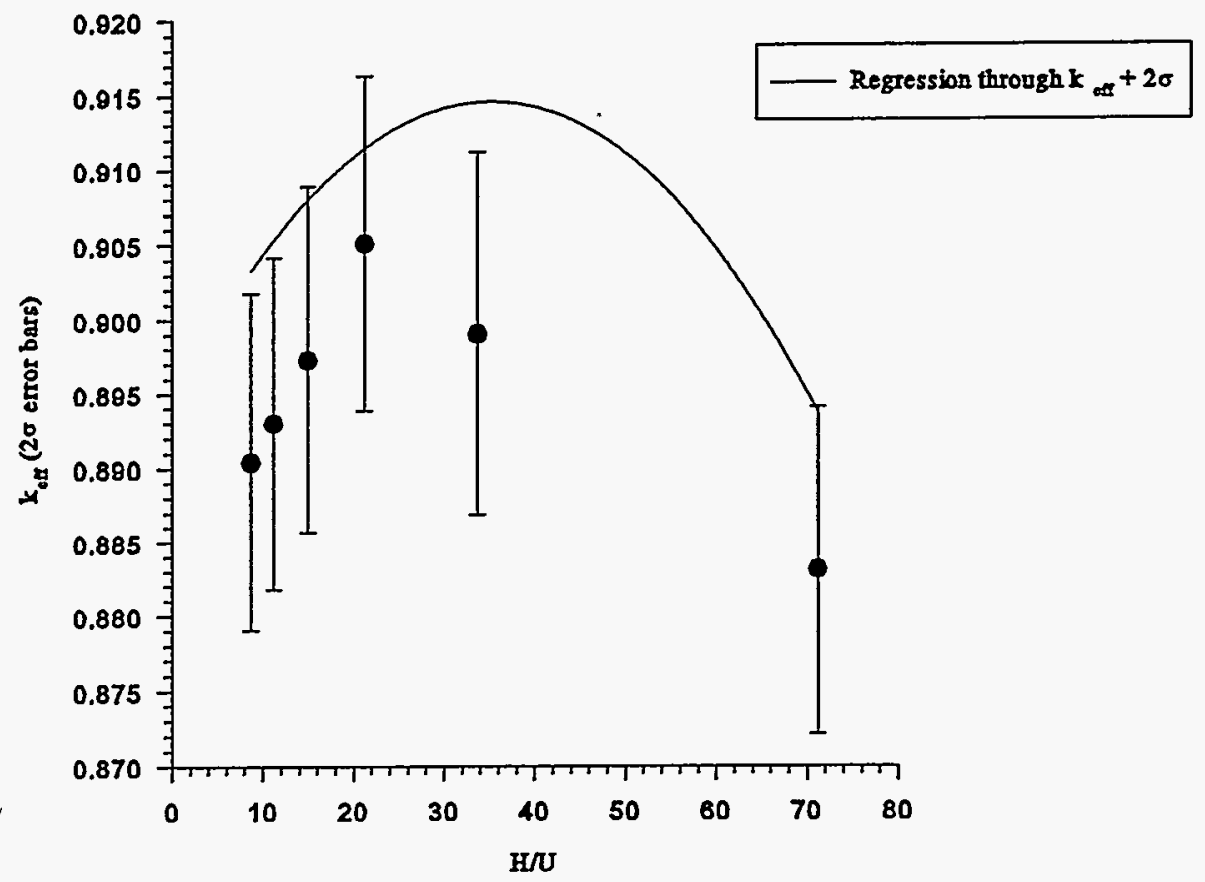

Two $5.25^{\prime \prime}$ by $15^{\prime \prime}$ Tall Containers One Foot from a Concrete Wall for $100 \%$ Enriched U3O8 and water

\begin{tabular}{|c|c|c|c|c|c|}
\hline & & & & & \\
\hline Case & HN & Keff & K sig & $2^{\star} K$ sig & Keff $+2^{\star} K$ sig \\
\hline & & & & & \\
\hline$d 12 n-c z .05$ & 71.2 & 0.8831 & 0.00549 & 0.01098 & 0.89408 \\
\hline$d 12 n-c z .10$ & 33.7 & 0.899 & 0.00607 & 0.01214 & 0.91114 \\
\hline$d 12 n-c z .15$ & 21.2 & 0.9051 & 0.00561 & 0.01122 & 0.91632 \\
\hline$d 12 n-c z .20$ & 15 & 0.8973 & 0.00579 & 0.01158 & 0.90888 \\
\hline$d 12 n-c z .25$ & 11.2 & 0.893 & 0.0056 & 0.0112 & 0.9042 \\
\hline$d 12 n-c z .30$ & 8.7 & 0.8904 & 0.00569 & 0.01138 & 0.90178 \\
\hline
\end{tabular}


Figure 4.6-9. An Array of 5.25" $\mathrm{DD} \times 15$ " Tall Cylinders of $\mathrm{U}_{3} \mathrm{O}_{8}$

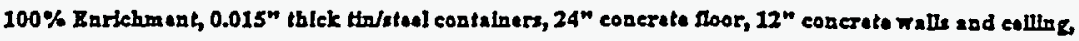
6' upxelag betreen rot zotz, 23" spactag between contalners, 1' upacing trom walle

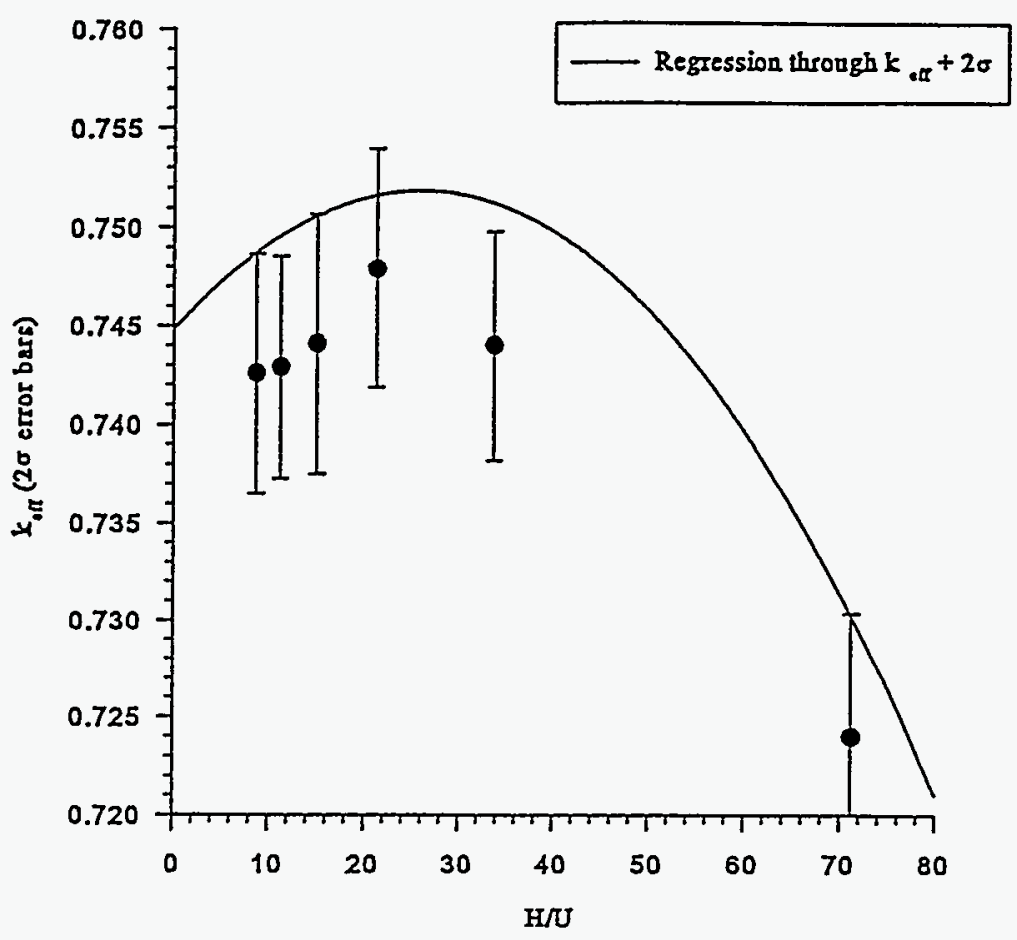

\begin{tabular}{|c|c|c|c|c|c|}
\hline \multicolumn{7}{|l|}{ An Array of $5.25^{\mathrm{m}} \times 15^{\mathrm{m}} \mathrm{Tin} / \mathrm{Steel}$ Containers of U308 } \\
\hline For $100 \%$ Enrichment & & & & & \\
\hline & & & & & \\
\hline Case & HIU & Keff & $\mathrm{K} \mathrm{sig}$ & $2^{*} \mathrm{~K} \mathrm{sig}$ & Keff+2*K sig \\
\hline & & & & & \\
\hline array-z.05.0 & 71.2 & 0.724 & 0.00318 & 0.00636 & 0.73036 \\
\hline array-z.10.0 & 33.7 & 0.744 & 0.0029 & 0.0058 & 0.7498 \\
\hline array-z.15.0 & 21.2 & 0.7479 & 0.00302 & 0.00604 & 0.75394 \\
\hline array-z.20.0 & 15 & 0.7441 & 0.003295 & 0.00659 & 0.75069 \\
\hline array-z.25.0 & 11.2 & 0.7429 & 0.00282 & 0.00564 & 0.74854 \\
\hline array-z.30.0 & 8.7 & 0.7426 & 0.00303 & 0.00606 & 0.74866 \\
\hline
\end{tabular}


Figure 4.6-10. The Effects of Interstitial Water Volume Fraction on $k$,ff

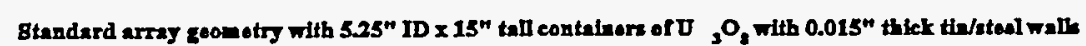

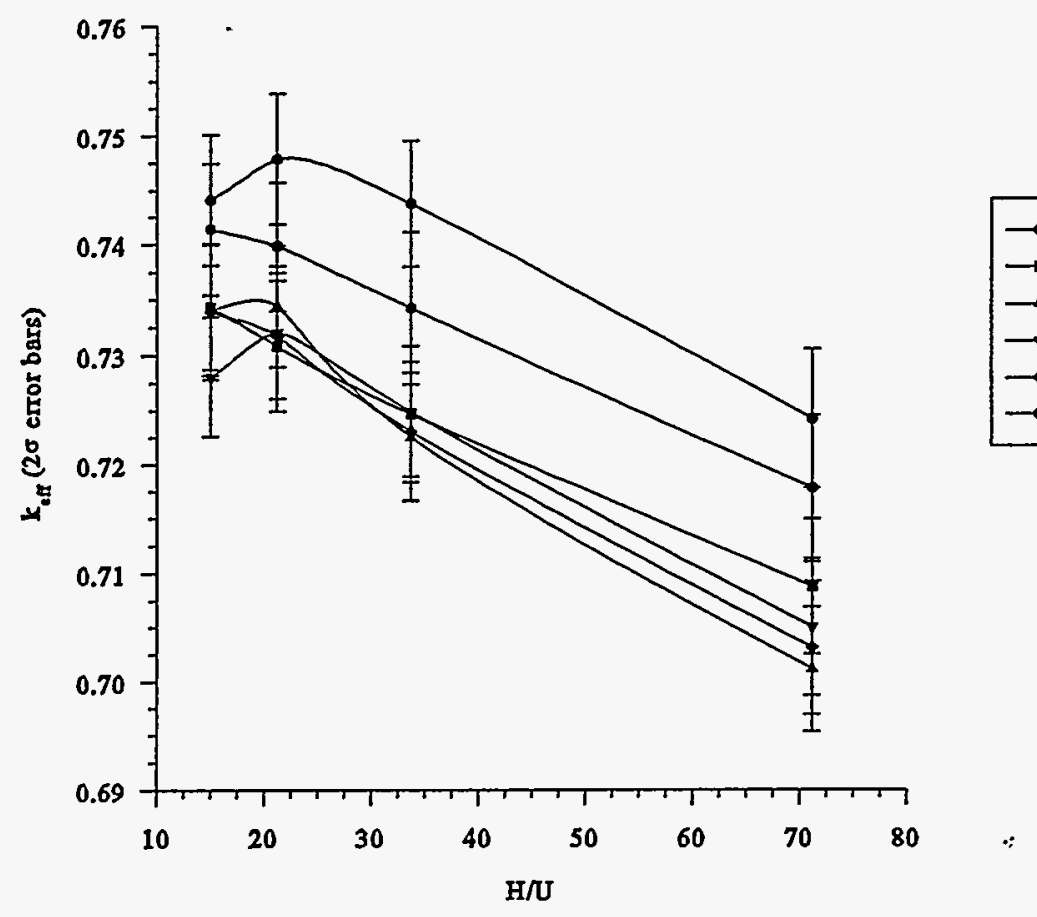


Figure 4.6-10a. The Effects of Interstitial Water Volume Fraction on $\mathbf{k}_{\text {eff }}$ Standard array geometry with 5.25" ID $\times 15$ " tall containers of $\mathrm{U}_{3} \mathrm{O}_{8}$ with $0.015^{\prime \prime}$ thick tin/steel walls

\begin{tabular}{|c|c|c|c|c|c|}
\hline \multicolumn{6}{|c|}{ The Effects of Interstitial Water Volume Fraction for a Standard } \\
\hline \multicolumn{6}{|c|}{ Array of 5.25" ID $\times 15^{\prime \prime}$ Tin/Steel Containers of U3O8 } \\
\hline Case & $\mathrm{H} / \mathrm{U}$ & Keff & K_sig & $2 * \mathrm{~K}$ sig & Keff $+2^{*} K$ sig \\
\hline Interstitial & Water $=$ & 0 & & & \\
\hline array-z.05.o & 71.2 & 0.7241 & 0.0032 & 0.0064 & 0.7305 \\
\hline array-z.10.0 & 33.7 & 0.7437 & 0.0029 & 0.0058 & 0.7495 \\
\hline array-z.15.0 & 21.2 & 0.7478 & 0.003 & 0.006 & 0.7538 \\
\hline array-z.20.0 & 15 & 0.7441 & 0.003 & 0.006 & 0.7501 \\
\hline Interstitial & Water $=$ & 0.001 & & & \\
\hline $\operatorname{arr} \sim z \sim w 1.05 .0$ & 71.2 & 0.7087 & 0.00308 & 0.00616 & 0.71486 \\
\hline $\operatorname{arr} \sim z \sim w 1.10 .0$ & 33.7 & 0.7246 & 0.00314 & 0.00628 & 0.73088 \\
\hline $\operatorname{arr} \sim z \sim w 1.15 .0$ & 21.2 & 0.7308 & 0.00295 & 0.0059 & 0.7367 \\
\hline $\operatorname{arr} \sim z \sim w 1.20 .0$ & 15 & 0.7343 & 0.0028 & 0.0056 & 0.7399 \\
\hline Interstitial & Water $=$ & 0.005 & & & \\
\hline arr Z W2.05.0 & 71.2 & 0.7011 & 0.00288 & 0.00576 & 0.70686 \\
\hline $\operatorname{arr} \sim z \sim w 2.10 .0$ & 33.7 & 0.7225 & 0.00296 & 0.00592 & 0.72842 \\
\hline $\operatorname{arr} \sim z \sim W 2.15 .0$ & 21.2 & 0.7344 & 0.00274 & 0.00548 & 0.73988 \\
\hline $\operatorname{arr} \sim Z \sim W 2.20 .0$ & 15 & 0.7341 & 0.00296 & 0.00592 & 0.74002 \\
\hline Interstitial & Water $=$ & 0.01 & & & \\
\hline $\operatorname{arr} \sim z \sim w 3.05 .0$ & 71.2 & 0.705 & 0.00315 & 0.0063 & 0.7113 \\
\hline $\operatorname{arr} \sim z \sim w 3.10 .0$ & 33.7 & 0.7248 & 0.00298 & 0.00596 & 0.73076 \\
\hline $\operatorname{arr} \sim z \sim w 3.15 .0$ & 21.2 & 0.732 & 0.003 & 0.006 & 0.738 \\
\hline $\operatorname{arr} \sim z \sim w 3.20 .0$ & 15 & 0.728 & 0.00272 & 0.00544 & 0.73344 \\
\hline Interstitial & Water $=$ & 0.05 & & & \\
\hline arr Z $\sim W 4.05 .0$ & 71.2 & 0.7031 & 0.00307 & 0.00614 & 0.70924 \\
\hline arr Z $\sim w 4.10 .0$ & 33.7 & 0.723 & 0.0032 & 0.0064 & 0.7294 \\
\hline $\operatorname{arr} \sim z \sim w 4.15 .0$ & 21.2 & 0.7317 & 0.00284 & 0.00568 & 0.73738 \\
\hline arr $\sim z \sim w 4.20 .0$ & 15 & 0.7339 & 0.00307 & 0.00614 & 0.74004 \\
\hline Interstitial & Water $=$ & 0.1 & & & \\
\hline arr Z Z w5.05.0 & 71.2 & 0.7177 & 0.00336 & 0.00672 & 0.72442 \\
\hline arr Z w5.10.0 & 33.7 & 0.7342 & 0.00343 & 0.00686 & 0.74106 \\
\hline arr z w5.15.o & 21.2 & 0.7398 & 0.00293 & 0.00586 & 0.74566 \\
\hline $\operatorname{arr} \sim z \sim w 5.20 .0$ & 15 & 0.7414 & 0.003 & 0.006 & 0.7474 \\
\hline
\end{tabular}


Figure 4.6.11 - An Array of 5.25" $\mathrm{D} \times 15^{\prime \prime}$ Tall Cylinders of $U_{3} O_{8}$

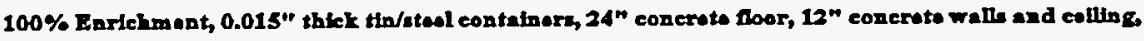
6' spacing between row zots, 23" speeing between contaluers, 1' spaclng trom walls

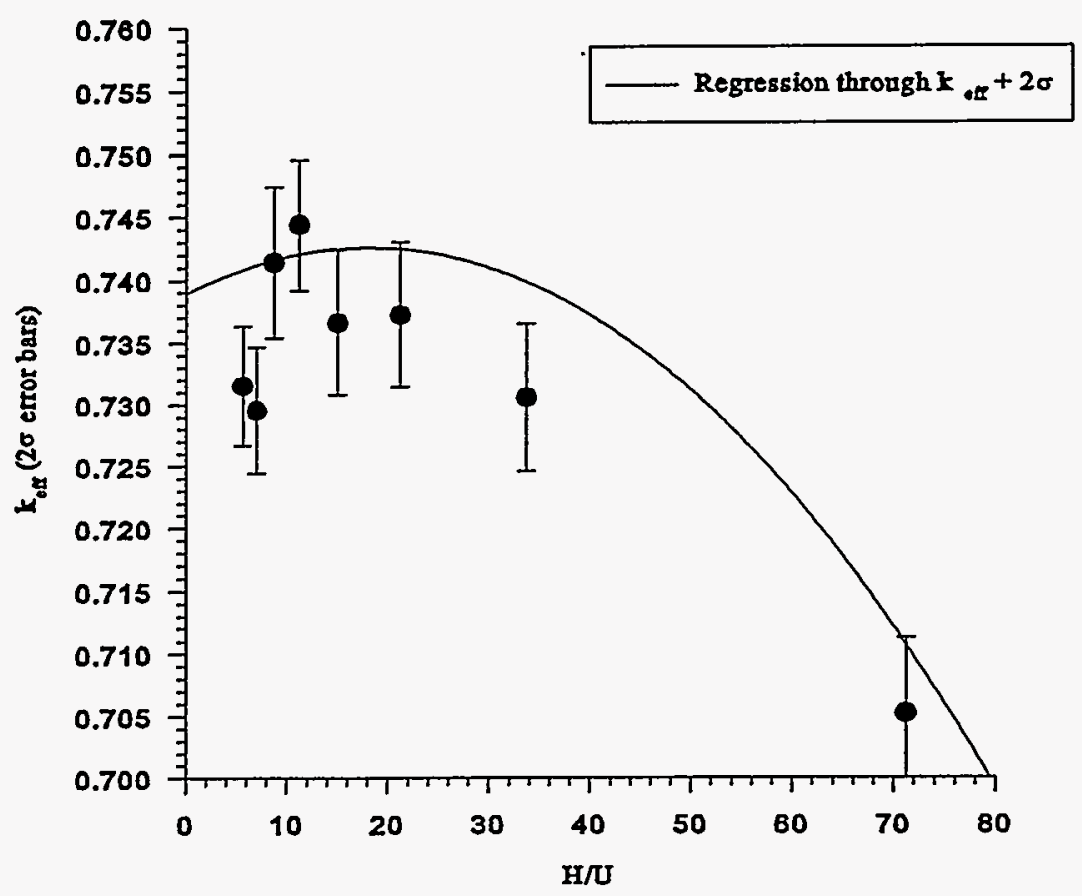

An Array of $5.25^{\prime \prime} \times 15^{\prime \prime}$ Tin/Steel Containers of U308 For $100 \%$ Enrichment, Overloaded Storage Position

\begin{tabular}{|c|c|c|c|c|c|}
\hline & & & & & \\
\hline Case & HN & Keff & K sig & $2^{\star} \mathrm{K}$ sig & Keff $+2^{\star} \mathrm{K}$ sig \\
\hline & & & & & \\
\hline arrzcon.05.0 & 71.2 & 0.7052 & 0.003 & 0.006 & 0.7112 \\
\hline arrzcon.10.0 & 33.7 & 0.7305 & 0.003 & 0.006 & 0.7365 \\
\hline arrzcon.15.0 & 21.2 & 0.7372 & 0.0029 & 0.0058 & 0.743 \\
\hline arrzcon.20.0 & 15 & 0.7366 & 0.0029 & 0.0058 & 0.7424 \\
\hline arrzcon.25.0 & 11.2 & 0.7444 & 0.0026 & 0.0052 & 0.7496 \\
\hline arrzcon.30.0 & 8.7 & 0.7414 & 0.003 & 0.006 & 0.7474 \\
\hline arrzcon.35.0 & 6.96 & 0.7295 & 0.00257 & 0.00514 & 0.73464 \\
\hline arrzcon.40.0 & 5.62 & 0.7315 & 0.00242 & 0.00484 & 0.73634 \\
\hline
\end{tabular}


POEF-SH-30

\subsection{0-inch by 9.0-inch Polybottle (CO-4)}

This section presents results of calculations designed to model the $\mathrm{CO}-4$ polybottles described in Table 1.0-1. Table 4.7-0 gives the figure and table numbers that contain the geometry models and fuel materials. Standard array cases are bounded by the analysis performed for the more reactive material $\mathrm{UO}_{2} \mathrm{~F}_{2}$ in 5.0 -inch by 24 -inch polybottles. The reactivity of $\mathrm{U}_{3} \mathrm{O}_{8}$ and $\mathrm{UO} 2 \mathrm{~F} 2$ is not significantly different as shown by a comparison of Figures 4.7-5 and 4.4.1-1. Note, the $\mathrm{U}_{3} \mathrm{O}_{8}$ cases presented in this section do not include the polybottle container in the model. However, the additional reactivity that is expected is not significant as discussed in Section 4.1.2; therefore, either material could be used for this container analysis. The results of this study validate bounding the 5.0 -inch by 9.0 -inch array case using the $\mathrm{UO}_{2} \mathrm{~F}_{2}$ results. The geometry model designators are explained in Table 4.1-1.

Table 4.7-0. Summary of References for CO-4 Polybottles

\begin{tabular}{|c|c|c|}
\hline Geometry Model & Material & Figure/Table Number \\
\hline \hline SFR & $\mathrm{U}_{3} \mathrm{O}_{8}$ & $4.7-1$ \\
\hline SCC & $\mathrm{U}_{3} \mathrm{O}_{8}$ & $4.7-2$ \\
\hline TACW & $\mathrm{U}_{3} \mathrm{O}_{8}$ & $4.7-3$ \\
\hline TSCW & $\mathrm{U}_{3} \mathrm{O}_{8}$ & $4.7-4$ \\
\hline
\end{tabular}


Figare 4.7-1. Single Fully Reflected 5.00" ID $\times 9$ " Poly. Container of $\mathrm{U}_{3} \mathrm{O}_{8}$

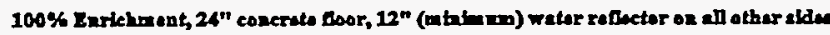

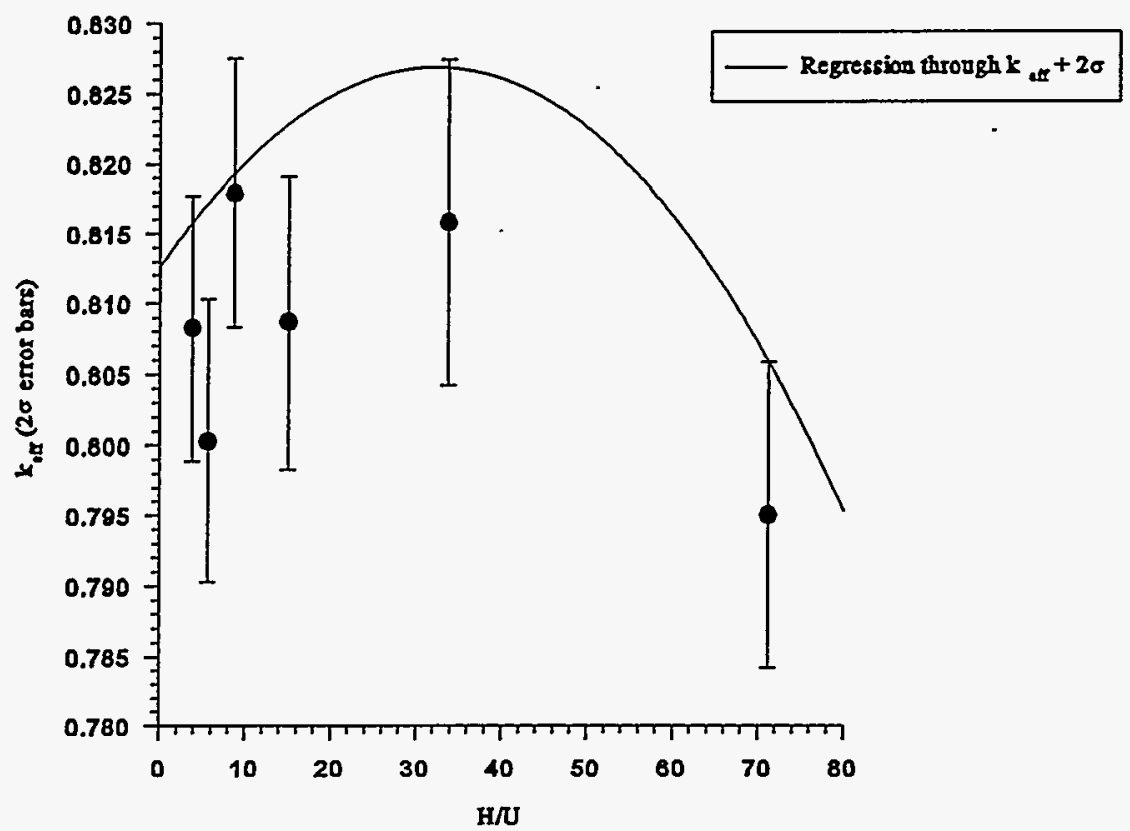

Single Fully Relected 5.0 " by 9 " Poly. Container for $100 \%$ Enriched U308 and water

\begin{tabular}{|c|c|c|c|c|c|}
\hline & & & & & \\
\hline Case & HIU & Keff & K_sig & $2^{\star} K$ Ksig & Keff+2*K_sig \\
\hline & & & & & \\
\hline frco405.out & 71.2 & 0.795 & 0.0054 & 0.0108 & 0.8058 \\
\hline frco41.out & 33.75 & 0.8158 & 0.0058 & 0.0116 & 0.8274 \\
\hline frco42.out & 15 & 0.8087 & 0.0052 & 0.0104 & 0.8191 \\
\hline frco43.out & 8.7 & 0.8179 & 0.0048 & 0.0096 & 0.8275 \\
\hline frco44.out & 5.62 & 0.8003 & 0.005 & 0.01 & 0.8103 \\
\hline frco45.out & 3.8 & 0.8083 & 0.0047 & 0.0094 & 0.8177 \\
\hline
\end{tabular}


Figure 4.7-2. Single 5.00" ID $\times$ 9" Tall Cylinder of $\mathrm{U}_{3} \mathrm{O}_{8}$ in a Concrete Corner

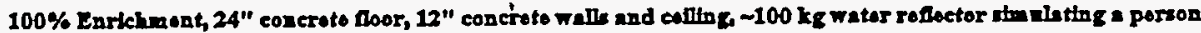

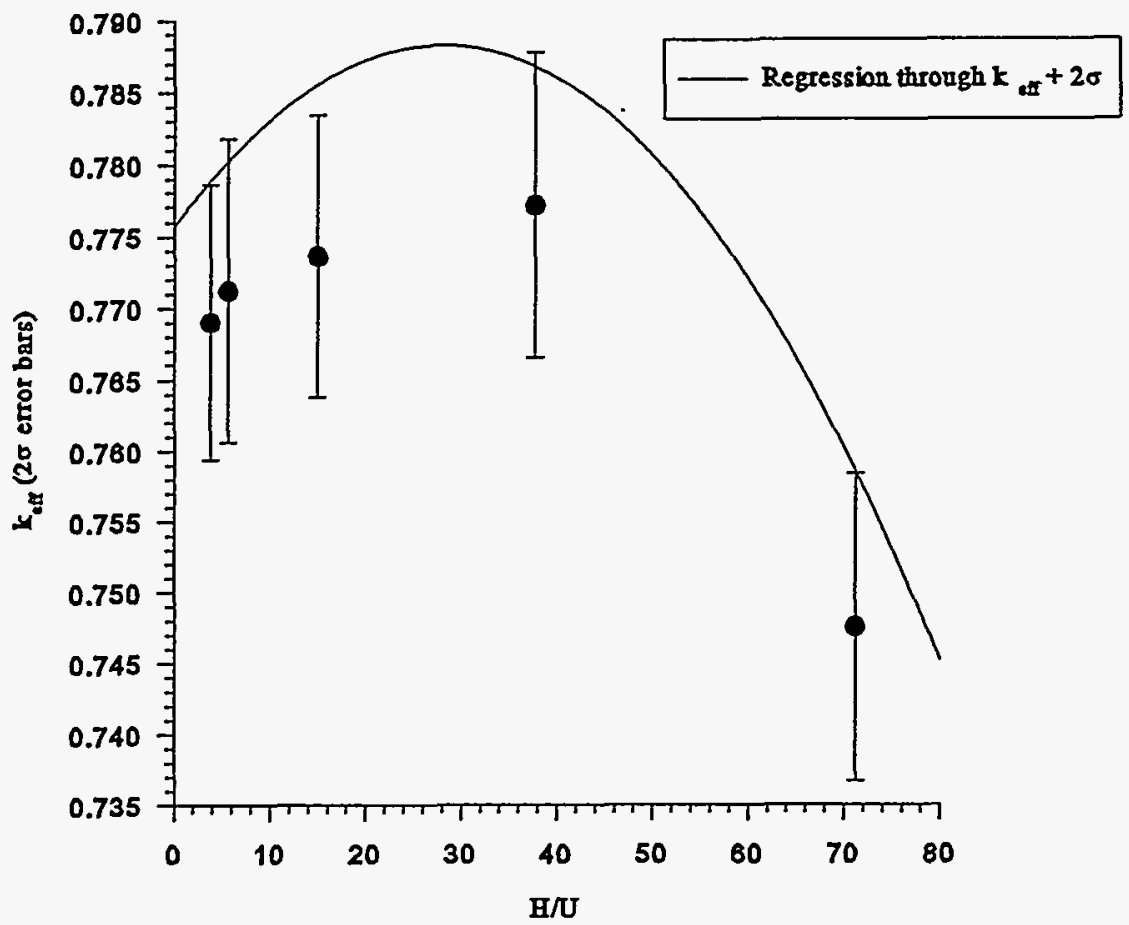

Single 5.0 in. Poly. Container in a Concrete Corner for $100 \%$ Enriched U308 and water

\begin{tabular}{|c|c|c|c|c|c|}
\hline & & & & & \\
\hline Case & HN & Keff & K sig & $2^{*} \mathrm{~K}$ sig & Keff $+2^{*} \mathrm{~K}$ sig \\
\hline & & & & & \\
\hline crnco405.out & 71.2 & 0.7475 & 0.0054 & 0.0108 & 0.7583 \\
\hline crnco41.out & 33.75 & 0.7771 & 0.0053 & 0.0106 & 0.7877 \\
\hline crnco42.out & 15 & 0.7736 & 0.0049 & 0.0098 & 0.7834 \\
\hline crnco43.out & 8.7 & 0.7712 & 0.0053 & 0.0106 & 0.7818 \\
\hline crnco44.out & 5.62 & 0.769 & 0.0048 & 0.0096 & 0.7786 \\
\hline
\end{tabular}


POEF-SH-30

Figure 4.7-3. Two 5.00" ID $~ 9$ " Tall Cylinders of $\mathrm{U}_{3} \mathrm{O}_{8}$ Nert to a Wall

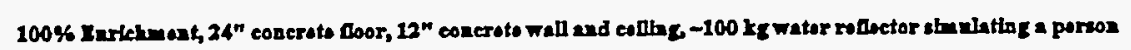

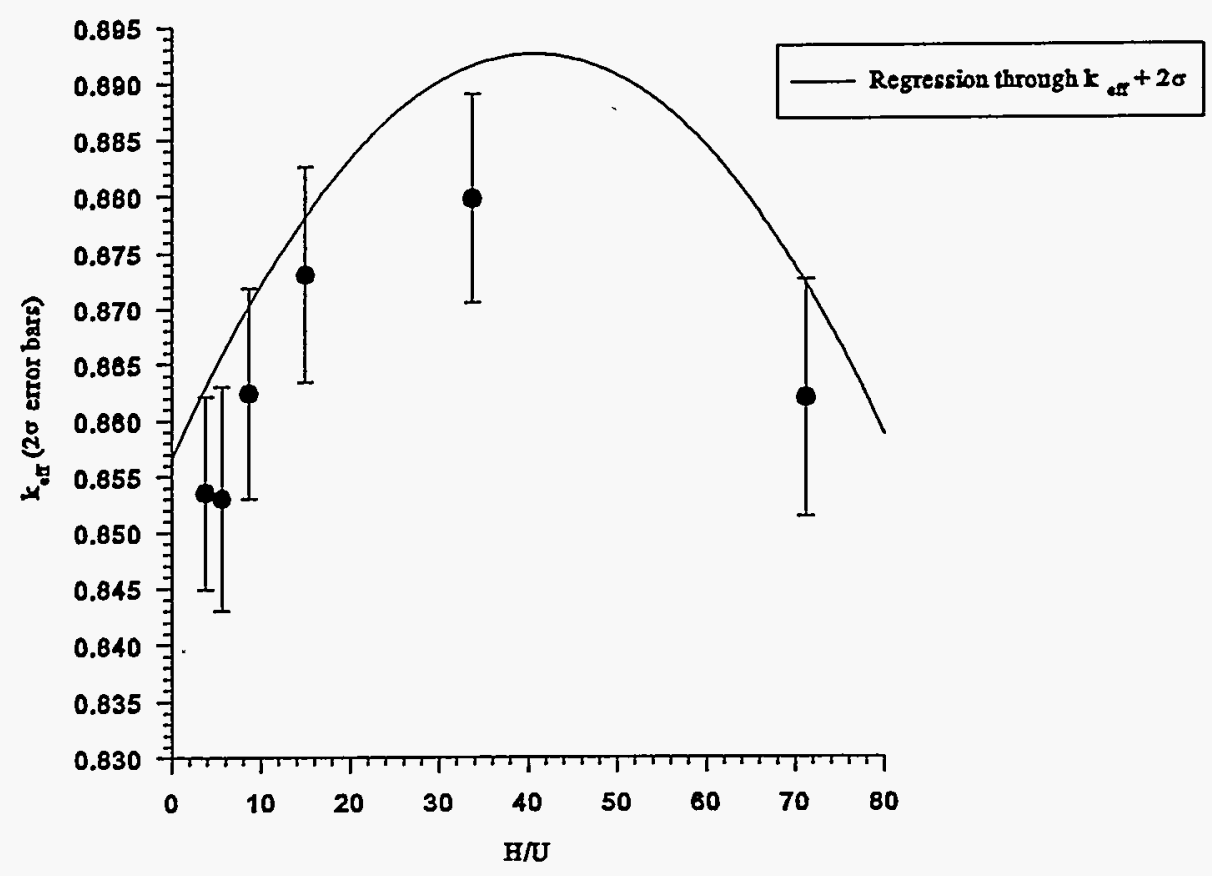

Two 5.0 in. Poly. Containers Adjacent to a Wall for $100 \%$ Enriched U308 and water

\begin{tabular}{|c|c|c|c|c|c|}
\hline & & & & & \\
\hline Case & H/U & Keff & K_sig & $2^{*}$ K_sig & Keff+2*K_sig \\
\hline & & & & & \\
\hline d00n05.out & 71.2 & 0.8619 & 0.0053 & 0.0106 & 0.8725 \\
\hline d00n1.out & 33.75 & 0.8797 & 0.0046 & 0.0092 & 0.8889 \\
\hline d00n2.out & 15 & 0.873 & 0.0048 & 0.0096 & 0.8826 \\
\hline d00n3.out & 8.7 & 0.8624 & 0.0047 & 0.0094 & 0.8718 \\
\hline d00n4.out & 5.62 & 0.853 & 0.005 & 0.01 & 0.863 \\
\hline d00n5.out & 3.8 & 0.8535 & 0.0043 & 0.0086 & 0.8621 \\
\hline
\end{tabular}


Figure 4.7-4. Two 5.00" $\mathrm{D} \times 9$ " Tall Cylinders of $\mathrm{U}_{3} \mathrm{O}_{8}$ One Foot from a Wall

100\% Enrichnent, 24" concroto floor, 12" concrete wall and colling, $-100 \mathrm{~kg}$ water roflector sianglatiog a poreon

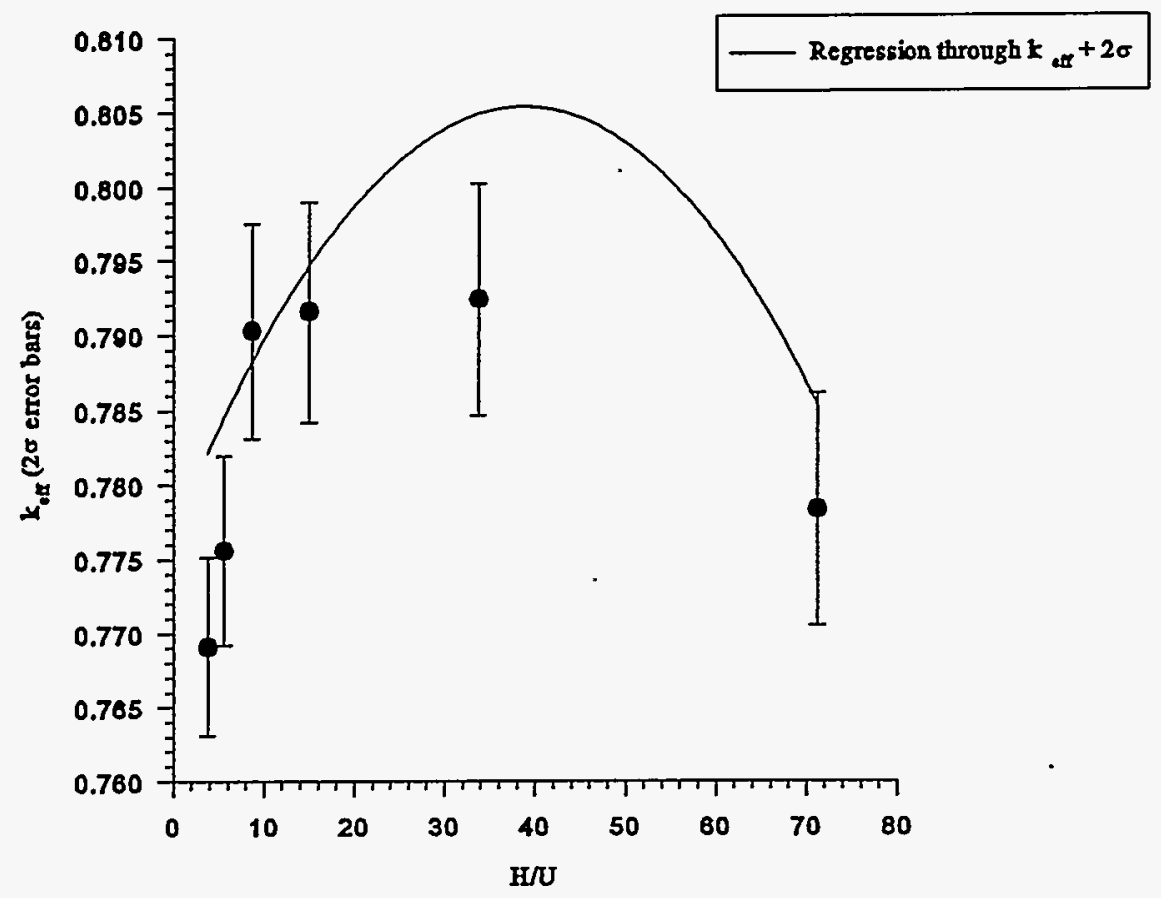

\begin{tabular}{|c|c|c|c|c|c|}
\hline \multicolumn{6}{|c|}{ Two 5.0 in. Poly. Containers $1^{\prime}$ from Concrete Wall } \\
\hline \multicolumn{6}{|c|}{ for $100 \%$ Enriched U308 and water } \\
\hline & & & & & \\
\hline Case & $\mathrm{H} / \mathrm{U}$ & Keff & K_sig & $2^{\star} \mathrm{K}$ sig & Keff $+2^{*} \mathrm{~K}$ sig \\
\hline d2nco405.out & 71.2 & 0.7783 & 0.0039 & 0.0078 & 0.7861 \\
\hline d2nco41.out & 33.7 & 0.7924 & 0.0039 & 0.0078 & 0.8002 \\
\hline d2nco42.out & 21.2 & 0.7916 & 0.0037 & 0.0074 & 0.799 \\
\hline d2nco43.out & 15 & 0.7903 & 0.0036 & 0.0072 & 0.7975 \\
\hline d2nco44.out & 11.2 & 0.7756 & 0.0032 & 0.0064 & 0.782 \\
\hline d2nco45.out & 8.1 & 0.7691 & 0.003 & 0.006 & 0.7751 \\
\hline
\end{tabular}


Figure 4.7-5. Single Fully Reflected 5.00" I $\times 24^{\prime \prime}$ Tall Cylinder of $\mathrm{U}_{3} \mathrm{O}_{8}$

100\% Enrichment, 24" conereto Hoor, 12" (minbmum) wator roflactor on all other aldes

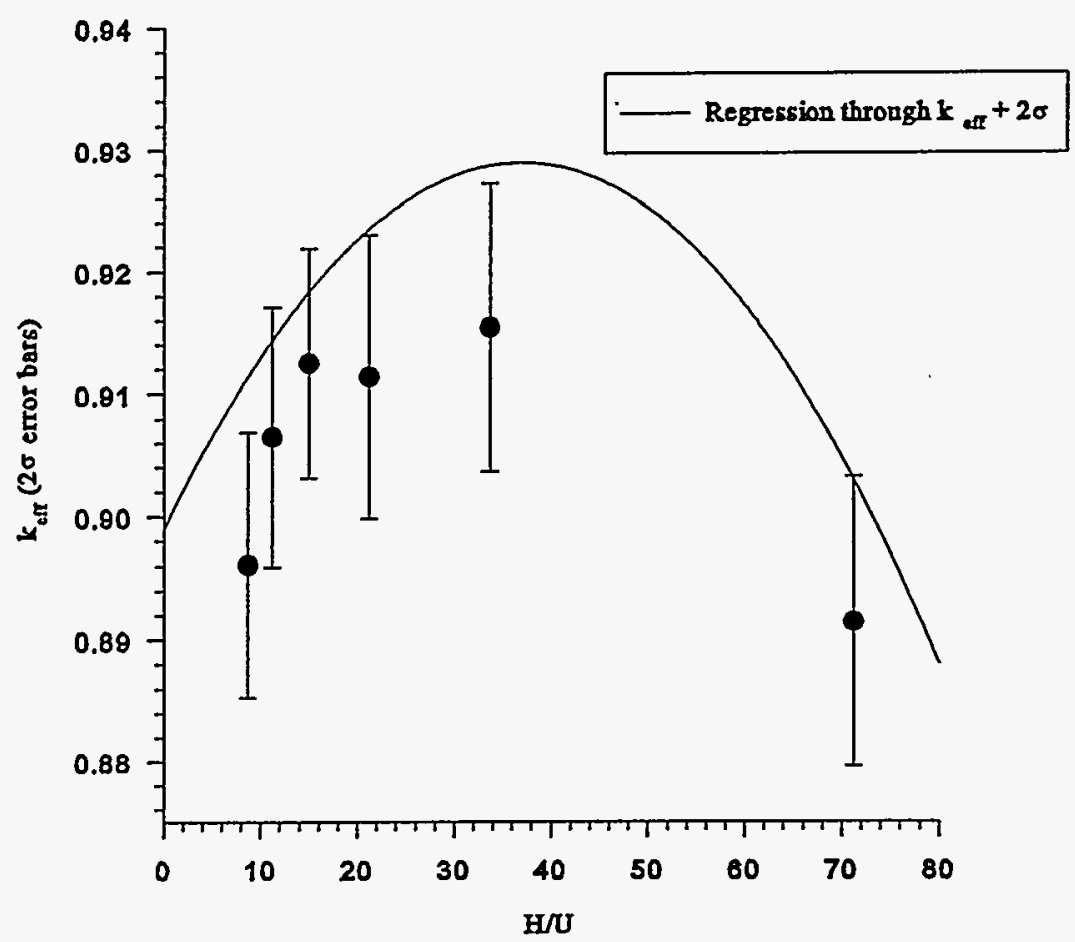

Single Fully Relected 5.0 " by 24 " Poly. Container for $100 \%$ Enriched U3O8 and Water

\begin{tabular}{|c|c|c|c|c|c|}
\hline & & & & & \\
\hline Case & H/U & Keff & K_sig & $2^{*}$ K_sig & Keff $+2^{*}$ K_sig \\
\hline & & & & & \\
\hline fr24-05.out & 71.2 & 0.8914 & 0.0059 & 0.0118 & 0.9032 \\
\hline fr24-10.out & 33.7 & 0.9154 & 0.0059 & 0.0118 & 0.9272 \\
\hline fr24-15.out & 21.2 & 0.9114 & 0.0058 & 0.0116 & 0.923 \\
\hline fr24-20.out & 15 & 0.9125 & 0.0047 & 0.0094 & 0.9219 \\
\hline fr24-25.out & 11.2 & 0.9065 & 0.0053 & 0.0106 & 0.9171 \\
\hline fr24-30.out & 8.7 & 0.8961 & 0.0054 & 0.0108 & 0.9069 \\
\hline
\end{tabular}


POEF-SH-30

\subsection{Supplemental $\mathrm{UF}_{4}+$ Oil Array Cases}

The most reactive array of those performed for the $100 \%$ enriched cases are 5.00 " ID $\times 24$ " tall polyethylene containers filled with $\mathrm{UF}_{4}+$ oil (Figure 4.4.1-10). The position of this array was moved to 1-foot from all surrounding reflecting concrete walls. The results are shown in Figure 4.8-5. Based on this result, all arrays considered in this analysis fall within the safety criteria when moved to within 1-foot from surrounding concrete walls.

The 1-foot spacing limit is not required for $10 \%$ enriched material based on additional array analysis presented herein using no separation from the concrete walls. The study uses $\mathrm{UF}_{4}$ toil which was shown previously to be more reactive than $\mathrm{UO}_{2} \mathrm{~F}_{2}+\mathrm{H}_{2} \mathrm{O}$ and $\mathrm{U}_{3} \mathrm{O}_{8}$; therefore, $\mathrm{UF}_{4}$ toil was used as the bounding material for this analysis. Each of the container sizes was evaluated using the standard array model with no separation from the surrounding concrete walls to show the effects of surrounding the array with a close fitting concrete enclosure.

Figure 4.8-1 4.32" ID x 50.4" standard array with no separation from concrete walls (UFF ${ }_{4}$ +oil).

Figure 4.8-2 5.0" $\mathrm{ID}$ x 24.0" standard array with no separation from concrete walls $\left(\mathrm{UF}_{4}+\right.$ oil).

Figure 4.8-3 5.25" $\mathrm{ID} \times 24.0^{\prime \prime}$ standard array with no separation for concrete walls $\left(\mathrm{UF}_{4}+\mathrm{oil}\right)$.

The most reactive of the contingency cases is overloading a storage position, as shown by comparing Figures 4.3.1-10, 4.3.1-12, and 4.3.1-14. The most reactive container in an array (5.25" ID x 24" tall containers) was shown to meet the safety criteria by modeling two containers side by side with full water reflection. The results are shown in Figure 4.8-4. For less than $10 \%$ enriched materials, polyethylene was used as the container material for all of the can types.

An additional set of cases was run for an overloaded storage position in an array with 5.25" 
POEF-SH-30

$\mathrm{ID} \times 24^{\prime \prime}$ containers, $10 \%$ enriched $\mathrm{UF}_{4}+\mathrm{oil}$, assuming the containers are $0.195 "$ polyethylene, and next to a 12 " thick concrete wall on all sides of the array. The results are shown in Figure 4.8-6. 
POEF-SH-30

Figure 4.8-1 An Array of 4.32" ID I 50" Tall Cyllnders of UP 4 toll

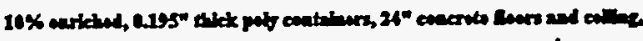

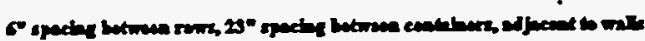

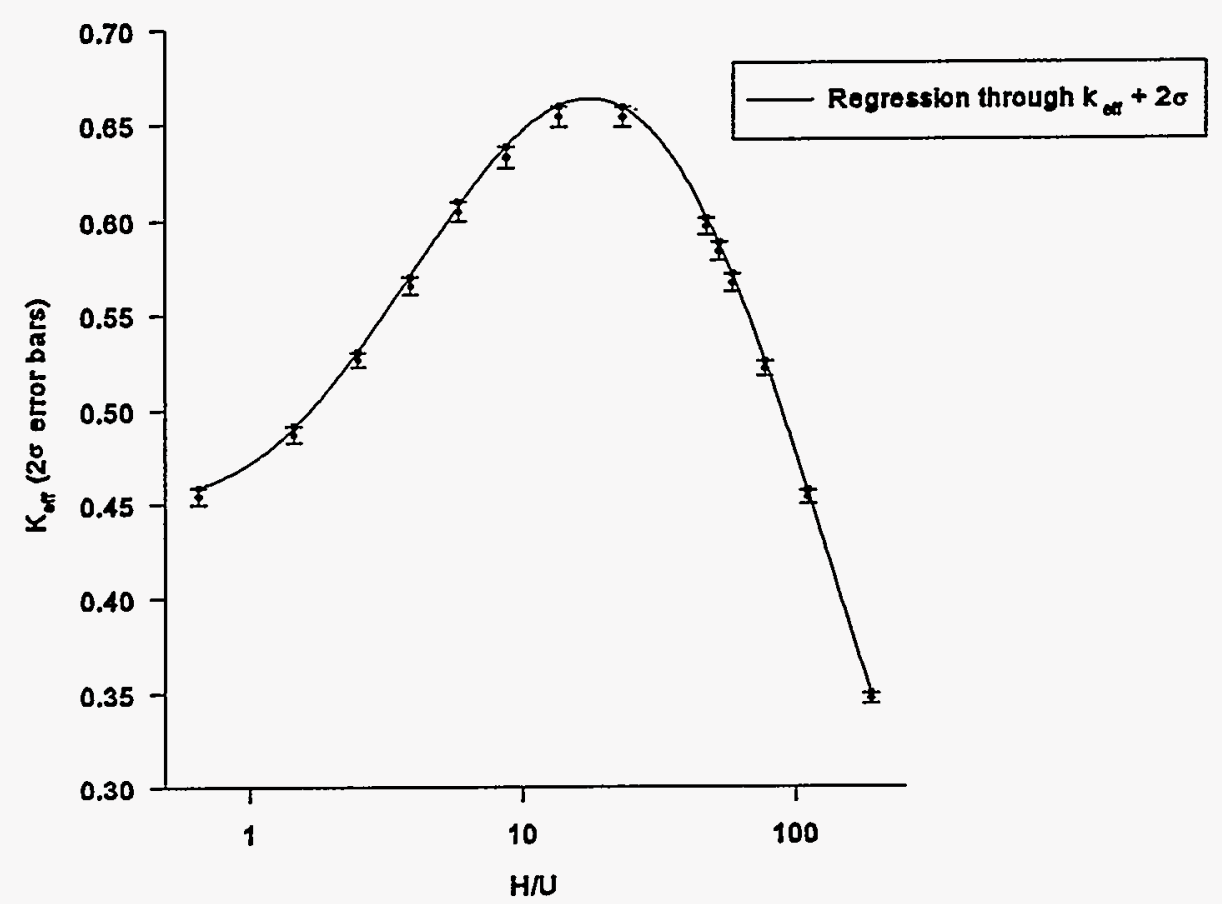

An Array of 4.32" $\times 50^{\prime \prime}$ Cylinders of UF4+oil Next to a Wall $10 \%$ Enrichment, 0.195 " thick Poly Container

\begin{tabular}{|c|c|c|c|c|c|}
\hline Case & HN & Keff & K sig & $2^{\star}$ K sig & Keff $+2^{\star}$ K sig \\
\hline \hline arrdwall.p65.0 & 0.65 & 0.45435 & 0.00218 & 0.00436 & 0.45871 \\
\hline arrdwall.145.o & 1.45 & 0.48723 & 0.00217 & 0.00434 & 0.49157 \\
\hline arrdwall.249.0 & 2.49 & 0.52622 & 0.00198 & 0.00396 & 0.53018 \\
\hline arrdwall.388.0 & 3.88 & 0.56517 & 0.00229 & 0.00458 & 0.56975 \\
\hline arrdwall.582.0 & 5.82 & 0.60455 & 0.00241 & 0.00482 & 0.60937 \\
\hline ardwall.873.0 & 8.73 & 0.63218 & 0.00275 & 0.0055 & 0.63768 \\
\hline arrdwall.136.o & 13.6 & 0.65324 & 0.00259 & 0.00518 & 0.65842 \\
\hline arrdwall.233.0 & 23.3 & 0.65294 & 0.00253 & 0.00506 & 0.658 \\
\hline arrdwall.471.0 & 47.1 & 0.59607 & 0.00218 & 0.00436 & 0.60043 \\
\hline arrdwall.524.0 & 52.4 & 0.58259 & 0.00241 & 0.00482 & 0.58741 \\
\hline ardwall.588.0 & 58.8 & 0.56608 & 0.00233 & 0.00466 & 0.57074 \\
\hline ardwall.773.0 & 77.3 & 0.52093 & 0.00186 & 0.00372 & 0.52465 \\
\hline arrdwall.110.0 & 110.6 & 0.45301 & 0.00178 & 0.00356 & 0.45657 \\
\hline arrdwall.188.0 & 188.2 & 0.34707 & 0.00141 & 0.00282 & 0.34989 \\
\hline
\end{tabular}


POEF-SH-30

Figure 4.8-2 An Array of 5.25" ID $\times 24^{n}$ Tall Cylinders of UF $4^{+011}$

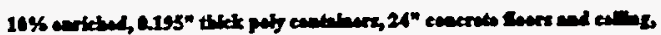

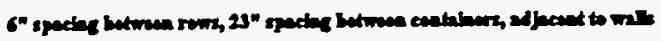

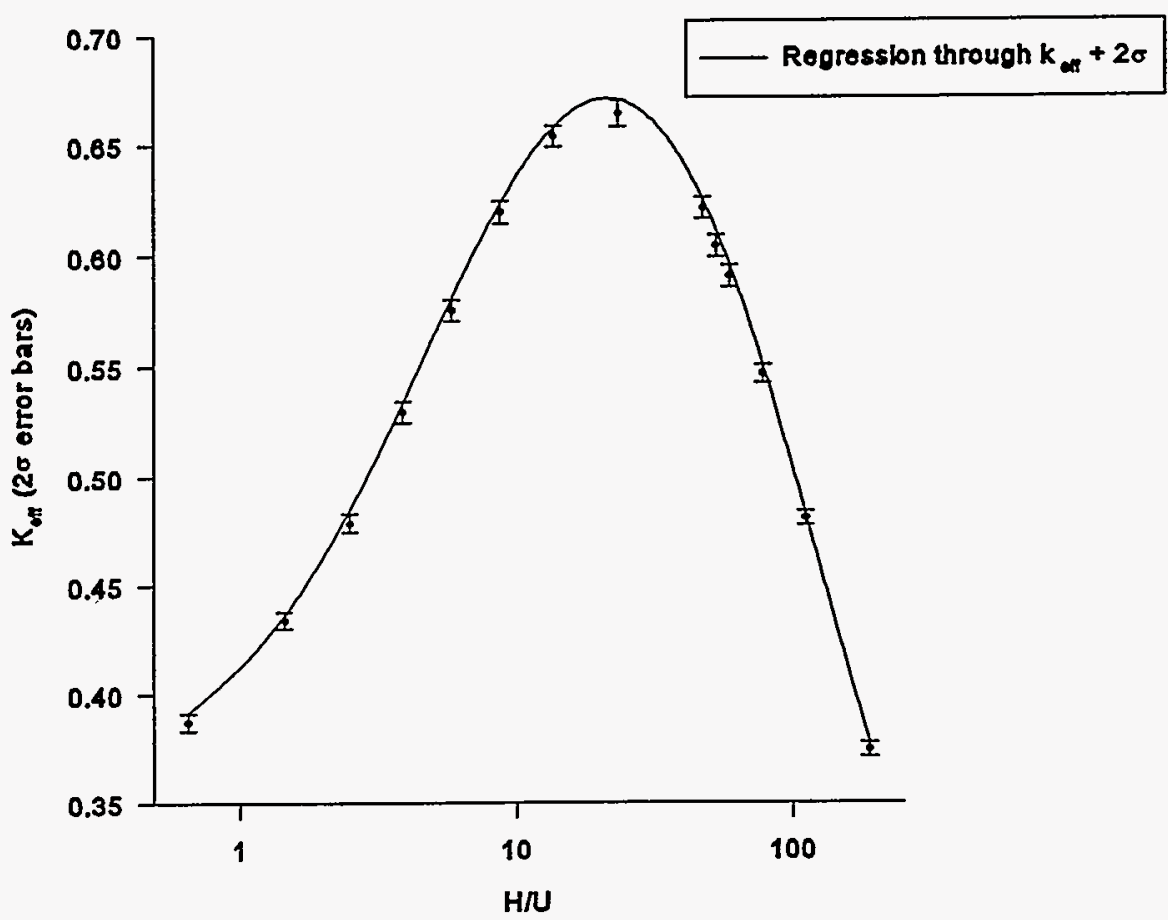

\begin{tabular}{|c|c|c|c|c|c|}
\hline \multicolumn{6}{|c|}{ An Array $5.25 \times 24^{\prime \prime}$ Cylinders of UF4+oil Next to a Wall } \\
\hline \multicolumn{6}{|c|}{$10 \%$ Enrichment, $0.195 "$ thick Poly Container } \\
\hline Case & $\mathrm{H} / \mathrm{U}$ & Keff & K_sig & $2 * K$ sig & Keff $+2^{\star}$ K_ sig \\
\hline arriwall.p65.0 & 0.65 & 0.3872 & 0.00197 & 0.00394 & 0.39114 \\
\hline arrfwall.145.0 & 1.45 & 0.43395 & 0.00194 & 0.00388 & 0.43783 \\
\hline arriwall.249.0 & 2.49 & 0.4785 & 0.00208 & 0.00416 & 0.48266 \\
\hline arrfwall.388.0 & 3.88 & 0.52936 & 0.00234 & 0.00468 & 0.53404 \\
\hline arriwall.582.0 & 5.82 & 0.57481 & 0.00237 & 0.00474 & 0.57955 \\
\hline arriwall.873.0 & 8.73 & 0.61912 & 0.00255 & 0.0051 & 0.62422 \\
\hline arrfwall.136.0 & 13.6 & 0.65311 & 0.00241 & 0.00482 & 0.65793 \\
\hline arrfwall.233.0 & 23.3 & 0.66334 & 0.00293 & 0.00586 & 0.6692 \\
\hline arriwall.471.0 & 47.1 & 0.6206 & 0.00242 & 0.00484 & 0.62544 \\
\hline arriwall.524.0 & 52.4 & 0.60363 & 0.00257 & 0.00514 & 0.60877 \\
\hline arriwall.588.0 & 58.8 & 0.58993 & 0.00249 & 0.00498 & 0.59491 \\
\hline arrfwall.773.o & 77.3 & 0.54611 & 0.00202 & 0.00404 & 0.55015 \\
\hline arriwall.110.0 & 110.6 & 0.48038 & 0.00173 & 0.00346 & 0.48384 \\
\hline arriwall.188.0 & 188.2 & 0.37425 & 0.0016 & 0.0032 & 0.37745 \\
\hline
\end{tabular}




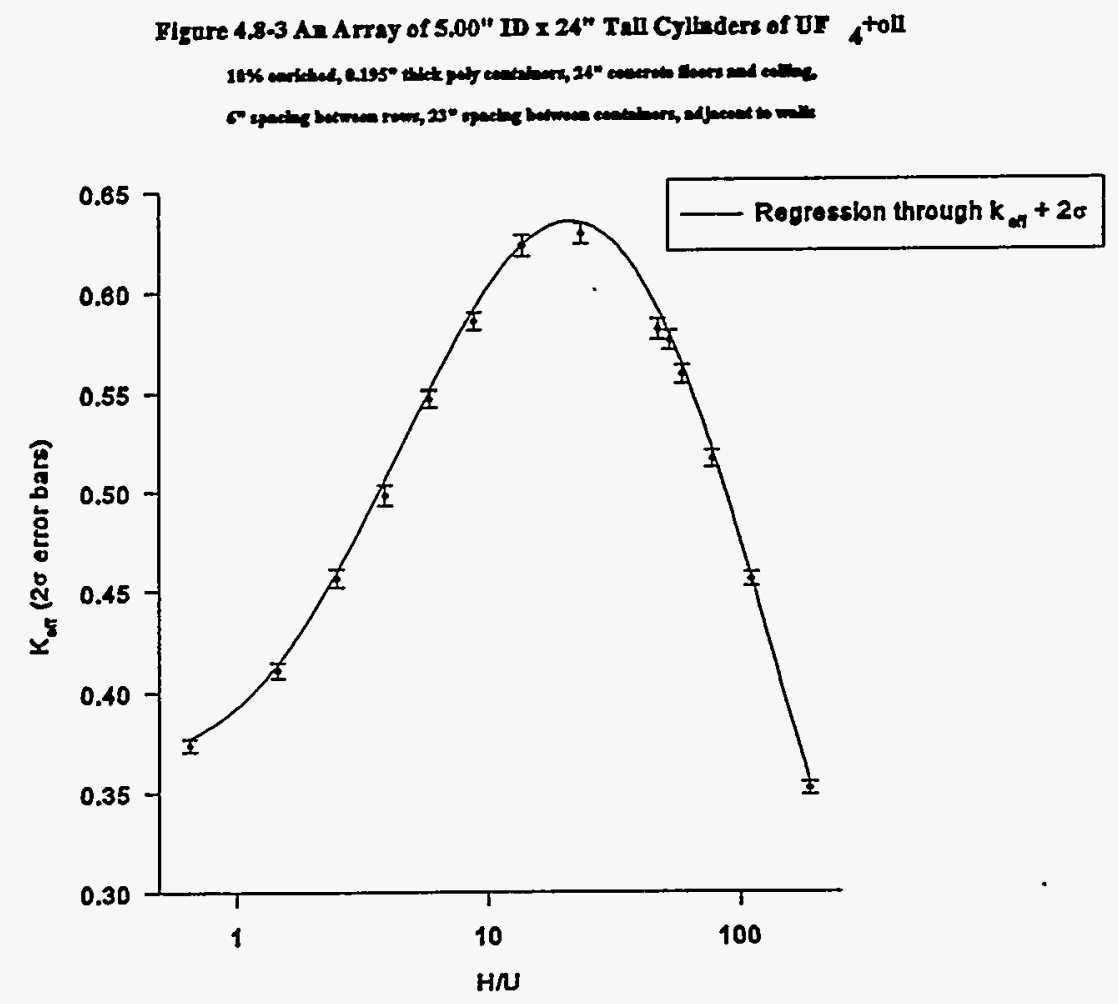

\begin{tabular}{|c|c|c|c|c|c|}
\hline \multicolumn{6}{|c|}{ An Array 5.00" $\times 24^{\prime \prime}$ Cylinders of UF4+oil Next to a Wall } \\
\hline \multicolumn{6}{|c|}{$10 \%$ Enrichment, $0.195^{\prime \prime}$ thick Poly Container } \\
\hline Case & $H / U$ & Keff & K s sig & $2 * \mathrm{~K}$ sig & Keff $+2^{\star} K$ sig \\
\hline arrewall.p65.0 & 0.65 & 0.37361 & 0.00159 & 0.00318 & 0.37679 \\
\hline arrewall.145.o & 1.45 & 0.41063 & 0.0019 & 0.0038 & 0.41443 \\
\hline arrewall.249.0 & 2.49 & 0.45636 & 0.00229 & 0.00458 & 0.46094 \\
\hline arrewall.388.0 & 3.88 & 0.49817 & 0.00261 & 0.00522 & 0.50339 \\
\hline arrewall.582.0 & 5.82 & 0.54685 & 0.00223 & 0.00446 & 0.55131 \\
\hline arrewall.873.o & 8.73 & 0.58513 & 0.00221 & 0.00442 & 0.58955 \\
\hline arrewall.136.0 & 13.6 & 0.62269 & 0.00269 & 0.00538 & 0.62807 \\
\hline arrewall.233.0 & 23.3 & 0.62854 & 0.00247 & 0.00494 & 0.63348 \\
\hline arrewall.471.o & 47.1 & 0.5809 & 0.00257 & 0.00514 & 0.58604 \\
\hline arrewall.524.0 & 52.4 & 0.57528 & 0.00239 & 0.00478 & 0.58006 \\
\hline arrewall.588.0 & 58.8 & 0.55855 & 0.00229 & 0.00458 & 0.56313 \\
\hline arrewall.773.o & 77.3 & 0.51596 & 0.00204 & 0.00408 & 0.52004 \\
\hline arrewall.110.0 & 110.6 & 0.45537 & 0.00175 & 0.0035 & 0.45887 \\
\hline arrewall.188.0 & 188.2 & 0.35183 & 0.00159 & 0.00318 & 0.35501 \\
\hline
\end{tabular}




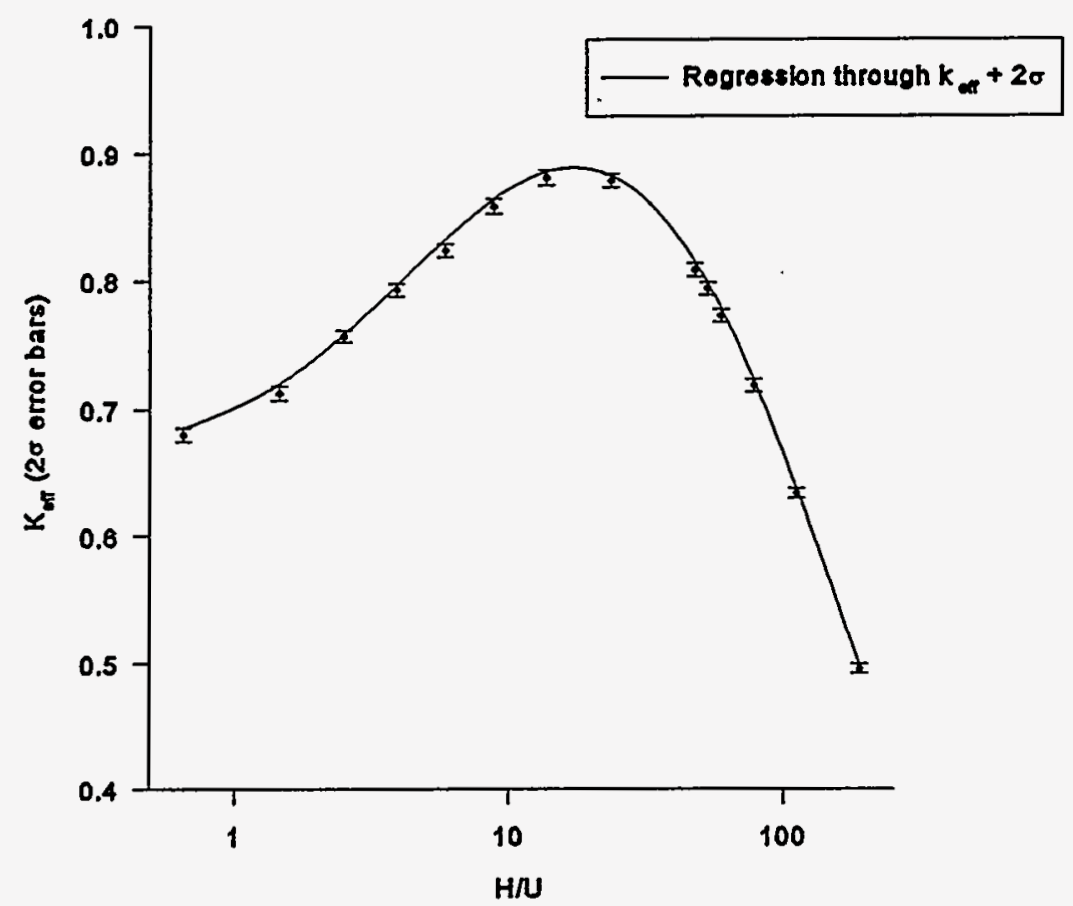

\begin{tabular}{|c|c|c|c|c|c|}
\hline \multicolumn{6}{|c|}{ Two Containers Side by Side Fully Reflected UF4+oil 10\% Enriched } \\
\hline Case & H/U & Keff & K sig & $2^{\star} \mathrm{K}$ sig & Keff+2* K_sig \\
\hline fc2-fr.p65 & 0.65 & 0.67985 & 0.0026 & 0.0052 & 0.68505 \\
\hline fc2-fr.145.o & 1.45 & 0.71256 & 0.00274 & 0.00548 & 0.71804 \\
\hline fc2-fr.249.0 & 2.49 & 0.7563 & 0.0024 & 0.0048 & 0.7611 \\
\hline fc2-fr.388.o & 3.88 & 0.79243 & 0.00243 & 0.00486 & 0.79729 \\
\hline fc2-fr.582.o & 5.82 & 0.82354 & 0.00272 & 0.00544 & 0.82898 \\
\hline fc2-fr.873.o & 8.73 & 0.85821 & 0.00273 & 0.00546 & 0.86367 \\
\hline fc2-fr.136.o & 13.6 & 0.88026 & 0.00288 & 0.00576 & 0.88602 \\
\hline fc2-fr.233.o & 23.3 & 0.87757 & 0.00267 & 0.00534 & 0.88291 \\
\hline fc2-fr.471.0 & 47.1 & 0.80784 & 0.0025 & 0.005 & 0.81284 \\
\hline fc2-fr.524.o & 52.4 & 0.7932 & 0.0024 & 0.0048 & 0.798 \\
\hline fc2-fr.588.0 & 58.8 & 0.77183 & 0.00243 & 0.00486 & 0.77669 \\
\hline fc2-fr.773.0 & 77.3 & 0.71813 & 0.00249 & 0.00498 & 0.72311 \\
\hline fc2-fr.110.0 & 110.6 & 0.633185 & 0.0021 & 0.0042 & 0.637385 \\
\hline fc2-fr.188.0 & 188.2 & 0.49561 & 0.00182 & 0.00364 & 0.49925 \\
\hline
\end{tabular}


Figure 4.8-5. An Array of 6.0" ID $\times 24^{\prime \prime}$ Tall Cylinders of UF ${ }_{4}+0$ ll

100\% Enslehed, 0.188" thlek poly containore, 24" concrete noor, 12" conerete walle and colling,

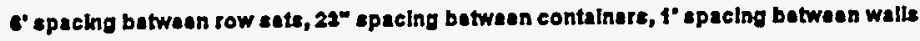

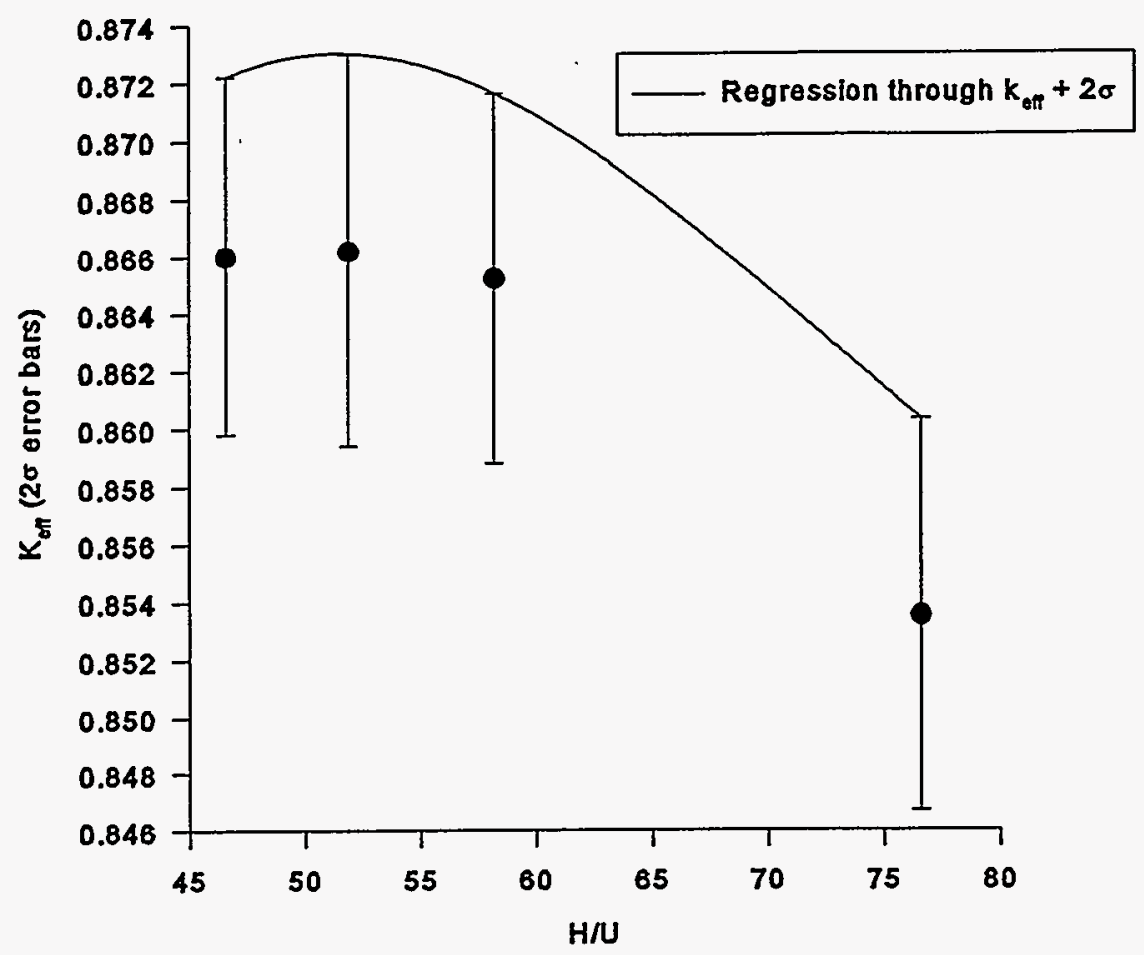

\begin{tabular}{|c|c|c|c|c|c|}
\hline \multicolumn{6}{|c|}{ An array of $5.0^{\prime \prime} \times 24$ " Tall Containers of UF4+oil $100 \%$ Enrichment } \\
\hline \multicolumn{6}{|c|}{ Spaced 1 ' from Wall } \\
\hline Case & $H / U$ & Keff & Siama & 2 Siama & Keff+2Sigma \\
\hline $\operatorname{arr12} 1.0$ & 46.6 & 0.866 & 0.0031 & 0.0062 & 0.8722 \\
\hline $\operatorname{arr12} 2.0$ & 51.9 & 0.8662 & 0.0034 & 0.0068 & 0.873 \\
\hline $\operatorname{arr12} 3.0$ & 58.2 & 0.8652 & 0.0032 & 0.0064 & 0.8716 \\
\hline $\operatorname{arr12} 4.0$ & 76.6 & 0.8535 & 0.0034 & 0.0068 & 0.8603 \\
\hline
\end{tabular}


Figure 4.8-6 An Array of 5.25" ID x 24" Tall Cylinder of UF ${ }_{4}$ and Oil Adjacent to Concrete Walls 10\% Enrichment, 24 ' coseroto floor, 12 " thick concteve wills, One overlouded storago portion

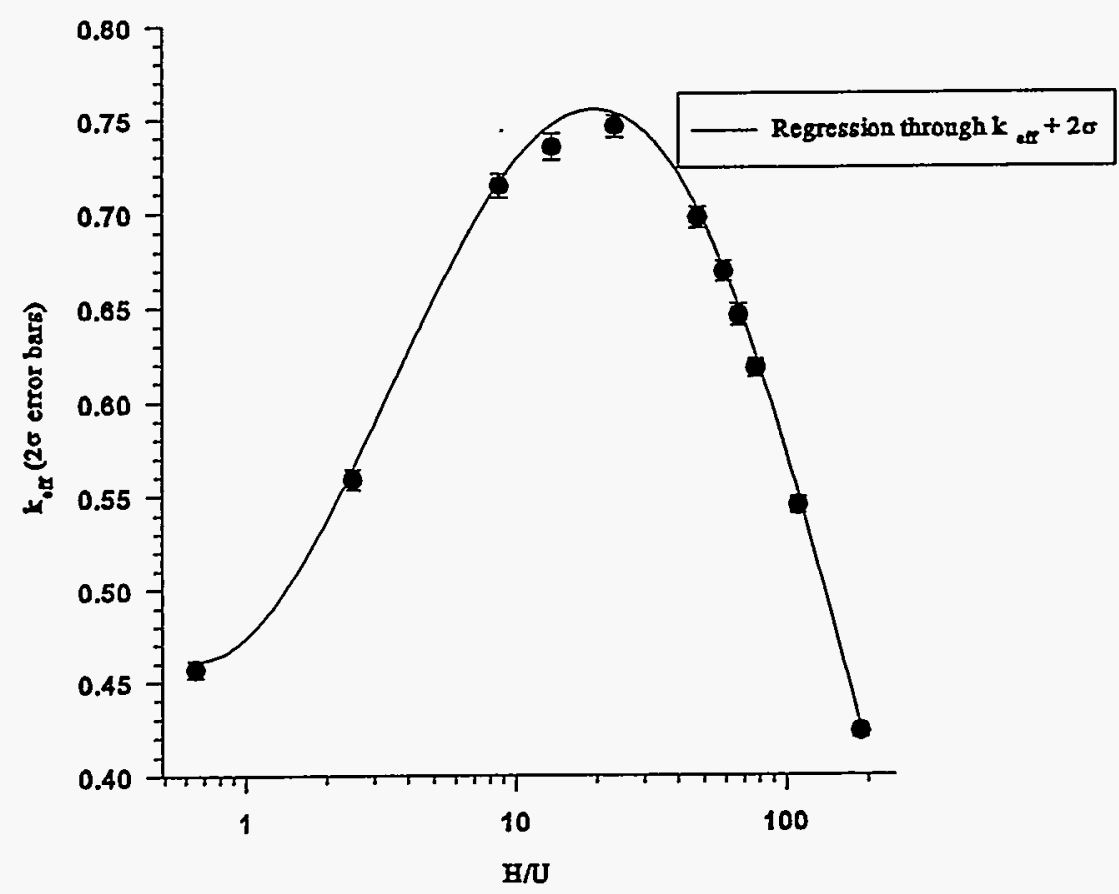

\begin{tabular}{|c|c|c|c|c|c|}
\hline \multicolumn{6}{|c|}{ 5.25" Cans, .0195" Poly Containers, Overloaded Storage Position } \\
\hline \multicolumn{6}{|c|}{ in an Array, Adjacent to Concrete Walls } \\
\hline $\mathrm{H} / \mathrm{U}$ & Case & Keff & K_sig & $2^{*} \operatorname{sig}$ & Keff+2sig \\
\hline 0.65 & $\operatorname{arrfen} 1.0$ & 0.4569 & 0.0023 & 0.0046 & 0.4615 \\
\hline 2.5 & $\operatorname{arrfen} 2.0$ & 0.5588 & 0.0027 & 0.0054 & 0.5642 \\
\hline 8.7 & $\operatorname{arrfcn} 3.0$ & 0.7138 & 0.0032 & 0.0064 & 0.7202 \\
\hline 13.6 & $\operatorname{arrfen} 4.0$ & 0.7344 & 0.0036 & 0.0072 & 0.7416 \\
\hline 23.3 & arrfen5.0 & 0.7455 & 0.003 & 0.006 & 0.7515 \\
\hline 47.1 & arrfen6.o & 0.6966 & 0.0028 & 0.0056 & 0.7022 \\
\hline 58.8 & $\operatorname{arrfcn} 7.0$ & 0.6687 & 0.0026 & 0.0052 & 0.6739 \\
\hline 66.9 & $\operatorname{arrfen} 8.0$ & 0.6452 & 0.0029 & 0.0058 & 0.651 \\
\hline 77.3 & arrfen9.0 & 0.6173 & 0.0025 & 0.005 & 0.6223 \\
\hline 110.6 & $\operatorname{arrfcn} 10.0$ & 0.5446 & 0.0021 & 0.0042 & 0.5488 \\
\hline 188.2 & $\operatorname{arrfcn} 11.0$ & 0.423 & 0.0016 & 0.0032 & 0.4262 \\
\hline
\end{tabular}




\subsection{Summary of Results}

Table 4.8-1 is a summary of the results for the $100 \%$ enrichment cases for each of the container types tested with $\mathrm{UO}_{2} \mathrm{~F}_{2}+\mathrm{H}_{2} \mathrm{O}, \mathrm{UF}_{4}+$ oil, and $\mathrm{U}_{3} \mathrm{O}_{8}$ for the various reflection models tested. The standard geometry models are described in Table 4.1-1. All 10\% enriched cases met the criteria $k_{\text {eff }}+2 \sigma \leq 0.95$ with the exception of one TACW case.

Table 4.9-1. Summary of Results for $100 \%$ Enriched Material

\begin{tabular}{|c|c|c|c|}
\hline Container Type/Material & Model & $\begin{array}{l}\text { Container Model } \\
\text { Description }\end{array}$ & $\begin{array}{l}k_{\text {eff }}+2 \sigma \leq \\
0.95\end{array}$ \\
\hline \multirow[t]{7}{*}{$4.32 "$ x 50.4" Poly/ $/ \mathrm{UO}_{2} \mathrm{~F}_{2}$} & SFR & Single, Full Reflection & Yes \\
\hline & SCC & Single, in Corner & Yes \\
\hline & TACW & Two, Adjacent to Wall & No \\
\hline & TSCW & Two, One Foot from Wall & Yes \\
\hline & $\mathrm{ACE}$ & Array, Standard & Yes \\
\hline & $\mathrm{AI}$ & Array, Interstitial Water & Yes \\
\hline & AOSP & $\begin{array}{l}\text { Array, Overloaded Storage } \\
\text { Position }\end{array}$ & Yes \\
\hline \multirow[t]{7}{*}{ 4.32" x 50.4" Poly/UF 4 +oil } & SFR & Single, Full Reflection & Yes \\
\hline & SCC & Single, in Corner & Yes \\
\hline & TACW & Two, Adjacent to Wall & No \\
\hline & $\mathrm{TSCW}$ & Two, One Foot from Wall & Yes \\
\hline & $\mathrm{ACE}$ & Array, Standard & Yes \\
\hline & $\mathrm{AI}$ & Array, Interstitial Water & Yes \\
\hline & AOSP & $\begin{array}{l}\text { Array, Overloaded Storage } \\
\text { Position }\end{array}$ & Yes \\
\hline
\end{tabular}


POEF-SH-30

\begin{tabular}{|c|c|c|c|}
\hline Container Type/Material & Model & $\begin{array}{c}\text { Container Model } \\
\text { Description }\end{array}$ & $\begin{array}{c}\mathrm{k}_{\mathrm{efr}}+2 \sigma s \\
0.95 \\
\end{array}$ \\
\hline \multirow[t]{7}{*}{$5.0^{\prime \prime} \times 24^{\prime \prime} \mathrm{Poly} / \mathrm{UO}_{2} \mathrm{~F}_{2}$} & SFR & $\begin{array}{l}\text { Single, Full Reflection - U } \\
\text { density limited to } 3.2 \mathrm{~g} / \mathrm{cc} \\
\text { for } H / U<4.0\end{array}$ & Yes \\
\hline & SCC & $\begin{array}{l}\text { Single, in Corner }-\mathrm{U} \\
\text { density limited to } 3.2 \mathrm{~g} / \mathrm{cc} \\
\text { for } \mathrm{H} / \mathrm{U}<4.0\end{array}$ & Yes \\
\hline & TACW & Two, Adjacent to Wall & No \\
\hline & TSCW & Two, One Foot from Wall & Yes \\
\hline & $\mathrm{ACE}$ & Array, Standard & Yes \\
\hline & $\mathrm{AI}$ & Array, Interstitial Water & Yes \\
\hline & AOSP & $\begin{array}{l}\text { Array, Overloaded Storage } \\
\text { Position }\end{array}$ & Yes \\
\hline \multirow[t]{6}{*}{ 5.0"x 24" Poly/ $\mathrm{UF}_{4}+$ oil } & SFR & $\begin{array}{l}\text { Single, Full Reflection - } \\
\text { Container Material Ignored }\end{array}$ & No \\
\hline & SCC & Single, in Corner & No \\
\hline & TACW. & Two, Adjacent to Wall & No \\
\hline & TSCW & Two, One Foot from Wall & No \\
\hline & $\mathrm{ACE}$ & Array, Standard & Yes \\
\hline & SMRC & $\begin{array}{l}\text { Single, More Reactive } \\
\text { Material }\end{array}$ & Yes \\
\hline \multirow[t]{3}{*}{$5.25^{\prime \prime} \times 24 " / \mathrm{UO}_{2} \mathrm{~F}_{2}$} & SFR & $\begin{array}{l}\text { Single, Full Reflection - } \\
\text { Container Material Ignored }\end{array}$ & No \\
\hline & SCC & Single, in Corner & No \\
\hline & TACW & Two, Adjacent to Wall & No \\
\hline $5.25^{\prime \prime} \times 24 " / \mathrm{UO}_{2} \mathrm{~F}_{2}$ & $\mathrm{TSCW}$ & Two, One Foot from Wall & No \\
\hline $5.25^{\prime \prime} \times 24 " / \mathrm{UF}_{4}+$ oil & SFR & $\begin{array}{l}\text { Single, Full Reflection - } \\
\text { Container Material Ignored }\end{array}$ & No \\
\hline
\end{tabular}


POEF-SH-30

\begin{tabular}{|c|c|c|c|}
\hline Container Type/Material & Model & $\begin{array}{l}\text { Container Model } \\
\text { Description }\end{array}$ & $\begin{array}{l}k_{e f r}+2 \sigma \leq \\
0.95\end{array}$ \\
\hline \multirow[t]{3}{*}{$5.25^{\prime \prime} \times 24 " / \mathrm{UF}_{4}$ +oil } & SCC & Single, in Corner & No \\
\hline & TACW & Two, Adjacent to Wall & No \\
\hline & TSCW & Two, One Foot from Wall & No \\
\hline \multirow[t]{2}{*}{$5.25^{\prime \prime} \times 15^{\prime \prime} / \mathrm{UO}_{2} \mathrm{~F}_{2}$} & SFR & $\begin{array}{l}\text { Single, Full Reflection - } U \\
\text { density to } 3.2 \mathrm{~g} / \mathrm{cc} \text { for } \\
\mathrm{H} / \mathrm{U}<4\end{array}$ & Yes \\
\hline & TSCW & $\begin{array}{l}\text { Two, One Foot from Wall - } \\
\mathrm{U} \text { density to } 3.2 \mathrm{~g} / \mathrm{cc} \text { for } \\
\mathrm{H} / \mathrm{U}<4\end{array}$ & Yes \\
\hline \multirow[t]{2}{*}{$5.25^{\prime \prime} \times 15^{\prime \prime} / \mathrm{UF}_{4}+$ oil } & SFR & Single, Full Reflection & No \\
\hline & SMRC & $\begin{array}{l}\text { Single, More Reactive } \\
\text { Material }\end{array}$ & Yes \\
\hline \multirow[t]{7}{*}{$5.25^{\prime \prime} \times 15^{\prime \prime} / \mathrm{U}_{3} \mathrm{O}_{8}$} & SFR & Single, Full Reflection & Yes \\
\hline & SCC & Single, in Corner & Yes \\
\hline & TACW & Two, Adjacent to Wall & No \\
\hline & TSCW & Two, One Foot from Wall & Yes \\
\hline & $\mathrm{ACE}$ & Array, Standard & Yes \\
\hline & $\mathrm{AI}$ & Array, Interstitial Water & Yes \\
\hline & AOSP & $\begin{array}{l}\text { Array, Overloaded Storage } \\
\text { Position }\end{array}$ & Yes \\
\hline \multirow[t]{4}{*}{$5.0^{\prime \prime} \times 91 / \mathrm{U}_{3} \mathrm{O}_{8}$} & SFR & Single, Full Reflection & Yes \\
\hline & SCC & Single, in Corner & Yes \\
\hline & TACW & Two, Adjacent to Wall & Yes \\
\hline & TSCW & Two, One Foot from Wall & Yes \\
\hline
\end{tabular}




\subsection{REFERENCES}

1.0 Plant Drawing DX-761-2340-M, "X-744G Polybottle," March 1990.

2.0 Plant Drawing BX-761-M373, “24" Polybottle,” November 1965.

3.0 Plant Drawing DX-761-2586-M, "Plastic Storage Container," June 1993.

4.0 Plant Drawing DX-761-M-981, “Container," September 1975.

5.0 Plant Drawing DX-761-2331-M, "5" x 15" Tin Container," March 1990.

6.0 Validation of the CSAS25 Calculation Sequence in Scale--4.2 and the 27 Energy Group ENDF/B-IV Cross Sections on the Battelle Process Safety and Risk Management IBM RS/6000 Workstation.

7.0 Conditional Validation of CSAS25 on Battelle Process Safety and Risk Management RS/6000 (Battelle 1994).

8.0 Configuration Control Plan for Process Safety and Risk Management Nuclear Safety Software \& Hardware Systems (IBM/RS6000) (Battelle, August 1994).

9.0 Personal Correspondence, R. Tayloe to D. D'Aquila, June 2, 1995, “Standards for Analysis of Small Containers at the Portsmouth Plant," 1995.

$10.0 \mathrm{~K} / \mathrm{ETO}-143$, Barber E.J. "Reaction of Uranium Hexafluoride with Hydrocarbon Oil," Enrichment Technical Support Department, August 1994.

11.0 KY/L-1699, Russell R.G. "Investigation of Hygroscopic Properties of Uranyl Fluoride," Paducah Gaseous Diffusion Plant, November 1994.

12.0 POEF-T-3545, Tayloe, R.W. "Calculating Atomic Number Densities For Uranium Compounds," Battelle Memorial Institute, January 1993.

13.0 LA-1086-MS, Paxton, H.C. and Pruvost, N.L. "Criticality Dimensions of Systems Containing U235, Pu-239, and U-233 - 1986 Revision," Los Alamos National Laboratory, July 1987.

14.0 Perry, Robert H. and Cecil H. Chilton, Chemical Engineers' Handbook, 5th ed., McGraw-Hill Book Co.

15.0 SCALE 4.1 Manual, March 1992. 
POEF-SH-30

\section{REFERENCES (Continued)}

16.0 ANSI/ANS - 8.1, American National Standard for Nuclear Criticality Safety in Operations with Fissionable Materials Outside Reactors, American Nuclear Society, October 1983.

17.0 GAT - 225, Rev. 4, Nuclear Criticality Safety Guide for the Portsmouth Gaseous Diffusion Plant.

18.0 Benedict M., Pigford T., Levi H. W. "Nuclear Chemical Engineering 2nd." MHBC, 1989. 


\subsection{APPENDIX}

KENO Geometry Data File for Single Fully Reflected Container (SFR)

$\mathrm{H} 2 \mathrm{O}+2 \mathrm{END}$

REG-CONCRETE 3 END

POLY(H2O) 4 END

END COMP

FULLY REFLECTED 4.32" ID x 50" TALL CYLINDER

' 4.32" ID x 50" TALL CYLINDER OF MATERIAL \#1 (FISSILE),

' 0.1 " THICK CONTAINER (MATERIAL \#4) ON ALL SIDES OF FISSILE MATERIAL,

' 12" (MINIMUM) WATER REFLECTOR ON TOP \& SIDES, 24" CONCRETE PAD

READ PARAM NUB=YES FDN=YES END PARAM

READ GEOM

ZCYLINDER $115.4864 \quad 127.00 \quad 0.0$

ZCYLINDER $415.7404 \quad 127.254-0.254$

CUBOID 214 P $36.2204 \quad 157.734-0.254$

CUBOID 314 4P36.2204 $157.734-61.214$

END GEOM

END DATA

END 
KENO Geometry Data File for a Single Container in a Corner (SCC)

H2O 2 END

REG-CONCRETE 3 END

END COMP

4.32" ID x 50" TALL CYLINDER IN A CORNER

' 4.32" ID x 50" TALL CYLINDER OF MATERIAL \#1 (FISSILE), 24" CONCRETE PAD,

' 12" CONCRETE WALLS AND CEILING, $100 \mathrm{~kg}$ L-SHAPED WATER REFLECTOR

' SIMULATING A PERSON, NO CONTAINER MODELED

READ PARAM NUB=YES FDN=YES END PARAM

READ GEOM

ZCYLINDER $115.49 \quad 127.00 \quad 0.0$

CUBOID 014 4P5.49 $127.00 \quad 0.0$

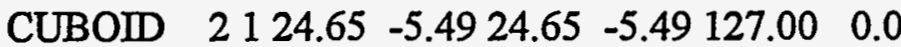

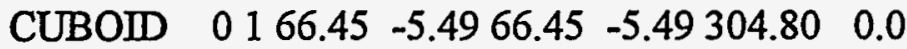

CUBOID $3166.45-35.9766 .45-35.97335 .28-60.96$

END GEOM

END DATA

END 
KENO Geometry Data File for Two Cylinders Adjacent to a Wall (TACW)

$\mathrm{H} 2 \mathrm{O} 2 \mathrm{END}$

REG-CONCRETE 3 END

END COMP

TWO 4.32" ID x 50" TALL CYLINDERS NEXT TO A WALL

' TWO 4.32" ID x 50" TALL CYLINDERS OF MATERIAL \#1 (FISSILE),

' 24" CONCRETE PAD, 12" CONCRETE WALLS AND CEILING, $100 \mathrm{~kg}$ WATER

' REFLECTOR SIMULATING A PERSON (18" wide x 7" deep x 50" tall)

READ PARAM NUB=YES FDN=YES END PARAM

READ GEOM

UNIT 1

ZCYLINDER $115.49 \quad 127.00 \quad 0.0$

CUBOID $0114 \mathrm{P} 5.49127 .00 \quad 0.0$

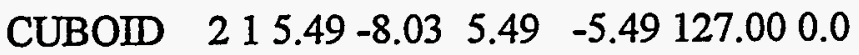

CUBOID $\quad 015.49-17.375 .49 \quad-5.49127 .000 .0$

CUBOID $215.49-17.3723 .27-5.49127 .000 .0$

CUBOID $015.49-85.9566 .45-5.49304 .800 .0$

CUBOID $315.49-85.9566 .45-35.97335 .28$-60.96

UNIT 2

ZCYLINDER $115.49 \quad 127.00 \quad 0.0$

CUBOID $014 \mathrm{P} 5.49127 .00 \quad 0.0$

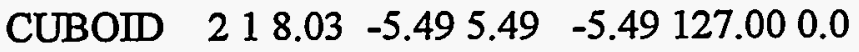

CUBOID $\quad 0 \quad 1 \quad 17.37-5.495 .49 \quad-5.49127 .000 .0$

CUBOID $2117.37-5.4923 .27 \quad-5.49127 .000 .0$

CUBOID $0185.95-5.4966 .45-5.49304 .800 .0$

CUBOID $3185.95-5.4966 .45-35.97335 .28-60.96$

END GEOM

READ ARRAY NUX=2 NUY=1 NUZ=1 FILL 12 END FILL END ARRAY END DATA

END 
KENO Geometry Data File for Two Cylinders One Foot from a Wall (TSCW)

$\mathrm{H} 2 \mathrm{O} 2 \mathrm{END}$

REG-CONCRETE 3 END

POLY(H2O) 4 END

END COMP

TWO 4.32" ID x 50" TALL CYLINDERS 12" FROM A WALL

' TWO 4.32" ID x 50" TALL CYLINDERS OF MATERIAL \#1 (FISSILE),

' 0.195" THICK CONTAINER (MATERIAL \#4) AROUND EACH CYLINDER OF FISSILE

' MATERIAL, 24" CONCRETE PAD, 12" CONCRETE WALLS AND CEILING, 100 kg

' WATER REFLECTOR SIMULATING A PERSON (18" wide x 7" deep x 50" tall)

READ PARAM NUB=YES FDN=YES NPG=600 END PARAM

READ GEOM

UNIT 1

ZCYLINDER $415.49 \quad 0.50 \quad 0.0$

ZCYLINDER $115.49 \quad 127.50 \quad 0.0$

ZCYLINDER $415.99 \quad 128.00 \quad 0.0$

CUBOID 014 4P5.99 $128.00 \quad 0.0$

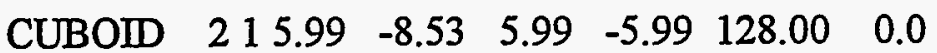

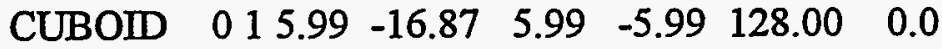

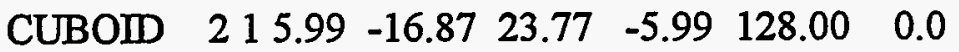

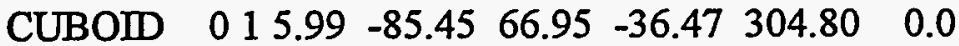

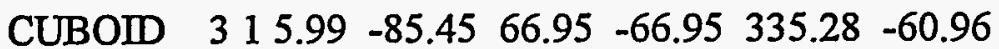

UNIT 2

ZCYLINDER $415.49 \quad 0.50 \quad 0.0$

ZCYLINDER $115.49 \quad 127.50 \quad 0.0$

ZCYLINDER $415.99 \quad 128.00 \quad 0.0$

CUBOID 014 4P5.99 $128.00 \quad 0.0$

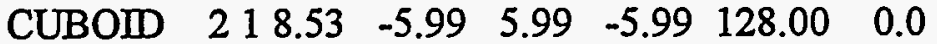

$\begin{array}{lllllllll}\text { CUBOID } & 0 & 1 & 16.87 & -5.99 & 5.99 & -5.99 & 128.00 & 0.0\end{array}$

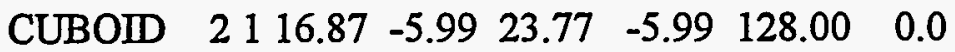

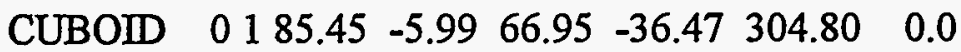

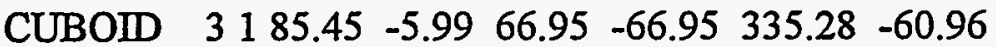

END GEOM

READ ARRAY NUX=2 NUY=1 NUZ=1 FILL 12 END FILL END ARRAY

END DATA

END 
KENO Geometry Data File for a Standard Array of Containers (ACE)

$\mathrm{H} 2 \mathrm{O} 20.001 \mathrm{END}$

REG-CONCRETE 3 END

POLY(H2O) 4 END

END COMP

ARRAY OF CONTAINERS WITH FISSILE MATERIAL \#1

' 4.32" ID x 50" TALL CYLINDERS OF MATERIAL \#1 (FISSILE),

' 0.195 " THICK CONTAINER (MATERIAL \#4) AROUND EACH CYLINDER OF FISSILE

' MATERIAL, 24" CONCRETE PAD, 12" CONCRETE WALLS AND CEILING

READ PARAM NPG=600 GEN=153 MKP=YES MKU=YES

MKA=YES NUB=YES FDN=YES END PARAM

READ GEOM

' UNIT 1 IS A 23" $\times 23 " x 10$ ' TALL CUBOID CONTAINING THE FISSILE CYLINDER

UNIT 1

ZCYLINDER $415.49 \quad 0.500 .0$

ZCYLINDER $1115.49127 .50 \quad 0.0$

ZCYLINDER $4115.99 \quad 128.00 \quad 0.0$

CUBOID $\quad 21 \quad 4$ P $29.21304 .80 \quad 0.0$

' UNIT 2 IS A CUBOID NEEDED TO PUT 6 ' OF SPACE BETWEEN THE ROW SETS

UNIT 2

CUBOID $2 \begin{array}{lllllll}2 & 58.42 & 0.0 & 124.46 & 0.0 & 304.80 & 0.0\end{array}$

' UNIT 3 IS A CUBOID NEEDED TO PUT 3' OF SPACE BETWEEN THE TOP ROW

' AND THE MIRROR ALBEDO ON THE +Y BOUNDARY

UNIT 3

$\begin{array}{lllllllll}\text { CUBOID } & 2 & 1 & 58.42 & 0.0 & 62.23 & 0.0 & 304.80 & 0.0\end{array}$

' GLOBAL UNIT 8 IS AN ARRAY OF FISSILE UNITS AND SPACING UNITS

' SURROUNDED BY A CUBOID OF SPACE TO SET THE DISTANCE FROM THE WALLS

' TO THE NEAREST CONTAINER, AND A CUBOID OF REG-CONCRETE TO MAKE UP

' THE WALLS, CEILING, AND FLOOR

GLOBAL UNIT 8

ARRAY $1 \quad 0.0 \quad 0.0 \quad 0.0$

CUBOID $2111168.4 \quad-31.75 \quad 420.37 \quad-31.75 \quad 304.80 \quad 0.0$

$\begin{array}{llllllll}\text { CUBOID } 3 & 1 & 1168.4 & -62.23 & 420.37 & -62.23 & 335.28 & -60.96\end{array}$

END GEOM

READ ARRAY ARA $=1$ NUX $=20$ NUY $=6$ NUZ=1 FILL

11111111111111111111

11111111111111111111

22222222222222222222

11111111111111111111

11111111111111111111

33333333333333333333

END FILL END ARRAY

READ BOUNDS +XB=MIRROR +YB=MIRROR END BOUNDS

END DATA 
KENO Geometry Data File for a Contingency Case for a Standard Array of Containers (AOSP)

$\mathrm{H} 2 \mathrm{O} 2 \mathrm{END}$

REG-CONCRETE 3 END

POLY(H2O) 4 END

END COMP

CONTINGENCY CASE FOR ARRAY OF CONTAINERS WITH FISSILE MATERIAL \#1

' 4.32" ID x 50" TALL CYLINDERS OF MATERIAL \#1 (FISSILE),

' 0.195" THICK CONTAINER (MATERIAL \#4) AROUND EACH CYLINDER OF FISSILE

' MATERIAL, 24" CONCRETE PAD, 12" CONCRETE WALLS AND CEILING

READ PARAM NPG=600 GEN=153 MKP=YES MKU=YES

MKA=YES NUB=YES FDN=YES END PARAM

READ GEOM

' UNIT 1 IS A 23"x23"x10' TALL CUBOID CONTAINING THE FISSILE CYLINDER

UNIT 1

ZCYLINDER $4 \quad 1 \quad 5.49 \quad 0.50 \quad 0.0$

$\begin{array}{llllllll}\text { ZCYLINDER } & 1 & 1 & 5.49 & 127.50 & 0.0\end{array}$

ZCYLINDER $\begin{array}{llllll}4 & 1 & 5.99 & 128.00 & 0.0\end{array}$

$\begin{array}{llllllll}\text { CUBOID } & 0 & 1 & 4 \mathrm{P} 29.21 & 304.80 & 0.0\end{array}$

' UNIT 2 IS A CUBOID NEEDED TO PUT 6' OF SPACE BETWEEN THE ROW SETS

UNIT 2

$\begin{array}{llllllllll}\text { CUBOID } & 0 & 1 & 58.42 & 0.0 & 124.46 & 0.0 & 304.80 & 0.0\end{array}$

' UNIT 3 IS A CUBOID NEEDED TO PUT 3' OF SPACE BETWEEN THE TOP ROW

' AND THE MIRROR ALBEDO ON THE +Y BOUNDARY

UNIT 3

$\begin{array}{llllllllll}\text { CUBOID } & 0 & 1 & 58.42 & 0.0 & 62.23 & 0.0 & 304.80 & 0.0\end{array}$

' UNIT 4 IS A 23 " $\times 23 " x 10$ ' TALL CUBOID CONTAINING THE FISSILE CYLINDER

' AND CONTINGENCY FISSILE CYLINDER. THIS UNIT IS PLACED IN THE CORNER

' OF THE ARRAY CLOSEST TO THE TWO WALLS

UNIT 4

ZCYLINDER $4 \quad 1 \quad 5.49 \quad 0.50 \quad 0.0$

ZCYLINDER $1115.49 \quad 127.50 \quad 0.0$

ZCYLINDER $4 \begin{array}{lllll}1 & 5.99 & 128.00 & 0.0\end{array}$

$\begin{array}{lllllllllll}\text { CUBOID } & 0 & 1 & 29.21 & -22.03 & 29.21 & -22.03 & 128.00 & 0.0\end{array}$

$\begin{array}{llllll}\text { HOLE } & 5 & 8.48 & 8.48 & 0.0\end{array}$

$\begin{array}{llllllll}\text { CUBOID } & 2 & 1 & 4 \mathrm{P} 29.21 & 128.00 & 0.0\end{array}$

$\begin{array}{llllllllll}\text { CUBOID } & 0 & 1 & 4 \mathrm{P} 29.21 & 304.80 & 0.0\end{array}$

UNIT 5

ZCYLINDER $4 \quad 1 \quad 5.49 \quad 0.50 \quad 0.0$

ZCYLINDER $1115.49 \quad 127.50 \quad 0.0$

ZCYLINDER $4 \quad 1 \quad 5.99128 .00 \quad 0.0$

' GLOBAL UNIT 8 IS AN ARRAY OF FISSILE UNITS AND SPACING UNITS

' SURROUNDED BY A CUBOID OF SPACE TO SET THE DISTANCE FROM THE WALLS

' TO THE NEAREST CONTAINER, AND A CUBOID OF REG-CONCRETE TO MAKE UP 
' THE WALLS, CEILING, AND FLOOR

GLOBAL UNIT 8

$\begin{array}{llll}\text { ARRAY } 1 & 0.0 & 0.0 & 0.0\end{array}$

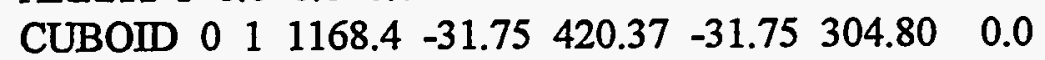

$\begin{array}{lllllllll}\text { CUBOID } 3 & 1 & 1168.4 & -62.23 & 420.37 & -62.23 & 335.28 & -60.96\end{array}$

END GEOM

READ ARRAY ARA $=1$ NUX $=20$ NUY $=6$ NUZ=1 FILL

41111111111111111111

11111111111111111111

22222222222222222222

11111111111111111111

11111111111111111111

33333333333333333333

END FILL END ARRAY

READ BOUNDS +XB=MIRROR +YB=MIRROR END BOUNDS

END DATA

END 